\title{
Neutron Radiation, Radiation Measurement and the JLab APEX Experiment
}

\author{
Maduka Manaram Kaluarachchi \\ Colombo, Sri Lanka \\ B.Sc., University of Colombo, Sri Lanka, 2009

\begin{abstract}
A Dissertation presented to the Graduate Faculty
of the University of Virginia in Candidacy for the Degree of

Doctor of Philosophy
\end{abstract} \\ Department of Physics

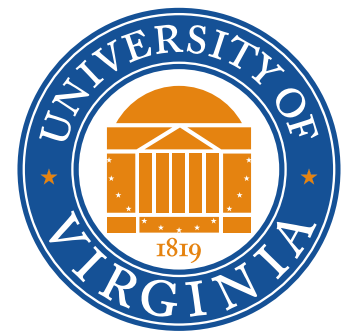 \\ University of Virginia \\ August, 2017
}




\begin{abstract}
The $\mathrm{A}^{\prime}$ Experiment (APEX), which is approved to run at the Thomas Jefferson Accelerator Facility (JLab) Hall A, will search for a new vector boson that is hypothesized to be a possible force carrier that couples to dark matter. APEX results should be sensitive to the mass range of $65 \mathrm{MeV}$ to $550 \mathrm{MeV}$, and high sensitivity will be achieved by means of a high intensity $\sim 100 \mu \mathrm{A}$ beam on a $\sim 0.3 \mathrm{~g} / \mathrm{cm}^{2}$ Tungsten target resulting in very high luminosity. The experiment should be able to observe an $A^{\prime}$ with a coupling constant $\alpha^{\prime} \sim 1 \times 10^{7}$ times smaller than the electromagnetic coupling constant $\alpha$.

To deal safely with such enormous intensity and luminosity, a full radiation analysis must be used. A Geant4 Monte Carlo simulation was performed to study the radiation environments expected in the JLab Hall A during the APEX experiment. We benchmarked our simulations with measurements carried out during past experiments such as the ${ }^{208} \mathrm{~Pb}$ Radius Experiment (PREX-I). Our PREX-I simulations agreed reasonably well with measurements performed by measuring the change in dark current in a Silicon Photomultipliers as a means of radiation damage. However, our simulations were initially significantly higher than the neutron dose measurements (using NP100) in Hall A. The apparent disagreement hints to several potential problems in NP100 measurements including the dead-time. Further, we identified radiation sources in the beam line and tested several shielding strategies to lower the radiation in Hall A. We characterized several thermal neutron detectors and also studied possibility of damage to electronics due to neutron radiation. We successfully demonstrated that the APEX experiment can be carried out in JLab Hall A without worrying about radiation damage to expensive electronics.
\end{abstract}

The $A^{\prime}$ will appear as a narrow resonance in the invariant mass spectrum of $e^{+} e^{-}$ 
pairs resulting from the electron beam scattering off the APEX target. In this search for the $A^{\prime}$, it is critical to have a good mass resolution which is mainly dominated by the track measurement uncertainties. We studied a novel method to calibrate the HRS optics which will improve the accuracy of track measurements. Our results will be used to design HRS calibration runs for the APEX experiment. 


\section{Acknowledgements}

First and foremost I want to thank my advisor Prof. Gordon Cates. It has been an honor to work with him all these years and learn from one of the best in the field. I am very fascinated by his excitement and enthusiasm when he talks about physics and it always kept me motivated, even during tough times in the Ph.D. pursuit. Apart from physics, I really enjoyed our discussions on politics, religion, culture, music and his life experiences. Prof. Cates supported me not only by providing a research assistantship for over five years, but also academically and emotionally through the rough road to finish this thesis. Above all, I am thankful for being such a nice person, which made my life as a graduate student joyful.

The members of my research and thesis committee, Prof. Dinko Počanić, Prof. Don Crabb and Prof. Wilson Miller provided feedback through out these years to steer my project and thesis to a success .

I would like to thank Dr. Bogdan Wojtsekhowski at Jefferson Lab for the guidance and advice throughout my project. Dr. Wojtsekhowski's work ethics and discipline were good lessons for me to become a good scientist. I would also like to thank Prof. Kent Paschke, Dr. Sergey Abrahamyan, Dr. Seamus Riordan, Dr. Pavel Degtiarenko and Dr. Vashek Vylet for supporting me and providing me with resources to carry out the projects.

I am very grateful to Prof. Nilanga Liyanage for all the advice through out these years. Dr. Liyanage is one of the role models I look up to in my career. I consider myself very lucky to have the opportunity to be one of the Sri Lankan students Dr. Liyanage recruited for the UVa Physics. I also really appreciate Prof. Krishni Wijesooriya for her advice and support.

I would like to extend my gratitude to Dr. Vladimir Nelyubin for teaching me 
about optics and Monte Carlo simulations, few of many of his expertise. Dr. William A. Tobias always took time to teach me about the gas system and the importance of taking notes. There is no argument that Vladimir and Al are the two most valuable resources in Cates Lab. I loved to chat with these two, where we always ended up with a good laugh. I would also like to thank Dr. Bryan Wright for all the computer support.

My two lab buddies, Yunxiao Wang and Dan Matys always were there for me. We enjoyed mocking each other and shared good stories. We enjoyed having coffee at Castle for a break and eating "young chicken" for dinner at Taste of China. I learned a lot from them, most of all I enjoyed hanging around with them to relieve and share the graduate school stress.

I am also grateful to all my peers at UVa physics for providing support, especially, Mikhail, Abha, Dustin and Darshana. We have a close knit family like bond with all the Sri Lankan friends in Charlottesville who never let us feel alone in a foreign country.

I must express my gratitude to my loving wife, Kanchana, for her continued support, encouragement and especially her willingness to proof read countless pages of meaningless physics equations. Last, but not least, I would like to thank my loving parents for their constant love and support. 


\section{Contents}

$\begin{array}{ll}\text { Abstract } & \text { ii }\end{array}$

Acknowledgements $\quad$ iv

List of Tables $\quad$ x

List of Figures $\quad$ xiii

1 Introduction 1

1.1 The challenge of high radiation experiments . . . . . . . . . . 2

1.2 The physics of APEX . . . . . . . . . . . . . 3

1.2.1 Motivation for the JLab APEX experiment . . . . . . . . . . . 4

1.2.2 The $A^{\prime}$ boson as a probe of dark matter . . . . . . . . . 5

1.2.3 $A^{\prime}$ production in fixed target experiments . . . . . . . . 8

1.3 APEX test run . . . . . . . . . . . . . . . . . 12

1.4 Brief overview of the approved JLab APEX experiment . . . . . . . 13

1.5 Structure of this thesis . . . . . . . . . . . . . . . . . . . 14

2 The APEX Experiment 17

2.1 APEX target . . . . . . . . . . . . . . . 17

2.2 APEX Septum Magnet . . . . . . . . . . . . . . . . 21 
2.3 The detector package . . . . . . . . . . . . . . . . 22

2.4 Background Rates . . . . . . . . . . . . . . . . . . 25

2.5 Particle identification . . . . . . . . . . . . . . . 27

2.6 Measurement of the particle track . . . . . . . . . . . . . . 30

2.7 Trigger and DAQ . . . . . . . . . . . . . . 33

\section{Monte-Carlo simulation of radiation environments during APEX 37}

3.1 Geant4 configuration and a consistency check with previous codes . . 38

3.2 Geant4 model . . . . . . . . . . . . . . . . . . . . . 42

3.2.1 Hall A . . . . . . . . . . . . . . . . . . . 42

3.2.2 Hall A Beamline . . . . . . . . . . . . . . . . 46

3.2.3 Components in the High Resolution Spectrometer (HRS) . . . 46

3.2.4 APEX experimental setup and beamline components . . . . 48

3.2.5 PREX-I experimental setup and beamline components . . . . 50

3.2.6 Real Compton Scattering (RCS) experimental setup and beamline components .................. 57

3.3 Relative comparisons of APEX with other experiments . . . . . . 58

3.3.1 Particle fluence spectra . . . . . . . . . . . . . . . 59

3.3.2 Radiation damage to semiconductor electronics . . . . . . . . 61

3.3.3 Comparison of APEX radiation levels . . . . . . . . . . . 63

3.4 Absolute comparisons of our Geant4 simulations . . . . . . . . . . 76

3.4.1 Comparison with silicon damage measurements . . . . . . . 76

3.4.2 Comparison with neutron dose rate measurements . . . . . . . 79

3.4.3 Summary of absolute comparisons . . . . . . . . . . . . . . 89

3.5 Radiation sources and possible improvements in shielding . . . . . . . 93

3.6 A summary of Monte-Carlo simulation of radiation during APEX . . 111 
4 Scintillating Fiber Detector (SciFi) 115

4.1 Mass resolution . . . . . . . . . . . . . . . . . . 115

4.2 Optics calibration using sieve slit method . . . . . . . . . . . . . 117

4.3 Optics calibration using an active scintillation fiber (SciFi) detector . 122

4.4 SciFi detector . . . . . . . . . . . . . . . . . . . 122

4.5 Experiment Setup to evaluate efficiency of the SciFi detector . . . . . 127

4.6 Statistical model and fitting . . . . . . . . . . . . . 128

4.6.1 Evaluate the $\mu$ based on path length through a fiber . . . . . . 133

4.6.2 Evaluate $\mu$ based on true detector response . . . . . . . . . . . 140

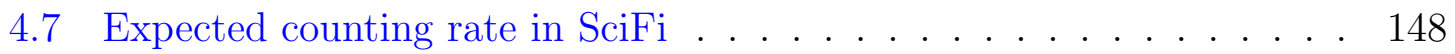

5 Evaluation of neutron scattering effects in a JLab radiation-detector calibration facility using Bonner spheres 153

5.1 Bonner sphere and its operation . . . . . . . . . . . . . . 154

5.2 A Bonner sphere Spectrometer at JLab . . . . . . . . . . . . . . . . 162

5.3 Calibration of the BSS with an ${ }^{241} \mathrm{Am}$ - Be source . . . . . . . . . . . 164

5.3.1 Calibration facility at JLab . . . . . . . . . . . . . 165

5.3.2 Evaluate scatter-free count rates in Bonner spheres . . . . . . 168

5.3.3 Evaluate scatter-free count rates using simulations . . . . . . . 173

5.4 Monte-carlo simulation of neutron scattering . . . . . . . . . . 175

5.5 Analyzing scattered neutron spectra . . . . . . . . . . . . 184

5.6 Summary of evaluating neutron scattering effects using Bonner spheres 187

$\begin{array}{lll}6 & \text { Summary } & 188\end{array}$

A Geant4 simulations of the response function of the neutron detector SNOOPY 
B Maximum-likelihood expectation-maximization (MLEM) unfolding210 


\section{List of Tables}

2.1 Run plan for production targets. Total target thickness is given as a percentage of radiation length for each target material. . . . . . . .

2.2 Main design characteristics of the Hall A High Resolution Spectrometers at a nominal target position. The resolution values are for the

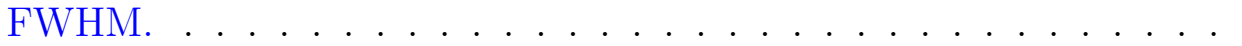

2.3 Expected counting rates for APEX experiment. Settings A and B comprise the primary run plan, while setings $\mathrm{C}$ and $\mathrm{D}$ are additional settings at intermediate energies. . . . . . . . . . . . .

3.1 Comparison of simulated particle fluence of PREX-I experiment using a Geant4 model developed by the PREX collaboration and the model developed for this work. The area-method, described in the text, was used to calculate the particle fluence in both cases. . . . . . . . .

3.2 Inner diameter, outer diameter and length of different sections of the downstream beam line are listed here. . . . . . . . . . . . .

3.3 This table summarizes the calculation of $1 \mathrm{MeV}$ neutron equivalent fluence at HRS control electronics for PREX-I, RCS and APEX experiments. . . . . . . . . . . . . . . . . 
3.4 Table summarizes the calculation of $1 \mathrm{MeV}$ neutron equivalent fluence at different locations in the hall A during APEX experiment. The second column is the fluence only from neutrons and the thrid column is the fluence only from $e^{+} / e^{-}$. All the proposed APEX settings are included in this calculation. . . . . . . . . . . . . . . . . 74

3.5 Summary of measured and predicted SNOOPY readings in JLab Hall A for the PREX-I and RCS experiments. All the measurements and calculations are assuming a $100 \mu \mathrm{A}$ beam current. . . . . . . . . .

3.6 Calculated equivalent $1 \mathrm{MeV}$ neutron fluence at the HRS electronics area and in the upstream of the JLab Hall A. Only the APEX 3.3 GeV and the APEX $4.4 \mathrm{GeV}$ settings were considered. The column "With Collimator" indicates the neutron fluence when a tungsten collimator with a wall thickness of $1.5 \mathrm{~cm}$ was used within the septum magnet. . 112

4.1 Expected production of particles in a $W$ target of $28.9 \mathrm{mg} / \mathrm{cm}^{2}$ and a $\mathrm{C}$ target of $50 \mathrm{mg} / \mathrm{cm}^{2}$ at $5^{\circ}$ angle with respect to the beam direction. A $2.2 \mathrm{GeV}$ electron beam was used with both targets. . . . . . . . . 148

5.1 Neutron spectrometer characteristics. Table was taken from Ref. [74].

5.2 Best fit parameters of second order polynomial fits for BSS count rates as a function of source-to-detector distance. . . . . . . . . . . . . . . 172

5.3 Best fit parameters of linear fits for BSS count rates as a function of source-to-detector distance. . . . . . . . . . . . . . . . . . 174 
5.4 A comparison of scatter-corrected count rates and absolute count rates is given. The scatter corrected count rates were calculated based on Bonner sphere measurments. The absolute count rates were calculated based on simulated Bonner sphere response and the known properties of the calibrated Am/Be source. . . . . . . . . . . . . . . . 175 


\section{List of Figures}

1.1 Parameter space for dark photons $\left(A^{\prime}\right)$ with mass $m_{A^{\prime}}>1 \mathrm{MeV}$. Here $\mathrm{y}$-axis is $\epsilon^{2}=\alpha^{\prime} / \alpha$, where $\alpha=e^{2} / 4 \pi$, fine structure constant for ordinary electromagnetic interactions. $\alpha^{\prime}$ is the fine structure constant for dark matter. Cross-sections of $A^{\prime}$ production scale as $\epsilon^{2}$. This parameter space can be probed by different proposed experiments and hown are existing $90 \%$ confidence level limits. The shaded areas indicate the results of already completed experiments and the lines indicate the area that will be covered by future experiments $[6][16]$. . . . . . .

1.2 $A^{\prime}$ production by bremstrahlung off an incoming electron scattering off protons in a target with atomic number Z. The $A^{\prime}$ particle could quickly decay into a $e^{+} e^{-}$pair. Figure taken from Ref. [31]. . . . . .

1.3 QED trident backgrounds. (a) radiative (b) Bethe-Heitler trident reactions that comprise the primary QED background to $A^{\prime} \rightarrow l^{+} l^{-}$. . 
1.4 The layout of the APEX test run [31]. An electron beam was incident on a thin tantalum foil target. Two septum magnets of opposite polarity deflected charged particles towards HRS spectrometers. The HRSs contain detectors to accurately measure the momentum, direction, and identity of the particles. Sieve slit plates located in front of the septum magnets were used for calibration of the spectrometer magnetic optics.

2.1 The layout of the experimental setup. . . . . . . . . . . . . .

2.2 Overall layout of the APEX target system. The target ladder consists of six sections from top to bottom; an optics calibration target, an alignment target, 3 production targets and empty section for beam studies. Note that the target system is elongated along the beam line. The beam passes through all the production ribbons, while outgoing electron/positron pairs in the HRS acceptance only pass through one

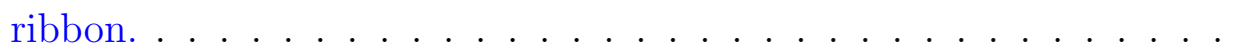

2.3 Left: Schematic illustration of the production ribbon holder. On one side of each holder is a tensioning bar that is spring loaded. This keeps the ribbons or wires straight when the material is hot. Right: A side view of production ribbon, beam-alignment wire and carbon optics holder. . . . . . . . . . . . . . . . . . .

2.4 The sketch illustrates the top view of the APEX setup. An electron leaving the target at an angle of $5^{\circ}$ with the positive beam direction, is deflected by the septum magnet towards the left HRS positioned at $12.5^{\circ} \ldots \ldots \ldots \ldots \ldots$

2.5 A side view (left) and a back view (right) of the fabricated septum magnet ready to be tested at JLab. . . . . . . . . . . . . . . 
2.6 A horizontal cut of the beam line components around the septum magnet as viewed from the top. . . . . . . . . . . . . .

2.7 A schematic cross section of Hall A with one of the HRS spectrometers in the (fictitious) $0^{\circ}$ position. Figure from Ref. [22]. . . . . . . .

2.8 Side view of detector stack for the left and right HRS. Individual elements of the detector system are indicated in the confguration used for APEX. The position of the data-acquisition(DAQ) electronics and of the VDC support frame are not shown. . . . . . . . . . . .

2.9 The particle identification with gas Cherenkov counter in the positron arm at the track rate of $765 \mathrm{kHz}$. The off-line pion rejection factor is 30.30

2.10 The total energy deposited in both layers pre-shower $\left(\mathrm{E}_{\mathrm{ps}}\right)$ and shower $\left(\mathrm{E}_{\mathrm{sh}}\right)$ of the calorimeter over the initial momentum of the incoming particles are shown. The first peak contains $\pi^{+}$events, the second contains $\mu^{+}$ events, and the third contains $e^{+}$events. GC has been used to calculate the efficiency of the calorimeter. . . . . . . . . . . . . 31

2.11 A schematic layout of Vertical Drift Chambers (VDC) for one HRS [22]. 32

2.12 Timing diagram of the coincidence trigger. The overlap of both $20 \mathrm{~ns}$ $\mathrm{S} 2 \mathrm{~m}$ pulses and the $10 \mathrm{~ns}$ right-HRS gas Cherenkov pulse creates a "golden" coincidence trigger. . . . . . . . . . . . . . . .

2.13 An event distribution of APEX test run vs. time between the electronarm S2m signal and the DAQ trigger signal. The total width of the accidental coincidence is $40 \mathrm{~ns}$. Even $20 \mathrm{~ns}$ trigger is possible. The real coincidence has a width of $10 \mathrm{~ns} \ldots \ldots \ldots \ldots \ldots \ldots$

2.14 The invariant mass spectrum of $e^{+} e^{-}$pair events in the APEX test run. APEX test run searched for an $A^{\prime}$ particle in the mass range of $175-250 \mathrm{MeV}$. No hints for an $A^{\prime}$ was found in this mass window. . 
3.1 Plots illustrate photon, electron and neutron production from $11 \mathrm{GeV}$ electron beam incident on a $1 \%$ r.l. Carbon target. Plots a(1), b(1) and $c(1)$ were generated using a GEANT3 simulation and a(2), b(2) and c(2) were generated using GEANT4 simulation developed for this study. In all plots, y-axis indicates the number of events per $\mathrm{MeV}$ and per unit sold angle normalized by number of incident electrons. X-axis is the kinetic energy of particles leaving the target. Plots with different colors separate particles by its opening angle $\theta$ with respect to beam

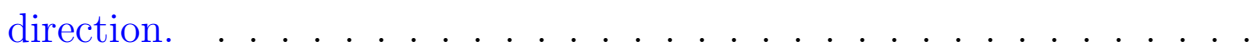

3.2 Geant4 model of hall A used by PREX collaboration for their simulations $[89] \ldots \ldots \ldots \ldots \ldots \ldots \ldots$

3.3 This figure illustrates the Geant4 model of JLab hall A. Top and Bottom images show the same geometry with different features labeled. .

3.4 Geant4 model of the downstream beamline in JLab hall A. Different colors are used to indicate different sections of the beamline. The numbered labels correspond to the raws in the Table 3.2 . . . . . . . 46

3.5 A side view of a high resolution spectrometer in JLab hall A. The components that were included in the Geant4 model are labeled. . . . 48

3.6 A front view of the Geant4 model of Hall A as seen from the target. . 49

3.7 A side view of the Geant4 model of a Hall A HRS spectrometer. . . . 49

3.8 A top view of the Geant4 model of APEX setup. Electrons and positrons leaving the target at small angles are bent by the septum magnet towards the High Resolution Spectrometers placed at $12.5^{\circ}$ with respect to the beamline. . . . . . . . . . . . . 51

3.9 Geant4 model of APEX extension box. . . . . . . . . . . . . . 51 
3.10 The septum magnet which will be used in the APEX experiment is shown here. Currently it is at JLab undergoing testing. . . . . . . . . 52

3.11 This illustrates the PREX-I septum magnet. . . . . . . . . . . . . . . 52

3.12 The Geant4 model of the APEX septum magnet that was constructed for the APEX experiment. Most of the parts in the magnet is iron and the coils are made of copper. TOSCA field maps were used to apply magnetic field in to the septum magnet. . . . . . . . . . . . .

3.13 This illustrates TOSCA field map for APEX $4.4 \mathrm{GeV}$ setting. The y component of the magnetic field along the $\mathrm{x}$-axis is shown in the plot. The image of the septum magnet in the background and the coordinate system used in the simulation are provided as a guide. . . . . . . . . 54

3.14 This illustrates TOSCA field map used for the PREX-I experiment. The y component of the magnetic field along the $\mathrm{x}$-axis is shown in the plot. The image of the septum magnet in the background and the coordinate system used in the simulation are provided as a guide. . .

3.15 The Geant4 model of the PREX extension box. The Tungsten collimator was placed inside this extension box. . . . . . . . . . . 56

3.16 The Geant4 model of the PREX collimator. . . . . . . . . . . . 56

3.17 This illustrates the PREX-I experiment setup. The tungsten collimator sits inside the PREX-I extension box. Some details of the HRS spectrometer are removed for clarity of the image. . . . . . . . . .

3.18 Geant4 model of the RCS experiment. The model was simplified by not including the deflection magnet and the calorimeter. Neither an extension box nor a septum magnet was used in the experiment. . . . 
3.19 This figure shows the scoring-volumes used in the Geant4 model. A semi-circular shaped scoring-volume was used in the simulation to detect particles in the upstream region. We used a large volume to increase statistics. A $2 \mathrm{~m} \times 2 \mathrm{~m} \times 2 \mathrm{~m}$ box was used as the scoring-volume in downstream region. . . . . . . . . . . . . . . . .

3.20 Silicon displacement damage functions for neutrons, protons, electrons and pions. Values are normalized to the displacement damage by 1 $\mathrm{MeV}$ neutrons $(95 \mathrm{MeVmb}) . \ldots \ldots \ldots \ldots$

3.21 A rough estimate for $1 \mathrm{MeV}$ neutron fluence thresholds for different material/electronics. . . . . . . . . . . . . . . .

3.22 Drawing illustrates the top view of the Jefferson Lab experimental hall A equipment. Most of the radiation sensitive semiconductor electronics are located at the position labeled as "HRS control and power electronics". This is the main point where we evaluated radiation damage. . .

3.23 Figure shows a photograph of the HRS with a view of the HRS control electronics. Each HRS has its own control electronics at the back of the HRS just under the shield hut. This area also moves with the HRS when it rotates around the hall A pivot. . . . . . . . . . . . 
3.24 The dashed (black) line shows the simulated neutron fluence-rate spectrum at the location of the HRS control electronics for the APEX experiment with $3.3 \mathrm{GeV}$ setting. Beam current was assumed to be $120 \mu \mathrm{A}$. The histogram has equal-width bins in the log scale and the fluence is per energy bin which is indicated by $\Phi(\mathrm{E}) \mathrm{dE}$. The solid (red) line represents the original (black) neutron spectrum weighted by the Silicon damage curve. The dotted (blue) line shows the cumulative silicon damage as a percentage and it is indicated by the secondary axis on the right side of the plot. . . . . . . . . . . . .

3.25 The dashed (black) line shows the simulated electron fluence-rate spectrum at the location of the HRS control electronics for the APEX experiment with the $3.3 \mathrm{GeV}$ setting. Beam current was assumed to be $120 \mu \mathrm{A}$. The histogram has equal-width bins in the log scale and the fluence is per energy bin which is indicated by $\Phi(\mathrm{E}) \mathrm{dE}$. The solid (red) line represents the original (black) electron spectrum weighted by the Silicon damage curve. The dotted (blue) line shows the cumulative silicon damage as a percentage and it is indicated by the secondary axis on the right side of the plot. . . . . . . . . . . . .

3.26 Shown is the simulated photon fluence-rate spectrum at the HRS control electronics for the APEX experiment with the $3.3 \mathrm{GeV}$ setting. Beam current was assumed to be $120 \mu \mathrm{A}$. The histogram has equalwidth bins in the log scale and the fluence is per energy bin which is

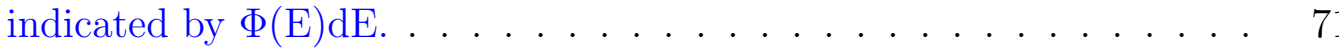


3.27 A side view (top) and a top view (bottom) of the APEX simulation setup are shown. Four Geant4 scoring volumes were placed in the hall A at four different locations (labeled in the figure) to evaluate radiation levels in terms of $1 \mathrm{MeV}$ equivalent neutron fluence. The circle with a dashed line indicates an arbitrary distance of $2 \mathrm{~m}$. For the APEX experiment, the radiation levels are already below the ESAD threshold at this distance. . . . . . . . . . . . . . . . . .

3.28 The simulated neutron spectrum in upstream region of Hall A for the APEX experiment. Events were binned by neutron energy. The histogram has equal-width bins in the log scale and the fluence is per energy bin which is indicated by $\Phi(\mathrm{E}) \mathrm{dE}$. We considered a beam current of $50 \mu \mathrm{A}$ for the calculation. . . . . . . . . . . . . . .

3.29 This plot shows the conversion coefficients for neutron fluence to neutron dose as a function of neutron energy. . . . . . . . . . . .

3.30 This diagram shows the positioning of NP100B (SNOOPY) in Hall A during the PREX-I and RCS experiments. In both cases SNOOPY's were placed in upstream region close to the entrance of Hall A. . . . .

3.31 Measured neutron dose rates in the upstream of the JLab Hall A using an NP100B neutron probe during the PREX-I experiment. Dose rates in the plot were measured at different beam currents and were normalized to a $100 \mu \mathrm{A}$ beam current. . . . . . . . . . . .

3.32 Measured neutron dose rates in the upstream of the JLab Hall A using an NP100B neutron probe during the RCS experiment. Dose rates in the plot were measured at different beam currents and were normalized

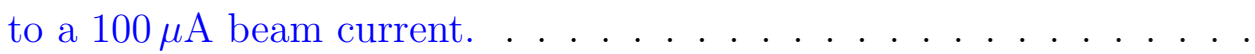


3.33 Simulated neutron fluence rates in the upstream of the JLab Hall A during the RCS experiment. We assumed a beam current of $40 \mu \mathrm{A}$. Events are binned by neutron energy. The histogram has equal-width bins in the log scale and the fluence is per energy bin which is indicated by $\Phi(\mathrm{E}) \mathrm{dE}$. . . . . . . . . . . . . . . . . . . . .

3.34 An exploded view of the SNOOPY. The outer polyethylene layer, Attenuators made of boron doped rubber and the proportional counter filled with $\mathrm{BF}_{3}$ gas are shown here. . . . . . . . . . . .

3.35 The original figure of this is from [81]. It compares the FLUKA calculation of the SNOOPY response to neutrons with measurements. The SNOOPY response calculated using Geant4 in this study is plotted in the same figure for easy comparison. . . . . . . . . . . . . .

3.36 Identification of the primary vertices in the APEX experiment. When a particle track enters the HRS-L volume, it is tracked back to its primary vertex using an algorithm. The identified vertices of track A, $\mathrm{B}$ and $\mathrm{C}$ are $\mathrm{V} 1, \mathrm{~V} 2$ and $\mathrm{V} 3$ respectively. . . . . . . . . . . .

3.37 Simulated power deposition in the HRS-L volume due to $\gamma$ radiation for the APEX 3.3 GeV experiment. Each pixel in the 2D plot indicates a group of primary vertices ( $\mathrm{x}$ and $\mathrm{z}$ coordinates) and the color of a pixel represents how much energy was deposited in the HRS-L volume from the $\gamma$ rays originated from those vertices. . . . . . . . . .

3.38 Simulated power deposition in the HRS-L volume due to neutrons for the APEX 3.3 GeV experiment. Each pixel in the 2D plot indicates a group of primary vertices ( $\mathrm{x}$ and $\mathrm{z}$ coordinates) and the color of a pixel represents how much energy was deposited in the HRS-L volume from the neutrons originated from those vertices. . . . . . . . . . . 
3.39 Simulated power deposition in the HRS-L volume due to neutrons for the APEX 3.3 GeV experiment. Y-axis shows the deposited power per each bin in the histogram and the $\mathrm{x}$-axis shows the $\mathrm{z}$ coordinate of a primary vertex. Events in the histogram were limited to the vertices with an $\mathrm{x}$ coordinate in the range $-100 \mathrm{~cm}<\mathrm{x}<100 \mathrm{~cm}$. . . . . . . 97

3.40 Simulated power deposition in the HRS-L volume due to $\gamma$ for the APEX $3.3 \mathrm{GeV}$ experiment. Each pixel in the 2D plot indicates a group of primary vertices ( $\mathrm{x}$ and $\mathrm{z}$ coordinates) and the color of the pixel represents how much energy was deposited in the HRS-L volume due to the $\gamma$ rays originated from those vertices. This is a blown-up

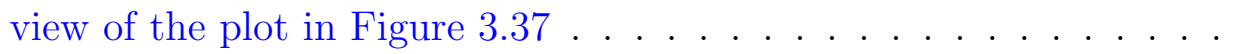

3.41 Simulated power deposition in the HRS-L volume due to neutrons for the APEX 3.3 GeV experiment. Y-axis indicates the deposited power normalized by the bin size of the histogram and the $\mathrm{x}$-axis indicates the $\mathrm{z}$ coordinate of the primary vertices. Events in the histogram were limited to the vertices with an $\mathrm{x}$ coordinate in the range $-100 \mathrm{~cm}<$ $\mathrm{x}<100 \mathrm{~cm}$. This is a blown-up view of the plot in Figure 3.39. . . . . 100

3.42 The photograph shows a top view of the setup around the telescoping beam pipe. Simulations identified that the most of the radiation reached the HRS-L scoring volume was originated in this section of the beam line. . . . . . . . . . . . . . . . . .

3.43 A top view of the left HRS. The beam line, components of the HRS and the HRS-L scoring volume can be seen in the image. The HRS-L scoring volume consists of 40 layers in the $z^{\prime}$ direction and 10 layers in the $\mathrm{x}^{\prime}$ direction. . . . . . . . . . . . . . . . . . . . 102

3.44 A 3D view of the HRS-L scoring volume. . . . . . . . . . . . . 103 
3.45 Electron dose distribution within the HRS-L scoring volume. Dose in each $z^{\prime}$-layer is plotted as a function of $x^{\prime}$-layer number. Only $z^{\prime}$-layers with significant dose difference are shown here. . . . . . . . . . . . 105

3.46 Photon dose distribution within the HRS-L scoring volume. Dose in each $z^{\prime}$-layer is plotted as a function of $x^{\prime}$-layer number. Only z'-layers with significant dose difference are shown here. . . . . . . . . . . . . 106

3.47 Neutron dose distribution within the HRS-L scoring volume. Dose in each $z^{\prime}$-layer is plotted as a function of $x^{\prime}$-layer number. Only $z^{\prime}$-layers with significant dose difference are shown here. . . . . . . . . . . . . . 107

3.48 Tungsten collimator that is used to focus the beam towards the narrow section of the beam line (left). This collimator will be placed inside the magnetic beam pipe (right) . . . . . . . . . . . . . . . 108

3.49 A horizontal cross-section of the septum magnet as seen from the top. The tungsten collimator is visible and it is located about $20 \mathrm{~cm}$ from the upper edge of the septum magnet. . . . . . . . . . . . .

3.50 APEX 3.3 GeV at $120 \mu \mathrm{A}$ beam current was simulated with and without the Tungsten collimator. Energy spectra of neutrons at HRS-L electronics area were compared. There is a reduction of neutrons above $0.1 \mathrm{MeV}$ when the collimator is being used. . . . . . . . . . . . . . . 110

3.51 APEX $3.3 \mathrm{GeV}$ at $120 \mu \mathrm{A}$ beam current was simulated with and without the Tungsten collimator. Energy spectra of neutrons in upstream were compared. There is a increase of neutrons when the collimator is being used. . . . . . . . . . . . . . . . . . . . . . 111

4.1 A model of a Tungsten sieve plate. . . . . . . . . . . . . . . 118 
4.2 During a calibration run, the sieve plate is positioned between the target and the entrance to spectrometer. Analyzing the reconstructed and surveyed hole locations in the sieve plane, the reconstruction matrix can be optimized. . . . . . . . . . . . . . . . . . .

4.3 The geometric (left) and reconstructed (right) configurations of the sive slit. The large holes helps to identify the orientation of the image at the focal plane. . . . . . . . . . . . . . . . . .

4.4 The $\mathrm{x}$ and $\mathrm{y}$ distributions for a single sieve hole. The distributions were fit with a guassian (black) on top of a linear background (red). The blue line indicates the surveyed location of the hole. The mean and the sigma of the fit correspond to the reconstructed position and width of the hole $[71][31] . \ldots \ldots \ldots$. . . . . . . . . . . . . . . . . 121

4.5 The active area of the Scintillating Fiber (SciFi) detector. . . . . . . 123

4.6 All 64 fiber ends are threaded through two guide brackets (A and B) and connected to a separator $(\mathrm{C}) \ldots \ldots \ldots \ldots$

4.7 Two fiber separators. Scintillating fiber separator is on the left and optical fiber separator on the right. . . . . . . . . . . . . . 125

4.8 Complete setup of the SciFi detector. SciFi detector is connected to an mechanical arm driven by a stepper motor. Scinitllating fibers are coupled to clear optical fibers through two separators. The long 64 clear optical fibers guides the scintillation light to the readout electronics and maPMT. . . . . . . . . . . . . . . . . . 125

4.9 A 3D drawing of the hall A layout. Two SciFi detectors will be used, one for each HRS which are connected to the extension box through a vacuum feed-through. SciFi actuators are stepper motors which will be used to move the SciFi in and out of the beam. . . . . . . . . . . . 126 
4.10 Custom light tight box constructed for the experiment. SciFi detector, Trigger counter and the radiation source were housed inside the dark box. A PMT was connected from outside of the box. . . . . . . . . . 128

4.11 Inside of the dark box used for the experiment. Active area of the SciFi and the scintillating block of the trigger counter are placed parallel to each other. PMT of the trigger counter is inside the box. Radiation source was placed very close to the SciFi detector surface. . . . . . . . 129

4.12 Outside of the dark box. An aluminum cylindrical structure was connected to the box from outside to hold the 2-inch PMT. The PMT and the scintillating fiber ends are touching inside the aluminum structure. 130

4.13 Block diagram of the experiment setup. . . . . . . . . . . . . . . . 131

4.14 Example of a SciFi pulse height spectrum. The Pedestal peak is due to DAQ system getting triggered even when no signal is present. Rest of the spectrum is due to single or multiple photo-electron events. . . 132

4.15 Upper half of the scintillating fiber with a circular cross section. A particle track passes the fiber vertically at distance $x$ from the center. The path length of the particle through the fiber is $\sqrt{r^{2}-x^{2}}$. . . . 134

4.16 The distribution of photoelectrons in the PMT (used with the SciFi) is given by the probability function $B_{n}\left(\mu_{0}\right)$. Calculations of $B_{n}\left(\mu_{0}\right)$ for only $n=3$ and $n=10$ as a function of $\mu_{0}$ are shown in the figure. . . 137

4.17 Pulse height spectrum of SciFi detector when 2-inch PMT was connected directly to the dark box. The equation 4.25 was used to fit the distribution. This has a poor fit to the single photo-electron peak. . . 138 
4.18 Pulse height spectrum of SciFi detector when 2-inch PMT was connected directly to the dark box. The equation 4.26 was used to fit the distribution. Fit to the single photo-electron peak is improved by introducing 2 more parameters acc1 and acc2 to represent accidental events. . . . . . . . . . . . . . . . .

4.19 The energy deposition in scintillating fibers of SciFi detector was simulated using Geant4 package. The left image shows the 2-layer arrangement of scintillating fibers. The image on the right shows a snapshot taken during the simulation. . . . . . . . . . . . . . . .

4.20 In the histogram, events are binned by the total energy deposited in all 64 fibers. The peak around $160 \mathrm{keV}$ of deposited energy (single fiber pass) resulted from electrons passing through a single fiber. The other peak around $300 \mathrm{keV}$ (double fiber pass) is due to electrons passing through a crossing of two fibers. . . . . . . . . . . . . . . . . . 141

4.21 The distribution of photoelectrons in the PMT (used with the SciFi) is given by the probability function $B_{n}\left(A_{0}\right)$. Calculations of $B_{n}\left(A_{0}\right)$ for only $n=0$ to $n=5$ as a function of $A_{0}$ are shown in the figure. $\quad 143$

4.22 Pulse height spectrum of SciFi detector when 2-inch PMT was connected directly to the dark box. The equation 4.29 was used to fit the distribution. The probability function $B_{n}\left(\mu_{0}\right)$ was calculated based on the true response of the SciFi detector. . . . . . . . . . . . . . . . 144

4.23 A repeating element of the active area of the SciFi detector. This was used to calculate the ratio of the area of fiber to the area of empty spaces. . . . . . . . . . . . . . . . . . 144 
4.24 Pulse height spectrum of SciFi detector when 2-inch PMT was connected directly to the dark box. We used a trigger counter with smaller area and thickness compared to the setup which produced the spectrum

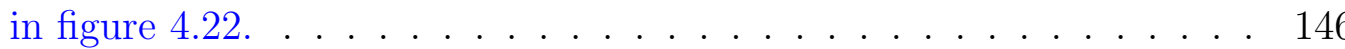

4.25 Pulse height spectrum of SciFi detector when 2-inch PMT was connected via the long optical cable. The source of extra events in the high energy tail is unknown. . . . . . . . . . . . . . . . . . . 147

4.26 Electron production in a Tungsten target of thickness $28.9 \mathrm{mg} / \mathrm{cm}^{2}$ with an electron beam of $2.2 \mathrm{GeV}$ energy. $\mathrm{Y}$ axis is the number of events per solid angle per each electron incident on the target. $\mathrm{X}$ axis is the angle of electrons exiting the target with respect to the beam direction. ...................... . . 149

4.27 Electron production in a Carbon target of thickness $50 \mathrm{mg} / \mathrm{cm}^{2}$ with an electron beam of $2.2 \mathrm{GeV}$ energy. $\mathrm{Y}$ axis is the number of events per solid angle per each electron incident on the target. $\mathrm{X}$ axis is the angle of electrons exiting the target with respect to the beam direction. 150

5.1 Response functions of original Bonner sphere set. These are counts per $10^{6}$ neutrons emitted from an isotropic point source $40 \mathrm{~cm}$ away. This figure was taken from the Ref. [75] . . . . . . . . . . . . . 157

$5.2{ }^{3} \mathrm{He}(\mathrm{n}, \mathrm{p}),{ }^{10} \mathrm{~B}(\mathrm{n}, \alpha)$ and ${ }^{6} \mathrm{Li}(\mathrm{n}, \alpha)$ cross sections as a function of incident

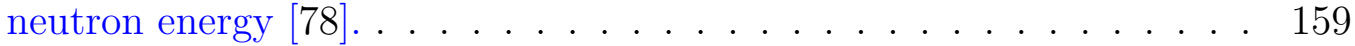

5.3 A typical pulse-height spectrum for a thermal neutrons detected by a ${ }^{3}$ He filled counter [78]. . . . . . . . . . . . . . . . . . . . . . . . 160 
5.4 Response functions of Bonner sphere spectrometer set calculated with GEANT4. Gold foil is used inside of spheres as the thermal neutron detector. Sphere size in inches is indicated on each line [83]. . . . . . 161

5.5 A cross-sectional side view of one of the Bonner spheres. The diameter of the sphere is 10 inches and there is a 0.7 -inch thick lead layer inside of the sphere. The spherical proportional counter is filled with $10 \mathrm{~atm}$ ${ }^{3}$ He gas. . . . . . . . . . . . . . . . . . . . .

5.6 The Bonner sphere spectrometer at Jefferson Lab contains 5 separate spheres. The diameters of the spheres are 5-inch, 8-inch, 10-inch and 12-inch. There is an extended Bonner sphere of 10-inch diameter with a $\mathrm{Pb}$ layer. . . . . . . . . . . . . . . . . . . . 163

5.7 Block diagram of the experimental setup. . . . . . . . . . . . . . . . 164

5.8 Dimensions of the detector calibration room at Jefferson Lab. The drawing on the left shows the top view of the room and the drawing on the right shows a side view of the back wall constructed of concrete masonry unit (CMU). The height of the room is $12 \mathrm{ft}$ and the height

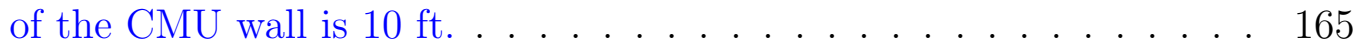

5.9 A photograph of the calibration room at JLab. The wall constructed of concrete masonry units (CMU) is visible and the controls of the steel conveyor and the neutron source is located behind the CMU wall. Neutron source is located below the ground and moves within the PVC pipe shown in the photograph. . . . . . . . . . . . . 166

5.10 A photograph of the calibration room at JLab. The detector platform can move along the steel conveyor, up/down or left/right. The maximum source-to-detector distance is $\sim 5 \mathrm{~m}$. . . . . . . . . . 
5.11 Experimental setup for BSS calibration. 12-inch sphere is mounted on the platform. . . . . . . . . . . . . . . . . . 169

5.12 (left) Pulse height spectra from all the Bonner spheres located $1 \mathrm{~m}$ from the Am-Be neutron source. (right) Neutron rate for the corresponding sphere in Hz. . . . . . . . . . . . . . . . . . . . . . . . . . . 170

5.13 Measured count rates using Bonner spheres are multiplied by $d^{2}$ and plotted as a function of source-to-detector distance $d$. The data were fitted using a second order polynomial. Best fit parameters are indicated in Table $5.2 . \ldots \ldots \ldots \ldots \ldots$

5.14 The Geant4 model of the JLab detector calibration facility. Steel walls of the room, A concrete wall, concrete floor, A PVC pipe, steel conveyor and a Bonner sphere is visible in the illustration. The roof and the $6 \mathrm{~m}$ thick soil beneath the floor of the facility is removed for illustration

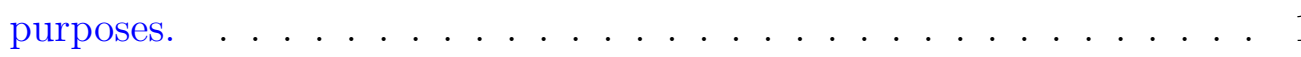

5.15 IAEA Am-Be neutron spectrum. The spectrum is constructed using 60 energy points and it is normalized. . . . . . . . . . . . . . . . 178

5.16 Measured and the simulated count rates in the $12^{\prime \prime}$ diameter Bonner sphere. The y-axis is the count rate multiplied by the square of the distance. The x-axis is the source-to-detector distance. The errors on the blue points are statistical errors from the simulation. The data were fit to a linear equation. . . . . . . . . . . . . . . . . . . 180

5.17 Measured and the simulated count rates in the $5^{\prime \prime}$ diameter Bonner sphere. The y-axis is the count rate multiplied by the square of the distance. The $\mathrm{x}$-axis is the source-to-detector distance. The errors on the blue points are statistical errors from the simulation. The data were fit to a linear equation. . . . . . . . . . . . . . . . . 181 
5.18 Calculation of count rates in the $12^{\prime \prime}$ diameter sphere in different environments. The y-axis of the plot is count rate multiplied by the square of distance and the $\mathrm{x}$-axis is the source-to-detector distance. The black triangles represent calculated values when the Bonner sphere is in low density gas (Vacuum). The red circles represent values when the calibration room was included and the blue squares indicate values when dry air was added. . . . . . . . . . . . . . . . . 182

5.19 Calculation of count rates in the $5^{\prime \prime}$ diameter sphere in different environments. The y-axis of the plot is count rate multiplied by the square of distance and the $\mathrm{x}$-axis is the source-to-detector distance. The black triangles represent calculated values when the Bonner sphere is in low density gas (Vacuum). The red circles represent values when the calibration room was included and the blue squares indicate values when dry air was added.

5.20 Pulse height spectra generated from the simulation of 12-inch Bonner sphere with $1 \mathrm{keV}$ neutrons (left) and $63 \mathrm{MeV}$ neutrons (right) from a point source located $1 \mathrm{~m}$ away from the detector. . . . . . . . . . . 185

5.21 Calculated response functions using Geant4 simulation package. Response of the bare detector was scaled by 0.7 . . . . . . . . . . 186

A.1 The dimensions and the components of the NP100B (SNOOPY) neutron detector. . . . . . . . . . . . . . . . . . 205

A.2 A cross section view of the SNOOPY detector. The image is taken from the Geant4 model we developed. . . . . . . . . . . . . . . . . . 206

A.3 An exploded view of the SNOOPY. The front attenuator is not shown in the picture. . . . . . . . . . . . . . . 206 
A.4 A detailed view of the rear attenuator of the SNOOPY detector. The thickness of the cicular shaped attenuator is $0.635 \mathrm{~cm}$. The holes with diameter of $0.95 \mathrm{~cm}$ are through holes. . . . . . . . . . . . . . 207

A.5 A detailed view of the attenuator sleeve. Wall thickness of the sleeve is $0.635 \mathrm{~cm}$. The holes with diameter of $0.95 \mathrm{~cm}$ are through holes. . . 207

A.6 A detailed view of the attenuator at the front of the detector. The thickness of the cicular shaped attenuator is $0.635 \mathrm{~cm}$. The holes with diameter of $0.95 \mathrm{~cm}$ and the large center hole with a diameter of $2.85 \mathrm{~cm}$ are through holes. . . . . . . . . . . . . . . . . . . . 208

A.7 The response function of the SNOOPY calculated using Geant4 simulations is compared with the similar calculations by Tessler et. al. The $\mathrm{y}$-xis of the plot is the counts per unit fluence and the $\mathrm{x}$-axis is the

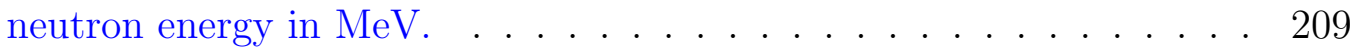

B.1 Reconstructed Am-Be spectrum using MLEM algorithm. A constant value was chosen for all the energy bins as the initial guess spectrum. 640097 iterations were taken to reconstruct this spectrum. Reference Am-Be spectrum is also shown here. . . . . . . . . . . . . . . . . . . 212

B.2 Reconstructed Am-Be spectrum using MLEM algorithm. Guess spectrum bin values were set to zero except in the bins between $0.1 \mathrm{MeV}$ and $10 \mathrm{MeV}$. 687973 iterations were taken to reconstruct this spectrum. Reference Am-Be spectrum is also shown here. . . . . . . . . . 213 


\section{Chapter 1}

\section{Introduction}

The $A^{\prime}$ (A-prime) experiment (APEX) which is approved to run at Jefferson Laboratory (JLab) will search for a hypothetical particle known as an $A^{\prime}$. The existence of so-called dark matter is widely accepted, and the possible existance of an $A^{\prime}$ has been postulated as the carrier of a new force between dark-matter particles. In this experiment, an electron beam of $1-4 \mathrm{GeV}$ with an beam current of $\sim 100 \mu \mathrm{A}$ will be incident on a $5.3 \%$ radiation length Tungsten target. The $e^{+} e^{-}$pairs leaving at a nominal angle of $5^{\circ}$ will be detected in High Resolution Spectrometers (HRS) in Hall A. The signal due to an $A^{\prime}$ will appear on top of a background of $e^{+} e^{-}$events and a "bump-hunt" needs to be carried out to identify the $A^{\prime}$ particle.

The "Standard Model" of elementary particles and forces provides a theoretical framework that is consistent with all known experimental data. In the Standard Model, subatomic particles interact via electromagnetic, strong and weak forces that are mediated by force carriers called "gauge bosons". New forces could exist beyond our reach either because the force-carrying particles are heavier than $\mathcal{O}(\mathrm{TeV})$ or because they are very weakly coupled to ordinary matter. Since no evidence for new physics has been seen at high energies, physicists have been designing high-statistics 
and high-precision measurements that are sensitive to very weak effects. APEX is such an experiment.

Experiments that are high luminosity and high sensitivity present many technical challenges. This thesis focuses on several technical challenges that needed to be resolved in order to progress towards the APEX experiment. One such challenge is to evaluate and perhaps lower the radiation risk to semiconductor electronics in the experimental hall.

\subsection{The challenge of high radiation experiments}

Electron scattering experiments generally produce high radiation environments near and around targets. The ${ }^{208} \mathrm{~Pb}$ radius experiment (PREX-I) which ran in JLab's Hall A in 2010 and the APEX experiment which was approved to run in JLab's Hall A in 2018 are examples of such high radiation experiments. Modern semiconductor electronics used in experimental halls are mostly Silicon based, which makes them vulnerable to radiation damage. For example, radiation during the PREX-I experiment did significant damage to spectrometer electronics and provided us with a valuable measure of radiation levels that are threatening to the safety of equipment. In fact, as will be discussed later the radiation levels we expect during APEX will be about a factor of 9 smaller than the radiation levels that were present during PREX-I.

Monte Carlo simulation packages, which includes Geant3 [1], Geant4 [2] and FLUKA [3], provide a way of understanding what radiation levels to expect during high-radiation experiments. These modern simulation packages have the capability to accurately model a wide variety of experimental setups. The simulations include so-called "Physics lists" which describe most of the important physics processes, thus allowing us to simulate particle interactions accurately. The simulations make it 
possible to establish relative comparisons of radiation levels between different experiments. The simulations also allow the computation of radiation levels that can be compared with accumulated knowledge of the effects of radiation.

Calculating radiation is not trivial, so an important question is how to go about believing your results. One can start by calculating radiation using a simple geometry and comparing the results with other accepted simulations. We performed such checks, comparing our results with simulations of simple geometries that were also simulated by the Radiation Control Department (RadCon) at JLab. We also simulated PREX-I, and compared our results with existing simulations performed by the PREX collaboration. Comparing simulations with empirical measurements can also provide increased confidence, and we present such absolute comparisons. All of the checks put us in a position to calculate what to expect for future experiments, such as APEX.

Another challenge is, if the radiation levels are expected to be too high, determining how to properly shield areas where sensitive equipment is located. We also examine such possibilities for APEX, although it is not clear that additional shielding is needed.

\subsection{The physics of APEX}

The existence of dark matter provides powerful evidence for the incompleteness of the Standard Model. According to the standard model of cosmology, known as the $\Lambda \mathrm{CDM}$ model, dark matter dominates the matter density in the universe, but very little is known about it [5]. The $\Lambda \mathrm{CDM}$ model indicates that the total mass-energy of the universe contains $4.9 \%$ of ordinary matter, $26.8 \%$ of dark matter and $68.3 \%$ of dark energy. Dark matter provides a strong hint that there could be a dark sector, 
consisting of particles that do not interact with the strong, weak or electromagnetic forces [6]. These dark sectors may contain light and very weakly-coupled particles that mediate "dark forces" that also interact very feebly with ordinary matter. New and powerful experiments are underway to probe these new hidden dark sectors.

\subsubsection{Motivation for the JLab APEX experiment}

Recent cosmological observations hints that dark matter may couple to Standard Model matter through exchange of an $A^{\prime}$.

The satellites PAMELA [23] and Fermi [24], the balloon-bourne detector ATIC [25], the terrestrial-based telescope HESS [26] have observed a large excess in the cosmic-ray flux of high-energy electrons and positrons relative to what is expected from normal astrophysical processes. If dark matter is composed of weakly-interacting massive particles (WIMP's) with an energy of $10 \mathrm{GeV}$ to $10 \mathrm{TeV}$ that interact via the electro-weak force, this could explain the relic abundance of dark matter observed in our universe today. This is referred to as the "WIMP miracle". But, the annihilation cross-section of WIMP's that is required to explain the WIMP miracle is 50-1000 times smaller than what is required to explain the $e^{+} e^{-}$excess [28]. If the dark matter interacts with an $\mathcal{O}(\mathrm{GeV})$-mass $A^{\prime}$, the dark matter annihilation rate would be enhanced and would explain the excess of positrons and electrons.

In addition to dark matter anomalies, the existence of an $A^{\prime}$ might also help to explain other physics anomalies. The magnetic moment of the muon can be expressed using the equation

$$
\vec{M}=g_{\mu} \frac{e}{2 m_{\mu}} \vec{S}
$$

where $g_{\mu}$ is the g-factor, $e$ is the electron charge, $m_{\mu}$ is the muon mass and $\vec{S}$ is the 
spin angular momentum. The Dirac equation predicts $g_{\mu}=2$. Radiative corrections introduce an anomalous magnetic moment, $a_{\mu}$, defined by,

$$
a_{\mu}=\frac{g_{\mu}-2}{2}
$$

The experimental measurements of $a_{\mu}$ disagree with Standard Model predictions by $2.2-2.7$ standard deviations [29]. The existence of a new force mediator that couples to muons, like the $A^{\prime}$, is one possible explanation for the $a_{\mu}$ discrepancy. In Figure 1.1 the $A^{\prime}$ s in the green band (see top left in the plot) can explain the observed discrepancy between the calculated and measured muon anomalous magnetic moment [30] at $90 \%$ confidence level. In general, there has never been a systematic search for the new GeV-scale force carriers that are weakly coupled to Standard Model particles. The discovery of such a particle would represent a breakthrough in our understanding the longstanding dark matter mystery and would have profound implications for our understanding of nature.

\subsubsection{The $A^{\prime}$ boson as a probe of dark matter}

The $A^{\prime}$ experiment (APEX), proposed to run at the Thomas Jefferson Accelerator Facility (JLab), will search for a new sub-GeV mass vector boson that couples very weakly to electrons through "kinetic-mixing" [7][8][10]. This new boson is usually referred to as the $A^{\prime}$ (A-prime), but it is also often called a "dark photon", "heavyphoton" or "U-boson". Kinetic mixing ${ }^{\dagger}$ between the $A^{\prime}$ and the photon produces an interaction of the form $\epsilon e A_{\mu}^{\prime} J_{E M}^{\mu}$. Here, $J_{E M}^{\mu}$ is the electromagnetic current and the parameter $\epsilon$ indicates the suppression of the interaction relative to the charge of the

\footnotetext{
${ }^{\dagger}$ Kinetic mixing is a phenomenon where the mass eigenstates and interactions of the vector bosons change.[9]. The kinetic mixing angle modifies the coupling of the corresponding gauge bosons and can therefore lead to observable effects [8]
} 
electron $e$. We often denote the coupling strength as $\alpha^{\prime} \equiv \epsilon^{2} \alpha$, where $\alpha=e^{2} / 4 \pi \simeq$ $1 / 137$, a dimensionless quantity. $\alpha^{\prime}$ is the effective fine structure constant for the dark photon. The values in the range $\sim 10^{-23}-10^{-2}$ have been predicted for $\epsilon$ in the literature [11]. The cross-sections for $A^{\prime}$ production scales as $\epsilon^{2}$. Depending on the mechanism from which the $A^{\prime}$ acquires mass, the $A^{\prime}$ can have a large range of values for its mass $\left(m_{A^{\prime}}\right)$. Figure 1.1 illustrates a parameter space for the $A^{\prime}$ particle spanned by different values for both $\epsilon^{2}$ and $m_{A^{\prime}}$. The wide range of possible values for both $\epsilon$ and the $m_{A^{\prime}}$ calls for multiple experimental approaches.

There are many experimental approaches to find the dark photon. Electron beamdump experiments [13], electron fixed-target experiments [7] and positron-electron collider experiments [20] are a few of them. In electron beam-dump experiments (quite different than the approach of APEX); a high-intensity electron beam dumps its energy into a fixed target, which provides the large luminosities that are needed to probe the weak coupling of the dark photon. Detectors are placed behind shielding that is sufficient to shield Standard Model backgrounds. The dark photons, with a decay length of $\mathcal{O}(\mathrm{cm}-\mathrm{m})$, produced at the target due to their very weak interaction with the Standard Model particles can penetrate the shielding and reach the detectors. This method can be used to detect dark photons with mass larger than $2 m_{e}$ up to $\mathcal{O}(100) \mathrm{MeV}$ and small values of $\epsilon$, roughly $10^{-7} \leq \epsilon \leq 10^{-3}$. Examples for these types of experiments are E141 [13] and E137 [12] at SLAC, E774 [14] at Fermilab, and an experiment in Orsay [15]. Electron beam dump experiments cover the lower left part of the parameter space shown in Figure 1.1. Fixed target experiments use high-current electron beams to search for an $A^{\prime}$ with masses $2 m_{e}<m_{A^{\prime}}<1 \mathrm{GeV}$ and couplings as small as $\epsilon^{2} \sim 10^{-10}[6]$. In most of these experiments the decay products of the $A^{\prime}, e^{+} e^{-}$pairs, will be detected in two separate spectrometers. APEX [7], HPS [17] and DarkLight [18] at JLab, and A1 [19] using the MAMI and MESA accelerators 


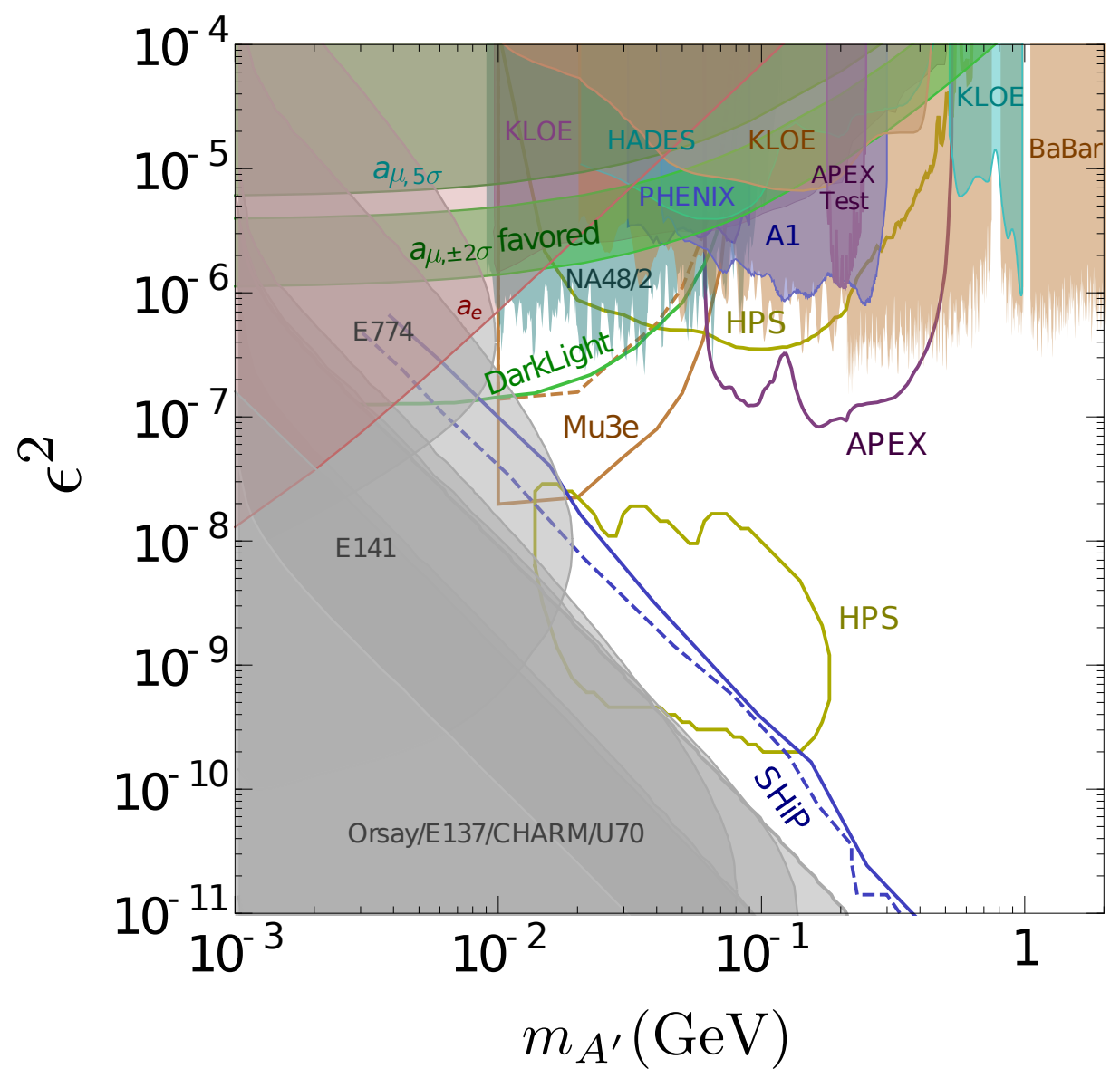

Figure 1.1: Parameter space for dark photons $\left(A^{\prime}\right)$ with mass $m_{A^{\prime}}>1 \mathrm{MeV}$. Here y-axis is $\epsilon^{2}=\alpha^{\prime} / \alpha$, where $\alpha=e^{2} / 4 \pi$, fine structure constant for ordinary electromagnetic interactions. $\alpha^{\prime}$ is the fine structure constant for dark matter. Cross-sections of $A^{\prime}$ production scale as $\epsilon^{2}$. This parameter space can be probed by different proposed experiments and hown are existing 90\% confidence level limits. The shaded areas indicate the results of already completed experiments and the lines indicate the area that will be covered by future experiments [6][16]. 
in Mainz are examples of fixed-target experiments that are searching for dark photons. BABAR [20] at SLAC and KLOE [21] at the DA $\Phi$ NE collider in Italy are examples for electron-positron collider experiments. A detailed description of other experimental procedures is discussed in Ref. [6].

\subsection{3 $A^{\prime}$ production in fixed target experiments}

An $A^{\prime}$ can be produced via electron beam scattering on a high-Z target such as Tungsten through a process similar to ordinary photon bremsstrahlung, and the cross section can be characterized as

$$
\sigma_{A^{\prime}} \sim 100 \mathrm{pb}\left(\frac{\epsilon}{10^{-4}}\right)^{2}\left(\frac{100 \mathrm{MeV}}{m_{A^{\prime}}}\right)^{2}
$$

where $m_{A^{\prime}}$ is the mass of the $A^{\prime}$ and $\epsilon^{2} \equiv \alpha^{\prime} / \alpha$ is the ratio of the effective fine structure constant for the $A^{\prime}$ and the fine structure constant $\alpha$ for ordinary electromagnetic interactions [7]. The cross section of equation 1.3 is several orders of magnitude higher than the expected $A^{\prime}$ production cross section in colliding electron and hadron beams [11].

The production of the $A^{\prime}$ boson can be reliably estimated using the WeizsäckerWilliams approximation [11]. For an incoming electron with energy $E_{0}$, the differential cross-section to produce an $A^{\prime}$ of mass $m_{A^{\prime}}$ with energy $E_{A^{\prime}}$ is,

$$
\frac{d \sigma}{d x d \cos \theta_{A^{\prime}}} \approx \frac{8 Z^{2} \alpha^{3} \epsilon^{2} E_{0}^{2} x}{U^{2}} \widetilde{\chi} \times\left[\left(1-x+\frac{x^{2}}{2}\right)-\frac{x(1-x) m_{A^{\prime}}^{2}\left(E_{0}^{2} x \theta_{A^{\prime}}^{2}\right)}{U^{2}}\right]
$$

where $x=E_{A^{\prime}} / E_{0}, \mathrm{Z}$ is the atomic number of the target atoms, $\alpha \simeq 1 / 137, \theta_{A^{\prime}}$, is 


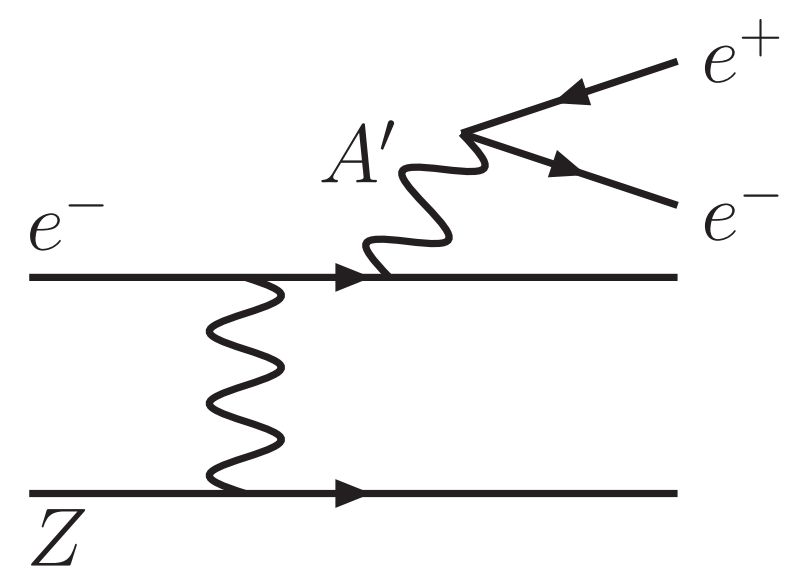

Figure 1.2: $A^{\prime}$ production by bremstrahlung off an incoming electron scattering off protons in a target with atomic number $\mathrm{Z}$. The $A^{\prime}$ particle could quickly decay into a $e^{+} e^{-}$pair. Figure taken from Ref. [31].

the angle in the lab frame between the emitted $A^{\prime}$ and the incoming electron.

$$
U\left(x, \theta_{A^{\prime}}\right)=E_{0}^{2} x \theta_{A^{\prime}}^{2}+m_{A^{\prime}}^{2} \frac{1-x}{x}+m_{e}^{2} x
$$

is the virtuality of the intermediate electron in initial-state bremsstrahlung, and $\tilde{\chi}$ $\equiv \chi / Z^{2} \sim 0.1-10$ is the Weizsäcker-Williams effective photon flux. This $\tilde{\chi}$ factor depends on kinematics, atomic screening, and nuclear size effects [11]. The above results are valid for,

$$
m_{e} \ll m_{A^{\prime}} \ll E_{0} \quad \text { and } \quad x \theta_{A^{\prime}}^{2} \ll 1
$$

Dropping $m_{e}$ and performing the angular integral, we obtain,

$$
\frac{d \sigma}{d x} \approx \frac{8 Z^{2} \alpha^{3} \epsilon^{2} x}{m_{A^{\prime}}^{2}}\left(1+\frac{x^{2}}{3(1-x)}\right) \tilde{\chi}
$$

The rate kinematics of $A^{\prime}$ radiation differ from massless bremsstrahlung in several important ways [11]. 
- Rate: For most $x, U(x, 0) \sim m_{A^{\prime}}^{2}$, so that the total production rate of $A^{\prime}$ depends on $\frac{\alpha^{3} \epsilon^{2}}{m_{A^{\prime}}^{2}}$. Therefore the $A^{\prime}$ production rate is suppressed relative to photon bremsstrahlung by $\sim \epsilon^{2} \frac{m_{e}^{2}}{m_{A^{\prime}}^{2}}$. An additional suppression from small $\tilde{\chi}$ occurs for large $m_{A^{\prime}}$ or small $E_{0}$.

- Angle: Most $A^{\prime}$ s are emitted at angles $\theta_{A^{\prime}}$ such that $U\left(x, \theta_{A^{\prime}}\right) \lesssim 2 U(x, 0)$ (beyond this point, wide angle emission falls as $1 / \theta_{A^{\prime}}^{4}$ ). For $x$ near its median value, the cut off emission angle is

$$
\theta_{A^{\prime} \max } \sim \max \left(\frac{\sqrt{m_{A^{\prime}} m_{e}}}{E_{0}}, \frac{m_{A^{\prime}}^{3 / 2}}{E_{0}^{3 / 2}}\right)
$$

which is smaller than the opening angle of $A^{\prime}$ decay products, $\sim m_{A^{\prime}} / E_{0}$.

- Energy: When $x \approx 1\left(E_{A^{\prime}} \approx E_{0}\right)$, where $U(x, 0)$ is minimized, $A^{\prime}$ production rate is sharply peaked. When an $A^{\prime}$ is produced, it carries nearly all of the beam energy.

After the $A^{\prime}$ is produced, it will travel for some distance before it decays into standard model particles. Its mean decay length which controls the vertex displacement (for the typical kinematics with $x \approx 1$ ) can be given by

$$
l_{0} \equiv \gamma c \tau \simeq \frac{3 E_{A^{\prime}}}{N_{\mathrm{eff}} m_{A^{\prime}}^{2} \alpha \epsilon^{2}} \simeq \frac{0.8 \mathrm{~cm}}{N_{\mathrm{eff}}}\left(\frac{E_{0}}{10 \mathrm{GeV}}\right)\left(\frac{10^{-4}}{\epsilon}\right)^{2}\left(\frac{100 \mathrm{MeV}}{m_{A^{\prime}}}\right)^{2}
$$

where $N_{\text {eff }}$ is the number of available decay products [27]]. For the ranges of $\epsilon$ and $m_{A^{\prime}}$ probed by this experiment, the mean decay length $l_{0} \lesssim 250 \mu \mathrm{m}$ is not significant. But the ability to reconstruct vertices displaced forward by a few centimeters allows us to probe even lower values of $\epsilon$. 
Using equation 1.7 , we can obtain an approximate expression for the rate of $A^{\prime}$ production. The number of $A^{\prime}$ s produced when a Coulomb of charge incident on a target with a thickness $T \ll X_{0}$ can be approximated by

$$
\frac{N_{A^{\prime}}}{C} \sim 10^{6}\left(\frac{T}{0.1}\right)\left(\frac{\epsilon}{10^{-4}}\right)^{2}\left(\frac{100 \mathrm{MeV}}{m_{A^{\prime}}}\right)^{2}
$$

where $C$ is the coulomb of charge and $X_{0}$ is the radiation length.

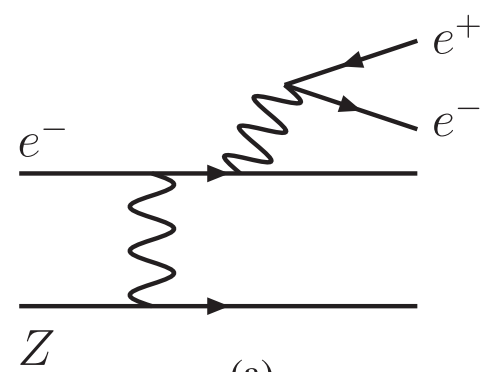

(a)

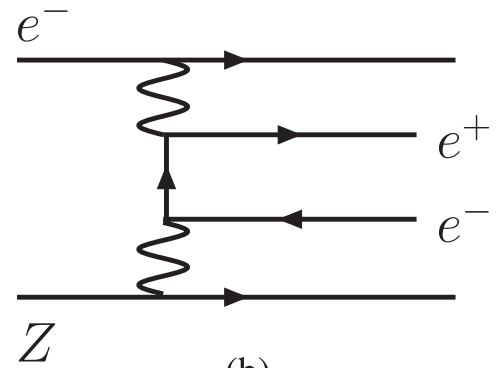

(b)

Figure 1.3: QED trident backgrounds. (a) radiative (b) Bethe-Heitler trident reactions that comprise the primary QED background to $A^{\prime} \rightarrow l^{+} l^{-}$.

Two main irreducible background rates during the $A^{\prime}$ experiment are shown in the figure 1.3. These background events, also known as QED trident events, can be separated into "radiative" diagrams (Figure 1.3 (a)) and "Bethe-Heitler" diagrams (Figure 1.3 (b)). The "Bethe-Heitler" process has a much larger cross-section than the other backgrounds, but, it can be significantly reduced by exploiting its very different kinematics [11]. The kinematics of the $A^{\prime}$ signal is identical to the distribution of "radiative" trident events. Therefore, it is useful to see the contribution of radiative events at different masses. The rate of the $A^{\prime}$ signal is simply related to the radiative trident cross-section within the spectrometer acceptance and a mass window of width 
$\delta m$ by

$$
\frac{d \sigma\left(e^{-} Z \rightarrow e^{-} Z\left(A^{\prime} \rightarrow l^{+} l^{-}\right)\right)}{d \sigma\left(e^{-} Z \rightarrow e^{-} Z\left(\gamma^{*} \rightarrow l^{+} l^{-}\right)\right)}=\left(\frac{3 \pi \epsilon^{2}}{2 N_{\mathrm{eff}} \alpha}\right)\left(\frac{m_{A^{\prime}}}{\delta m}\right) .
$$

Equation 1.11 summarizes the maximum achievable signal-to-background ratio that any experiment can achieve in an $A^{\prime} \rightarrow l^{+} l^{-}$search using only kinematics, with the decay vertex unresolved.

\subsection{APEX test run}

A test run for the APEX experiment took place in JLab Hall A in July 2010 [31]. A layout of the APEX test-run setup is shown in Figure 1.4. In the APEX test run, a $2.26 \mathrm{GeV}$ electron beam with an intensity upto $150 \mu \mathrm{A}$ was incident on a tantalum foil, with a thickness of $22 \mathrm{mg} / \mathrm{cm}^{2}$. Hall A High Resolution Spectrometers (HRS) were used to detect $e^{+} e^{-}$pairs. The left (right) HRS was positioned to accept electrons (positrons) with central momenta of $1.13 \mathrm{GeV} / \mathrm{c}$. The nominal angle of $e^{+} e^{-}$pairs produced as a result of a decay of an $A^{\prime}$ is $5^{\circ}$. However, the HRS's can only rotate to a minimum angle of $12.5^{\circ}$ with respect to the beamline. In order to detect $e^{+} e^{-}$pairs at small angles, a septum magnet was installed between the target and the spectrometers. The septum magnet was used to bend the $e^{+} e^{-}$pairs leaving the target at $5^{\circ}$ towards the HRS's. The septum magnet that was used for the APEX test run was not optimized for the APEX experiment. The sensitivity of the $A^{\prime}$ search depends critically on precise reconstruction of the invariant mass of $e^{+} e^{-}$pairs. The track reconstruction of the spectrometers was calibrated by placing a sieve plate, a $5 \mathrm{~mm}$ thick tungsten plate with a grid of holes, between the target and the entrance of the spectrometer. The track reconstruction can be optimized by 
comparing the surveyed and reconstructed hole positions and this method is known as sieve slit method [22]. Using APEX test run data, the mass range $175-250 \mathrm{MeV}$ was searched and found no evidence for an $A^{\prime} \rightarrow e^{+} e^{-}$reaction, and set an upper limit of $\alpha^{\prime} / \alpha \simeq 10^{-6}$.

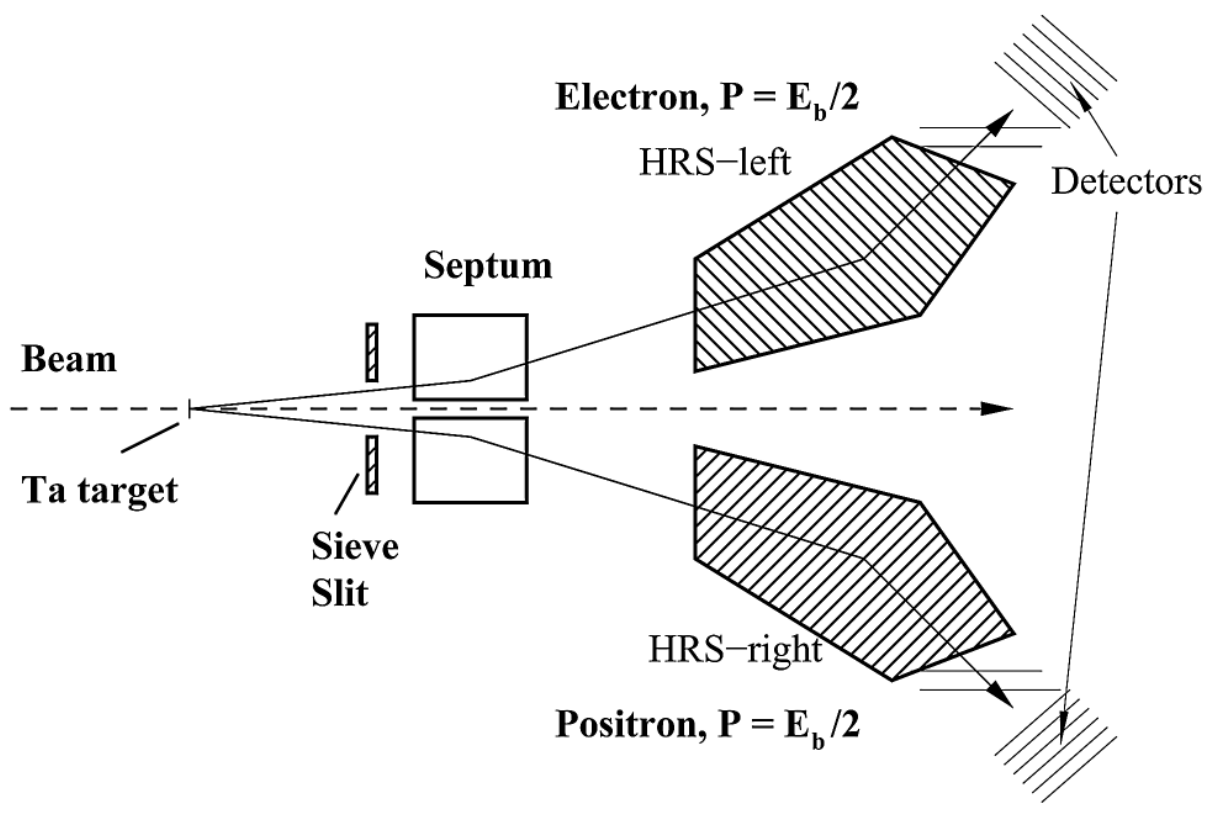

Figure 1.4: The layout of the APEX test run [31]. An electron beam was incident on a thin tantalum foil target. Two septum magnets of opposite polarity deflected charged particles towards HRS spectrometers. The HRSs contain detectors to accurately measure the momentum, direction, and identity of the particles. Sieve slit plates located in front of the septum magnets were used for calibration of the spectrometer magnetic optics.

\subsection{Brief overview of the approved JLab APEX experiment}

The $A^{\prime}$ experiment (APEX), which is approved to run at Thomas Jefferson Laboratory (JLab) will look for an $A^{\prime}$ particle in the mass range of $65 \mathrm{MeV}$ to $550 \mathrm{MeV}$ and 
$\epsilon^{2} \gtrsim 9 \times 10^{-8}$. The experiment will be performed in JLab Hall A using two highresolution spectrometers (HRS) [22]. An electron beam with an energy between $1 \mathrm{GeV}-4 \mathrm{GeV}$ and a current of $\sim 100 \mu \mathrm{A}$ will be incident on a $0.5 \%-5 \%$ radiationlength Tungsten target and the experiment will study the $e^{+} e^{-}$production in the target. The central angle for the spectrometers with a nominal target position is $5^{\circ}$. The electron will be detected in the left HRS and the positron will be detected in the right HRS. The $e^{+} e^{-}$pairs will be detected in coincidence within a timing window of $20 \mathrm{~ns}$. The rejection of pion backgrounds will be carried out by using the shower calorimeters and the gas Cherenkov counters. The invariant mass spectrum of $e^{+} e^{-}$ pairs will be scanned in the wide mass range of $65 \mathrm{MeV}$ to $550 \mathrm{MeV}$ for a narrow peak with a width corresponding to the instrumental resolution. This peak would correspond to the decay of an $A^{\prime}$ into an $e^{+} e^{-}$pair. A resonance search or "bumphunt" for a small signal peak over the continuous background will be performed and details will not be discussed in this thesis. The APEX experiment is planned to run with four kinematic settings. The primary run is planned to run using a setting with a beam energy of $2.2 \mathrm{GeV}$ for 6 days and setting with beam energy of $4.4 \mathrm{GeV}$ for 12 days. An additional run is proposed to use 6 days at $1.1 \mathrm{GeV}$ and $3.3 \mathrm{GeV}$. Further details about the APEX experiment will be discussed in Chapter 2. The APEX experiment plans to acquire $\sim 200$ times more data than the APEX test run.

\subsection{Structure of this thesis}

The Chapter 2 of this thesis is dedicated to discuss the APEX experiment, which is approved to run in Jefferson Lab Hall A. APEX is a high-luminosity experiment. Therefore, we expect a high rate of radiation which could potentially damage sensitive equipment in the JLab experimental hall A. A different high luminosity experiment, 
the "208 $\mathrm{Pb}$ Radius Experiment" (PREX-I), which ran in 2010 in JLab Hall used a $1.06 \mathrm{GeV}$ electron beam on a ${ }^{208} \mathrm{~Pb}$ target to perform parity violating measurements of neutron radii [32]. The PREX-I experiment had a significant loss of beam time due to vacuum degradation of the target region and a significant failure of HRS control system due to high radiation environments in the experimental hall [33][40]. Therefore, it is critical to perform detailed radiation analyses for future JLab experiments. Chapter 3 of this thesis discusses the radiation analysis carried out for the APEX experiment. The Monte Carlo simulation toolkit, GEANT4 (GEometry ANd Tracking version 4) was used to develop a detailed model of JLab Hall A and several experiments were simulated including APEX and PREX-I. Calculated radiation levels of the APEX experiment was compared with simulated and measured radiation levels of the PREX-I experiment. We found potential problems in neutron measurements in the experimental hall, and we discuss in great detail the potential sources of these problems and how to resolve them. This study will also help to better understand the radiation damage thresholds for the Hall A equipment.

APEX experiment will search for a small resonance peak on top of a continuous background. This requires a good mass resolution. In the APEX experiment, electrons will be detected in the left HRS and the positrons will be detected in the right HRS. The conventional HRS optics calibration technique, "Sieve-slit method", will not provide a good mass resolution in the positron arm. Therefore, two new Scintillating Fiber (SciFi) hodoscopes have been built at the JLab to perform a better HRS optics calibration. Chapter 4 presents results of a test that was done to calculate the efficiency of one of the SciFi detectors.

Neutron detectors need to be calibrated only using direct neutrons from the source. In calibration rooms it is unavoidable for the detectors to detect additional scattered neutrons. In Chapter 5, we study these scattering effects in a calibration room at JLab 
using a set of Bonner Spheres. A bonner-sphere set consists of several polyethylene spheres with different diameters with a thermal neutron detector at the center of the sphere. Different amounts of polyethylene makes each bonner sphere sensitive to different parts of a neutron spectrum, which then can be used to reconstruct an unknown neutron spectrum. Subsequently, a spectral subtraction method can be used to separate the scattered neutron spectrum. In this chapter we establish the ground work needed for a spectral reconstruction from bonner sphere measurements which included calculations of response functions of bonner spheres using Geant4 simulations. Chapter 6 presents summary of all the projects went into this thesis. 


\section{Chapter 2}

\section{The APEX Experiment}

The APEX experiment will study $e^{+} e^{-}$production due to an electron beam incident on a high-Z target as illustrated in Figure 2.1. The beam will pass through a target consisting of narrow strips of tungsten foil, with varying total thicknesses between $0.7 \% X_{0}$ and $5.3 \% X_{0}$ for each running configuration, where $X_{0}$ is the radiation length. The electron beam current will vary between $50 \mu \mathrm{A}$ and $120 \mu \mathrm{A}$ depending on the run setting and the beam will be rastered over an area either $1 \mathrm{~mm} \times 3 \mathrm{~mm}$ or $1.5 \mathrm{~mm} \times$ $5 \mathrm{~mm}$. The $e^{+} e^{-}$pairs leaving the target at a nominal angle of $5^{\circ}$ will be detected in the Hall A High Resolution Spectrometer (HRS). The detector package in each HRS includes two vertical drift chambers (VDC), a single-PMT trigger scintillator counter (S0), a Gas Cherenkov counter, a segmented high-resolution scintilator hodoscope, and a double-layer lead-glass shower counter.

\section{$2.1 \quad$ APEX target}

The APEX target ladder consists of 6 targets: an optics calibration target, an alignment target, 3 production targets and an alignment hole target. The APEX target is 


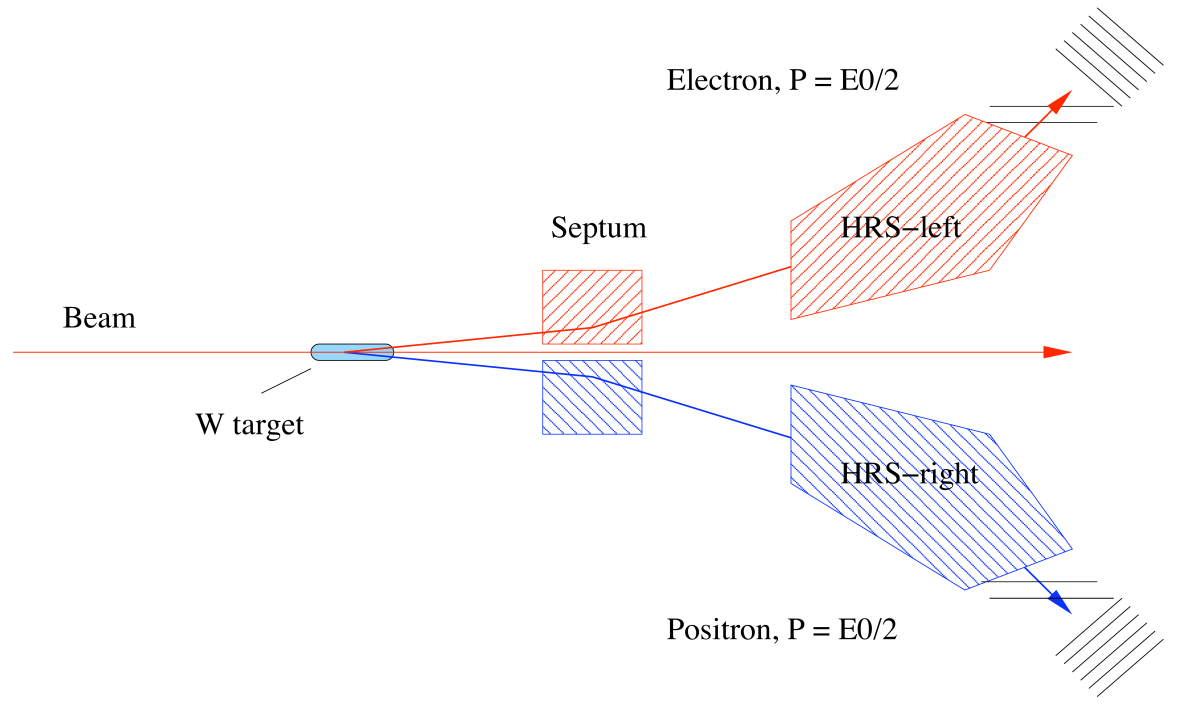

Figure 2.1: The layout of the experimental setup.

planned to be installed in the standard Hall A scattering chamber.

As indicated in Figure 2.2, the target at the bottom is for beam tuning. The second, third and fourth targets from the bottom are production targets. Each production target consists of up to 10 tungsten or tantalum ribbons, each held in an aluminium holder, specifically designed to keep the ribbon at tension and also to prevent distortions at high temperatures (see Figure 2.3). Each ribbon is $2.5 \mathrm{~mm}$ wide in order to accommodate raster of the beam. These $8 \mathrm{~mm}$ long foils are equally spaced along the beam line so that the distance between two adjacent ribbons is $5.5 \mathrm{~cm}$. With this spacing, outgoing $e^{+} e^{-}$pairs will completely miss the downstream ribbons. As a result, mass resolution is only limited by the thickness of a single ribbon, while the beam electrons pass through all 10 ribbons, thereby providing sufficient luminosity and minimal multiple-scattering effects. This elongated target also provides wide and uniform coverage in invariant mass of $e^{+} e^{-}$pairs. High-Z targets like tungsten and 

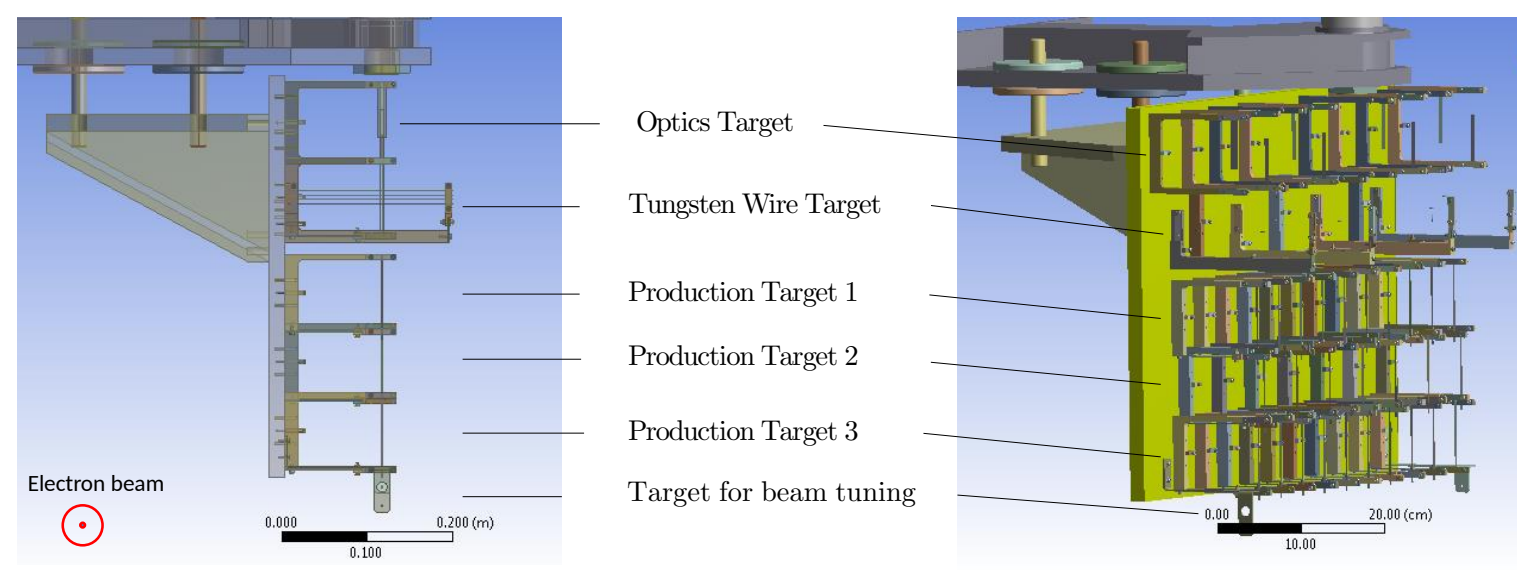

Figure 2.2: Overall layout of the APEX target system. The target ladder consists of six sections from top to bottom; an optics calibration target, an alignment target, 3 production targets and empty section for beam studies. Note that the target system is elongated along the beam line. The beam passes through all the production ribbons, while outgoing electron/positron pairs in the HRS acceptance only pass through one ribbon.

tantalum maximize the production rate of $e^{+} e^{-}$pairs as compared to pions, giving the highest possible signal to background ratio. This will also keep the background rate low enough, therefore, it will not overwhelm the trigger and data acquisition (DAQ) system.

As indicated in Figure 2.2, the second target from the top is the "Tungsten Wire Target" for beam-target alignment. This consists of 4 horizontal and 3 vertical tungsten wires, each $100 \mu \mathrm{m}$ in diameter. The wires are held by aluminum frames keeping the wires at tension in order to prevent sagging at high temperatures. Assuming the center of the target chamber is the origin, the 4 horizontal wires are located at $-25 \mathrm{~cm},-10 \mathrm{~cm}, 10 \mathrm{~cm}, 25 \mathrm{~cm}$ with a vertical step of $5 \mathrm{~mm}$ between each wire. In a similar manner, the 3 vertical wires are positioned at $-20 \mathrm{~cm}, 0 \mathrm{~cm}, 20 \mathrm{~cm}$ along the beam line with a horizontal step of $2.5 \mathrm{~mm}$. With the beam incident on the wires, beam-target alignment section will be used to precisely measure the beam direction 

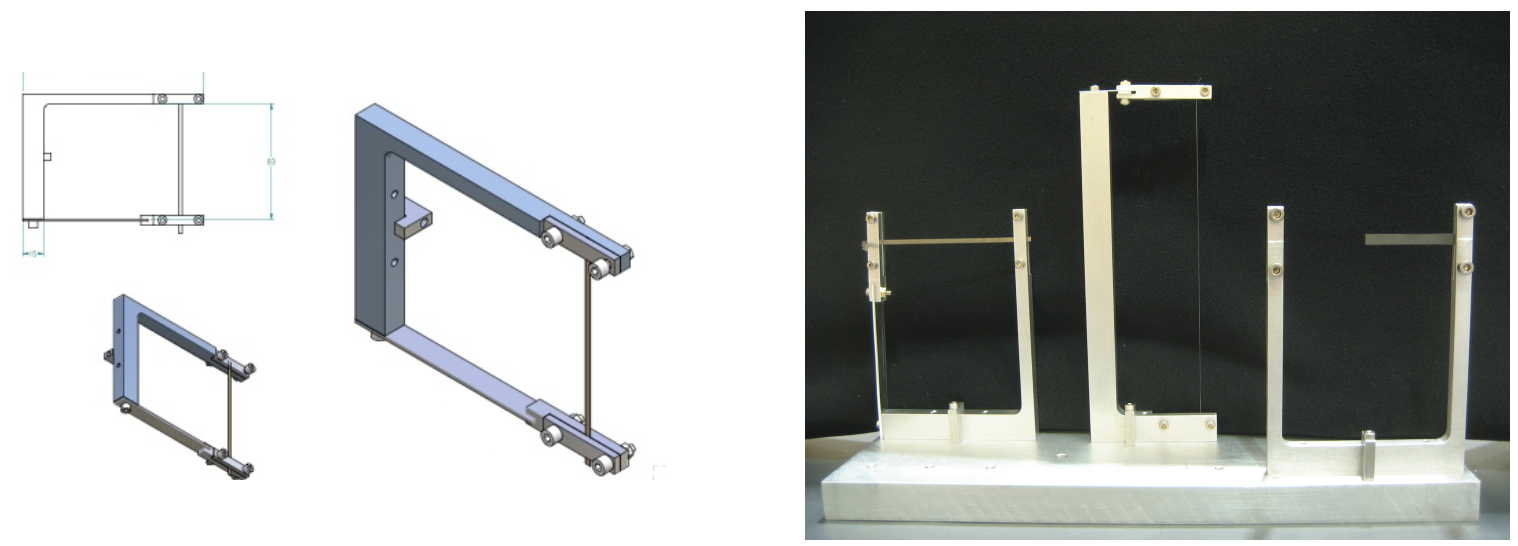

Figure 2.3: Left: Schematic illustration of the production ribbon holder. On one side of each holder is a tensioning bar that is spring loaded. This keeps the ribbons or wires straight when the material is hot. Right: A side view of production ribbon, beam-alignment wire and carbon optics holder.

relative to the alignment target.

As indicated in Figure 2.2, the target at the top of the target ladder is the optics calibration target. This consists of 8 carbon foils providing calibration points along the full length of the target system. The foils are arranged so that by changing the beam height, the beam can pass through either 4 foils or 8 foils. The electron beam can pass through 4 foils at a time, giving calibration with $14 \mathrm{~cm}$ spacing along the beam line, or 8 foils to provide an initial calibration with $7 \mathrm{~cm}$ spacing.

Table 2.1, summarizes the proposed run plan for the production targets. According to the thermal analysis, the maximum beam heating of an APEX target is $\sim 75 \mathrm{~W}$. That is when using a Tungsten target of $5.3 \%$ radiation length with an electron beam of $3.3 \mathrm{GeV}$ energy. Therefore, any external cooling mechanism is not required and all beam heating will be dispersed radiatively [34]. 
Table 2.1: Run plan for production targets. Total target thickness is given as a percentage of radiation length for each target material.

\begin{tabular}{|c|c|c|c|c|}
\hline $\begin{array}{c}\text { Energy } \\
(\mathrm{GeV})\end{array}$ & Target & $\begin{array}{c}\text { Beam Current } \\
(\mu A)\end{array}$ & Total Thickness & PAC days running \\
\hline 1.1 & $\mathrm{C}$ & 50 & $0.7 \%$ r.l. & 6 \\
\hline 2.2 & $\mathrm{~W} / \mathrm{Ta}$ & 100 & $2.8 \%$ r.l. & 6 \\
\hline 3.3 & $\mathrm{~W}$ & 120 & $5.3 \%$ r.l. & 6 \\
\hline 4.4 & $\mathrm{~W}$ & 90 & $5.3 \%$ r.l. & 12 \\
\hline
\end{tabular}

\section{$2.2 \quad$ APEX Septum Magnet}

The two HRS's can be rotated around the center of the experimental hall (shown as the pivot in Figure 2.4). In the APEX experiment, the nominal angle for the production of $e^{+} e^{-}$pairs is $5^{\circ}$ with respect to the beam line. But, the beam line and HRS components limit the rotation of the HRS spectrometers to $12.5^{\circ}$ with respect to the beam line. The ability to be sensitive to smaller scattering angles is achieved by moving the APEX target $105 \mathrm{~cm}$ upstream from the Hall A pivot point and placing a septum magnet at $70 \mathrm{~cm}$ downstream from the pivot. As illustrated in Figure 2.4, the septum magnet will bend the $e^{+} e^{-}$pairs leaving the target at $5^{\circ}$ towards the spectrometers which are positioned $12.5^{\circ}$ with respect to the beam line.

The APEX magnet has a length of $115 \mathrm{~cm}$ along the beam direction (z direction). It has a width (x direction) of $160 \mathrm{~cm}$ and a height (y direction) of $150 \mathrm{~cm}$. It is a water-cooled iron septum and it operates at $2100 \mathrm{~A}$, and $120 \mathrm{~V}$ for a field integral of 0.95 T-m. To reduce the fringe field effects, two corrector magnets will be used, one upstream of the septum magnet and the other one downstream.

Figure 2.5, shows a side view and a back view of the manufactured APEX septum magnet. Figure 2.6, shows a horizontal cross section of the final setup when septum 


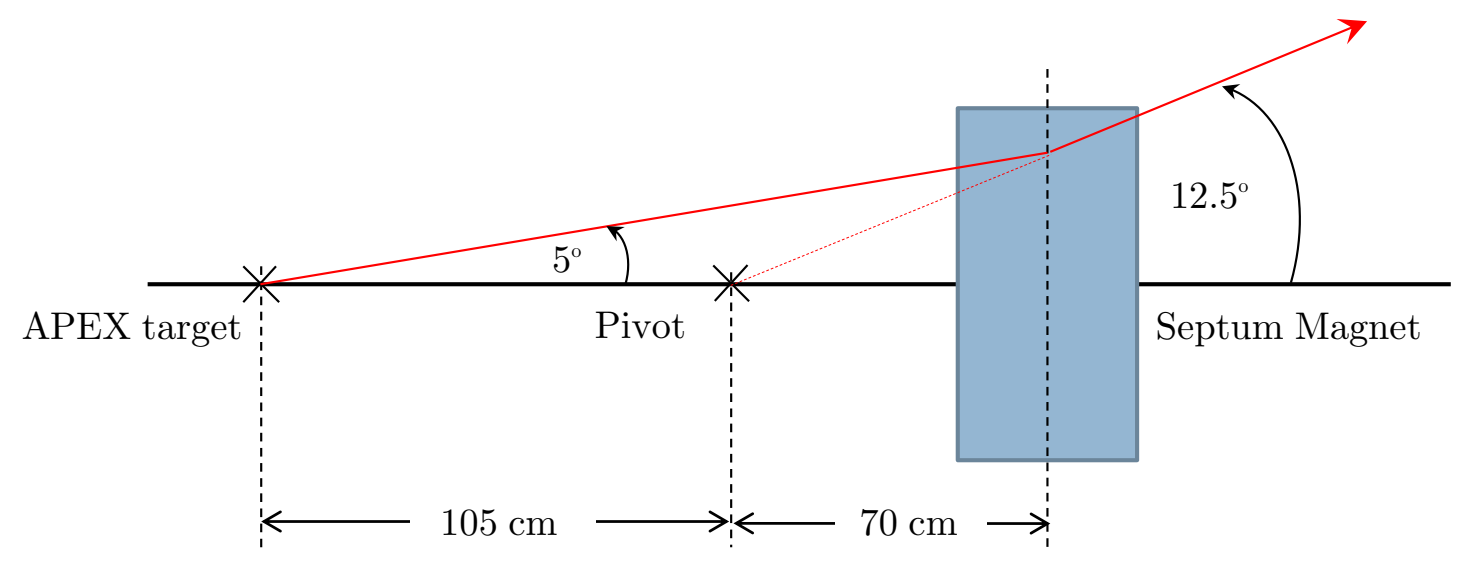

Figure 2.4: The sketch illustrates the top view of the APEX setup. An electron leaving the target at an angle of $5^{\circ}$ with the positive beam direction, is deflected by the septum magnet towards the left HRS positioned at $12.5^{\circ}$.

magnet is incorporated in to the beam line.

\subsection{The detector package}

Particles produced at the target pass through the septum magnet and then enter the HRS spectrometer acceptance. Each HRS consists of three quadrupole magnets (Q) and one dipole magnet (D) arranged in what is known as a QQDQ configuration. As shown in Figure 2.7, this configuration allows the charged particles to be bent vertically by $45^{\circ}$ angle with respect to the beam line [22]. The design characteristics of the HRS spectrometer is given in Table 2.2. The detector package of each HRS is located in a shielding hut at a height of $\sim 50$-feet above the ground.

The components of the two standard HRS detector packages in the JLab Hall A are shown in Figure 2.8. These detector packages are designed to perform various functions in the characterization of charged particles that pass into the spectrometer. A pair of vertical drift chambers (VDC) provide tracking information. The timing 

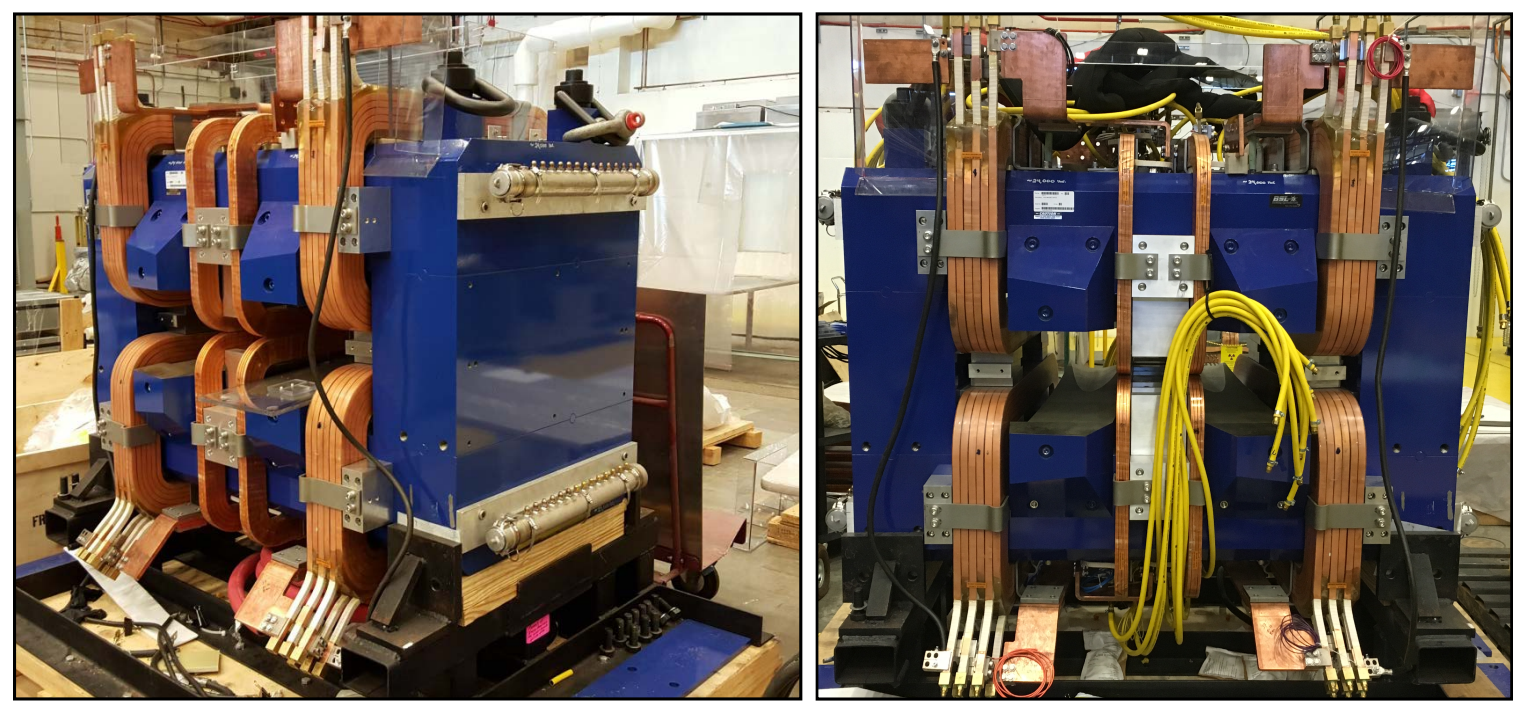

Figure 2.5: A side view (left) and a back view (right) of the fabricated septum magnet ready to be tested at JLab.

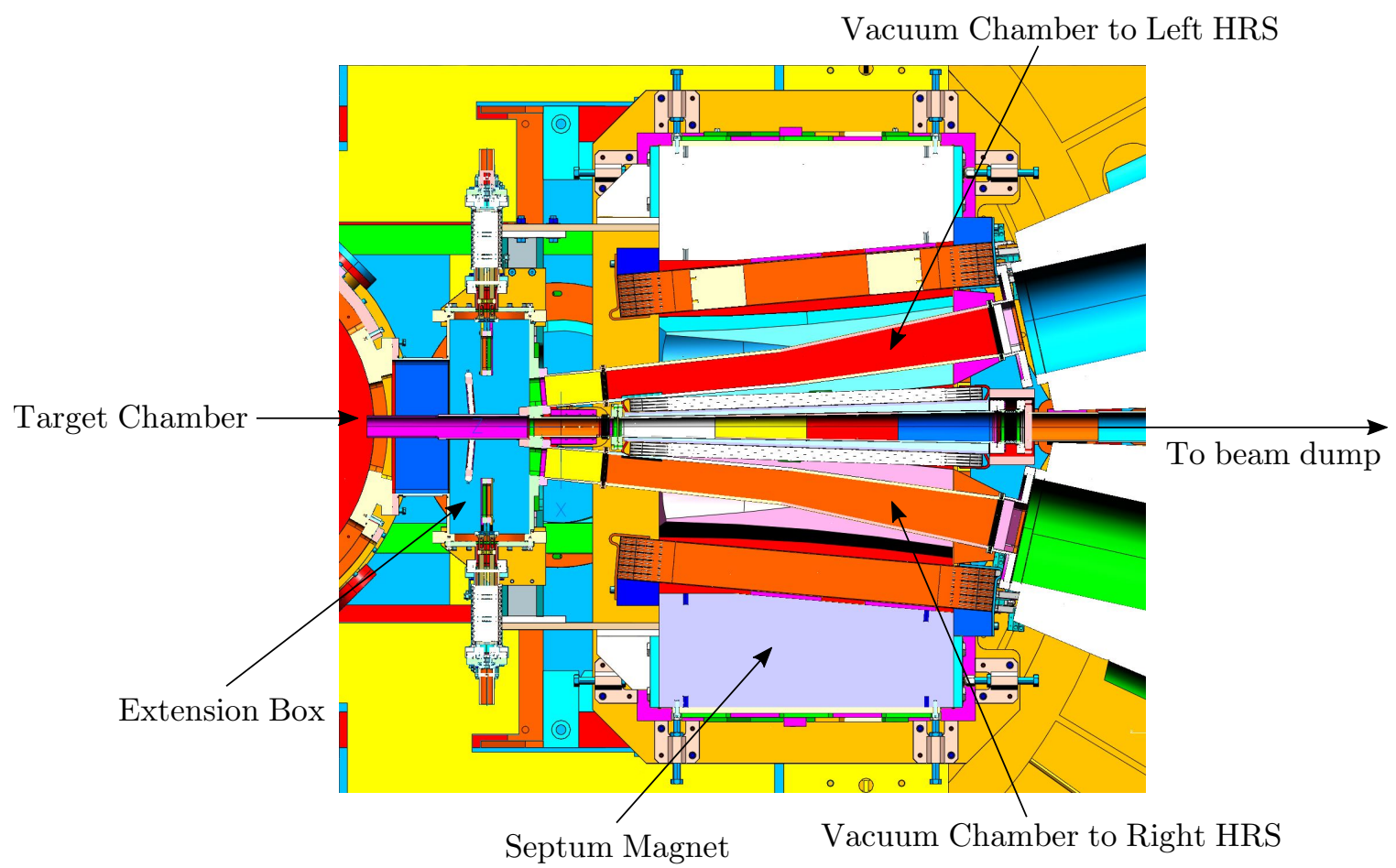

Figure 2.6: A horizontal cut of the beam line components around the septum magnet as viewed from the top. 
Table 2.2: Main design characteristics of the Hall A High Resolution Spectrometers at a nominal target position. The resolution values are for the FWHM.

\begin{tabular}{|l|c|}
\hline Configuration & QQD $_{\mathrm{n}} \mathrm{Q}$ Vertical bend \\
Bending angle & $45^{\circ}$ \\
Optical length & $23.4 \mathrm{~m}$ \\
Momentum range & $0.3-4.0 \mathrm{GeV} / \mathrm{c}$ \\
Momentum acceptance & $-4.5 \%<\mathrm{p} / \mathrm{p}<+4.5 \%$ \\
Momentum resolution & $1 \times 10^{-4}$ \\
Dispersion at the focus (D) & $12.4 \mathrm{~m}$ \\
Radial linear magnification $(\mathrm{M})$ & -2.5 \\
D/M & 5.0 \\
Angular acceptance & $\pm 30 \mathrm{mrad}$ \\
$\quad$ Horizontal & $\pm 60 \mathrm{mrad}$ \\
$\quad$ Vertical & $0.5 \mathrm{mrad}$ \\
Angular resolution & $1.0 \mathrm{mrad}$ \\
$\quad$ Horizontal & $6 \mathrm{msr}$ \\
Vertical & $\pm 5 \mathrm{~cm}$ \\
Solid angle at $\delta \mathrm{p} / \mathrm{p}=0, \mathrm{y}_{0}=0$ & $1 \mathrm{~mm}$ \\
Transverse length acceptance & \\
Transverse position resolution & \\
\hline
\end{tabular}


information is provided by scintillating layers what is shown in Figure 2.8 as the S0 and S2m layers. A Gas Cherenkov detector and two-layer lead-glass shower counters provide particle identification. The main parts of the detector packages in the two arms are identical except for the particle-identification detectors. The detector package and all DAQ electronics are located inside a shielding hut to protect against radiation backgrounds. More information on HRS detector packages can be found in Ref. [22].

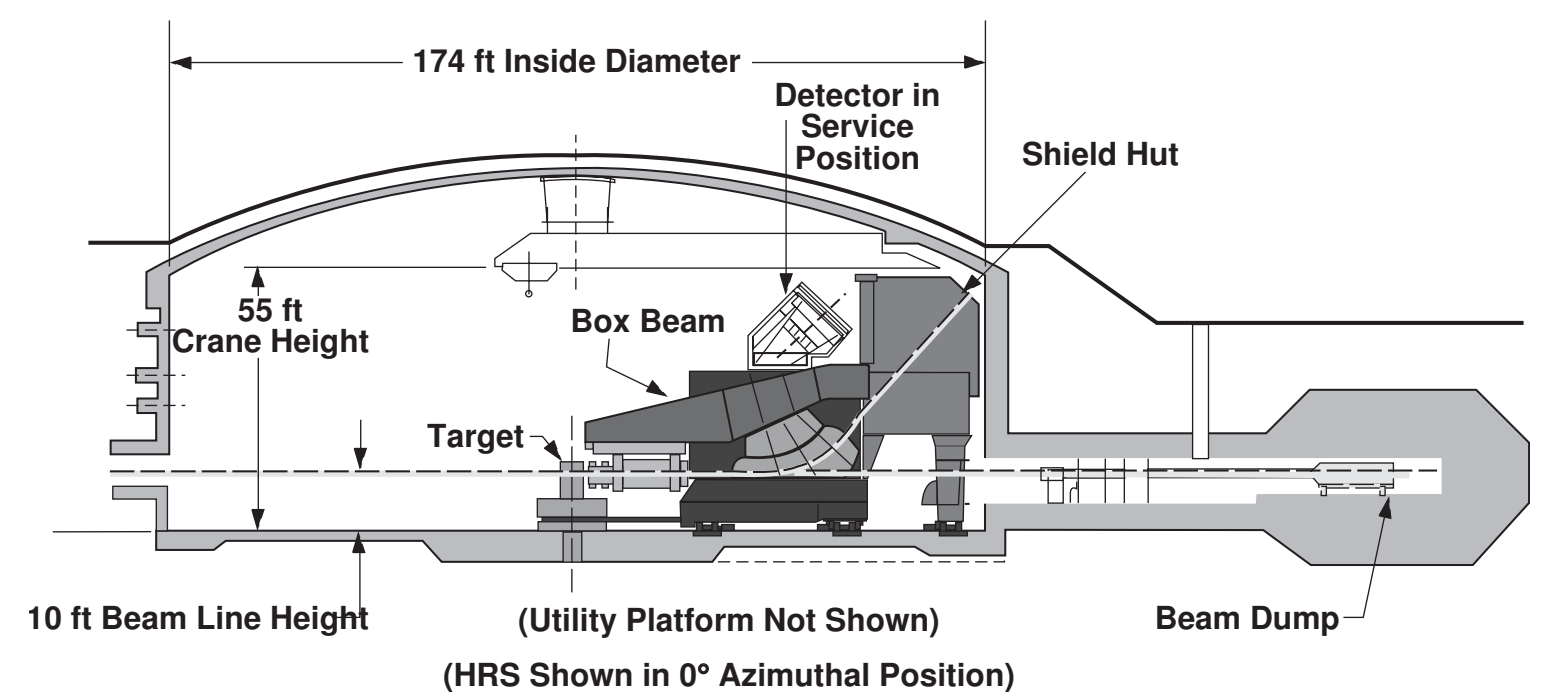

Figure 2.7: A schematic cross section of Hall A with one of the HRS spectrometers in the (fictitious) $0^{\circ}$ position. Figure from Ref. [22].

\subsection{Background Rates}

A search for a small signal always requires evaluation of the background. This section presents the background rates we expect during the APEX experiment. Table 2.3 summarizes the expected singles rates, trigger rates and coincidence rates.

The three main contributions to the counting rate in the spectrometers at small 

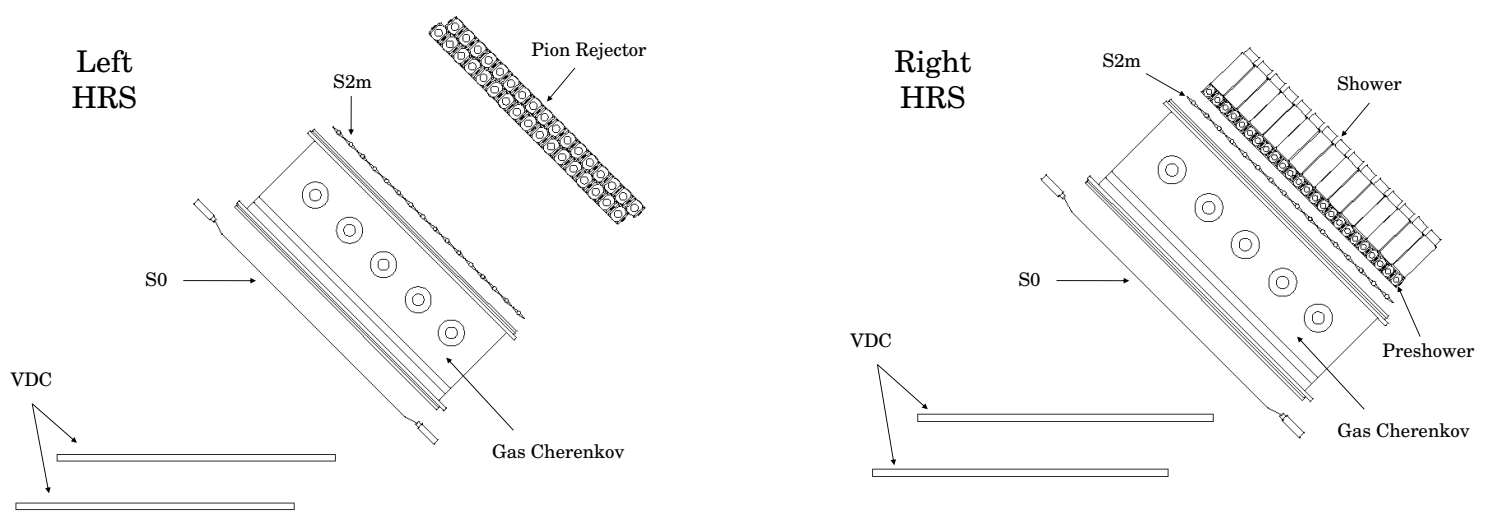

Figure 2.8: Side view of detector stack for the left and right HRS. Individual elements of the detector system are indicated in the confguration used for APEX. The position of the data-acquisition(DAQ) electronics and of the VDC support frame are not shown.

angles come from electrons, pions and protons which are scattered into the spectrometer acceptance. Electron singles rates in the spectrometers come from electrons produced in the processes of inelastic scattering, radiative elastic electron-nuclei scattering and radiative quasi-elastic electron-nucleon scattering. Known cross-sections of these processes have been employed to calculate these electron event rates [7]. The hadron production rates have been calculated elsewhere using the "Wiser" code [37], the EPC code [38] and the DINREG event generator [39]. The Wiser code has been widely used to estimate the pion background in electron scattering experiments at JLab. The Wiser code is based on parameterization of inclusive photo-production of protons, kaons, and pions [36]. The proton yield at the proposed kinematics will be $\sim 4-6$ times smaller than the pion yield. The calculated singles rates have also been checked against the data obtained during the APEX test run.

In the APEX experiment, the trigger is organized using a triple coincidence between trigger scintillator planes of two spectrometers and the Gas Cherenkov counter of the positron arm. More details about the trigger configuration will be discussed in 
the section 2.7. A relatively short coincidence time window of $20 \mathrm{~ns}$ will be used and this will help to keep the DAQ rate sufficiently low. The trigger rates shown in the Table 2.3 is calculated considering a $20 \mathrm{~ns}$ time window and a $\pi^{+}$rejection factor of 30 after including Gas Cherenkov in trigger. The trigger rate is about $3 \mathrm{kHz}$ for all APEX kinematic settings.

The HRS's are positioned to detect $e^{+} e^{-}$pairs as a result of the $A^{\prime} \rightarrow e^{+} e^{-}$ decay. Although the trigger is designed to only select correlated $e^{+} e^{-}$pairs (true coincidence events), there is still a significant amount of background events that is from the accidental coincidence events. Accidentals result from uncorrelated background events that happen to arrive at the detectors within the coincidence timing window. The accidental events will be a dominant part of the recorded events, which consist of $e^{+} e^{-}, \pi^{+} e^{-}, e^{+} \pi^{-}$and $\pi^{+} \pi^{-}$pairs. A series of offline analysis techniques will be used to reduce the background events. A 2 ns coincidence time window will be used to further suppress the accidental events. With the use of information from Gas Cherenkov and shower calorimeters an offline $\pi^{+}$rejection by a factor of 100 and a $\pi^{-}$rejection by a factor of 3 can be achieved. An additional factor of 4 rejection of accidentals can be achieved from the target vertex correlation between arms. With this selection, the irreducible background due to QED trident processes (shown in Figure 1.3) will dominate the total background. Table 2.3 summarizes the expected coincidence backgrounds during the APEX experiment.

\subsection{Particle identification}

There are two types of detectors installed in HRS's for the purpose of particle identification. They are a gas Cherenkov counter (GC) and a two-layer lead-glass calorimeter (LG). 
Table 2.3: Expected counting rates for APEX experiment. Settings A and B comprise the primary run plan, while setings $\mathrm{C}$ and $\mathrm{D}$ are additional settings at intermediate energies.

\begin{tabular}{|c|c|c|c|c|}
\hline Settings & A & B & C & $\mathbf{D}$ \\
\hline Beam energy $(\mathrm{GeV})$ & 2.2 & 4.4 & 1.1 & 3.3 \\
\hline Central angle & $5.0^{\circ}$ & $5.0^{\circ}$ & $5.0^{\circ}$ & $5.0^{\circ}$ \\
\hline Effective angles & $4.5^{\circ}-5.5^{\circ}$ & $4.5^{\circ}-5.5^{\circ}$ & $4.5^{\circ}-5.5^{\circ}$ & $4.5^{\circ}-5.5^{\circ}$ \\
\hline Target $T / X_{\mathrm{o}}$ & $4 \%$ & $8 \%$ & $0.7 \%$ & $8 \%$ \\
\hline Beam current $(\mu \mathrm{A})$ & 70 & 60 & 50 & 80 \\
\hline Central momentum (GeV) & 1.095 & 2.189 & 0.545 & 1.634 \\
\hline Singles (negative polarity) & & & & \\
\hline$e^{-}(\mathrm{MHz})$ & 4.1 & 0.7 & 4.5 & 2.2 \\
\hline$\pi^{-}(\mathrm{MHz})$ & 0.1 & 1.7 & 0.025 & 0.9 \\
\hline Singles (positive polarity) & & & & \\
\hline$e^{+}(\mathrm{kHz})$ & 27 & 5 & 18 & 17 \\
\hline$\pi^{+}[p](\mathrm{kHz})$ & 90 & 1700 & 25 & 900 \\
\hline Trigger/DAQ & & & & \\
\hline Trigger $(\mathrm{kHz})$ & 3.0 & 3.1 & 2.0 & 3.3 \\
\hline Coincidence Backgrounds & & & & \\
\hline Trident: $e^{-} Z \rightarrow e^{-} e^{+} e^{-} Z(\mathrm{~Hz})$ & 500 & 110 & 260 & 370 \\
\hline$e^{+} e^{-}$from real $\gamma$ conversion $(\mathrm{Hz})$ & 30 & 16 & 3 & 45 \\
\hline Accidentals $(\mathrm{Hz})$ & 55 & 30 & 40 & 40 \\
\hline
\end{tabular}


For the momentum range of this experiment, pions will not emit Cherenkov radiation. This makes the GC sufficiently blind to pions while being sensitive to both electrons and positrons. The detection probability of a pion in the GC of the HRS spectrometer is on the level of $1 \%$. As will be discussed in the next section, using the $\mathrm{GC}$ of the positron arm in the trigger configuration will reduce accidental coincidence events by a factor of 20-30. The LG has been used to analyze the particle identification efficiency of the GC. During the APEX test run, which ran in 2010, there was an additional run to test the high-rate performance of the HRS detectors. This additional test was performed using the lead/diamond target which was originally designed for the PREX-I experiment. It consisted of a $0.5 \mathrm{~mm}$ foil of lead sandwiched between two $0.2 \mathrm{~mm}$ sheets of diamond. The black line in Figure 2.9 (taken from Ref. [71]) shows the ADC sum of all PMTs in the Right-HRS GC for the lead/diamond target data. The narrow peak at the lower end of the spectrum (ADC amplitude $<100$ ) contains the meson background and the broad peak of events with higher $\mathrm{ADC}$ values (ADC amplitude $>100)$ contain the $e^{+}$signal. The blue and red lines correspond to $e^{+}$and meson background events as determined by the LG. Particles are identified in LG by plotting total energy in lead glass over momentum as shown in Figure 2.10. The first peak at the lower end of the spectrum contains $\pi^{+}$, the second peak contains $\mu^{+}$and the third peak contains $e^{+}$. The meson background efficiency was calculated by taking the fraction of meson background (as determined by the calorimeter) found in the GC $e^{+}$region. A similar approach has been used to calculate the $e^{+}$detection efficiency of the GC. When operating at a right-HRS trigger rate of $\sim 765 \mathrm{kHz}$, the Gas Cherenkov counter has a $99.2 \%$ detection efficiency of $e^{+}$events and a meson rejection efficiency of $97.0 \%$.

In high current tests during the APEX test run with positron rates comparable to expected rates for settings in the APEX full run, offline pion rejection of 1/30 (using 
the gas Cherenkov detector alone) and 1/60 (using the calorimeters alone) have been achieved. The combined rejection is more than the desired rejection of $1 / 100$ for positron arm. Since the electron/pion ratio in the left HRS is $1 / 3$ or higher, the pion rejection in the left HRS is not crucial.

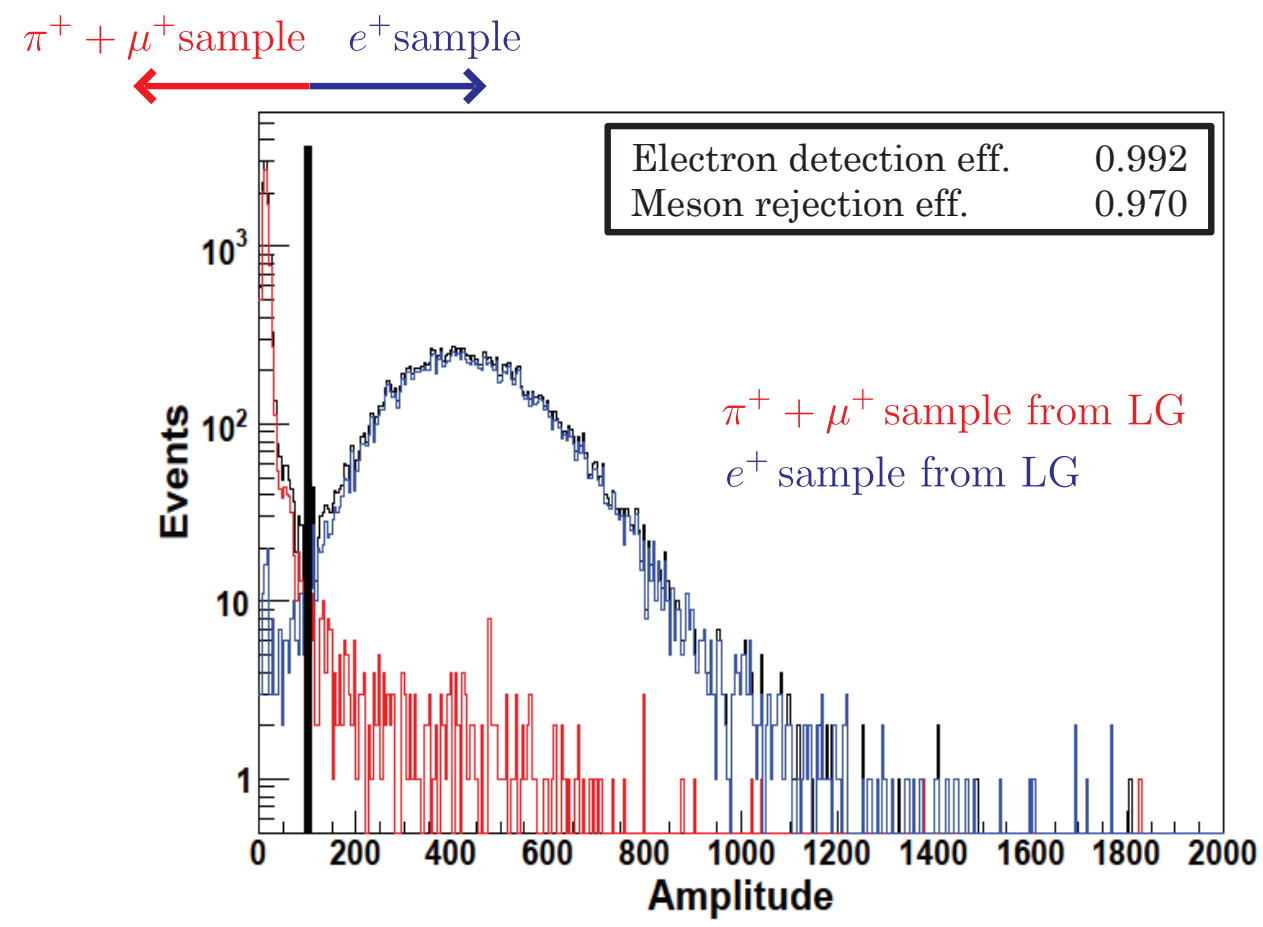

Figure 2.9: The particle identification with gas Cherenkov counter in the positron arm at the track rate of $765 \mathrm{kHz}$. The off-line pion rejection factor is 30 .

\subsection{Measurement of the particle track}

There are two vertical drift chambers (VDC) in the HRS detector package. VDC's will be used for the measurement of the position and the direction of tracks of particles arriving in the focal plane. Two VDC chambers are separated by $335 \mathrm{~mm}$. Each VDC 


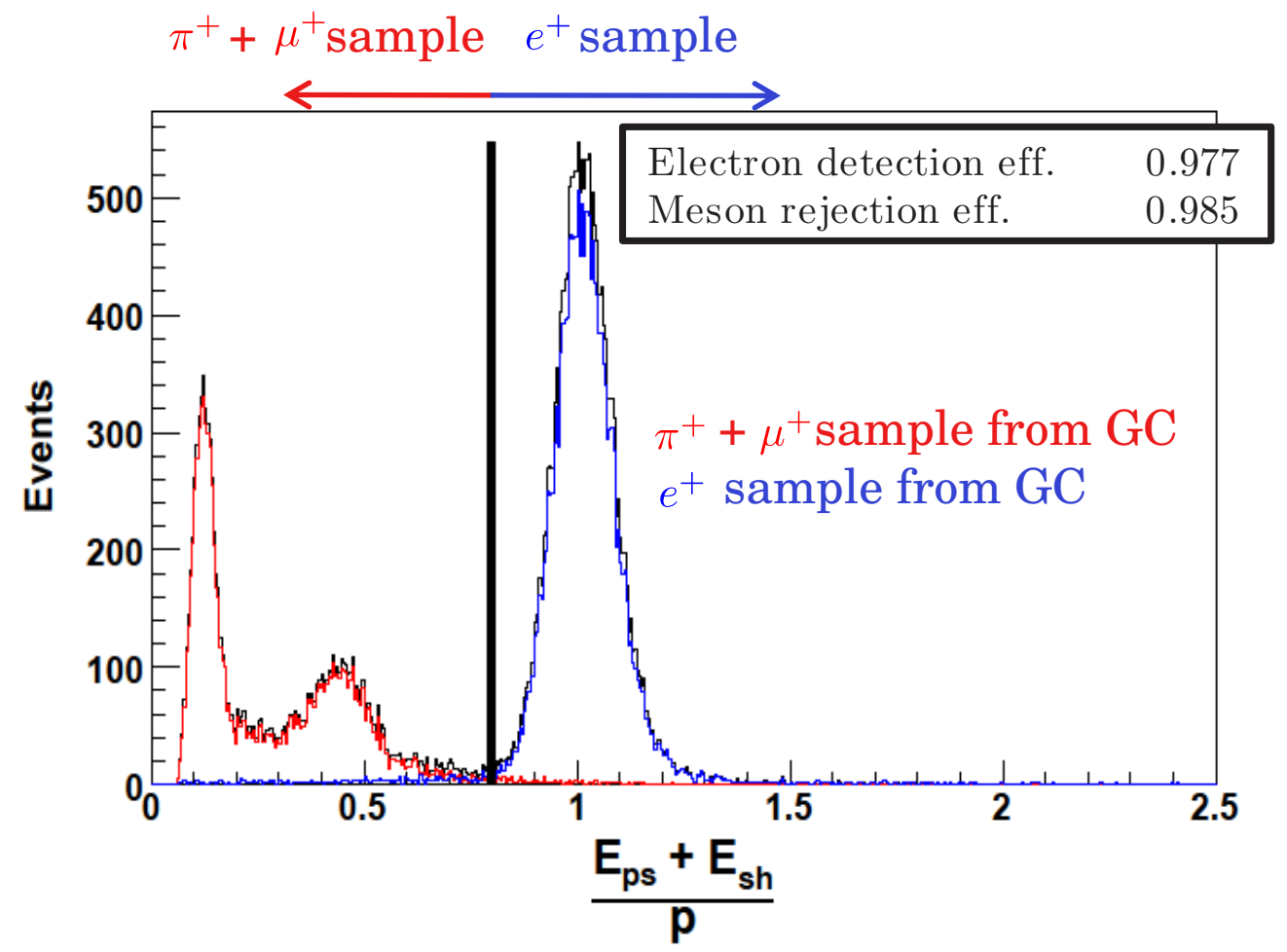

Figure 2.10: The total energy deposited in both layers pre-shower $\left(\mathrm{E}_{\mathrm{ps}}\right)$ and shower $\left(\mathrm{E}_{\mathrm{sh}}\right)$ of the calorimeter over the initial momentum of the incoming particles are shown. The first peak contains $\pi^{+}$events, the second contains $\mu^{+}$events, and the third contains $e^{+}$events. GC has been used to calculate the efficiency of the calorimeter. 
chamber has two wire-planes, separated by about $26 \mathrm{~mm}$. The active area of each wire plane is rectangular and has a size of $2118 \mathrm{~mm} \times 288 \mathrm{~mm}$. The wires of each plane are oriented at $90^{\circ}$ to one another. There are total of 368 wires in each " $U$ " and "V" direction, spaced $4.24 \mathrm{~mm}$ apart [22]. As shown in Figure 2.8, two VDC's are in the horizontal plane, so that the nominal particle trajectory crosses the wire planes at an angle of $45^{\circ}$. More information about the VDC's can be found in [43].

In the focal plane of the HRS, the VDC provides a position resolution of at least $0.2 \mathrm{~mm}$ and an angular resolution of $0.5 \mathrm{mrad}$. In some of the settings in APEX full run, the VDC in the left arm needs to be operated up to $\sim 5 \mathrm{MHz}$. During the APEX test run a $60 \%$ reconstruction efficiency at $5 \mathrm{MHz}$ track rate has been demonstrated.
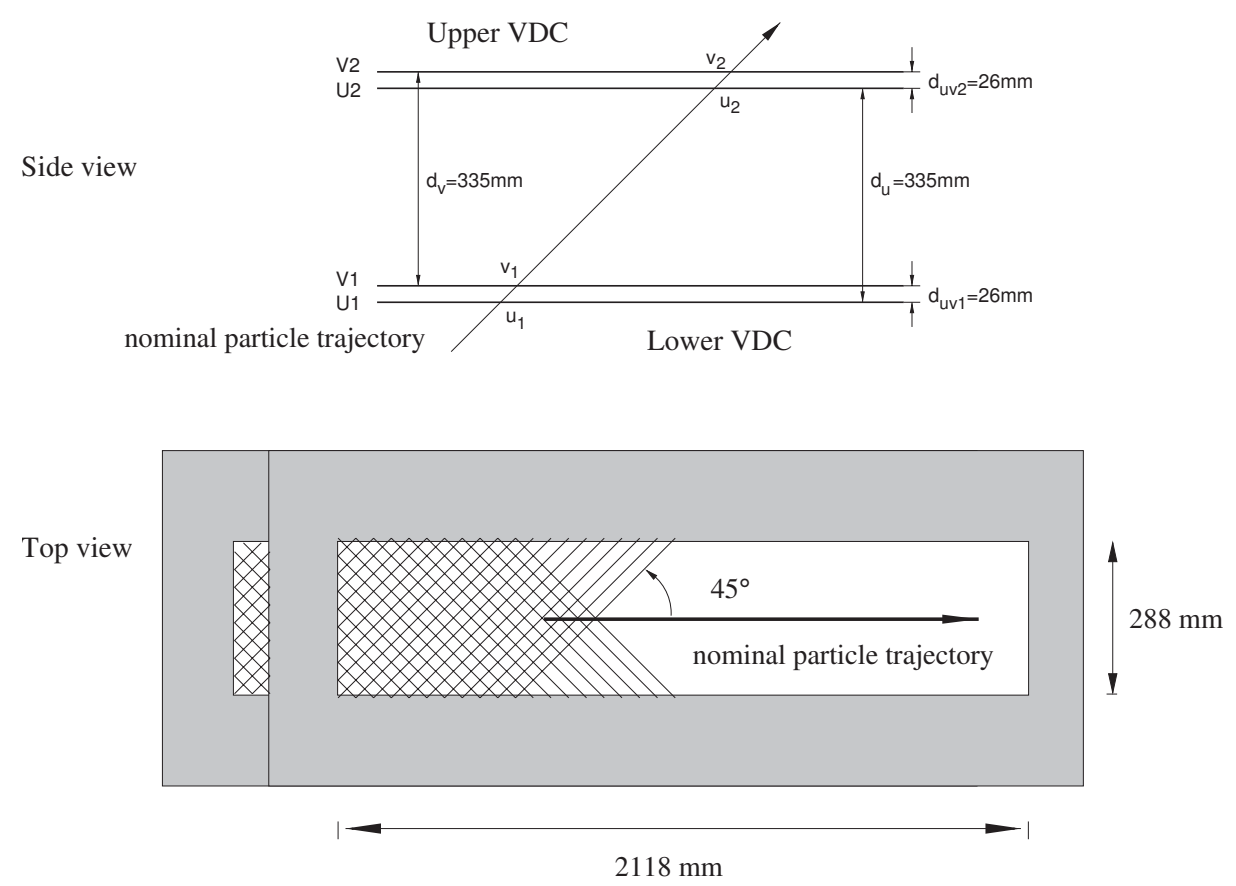

Figure 2.11: A schematic layout of Vertical Drift Chambers (VDC) for one HRS [22]. 


\section{7 $\quad$ Trigger and DAQ}

The trigger is the signal that makes the DAQ system start reading the detector outputs. Triggers can be configured to correspond to particles passing through single or multiple detectors. The trigger configuration is set up so that detector information is read out only for events of interest.

The APEX experiment is interested in detecting $e^{+} e^{-}$coincidence events. To select these events, the main trigger will consist of a coincidence between the $\mathrm{S} 2 \mathrm{~m}$ scintillator planes of both HRSs, and the gas Cherenkov counter of the right-HRS. This triple-coincidence including gas Cherenkov is necessary to reject pions in the event sample. Figure 2.12, illustrates a timing diagram of the trigger signal. When S2m scintillator plane in the left (right) HRS is hit by an electron (positron), it creates a $20 \mathrm{~ns}$ pulse. A $10 \mathrm{~ns}$ pulse will be formed when the gas Cherenkov detector in the right-HRS detects a signal. When all three pulses overlap, a trigger is formed, the data from all detectors is recorded and the event is considered as a "golden" coincidence event. The overlap of these three signals provides a coincidence timing window of $40 \mathrm{~ns}$.

In section 2.5 we discussed how GC was used to lower the meson background in the APEX test run data. Events with ADC amplitude less than 100 were considered meson background. The final data sample contained only a $0.9 \%$ meson background contamination and the accidental background has been estimated using the coincidence timing spectrum shown in Figure 2.13. Figure 2.13 illustrates an event distribution of the APEX test run data as a function of the time between the electron-arm $\mathrm{S} 2 \mathrm{~m}$ signal and the golden coincidence trigger. As indicated in the timing spectrum, the $10 \mathrm{~ns}$ wide peak contains the true coincidence events and the rest of the spectrum contains the accidental coincidence events. Based on the timing spectrum, the signal 
to background ratio of the APEX test run data has been calculated as $5 / 1$ and can be improved in the APEX full run. As can be seen in Figure 2.13, APEX test run used a 40 ns timing window. But, E. Jensen and S. Abrahamyan have shown that an even a shorter coincidence time window such as $20 \mathrm{~ns}$ can be easily achievable and this will keep the DAQ rate even lower [35]. The calculation of the expected event rates in the APEX experiment, as shown in Table 2.3, has considered a $20 \mathrm{~ns}$ timing window. With the use of the triple coincidence logic, the expected rate of the accidental events is at or below $3 \mathrm{kHz}$, which is well below the DAQ limit of $5 \mathrm{kHz}$.

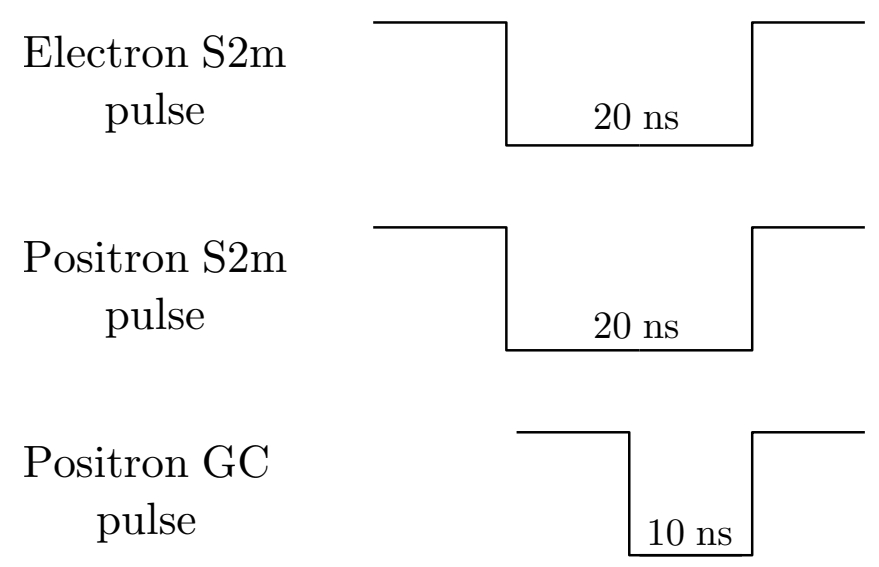

Figure 2.12: Timing diagram of the coincidence trigger. The overlap of both $20 \mathrm{~ns}$ S2m pulses and the $10 \mathrm{~ns}$ right-HRS gas Cherenkov pulse creates a "golden" coincidence trigger.

Analysis of the raw APEX data will yield an invariant mass spectrum similar to the one shown in Figure 2.14 taken from Ref. [31]. Figure 2.14 shows the invariant mass spectrum that was generated based on the APEX test run data. A resonance search or "bump-hunt" for a small signal peak over the continuous background needs to be performed. Further statistical analysis techniques that will be used to find the resonance peak will not be discussed here. More information on statistical methods 


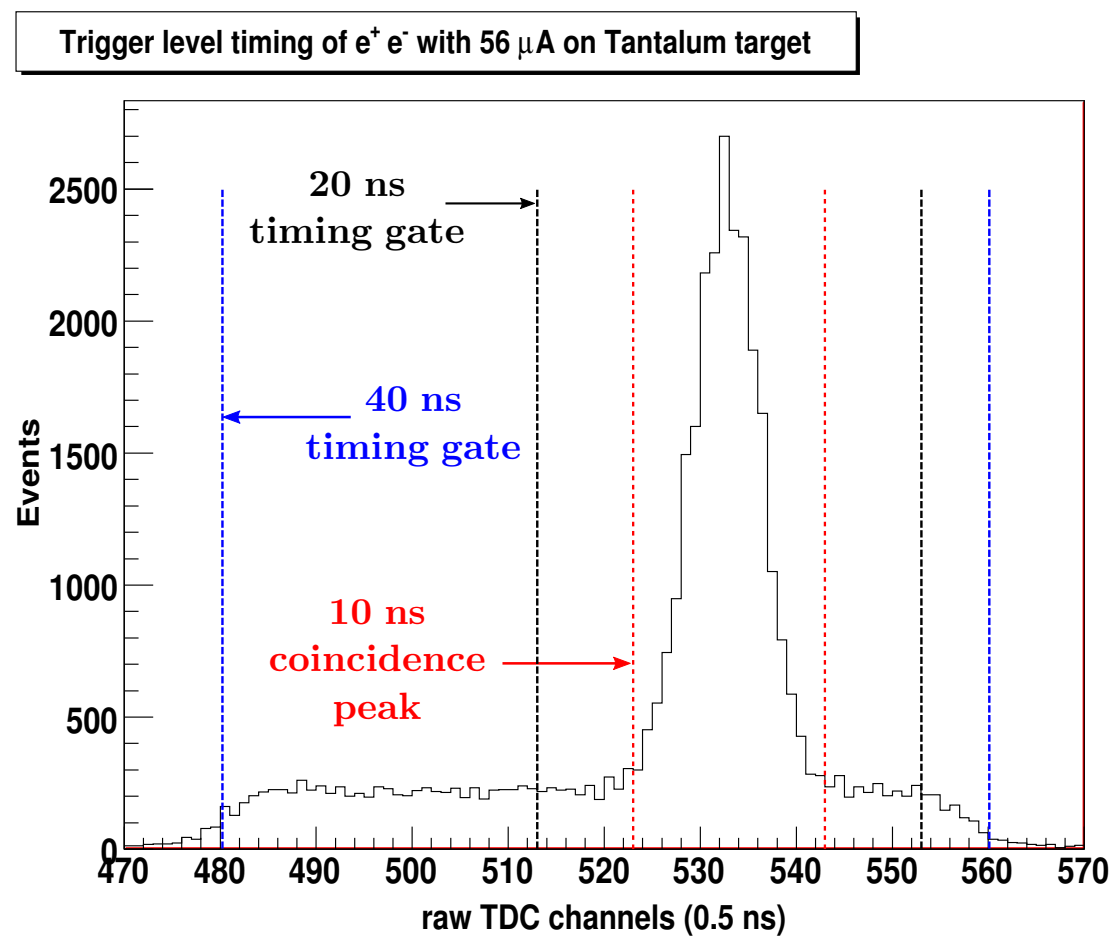

Figure 2.13: An event distribution of APEX test run vs. time between the electronarm S2m signal and the DAQ trigger signal. The total width of the accidental coincidence is $40 \mathrm{~ns}$. Even $20 \mathrm{~ns}$ trigger is possible. The real coincidence has a width of 10 ns. 


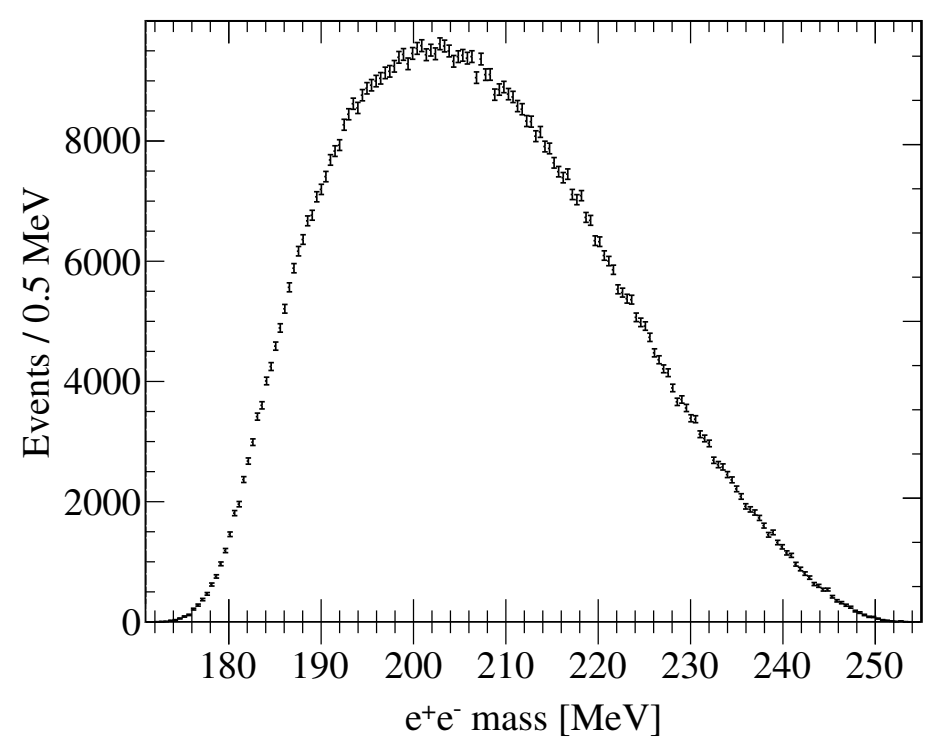

Figure 2.14: The invariant mass spectrum of $e^{+} e^{-}$pair events in the APEX test run. APEX test run searched for an $A^{\prime}$ particle in the mass range of $175-250 \mathrm{MeV}$. No hints for an $A^{\prime}$ was found in this mass window.

can be found in Ref. [31] [71]. APEX test run found no evidence of an $A^{\prime}$ signal. APEX experiment discussed in this chapter will probe a wider mass range and will have $\sim 100$ times higher statistics compared to the APEX test run. 


\section{Chapter 3}

\section{Monte-Carlo simulation of}

\section{radiation environments during}

\section{APEX}

The high luminosity in the $\mathrm{A}^{\prime}$ Experiment (APEX) will produce a lot of secondary particles in the beam line components, which could cause radiation damage in sensitive electronics, especially by neutron radiation. The addition of new beam line components during the APEX experiment makes the radiation studies even more important. The first " ${ }^{208} \mathrm{~Pb}$ Radius Experiment" (PREX-I), during its run, produced high background radiation inside Jefferson Laboratory (JLab) Hall A, which caused multiple failures in the control system of the High Resolution Spectrometer (HRS) [40]. Since then, JLab has been exercising extra precautions when it comes to highluminosity experiments. Here, we describe a GEANT4 [2] simulation that formed the basis for a review of radiation issues by the APEX readiness review committee.

GEANT4 (GEometry ANd Tracking) is a simulation toolkit, based on the C++ language, which can be used to simulate the passage of particles through matter. In 
this work, we developed a detailed model of JLab Hall A using Geant4 and simulated the APEX experiment. In addition to APEX, we simulated the PREX-I and RCS (Real Compton Scattering) experiments. We evaluated the radiation risk to the HRS control electronics and identified radiation sources in the beam line. Finally, as we will show in following sections, we found that the radiation risk expected during APEX is about 9 times lower than to the radiation risk that was present during PREX-I experiment.

\subsection{Geant4 configuration and a consistency check with previous codes}

We have developed a Geant4-based code for the exclusive purpose of radiation calculations relevant to APEX. Many other Geant4-based codes exist which incorporate aspects of the JLab Hall A geometry, but they focus on different issues. "G4SBS" is one such example which focuses on studying the performance of the Super-Bigbite Spectrometer. In my Geant4 code, I mainly focused on incorporating bulk material in the experimental hall which would be likely to produce secondary radiation. Development of the Geant4 model was done in a Dell Latitude E6540 laptop with an Intel Core i7-4600M Processor. Geant4 version 10.0.3 was installed on top of a Fedora linux operating system. Since the default build of Geant4 is sequential, multithreading was not used in this application. The high statistics simulations presented here were done in a high performance computer cluster called "Rivanna" at the University of Virginia. About $2 \times 10^{8}$ histories (each of which refers to a single particle) were used for each simulation.

For all the Geant4 simulations we performed, we used the "QGSP_BERT_HP" 
reference physics list [91] which is recommended for high-energy physics applications, especially which involve calculating neutron fluxes. The QGSP_BERT physics list includes a Geant4 standard package of electromagnetic physics [2][91]. This package provides simulation of electromagnetic interactions of photons, leptons, and hadrons in the energy range from $1 \mathrm{keV}$ to $10 \mathrm{PeV}$. In the QGSP_BERT physics list, final state generation is handled by two different physics models. It uses Bertini-style cascade (BERT) [90] for hadrons with energies $<10 \mathrm{GeV}$ and it uses a Quark-Gluon-String (QGSP) model for hadrons with energies $>20 \mathrm{GeV}$. QGSP_BERT_HP is the same as QGSP_BERT physics list, but with the addition of a high precision (HP) neutron model [91] used for neutrons $<20 \mathrm{MeV}$.

I performed two consistency checks, to be certain that the physics models included in my simulation are consistent with previous Monte-Carlo simulations. As the first consistency check, I compared the particle yield in my simulation, with a GEANT3 simulation developed by Pavel Degtiarenko of the in Radiation Control Department at JLab. We simulated the particle yield from an $11 \mathrm{GeV}$ electron beam incident on a carbon target with a thickness of $0.193 \mathrm{~cm}$ (1\% radiation length) and a diameter of $5 \mathrm{~cm}$. Figure 3.1 illustrates the comparison of particle yield calculated using Geant 4 and Geant3 simulations. Plots on the left, $a(1), b(1)$ and $c(1)$, were generated using Geant3 calculations and plots on the right, $\mathrm{a}(2), \mathrm{b}(2)$ and $\mathrm{c}(2)$, were generated using the Geant4 code developed for this study. Particle yield calculations using Geant3 and Geant 4 were in reasonable agreement. For instance, the number of photons with $10 \mathrm{MeV}$ of energy leaving the target at an angle between $1.0^{\circ}$ and $10.0^{\circ}$ is roughly $1 \times 10^{-5}$ electron ${ }^{-1} \mathrm{MeV}^{-1} \mathrm{sr}^{-1}$, in both Geant3 and Geant4 calculations. As illustrated in plots $\mathrm{b}(1)$ and $\mathrm{b}(2)$ in Figure 3.1, the number of electrons produced in the target and leaving at an angle between $10.0^{\circ}$ and $45.0^{\circ}$ with an energy of $40 \mathrm{MeV}$ is roughly $1 \times 10^{-6}$ electron ${ }^{-1} \mathrm{MeV}^{-1} \mathrm{sr}^{-1}$ in both Geant3 and Geant4 calculations. The 
electron production at angles between $0.0^{\circ}$ and $1.0^{\circ}$ is not directly comparable. In plot $\mathrm{b}(1)$, the sharp peak at $10^{4} \mathrm{MeV}$ is due to the primary electron beam. In the Geant4 calculation (plot b(2) in Figure 3.1), electrons from the primary beam were not recorded to reduce the size of the data file. Plots c(1) and c(2) in Figure 3.1 illustrate the neutron yield, with low statistics. Neutrons leaving at angles between $10.0^{\circ}$ and $45.0^{\circ}$ had reasonable statistics and had a yield of $1 \times 10^{-6}$ electron ${ }^{-1} \mathrm{MeV}^{-1} \mathrm{sr}^{-1}$ of $1 \mathrm{MeV}$ neutrons in both cases.

A second consistency check was done by comparing the particle fluence calculated at a certain location in JLab Hall A for the PREX-I setup. The PREX collaboration also developed a simulation code based on Geant 4 for radiation studies. At the time of this work, the Geant4 simulation model developed by the PREX collaboration lacked a model of the HRS spectrometers. Therefore, I simplified my Geant4 model of Hall A to match their model to perform a direct comparison. Figure 3.2 illustrates the JLab Hall A geometry and the PREX-I setup taken from the two Geant4 simulations. In Figure 3.2, a(1) and a(2) show an isometric view and a top view, taken from the Geant4 simulation developed by Rakitha Beminiwattha for the PREX collaboration. On the other hand, $b(1)$ and $b(2)$ in the same figure show an isometric and a top view of the Geant4 model developed for this study. In both simulations, an electron beam of $1.06 \mathrm{GeV}$ energy was incident on a $0.5 \mathrm{~mm}$ (10\% radiation length) thick Lead target. In Monte-Carlo simulations, a geometric volume is often identified from which information can be gathered regarding the passage of particles. This is referred to as "tallying" or "scoring". The geometric volumes used to score particles are often called "scoring-volumes" or "sensitive-volumes". A Geant4 scoring-volume with dimensions of $2 \mathrm{~m} \times 2 \mathrm{~m} \times 2 \mathrm{~m}$ was placed $\sim 20 \mathrm{~m}$ downstream of the target. The chosen material of the scoring-volume was gas with a very low density, $1 \times 10^{-25} \mathrm{~g} / \mathrm{cm}^{3}$, which is essentially the Geant 4 implementation of vacuum. The particle fluence can 

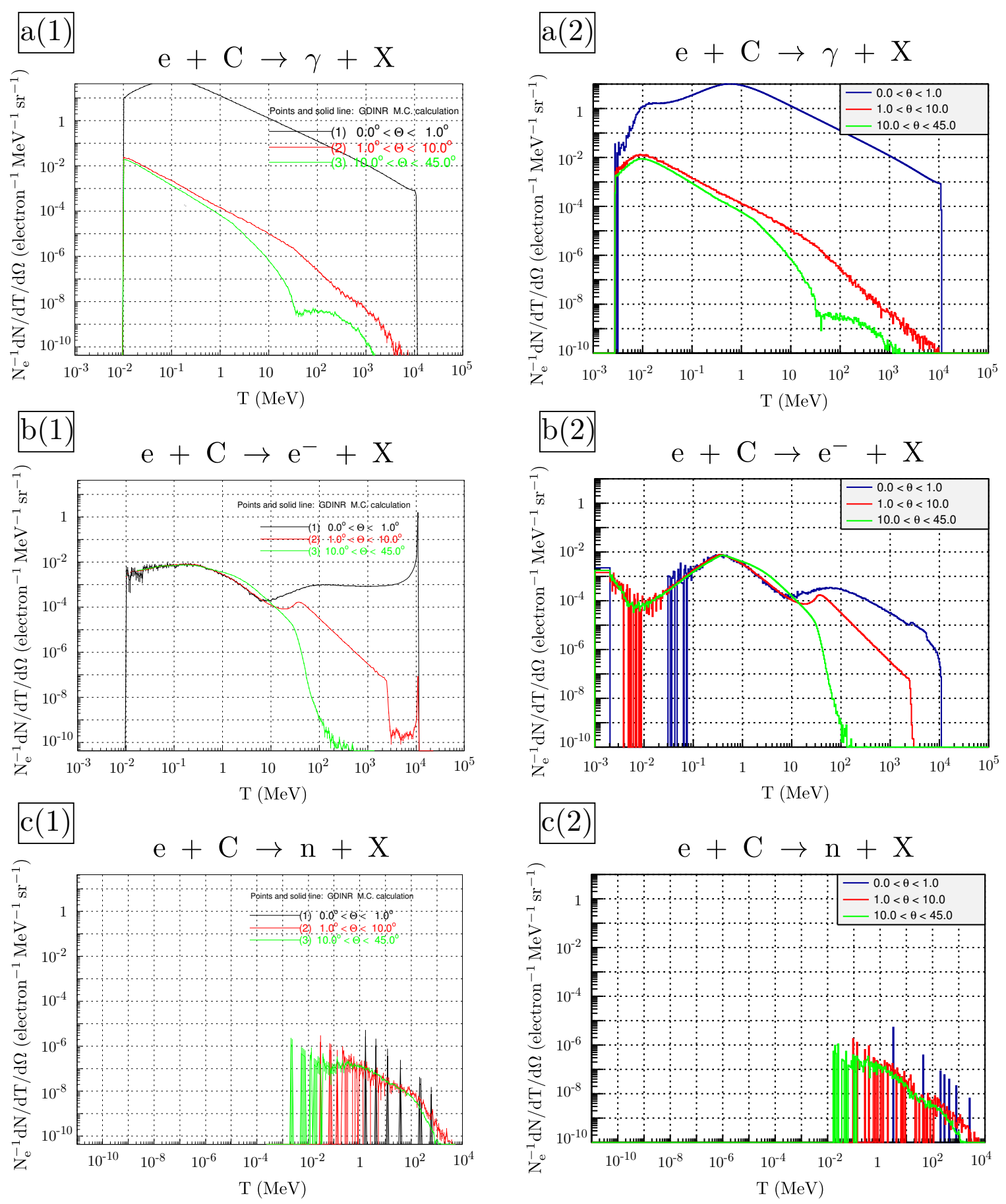

Figure 3.1: Plots illustrate photon, electron and neutron production from $11 \mathrm{GeV}$ electron beam incident on a $1 \%$ r.l. Carbon target. Plots a(1), b(1) and c(1) were generated using a GEANT3 simulation and $\mathrm{a}(2), \mathrm{b}(2)$ and $\mathrm{c}(2)$ were generated using GEANT4 simulation developed for this study. In all plots, y-axis indicates the number of events per $\mathrm{MeV}$ and per unit sold angle normalized by number of incident electrons. $\mathrm{X}$-axis is the kinetic energy of particles leaving the target. Plots with different colors separate particles by its opening angle $\theta$ with respect to beam direction. 
be calculated using two methods. The first method is to record all the particles that enter the scoring-volume from all directions and divide by the total outside area of the scoring-volume. I refer to this method as the "area-method". In dosimetric calculations, it is recommended to use a different method to calculate the particle fluence. In this second method of calculating particle fluence, the total track length of particles within the scoring-volume was divided by the volume of it [92]. I refer to this method as "volume-method". I will further discuss the volume method and how I calculated particle fluence in a later section (see section 3.3.1). In the simulations performed by the PREX collaboration, they used the area-method to calculate particle fluence. Therefore, only for this comparison, I adopted the area-method. For later calculations, I used the volume-method. Table 3.1 summarizes the particle fluence calculated by both the PREX collaboration and in what is presented here. The photon fluences calculated using the two Geant4 models agreed within 30\% and electron fluences agreed within $20 \%$. Neutron fluences with energies lower than $10 \mathrm{MeV}$ agreed within $1 \%$ which is probably fortuitous. Fluence of high energy neutrons (above $10 \mathrm{MeV}$ ), calculated using my Geant4 code were a factor of 1.6 higher than those calculated by the PREX collaboration. Given the nature of calculations, factor of 1.6 seems fully reasonable.

\subsection{Geant4 model}

\subsubsection{Hall A}

Figure 3.3 illustrates the Geant4 model of JLab Hall A constructed for this simulation. JLab hall $\mathrm{A}$ is a cylindrically shaped building with a radius of $\sim 27 \mathrm{~m}$. The concrete walls are about $16 \mathrm{~m}$ high and they are about $40 \mathrm{~cm}$ thick. The floor is concrete, $2 \mathrm{~m}$ 

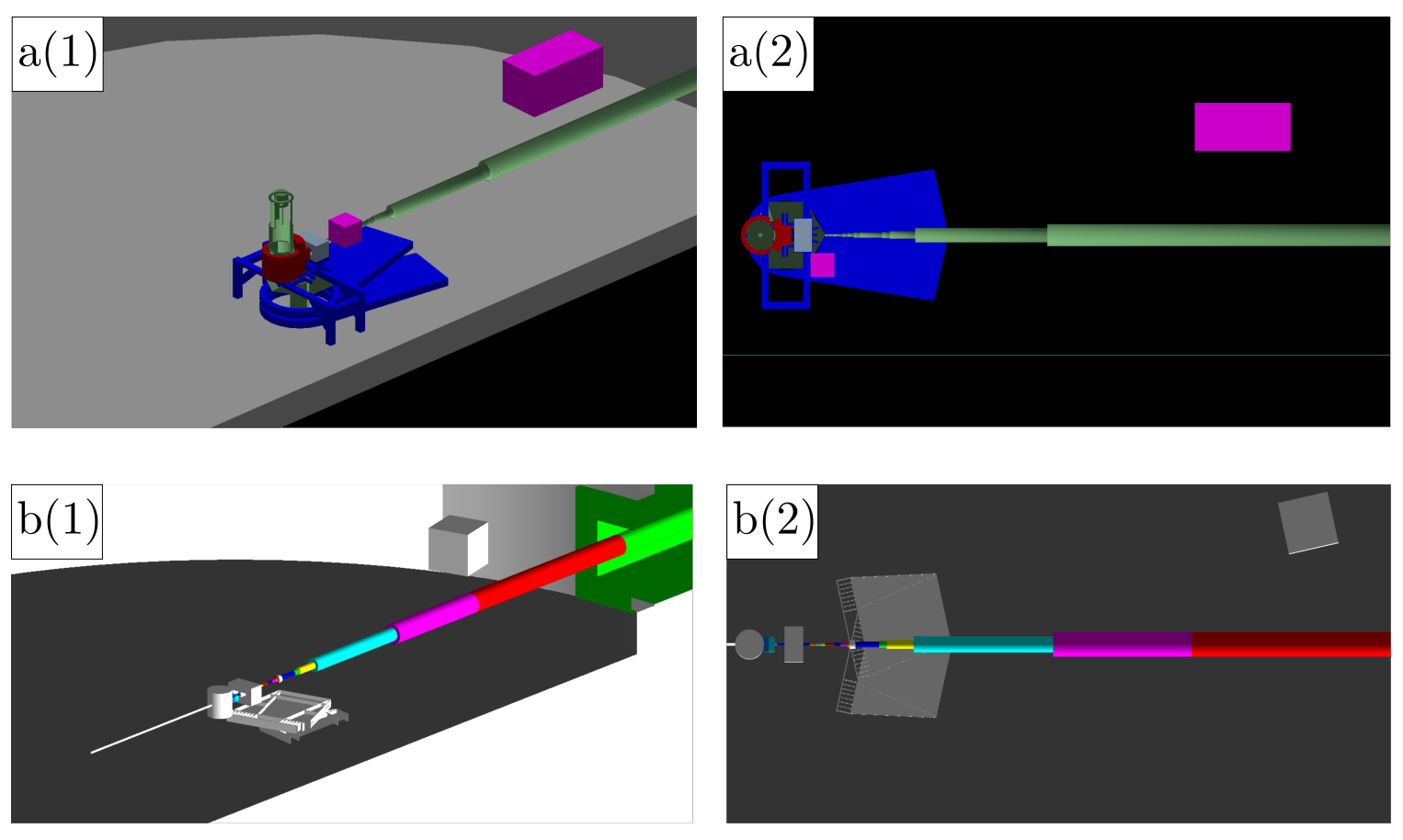

Figure 3.2: Geant4 model of hall A used by PREX collaboration for their simulations [89] 
Table 3.1: Comparison of simulated particle fluence of PREX-I experiment using a Geant 4 model developed by the PREX collaboration and the model developed for this work. The area-method, described in the text, was used to calculate the particle fluence in both cases.

\begin{tabular}{|c|c|c|c|}
\hline Particle type & $\begin{array}{c}\text { Energy range } \\
(\mathrm{MeV})\end{array}$ & $\begin{array}{c}\text { This work: } \\
\text { particle } \\
\text { fluence } \\
\left(\mu A^{-1} m^{-2}\right)\end{array}$ & $\begin{array}{c}\text { PREX } \\
\text { collaboration: } \\
\text { particle } \\
\text { fluence } \\
\left(\mu A^{-1} m^{-2}\right)\end{array}$ \\
\hline$\gamma$ & $E<10$ & $4.6 \mathrm{E}+09$ & $2.99 \mathrm{E}+09$ \\
& $E>10$ & $2.2 \mathrm{E}+08$ & $1.64 \mathrm{E}+08$ \\
\hline$e^{-} / e^{+}$ & $E<10$ & $2.0 \mathrm{E}+08$ & $1.87 \mathrm{E}+08$ \\
\hline$n$ & $E>10$ & $2.1 \mathrm{E}+08$ & $1.75 \mathrm{E}+08$ \\
\hline & $E<10$ & $1.2 \mathrm{E}+07$ & $1.19 \mathrm{E}+07$ \\
& $E>10$ & $1.9 \mathrm{E}+05$ & $3.12 \mathrm{E}+05$ \\
\hline
\end{tabular}


thick, and the roof is a dome with concrete $50 \mathrm{~cm}$ thick. This entire structure is below ground level except the dome. The dome is covered with a thin layer of soil with a thickness of $\sim 65 \mathrm{~cm}$. The downstream beam pipe starts from roughly the center of the hall (as viewed above) and goes through a tunnel with thick concrete walls and ends at a beam dump which is located about $30 \mathrm{~m}$ past the edge of the experimental hall. All these dimensions were taken from the engineering drawings and simplified to include in the model.
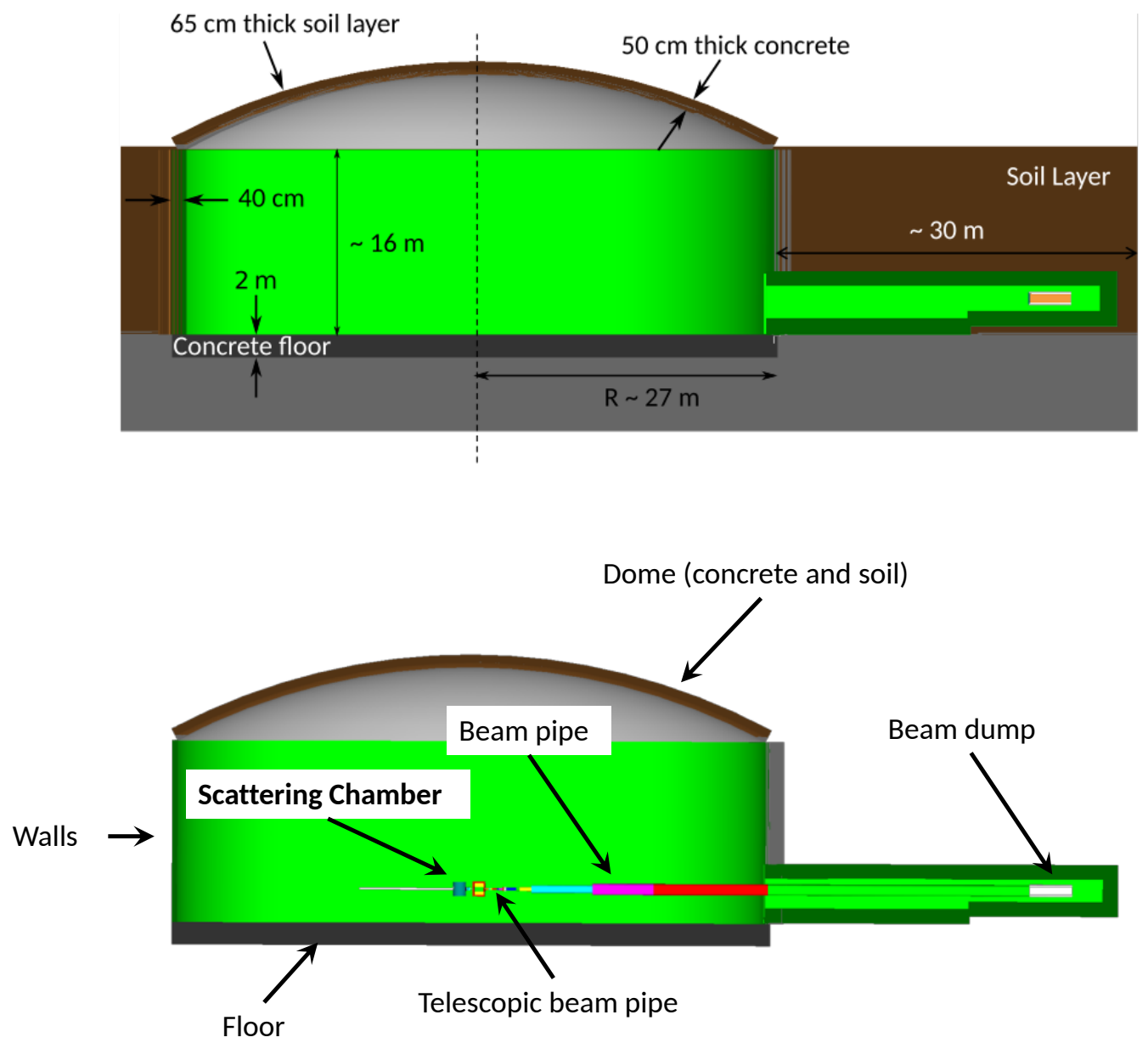

Figure 3.3: This figure illustrates the Geant4 model of JLab hall A. Top and Bottom images show the same geometry with different features labeled. 


\subsubsection{Hall A Beamline}

The Geant4 model of JLab Hall A developed for this work includes the downstream beamline. We followed engineering drawings very closely when describing the beam pipe, as it can be a main source of secondary radiation. Figure 3.4 and Table 3.2 summarize details of the beamline used within the Geant 4 model. The pipes labeled 1 - 3 are made of regular aluminum and the inside of the pipe is filled with low density air. The pipes labeled as $4-8$ are made of corrugated steel. But, in the Geant4 model we used regular pipes instead of corrugated pipes. To include the effects of corrugation, a low density material was used, so that the weight per unit length of pipes we used in Geant4 match with that of corrugated pipes. The beamline ends at a beam dump consisting of a water barrel.

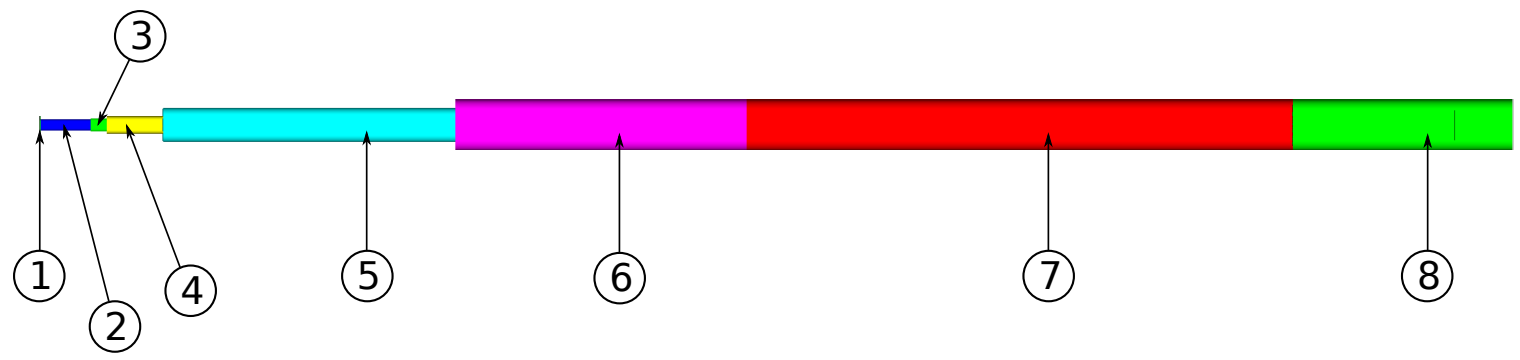

Figure 3.4: Geant4 model of the downstream beamline in JLab hall A. Different colors are used to indicate different sections of the beamline. The numbered labels correspond to the raws in the Table 3.2.

\subsubsection{Components in the High Resolution Spectrometer (HRS)}

Figure 3.5 shows a side view of one of the HRS spectrometers taken from engineering drawings. Some parts were not included in the Geant4 model for simplicity and parts that were included are highlighted and labeled in Figure 3.5.

Figure 3.6 and Figure 3.7 illustrate a front view and a side view of the Geant4 
Table 3.2: Inner diameter, outer diameter and length of different sections of the downstream beam line are listed here.

\begin{tabular}{|c|c|c|c|c|}
\hline & $\begin{array}{c}\text { ID } \\
\text { (inches) }\end{array}$ & $\begin{array}{c}\text { OD } \\
\text { (inches) }\end{array}$ & $\begin{array}{c}\text { Length } \\
\text { (inches) }\end{array}$ & $\begin{array}{c}\text { Material density } \\
\left(\mathbf{g} / \mathbf{c m}^{3} \text { ) }\right.\end{array}$ \\
\hline 1 & 8.02 & 13.5 & 0.75 & 2.699 \\
\hline 2 & 8.0 & 8.25 & 36.875 & 2.699 \\
\hline 3 & 9.75 & 10.0 & 12.0 & 2.699 \\
\hline 4 & 12.0 & 13.0 & 41.8125 & 0.3761 \\
\hline 5 & 24.0 & 25.0 & 217.0 & 0.3718 \\
\hline 6 & 36.0 & 38.0 & 216.375 & 0.2124 \\
\hline 7 & 36.0 & 38.0 & 405.1875 & 0.2124 \\
\hline 8 & 36.0 & 38.0 & 120.4375 & 0.2124 \\
\hline
\end{tabular}

model of the HRS respectively. The two hall A HRS's use a Quadrupole-QuadrupoleDipole-Quadrupole (QQDQ) optics design. Quadropole magnets were simplified by a hollow iron cylinder. The coils of the quadropole magnets were not included in the model. Similarly, the dipole magnet was described using an iron core. The magnetic fields were not included in either the quadrople or dipole magnets. The multi-layered structure (iron, lead and concrete) in the actual detector hut (shield hut) to shield the detector package from radiation, was also included in the simulation model. In the Hall A HRS spectrometer, all three quadrupoles and the drift chamber detector elements are hung from a steel structure called the "box beam" [22]. The front part of the box beam has a thick concrete structure referred to as the "line-of-sight" shielding. This line-of-sight shielding, which was also included in the Geant4 model, blocks muons created in the decay of pions in flight from reaching detector packages installed in the detector hut. At the floor level, each HRS is composed of three sections: a cradle, a HRS gantry and a link. The gantry holds the detector hut in place. The cradle holds the entire structure and it can rotate around the hall center to accommodate different scattering angles. Both the cradle and the gantry are made 
out of steel plates and are hollow inside. When describing these elements we were careful to include all the bulk material which might be responsible for the production of particles.

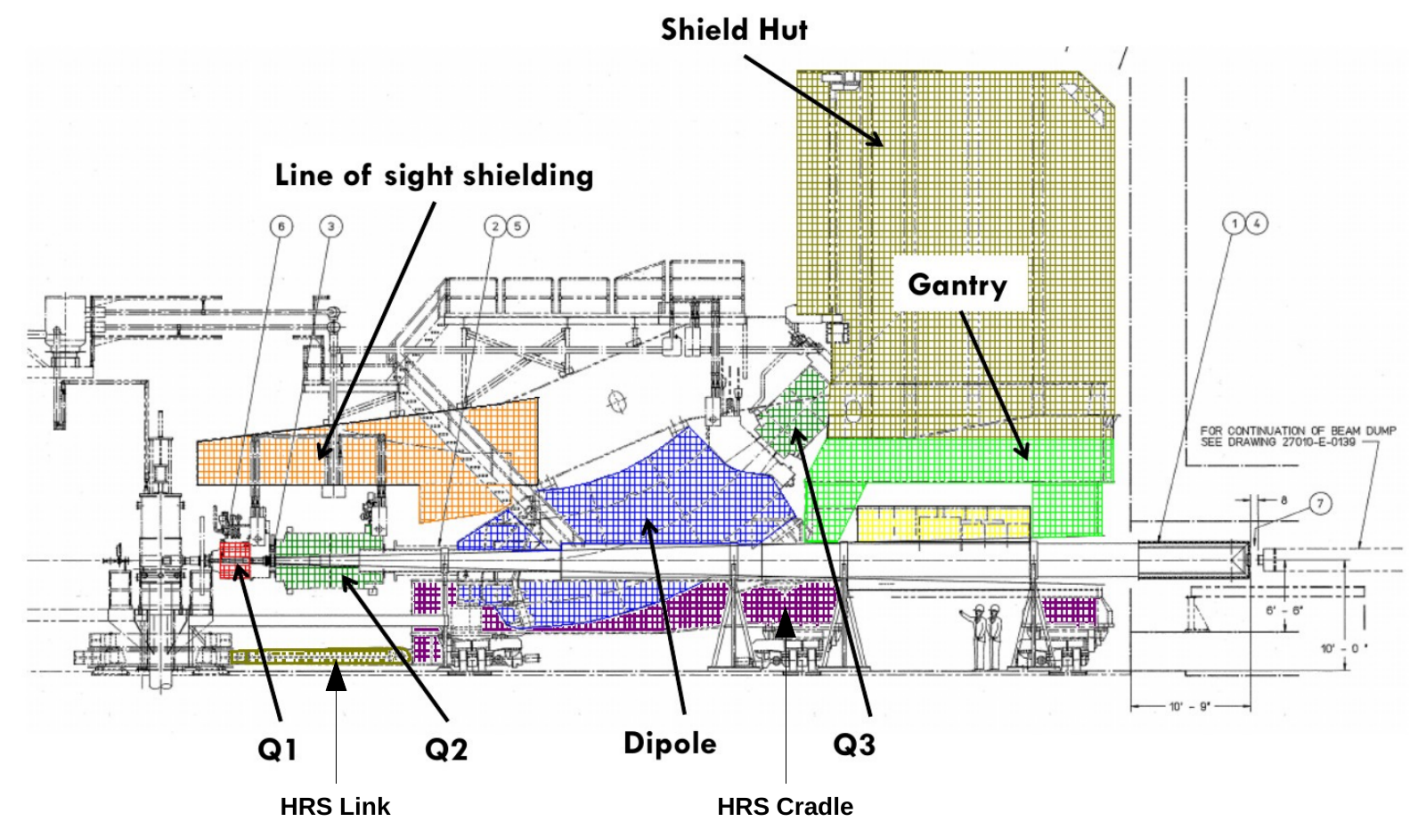

Figure 3.5: A side view of a high resolution spectrometer in JLab hall A. The components that were included in the Geant4 model are labeled.

\subsubsection{APEX experimental setup and beamline components}

As described in previous chapters, APEX measures the invariant mass spectrum of $e^{+} e^{-}$pairs produced by an incident beam of electrons on a Tungsten target. The electron will be detected in the left HRS and the positron will be detected in the right HRS. Figure 3.8 illustrates a top view of the Geant4 model of the APEX experiment. The APEX target consists of 10 ribbons of tungsten (W) or carbon $(\mathrm{C})$, and is described in detail in section 2.1. APEX uses the standard Hall A target chamber onto which is attached the APEX extension box, which is shown in Figure 3.8, and 


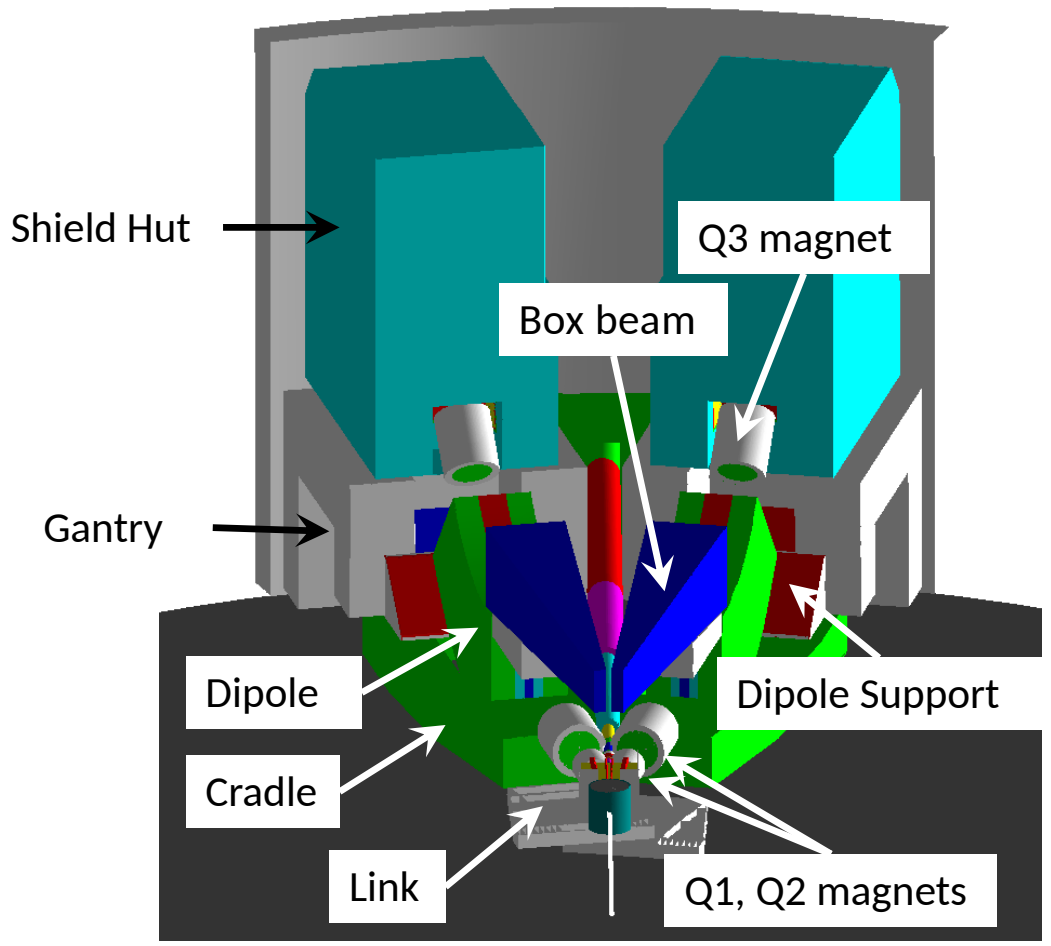

Figure 3.6: A front view of the Geant4 model of Hall A as seen from the target.

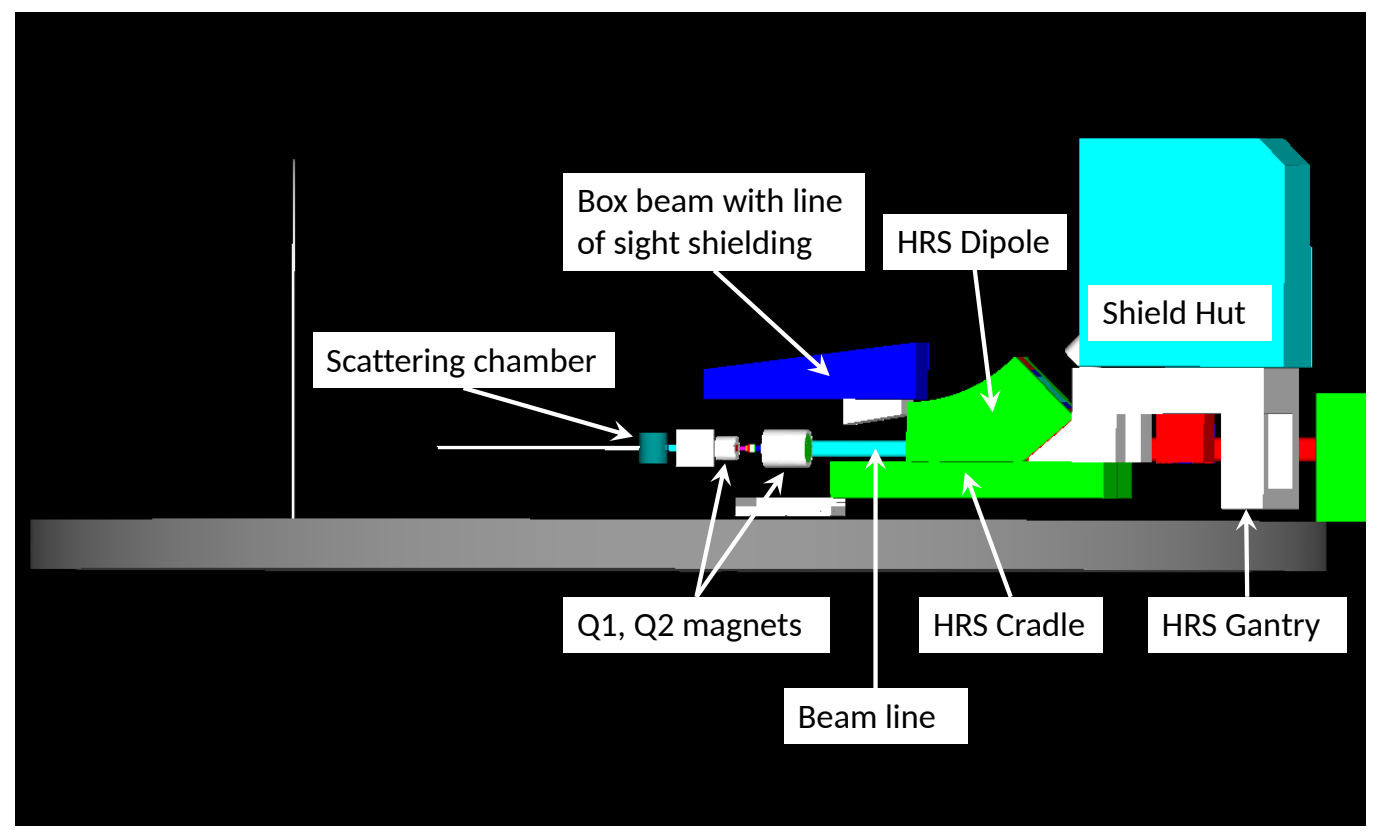

Figure 3.7: A side view of the Geant4 model of a Hall A HRS spectrometer. 
in great detail in Figure 3.9. As shown in Figure 3.9, there are three openings on the downstream face of the extension box. A beam pipe that goes through the opening at the center carries the primary electron beam. Two other openings on the downstream face of the extension box, each with a square cross section, allows the $e^{+} e^{-}$pairs to leave the extension box. A septum magnet is used in APEX for the purpose of deflecting $e^{+} e^{-}$pairs, that are produced at small angles with respect to the beam axis, into larger angles that can be detected by the HRS spectrometers.

Figure 3.10 shows the septum magnet which will be used for the APEX experiment. Figure 3.12 illustrates the Geant4 model of the new APEX septum magnet. This simplified model includes the iron core and the copper coils. The magnetic field of the septum magnet was included using TOSCA field maps. There are four field maps for four different beam energies used in the APEX experiment. Figure 3.13 shows the TOSCA field map for the APEX $4.4 \mathrm{GeV}$ setting, on top of a scaled drawing of the septum magnet. It illustrates the change in y component of the magnetic field as you go along the $\mathrm{x}$ direction. The coordinate system is given at the top right

corner of the figure and the beam goes in the positive $\mathrm{z}$ direction. Notice that the field is zero at the center of the beam pipe and outside of the magnet, so there will be no deflection in those regions.

\subsubsection{PREX-I experimental setup and beamline components}

The PREX experiment proposes to measure the neutron radius to $1 \%$, by measuring the parity violating electroweak asymmetry in the elastic scattering of polarized electrons off a ${ }^{208} \mathrm{~Pb}$ target. The first " ${ }^{208} \mathrm{~Pb}$ Radius Experiment" (PREX-I) was performed in JLab Hall A from March-July 2010 and provided the first electroweak observation of a "neutron skin" a phenomenon which is expected in a heavy, neutron- 


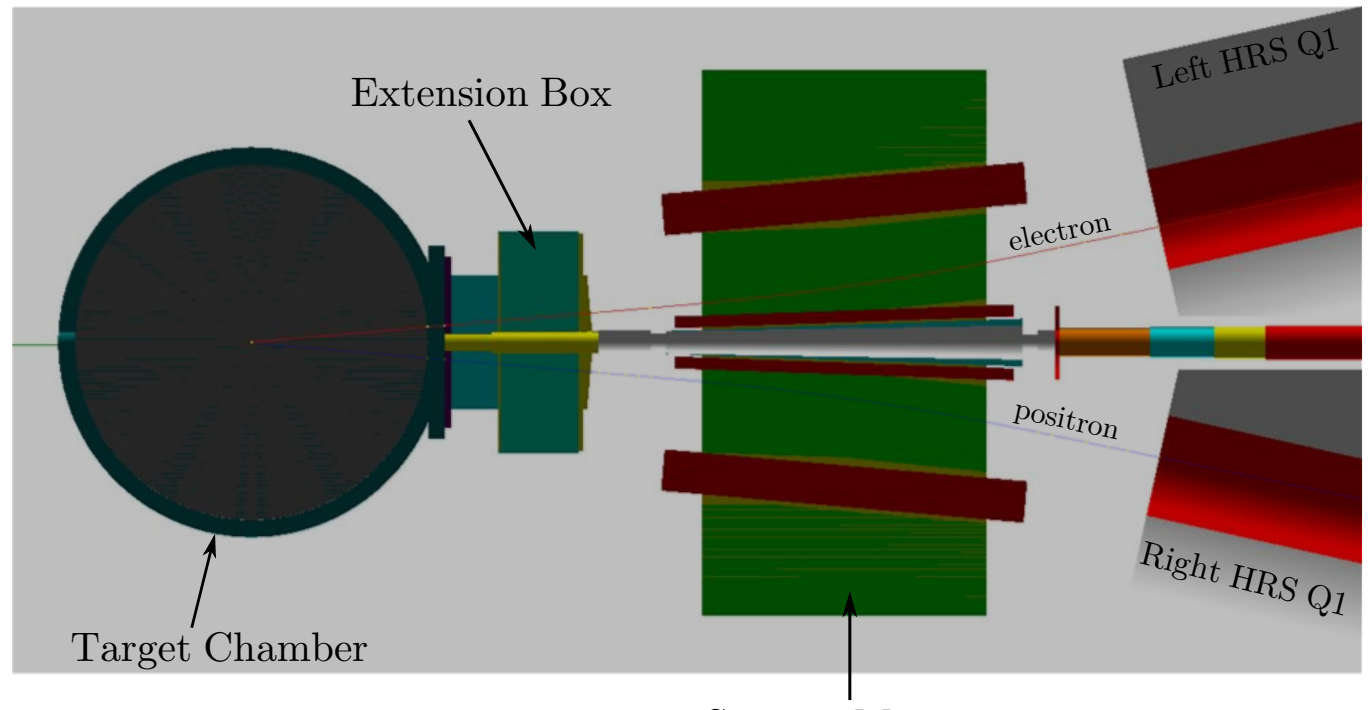

Septum Magnet

Figure 3.8: A top view of the Geant 4 model of APEX setup. Electrons and positrons leaving the target at small angles are bent by the septum magnet towards the High Resolution Spectrometers placed at $12.5^{\circ}$ with respect to the beamline.
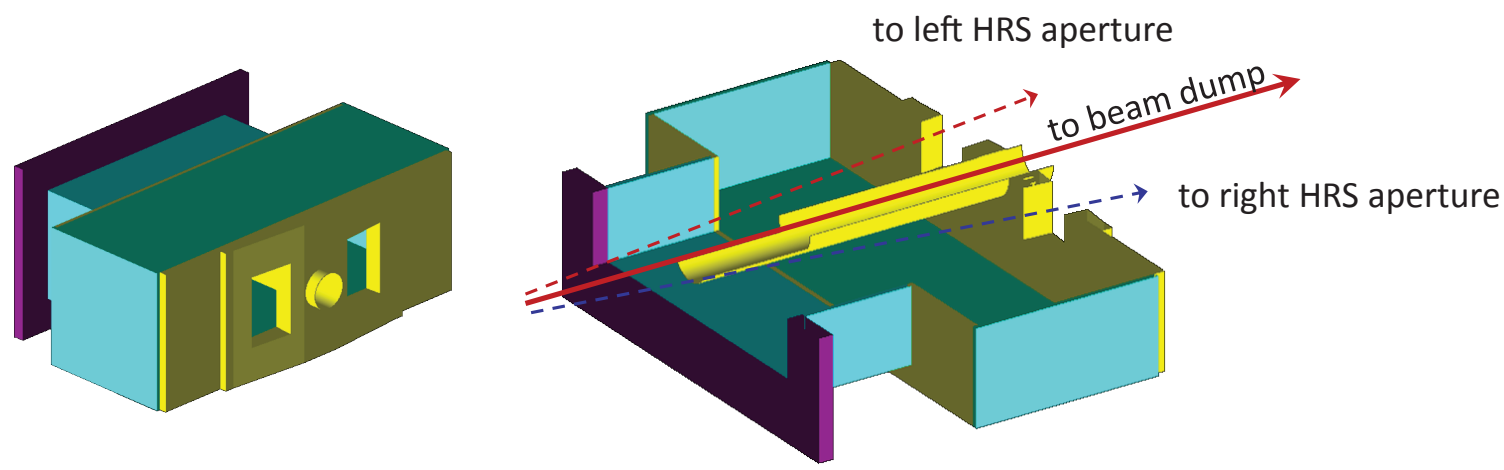

Figure 3.9: Geant4 model of APEX extension box. 

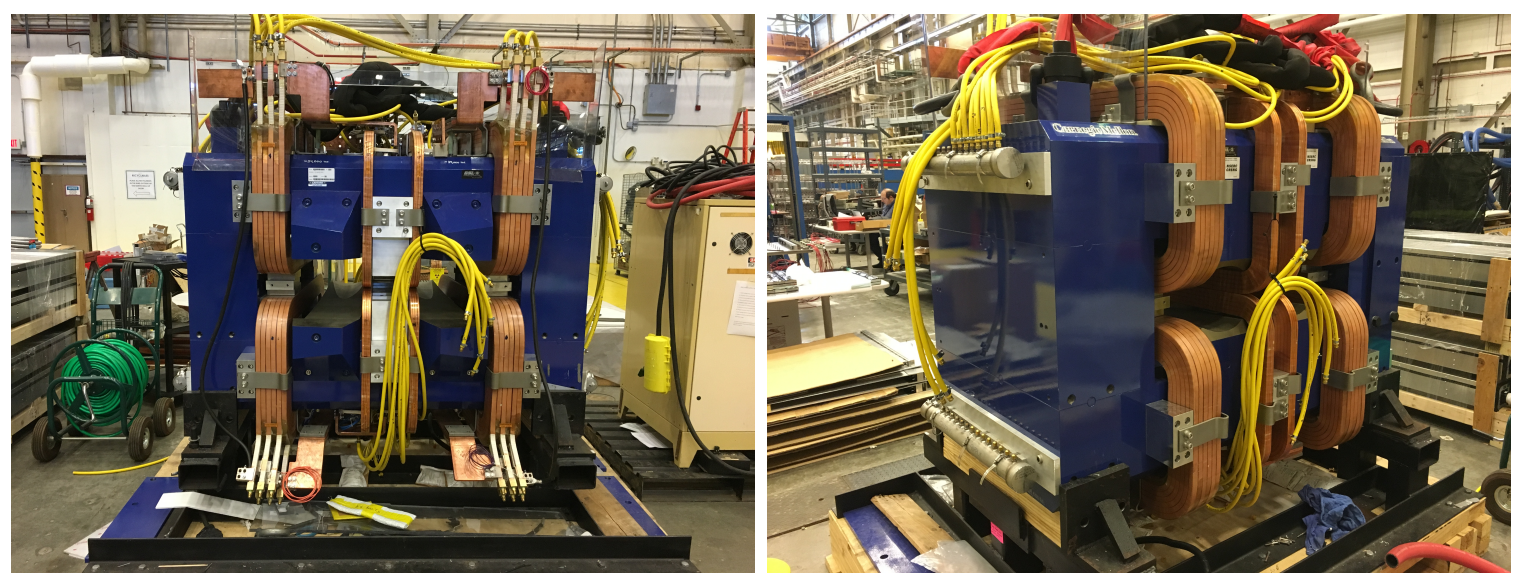

Figure 3.10: The septum magnet which will be used in the APEX experiment is shown here. Currently it is at JLab undergoing testing.
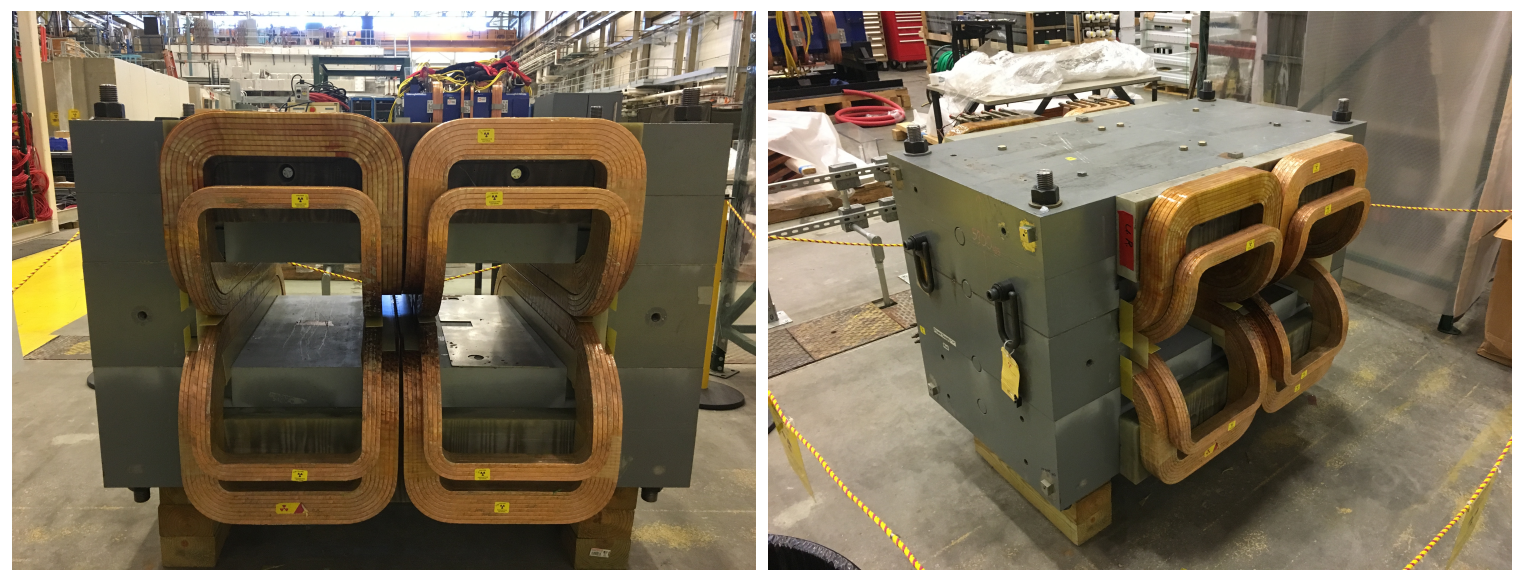

Figure 3.11: This illustrates the PREX-I septum magnet. 


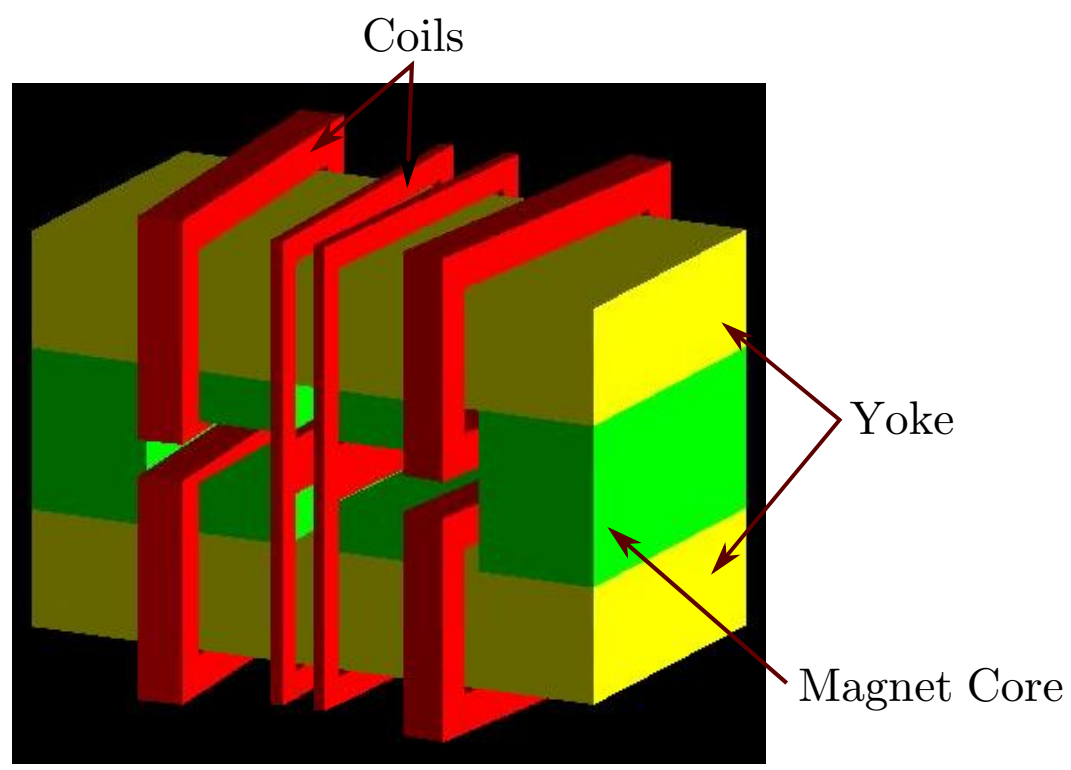

Figure 3.12: The Geant 4 model of the APEX septum magnet that was constructed for the APEX experiment. Most of the parts in the magnet is iron and the coils are made of copper. TOSCA field maps were used to apply magnetic field in to the septum magnet.

rich nucleus [41].

During PREX-I, the radiation inside the experimental hall caused significant failure of the controls systems and loss of running efficiency [40]. Specifically it caused some malfunctioning in the HRS control electronics. We simulated the PREX-I experiment and evaluated the radiation at the location of the HRS control electronics. We can consider this as a benchmark indicating excessive radiation at the HRS control electronics. That is, If the radiation produced during the APEX experiment is smaller, we can consider that the probability of damage will decrease proportionately.

In PREX-I, an electron beam of $1.06 \mathrm{GeV}$ energy was incident on a $0.5 \mathrm{~mm}(10 \%$ radiation length) thick lead disk. Both left and write HRS's measured the scattered electrons from the target and the HRS's were positioned at $12.5^{\circ}$ from the beam direction, similar to the APEX experiment. PREX-I also measured particles leaving the target at small angles. PREX-I used a different septum magnet as shown in Figure 


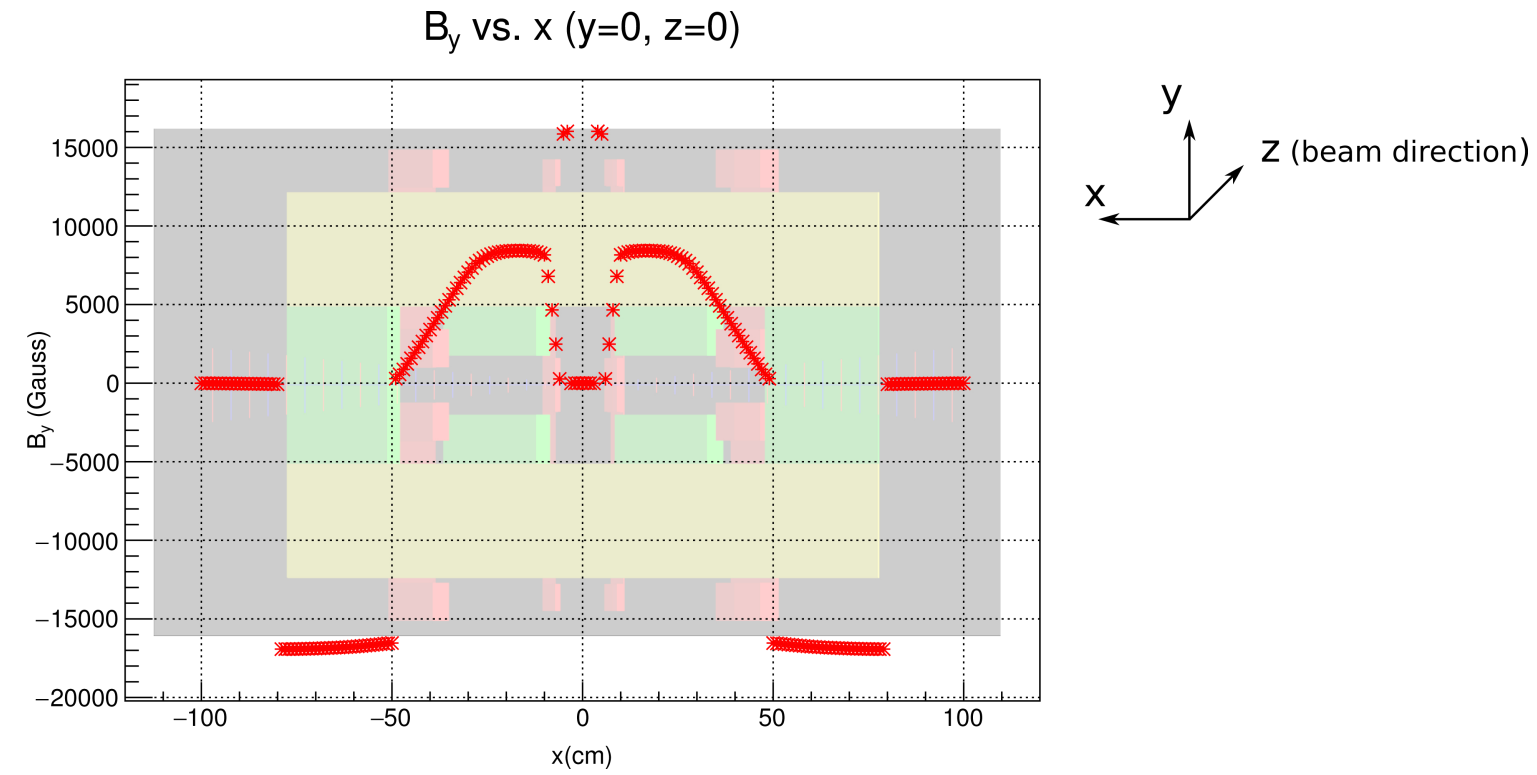

Figure 3.13: This illustrates TOSCA field map for APEX $4.4 \mathrm{GeV}$ setting. The y component of the magnetic field along the $\mathrm{x}$-axis is shown in the plot. The image of the septum magnet in the background and the coordinate system used in the simulation are provided as a guide. 
3.11. In our simulations we used the APEX septum magnet instead as they are similar in geometry. For the magnetic field we used the TOSCA field map generated for the 1.1 GeV setting of APEX, but with the opposite polarity for the field values on the left side of the septum (since PREX only deflects electrons). Figure 3.14 shows the modified field map used in the PREX-I simulation. The PREX-I experiment used a Tungsten collimator placed inside the PREX-I "extension box". Figure 3.15 and Figure 3.16 illustrate the Geant4 model of the PREX-I extension box and the PREXI collimator respectively. Figure 3.17 shows the final PREX-I setup used for the simulation. Implications of the PREX-I geometry will be discussed later when we discuss results.

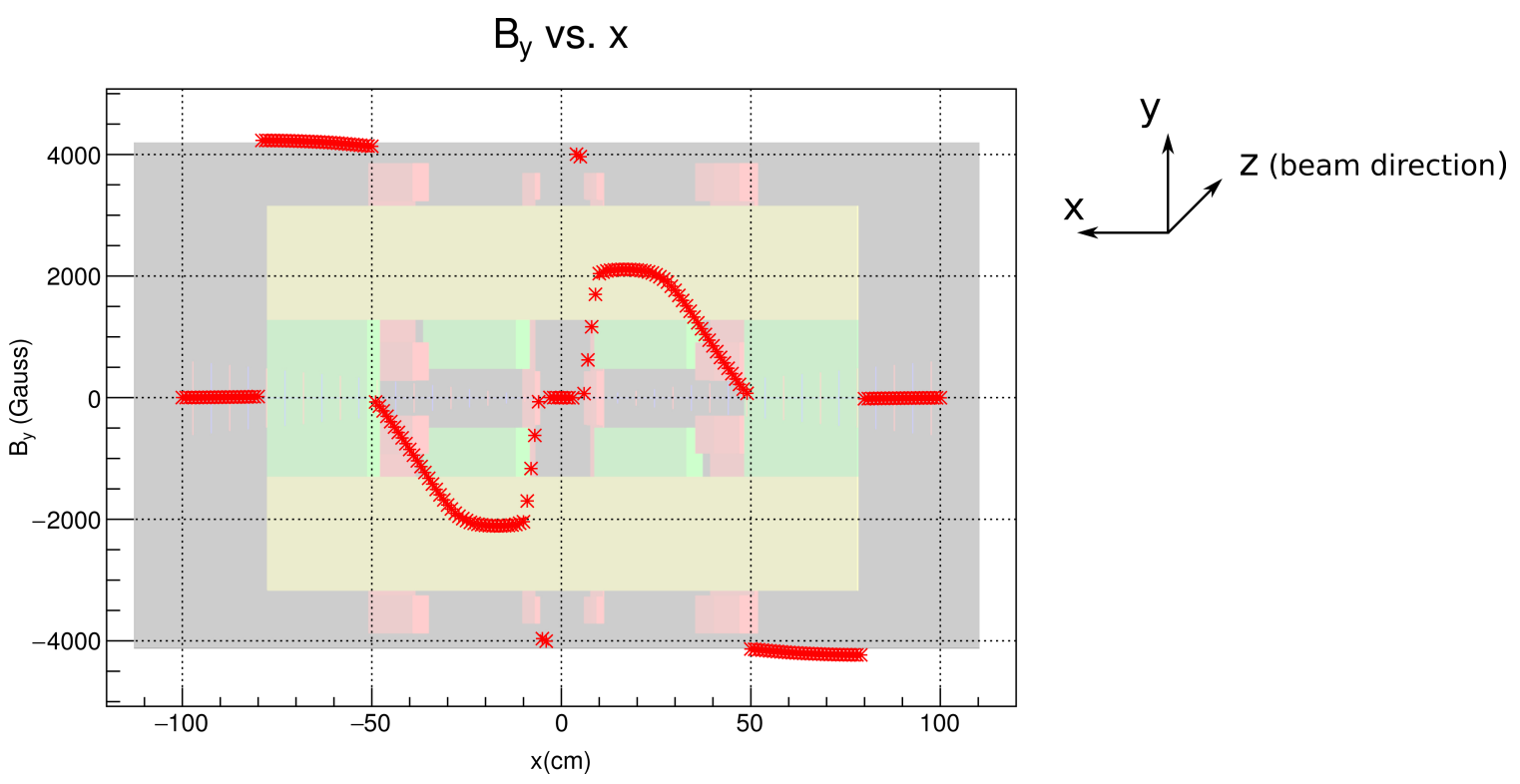

Figure 3.14: This illustrates TOSCA field map used for the PREX-I experiment. The y component of the magnetic field along the $\mathrm{x}$-axis is shown in the plot. The image of the septum magnet in the background and the coordinate system used in the simulation are provided as a guide. 

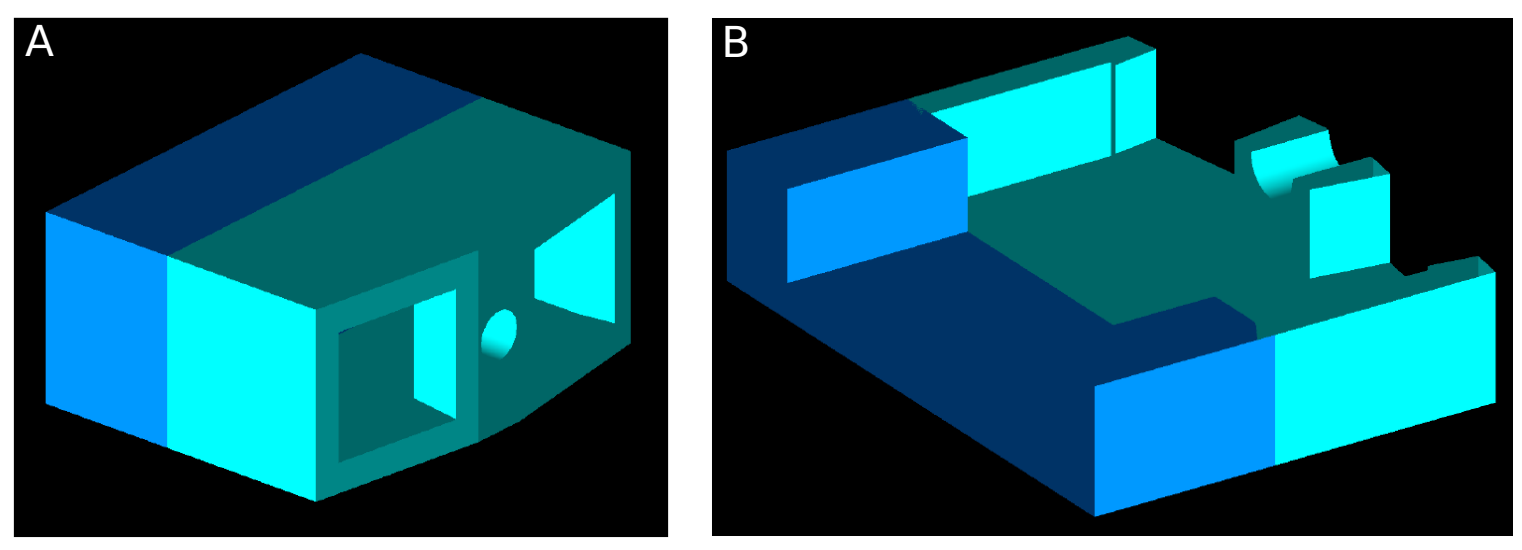

Figure 3.15: The Geant4 model of the PREX extension box. The Tungsten collimator was placed inside this extension box.

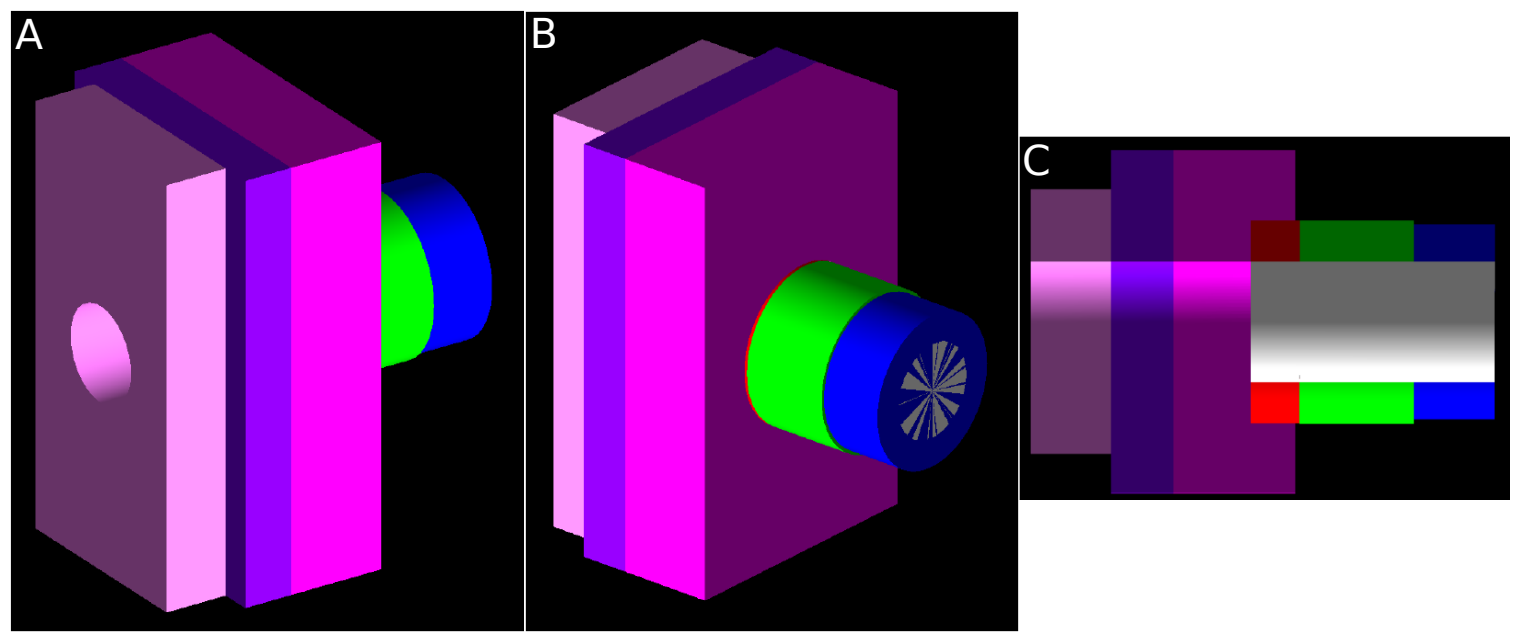

Figure 3.16: The Geant4 model of the PREX collimator. 


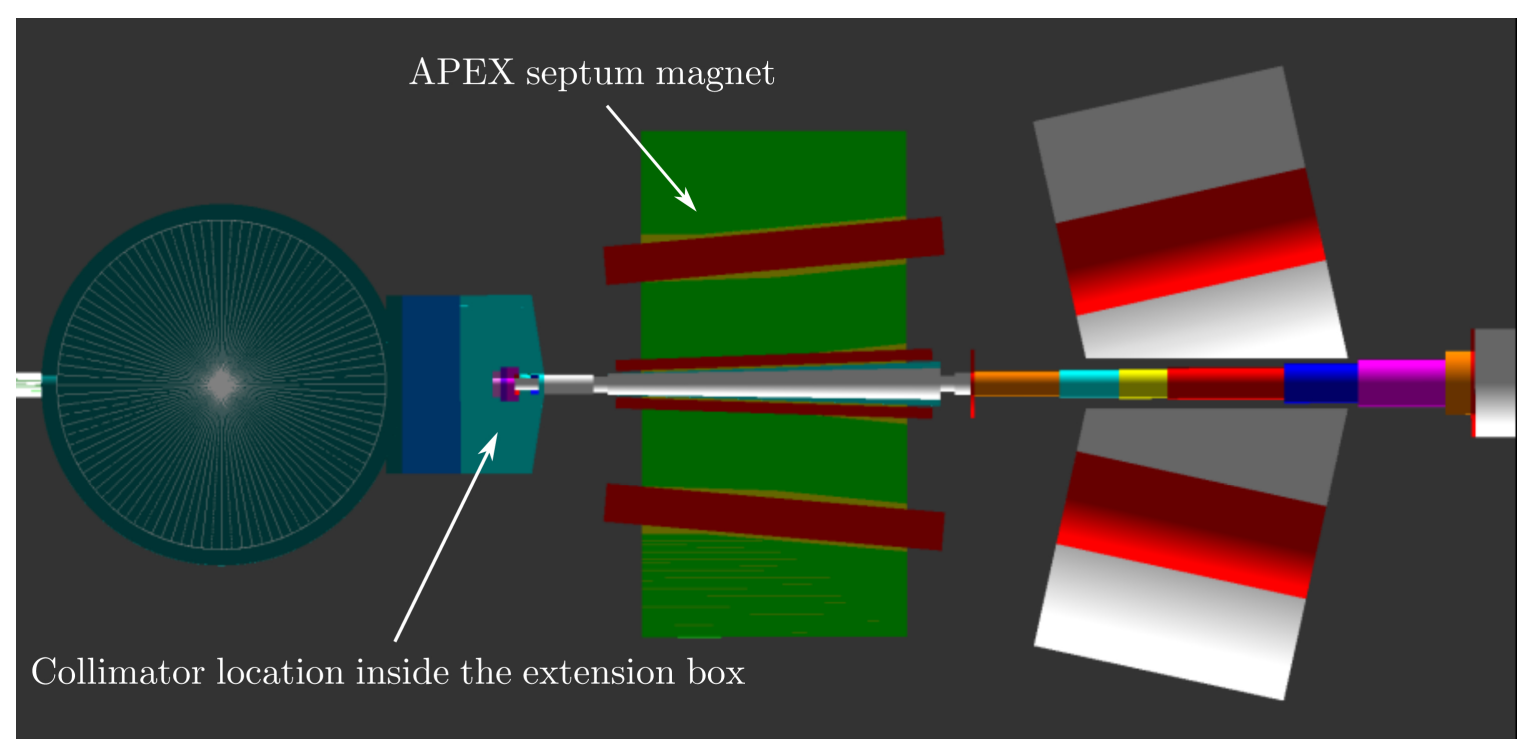

Figure 3.17: This illustrates the PREX-I experiment setup. The tungsten collimator sits inside the PREX-I extension box. Some details of the HRS spectrometer are removed for clarity of the image.

\subsubsection{Real Compton Scattering (RCS) experimental setup and beamline components}

Jefferson Lab experiment E99-114, also known as the Real Compton Scattering (RCS) experiment, which ran in the year 2002, explored the scattering of few- $\mathrm{GeV}$ photons off the proton at large scattering angles [42]. In this experiment, a longitudinally polarized electron beam with an energy of $3.48 \mathrm{GeV}$ and a current of $40 \mu \mathrm{A}$ was incident on a $0.81 \mathrm{~mm}$ thick copper radiator. The mixed beam of electrons and bremsstrahlung photons were incident on a $15 \mathrm{~cm}$ long liquid $\mathrm{H}_{2}$ target, located just downstream of the radiator. The scattered photon was detected at a mean scattering angle of $65^{\circ}$ in a calorimeter and the associated recoil proton was detected in one of the Hall A high resolution spectrometers.

The RCS experiment was chosen for use as a $2^{\text {nd }}$ benchmark for the APEX radia- 
tion studies. The RCS experiment had a smooth run without any issues of radiation damage. If the level of radiation during APEX is lower than the radiation during the RCS, it would be ideal. Later we realized an experiment like HAPPEX would be a better candidate for this benchmark.

For the RCS simulation, no additional beamline components were needed. Figure 3.18 illustrates the RCS setup used for the simulation. To simplify the model we did not include the calorimeter, which we do not believe significantly affected our results.

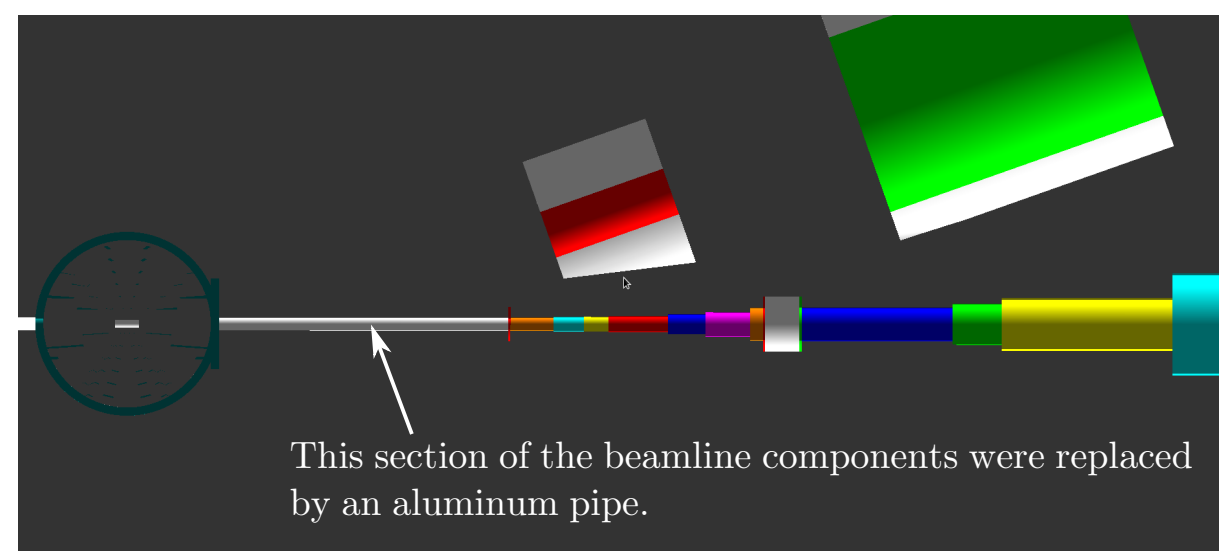

Figure 3.18: Geant4 model of the RCS experiment. The model was simplified by not including the deflection magnet and the calorimeter. Neither an extension box nor a septum magnet was used in the experiment.

\subsection{Relative comparisons of APEX with other ex- periments}

In this section, we will discuss our results of the Geant4 simulation developed for the APEX radiation study. The method of calculating "particle fluence" and the concept of "equivalent $1 \mathrm{MeV}$ neutron fluence" will be discussed. The calculated 
APEX radiation levels at key locations in JLab Hall A were compared with similar calculations for both PREX-I and RCS to get a relative comparison of results. The calculated APEX radiation level at the HRS control electronics was a factor 9 smaller than the PREX-I, resulting a lower damage to semiconductor electronics.

\subsubsection{Particle fluence spectra}

Particle fluences were calculated at different locations in JLab Hall A using our Geant4 simulations. Several scoring-volumes were positioned in the Geant4 Hall A model. As introduced in section 3.1, a scoring-volume is a volume in any shape or size that has the capability of recording information about the particles that pass through it. Scoring-volumes that were used in the APEX simulation recorded particle information such as kinetic energy, momentum direction, particle ID and track length. Particle fluence at a particular location in the JLab Hall A model was calculated using the volume-method we discussed in section 3.1.

Two important locations at which we calculated particle fluence were $\sim 16 \mathrm{~m}$ upstream and $\sim 20 \mathrm{~m}$ downstream from the Hall A center. To evaluate the upstream particle fluence, we used a semi-circular shaped scoring-volume with a radius of $15 \mathrm{~m}$ and a thickness of $5 \mathrm{~cm}$, positioned at $16 \mathrm{~m}$ upstream from the Hall A center. Figure 3.19(A) shows the positioning of the semi-circular shaped scoring-volume in the Geant4 model. The downstream particle fluences were calculated using another scoring-volume in the shape of a cube with a size of $2 \mathrm{~m} \times 2 \mathrm{~m} \times 2 \mathrm{~m}$. This was positioned near where the HRS control electronics are located as shown in Figure $3.19(\mathrm{~B})$. 


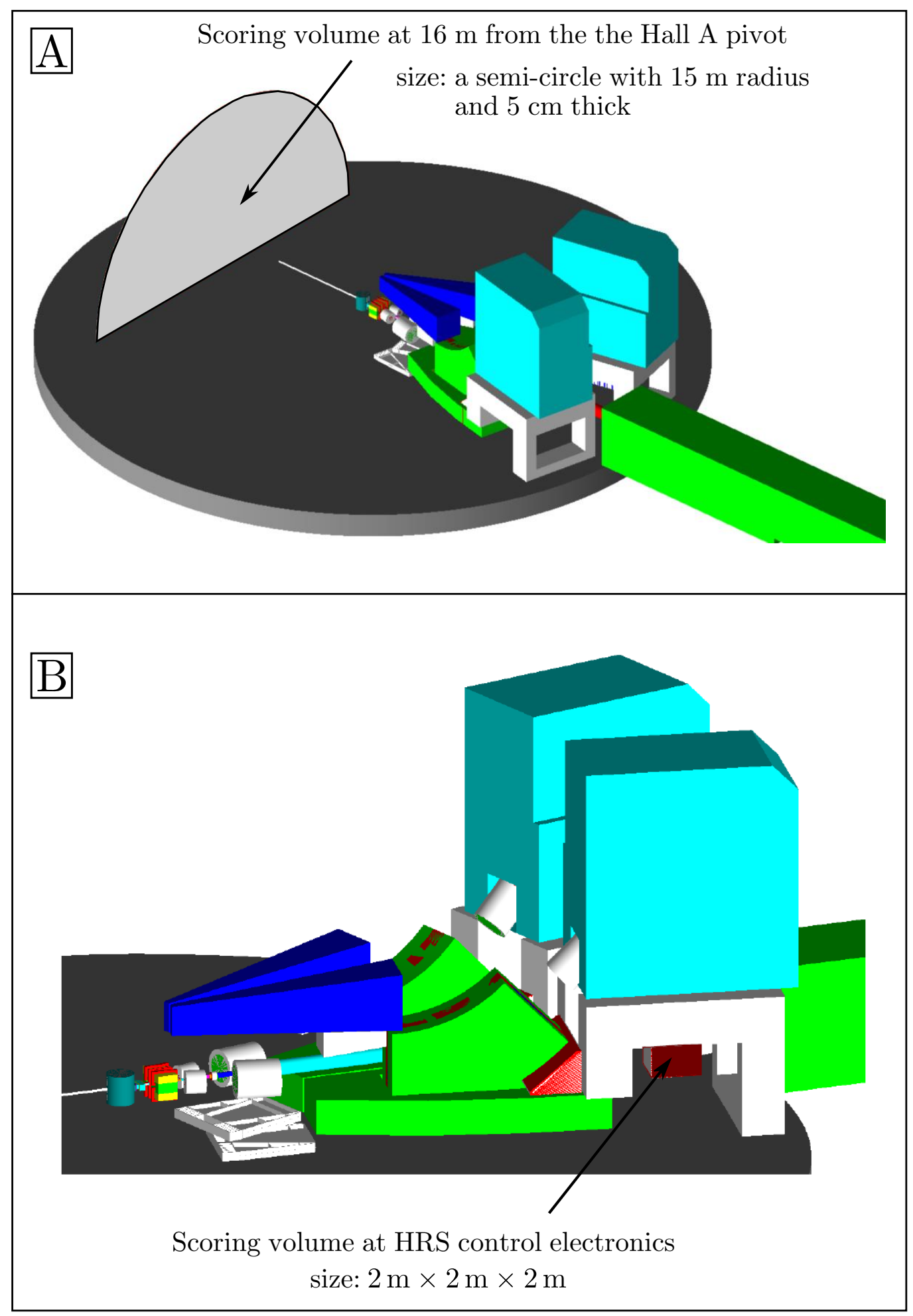

Figure 3.19: This figure shows the scoring-volumes used in the Geant4 model. A semi-circular shaped scoring-volume was used in the simulation to detect particles in the upstream region. We used a large volume to increase statistics. A $2 \mathrm{~m} \times 2 \mathrm{~m} \times 2 \mathrm{~m}$ box was used as the scoring-volume in downstream region. 


\subsubsection{Radiation damage to semiconductor electronics}

Extremely harsh radiation environments in high-energy and medium-energy particle accelerators poses a challenge to the use of semiconductor electronics, especially during high -luminosity experiments such as APEX. Radiation can cause an increase of both the reverse current and the necessary depletion voltage as well as a decrease of the charge collection efficiency in semiconductors [53]. These effects can either completely destroy the equipment or cause temporary malfunction, that results in losing both time and money.

When an energetic particle is incident on a semiconductor, it loses energy via ionization and non-ionization processes. It produces electron-hole pairs if the process is ionization. Non-ionization processes will displace atoms from their primary lattice positions to interstitial sites, creating vacancies. Interstitial is the defect caused by an atom moving to a non-lattice position [52]. A vacancy and an adjacent interstitial is called a Frenkel pair. The damage due to the generation of frenkel pairs, also known as the bulk damage, is the main type of radiation damage to accelerator electronics.

The radiation-induced defects which are relatively far apart are referred to as point defects. Neutrons and electrons with energies $\sim 175 \mathrm{eV}$ and $\sim 260 \mathrm{keV}$ respectively

are sufficient to cause this phenomenon [53]. If a significant amount of energy is transferred to the initial atom, then this dislodged atom can displace other atoms nearby creating a region of defects. These are referred to as defect clusters. Neutrons with energy above $\sim 35 \mathrm{keV}$ and electrons above $\sim 8 \mathrm{MeV}$ can cause defect clusters. These defects will give rise to new energy levels and those energy levels alter material electrical and optical properties. Some of these types of damage are reversible and some become a permanent.

Radiation response can be predicted reasonably well based on analytical calcula- 
tions of the amount of displacement-damage energy imparted to the primary atom. Based on these analytical calculations, if we could correlate the damage induced by one particle type, with damage induced by another, that could save time on radiation testing. There are many studies done to understand the bulk damage to Silicon from hadrons and high energy leptons [57] [54]. They have led to the assumption that silicon damage due to different types of radiation is proportional to a quantity known as Non Ionization Energy Loss or NIEL. NIEL is a function of energy, the type of incident radiation and the material in question. The product of NIEL and target thickness yield energy loss. The quantity NIEL can be calculated analytically from first principles based on differential cross sections and interaction kinematics. NIEL can be calculated using the following analytical expression which sums elastic and inelastic contributions [52],

$$
\mathrm{NIEL}=\left(\frac{N}{A}\right)\left[\sigma_{e} T_{e}+\sigma_{i} T_{i}\right]
$$

where $\sigma_{e}$ and $\sigma_{i}$ are total elastic and inelastic cross sections of atomic displacement. $T_{e}$ and $T_{i}$ are elastic and inelastic effective average recoil energies corrected for ionization loss, respectively. $N$ is Avogadros number, and $A$ is the gram atomic weight of the target material. NIEL is usually presented in units of $\mathrm{keVcm}^{2} / \mathrm{g}$. With this choice of units, target thickness is expressed in units of $\mathrm{g} / \mathrm{cm}^{2}$. NIEL can also be specified in so called displacement damage cross section (D) which is expressed in MeVmb. For silicon the relation between $\mathrm{D}$ and NIEL is $100 \mathrm{MeVmb}=2.144 \mathrm{keVcm}^{2} / \mathrm{g}$.

There are many published analytical calculations for NIEL for different materials and particle types. Examples include, Dale et al. [55], Huhtinen et al. [56], Summers et al. [57], Akkerman et al. [58] and Van Ginneken et al. [59]. A. Vasilescu and G. Lindstroem have put together a very useful online compilation of displacement 
damage in silicon from selected sources [60]. It is standard to express Silicon damage in terms of the fluence of $1 \mathrm{MeV}$ neutrons, $\Phi_{\mathrm{eq}}^{1 \mathrm{MeV}}$, which would cause that damage (fluence has units of $\# / \mathrm{cm}^{2}$ ) [61][62]. This can be given by the equation,

$$
\Phi_{\mathrm{eq}}^{1 \mathrm{MeV}}=\int_{0}^{\infty} \frac{\mathrm{D}(\mathrm{E})}{\mathrm{D}(1 \mathrm{MeV})} \phi(\mathrm{E}) \mathrm{dE}
$$

where $\mathrm{D}(\mathrm{E}) / \mathrm{D}(1 \mathrm{MeV})$ is the normalized displacement damage cross-section. Here $\mathrm{D}(1 \mathrm{MeV})=95 \mathrm{MeV} \mathrm{mb}$. Figure 3.20 shows normalized silicon displacement damage functions for neutrons, protons, electrons and pions [60]. As shown in equation 3.2, the convolution of an arbitrary particle fluence with the displacement damage curve for silicon gives us the fluence of $1 \mathrm{MeV}$ neutrons which does the same amount of damage as the arbitrary particle fluence.

\subsubsection{Comparison of APEX radiation levels}

The main goal of this work was to study the radiation backgrounds we expect during the APEX experiment and to keep the radiation levels lower than the threshold values for semiconductor electronic damage. This study was a major part of the APEX readiness review in which APEX was approved for beam time. In Figure 3.21 , threshold levels for radiation damage in several materials including Silicon are shown [65]. These threshold levels are given in fluences of $1 \mathrm{MeV}$ neutrons. JLab has established an acceptable radiation level for equipment of $10^{13} \mathrm{n}_{\mathrm{eq}} \mathrm{cm}^{-2}$, consistent with Figure 3.21, which is delineated in ESAD [66]. However, a recent study show that the soft electronics such as optocouplers could be damaged even at lower radiation level of $10^{11} \mathrm{n}_{\mathrm{eq}} \mathrm{cm}^{-2}$ [69].

Figure 3.22 illustrates a top view of JLab hall A. In this illustration, the electron beam goes from left to right at $\sim 3 \mathrm{~m}$ above the Hall A floor. The two HRS's are 


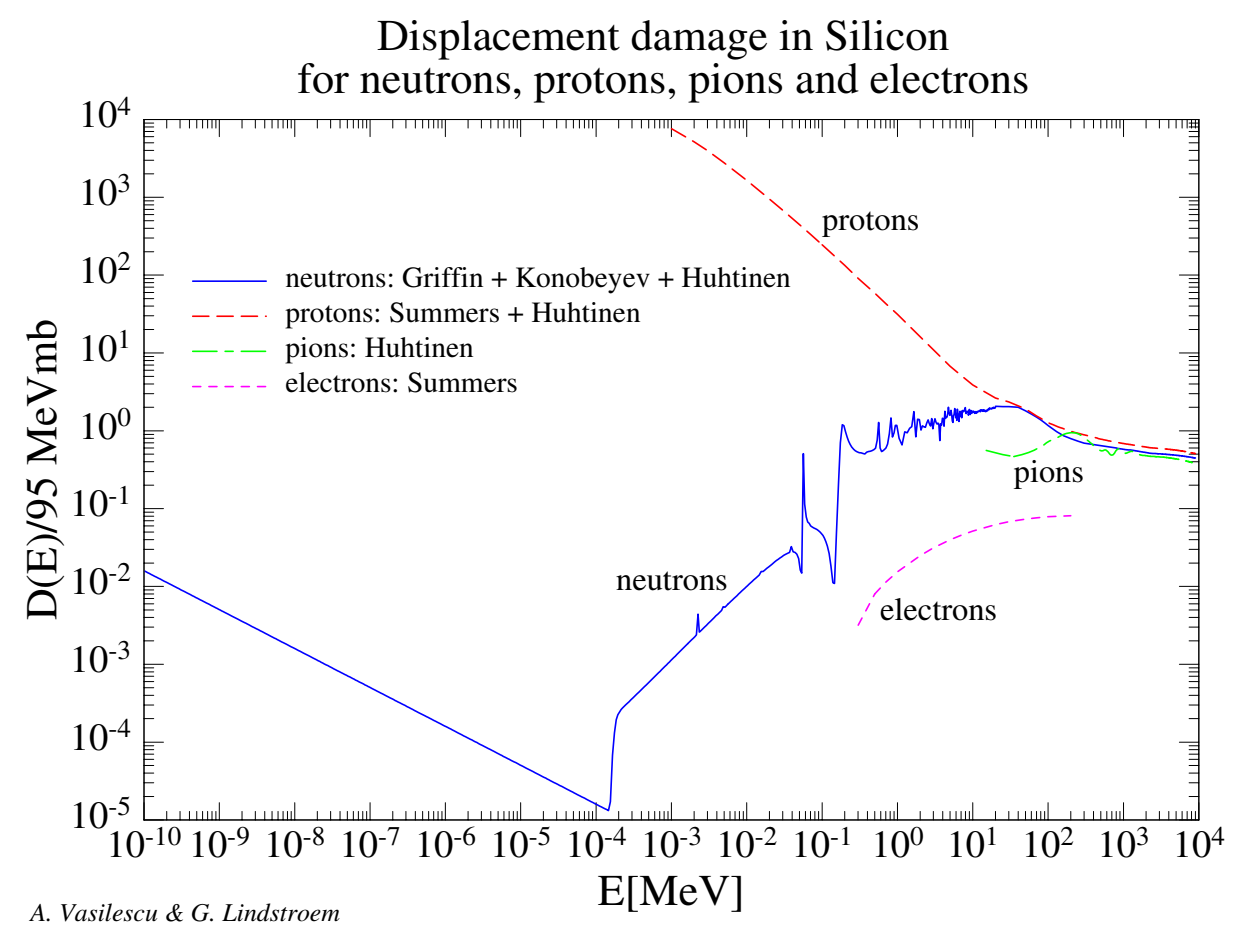

Figure 3.20: Silicon displacement damage functions for neutrons, protons, electrons and pions. Values are normalized to the displacement damage by $1 \mathrm{MeV}$ neutrons (95 MeVmb). 


\section{Radiation Damage to Materials/Electronics}

!!! A Rough Overview Only !!!

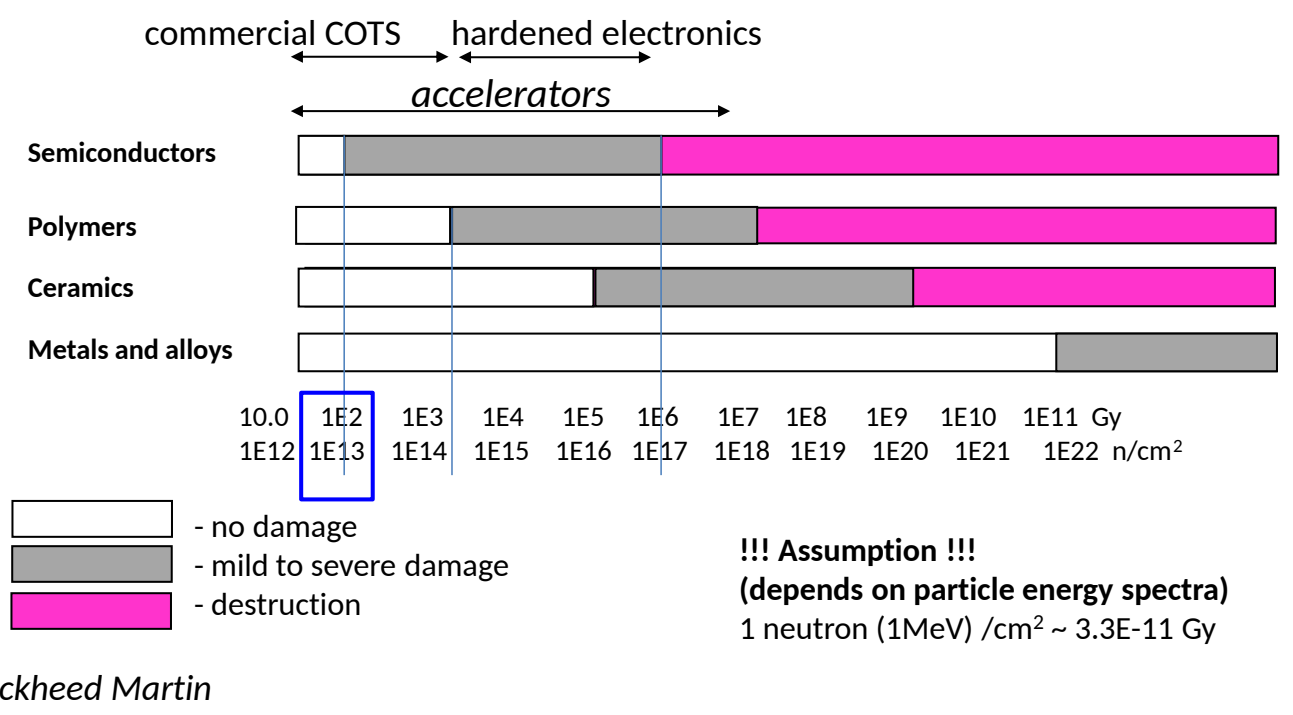

Figure 3.21: A rough estimate for $1 \mathrm{MeV}$ neutron fluence thresholds for different material/electronics.

on either side of the beam line, and can be rotated around the Hall A center, to detect $e^{+} / e^{-}$pairs at different angles. These $e^{+} / e^{-}$pairs will be bent vertically and will be detected in the detectors located in the shield houses at a height of $\sim 10 \mathrm{~m}$ above the Hall A floor. The electronics that are associated with each HRS are located $\sim 7 \mathrm{~m}$ below the shield house. These two locations are labeled in Figure 3.22 as "HRS control and power electronics". This is the location where most of the semiconductor electronics are housed and from past experience we know that this area has the highest radiation risk in the hall. Figure 3.23 shows a photograph of one of the HRS's and the location of the HRS control electronics is indicated.

As described in section 3.3.1, we used a scoring volume of a $2 \mathrm{~m} \times 2 \mathrm{~m} \times 2 \mathrm{~m}$ at the location of the HRS control electronics to calculate neutron fluence. We calculated the $1 \mathrm{MeV}$ equivalent neutron fluence at the HRS control electronics using the method described in section 3.3.2 for all the proposed APEX settings. Figure 3.24 shows 


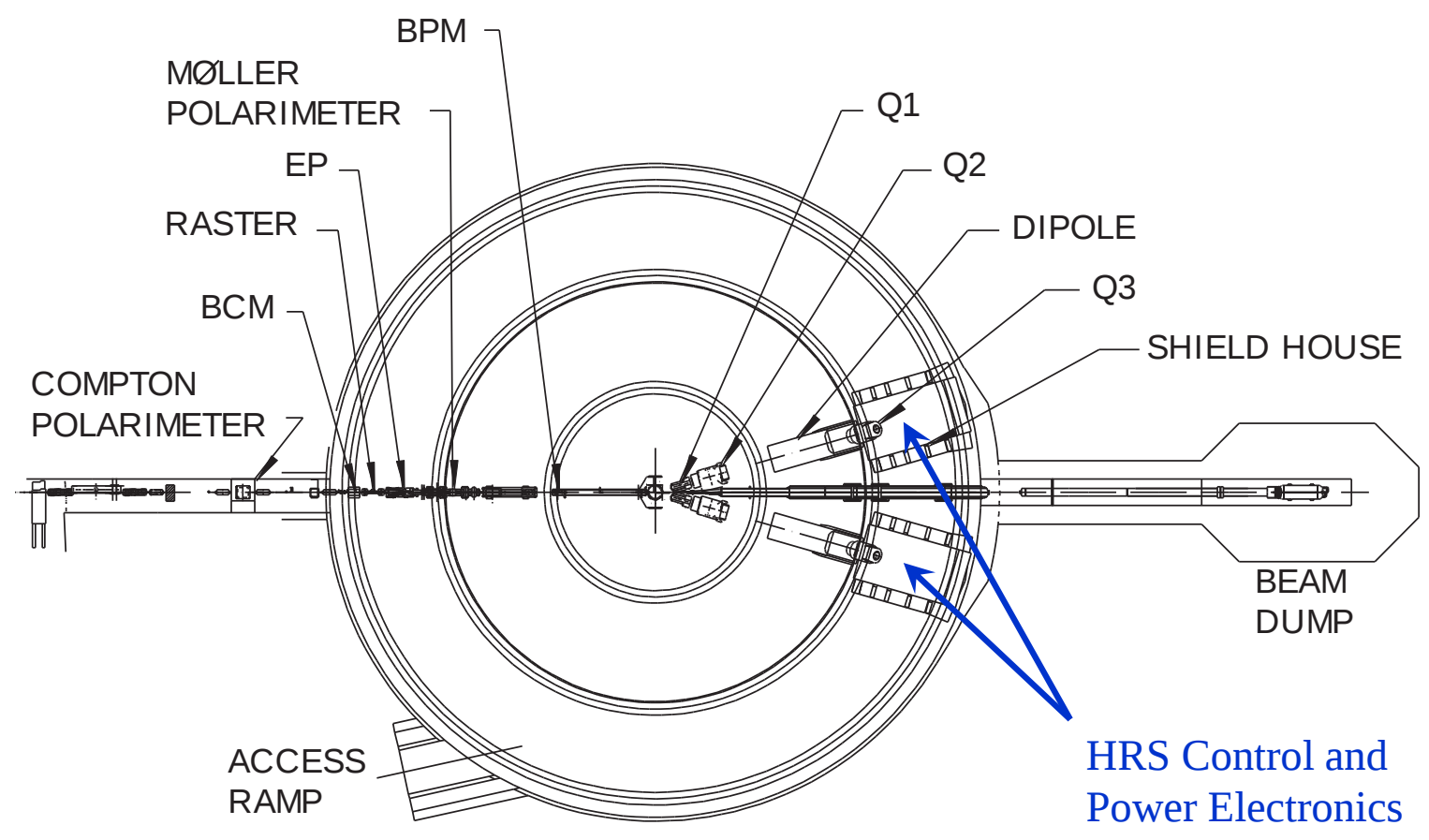

Figure 3.22: Drawing illustrates the top view of the Jefferson Lab experimental hall A equipment. Most of the radiation sensitive semiconductor electronics are located at the position labeled as "HRS control and power electronics". This is the main point where we evaluated radiation damage. 


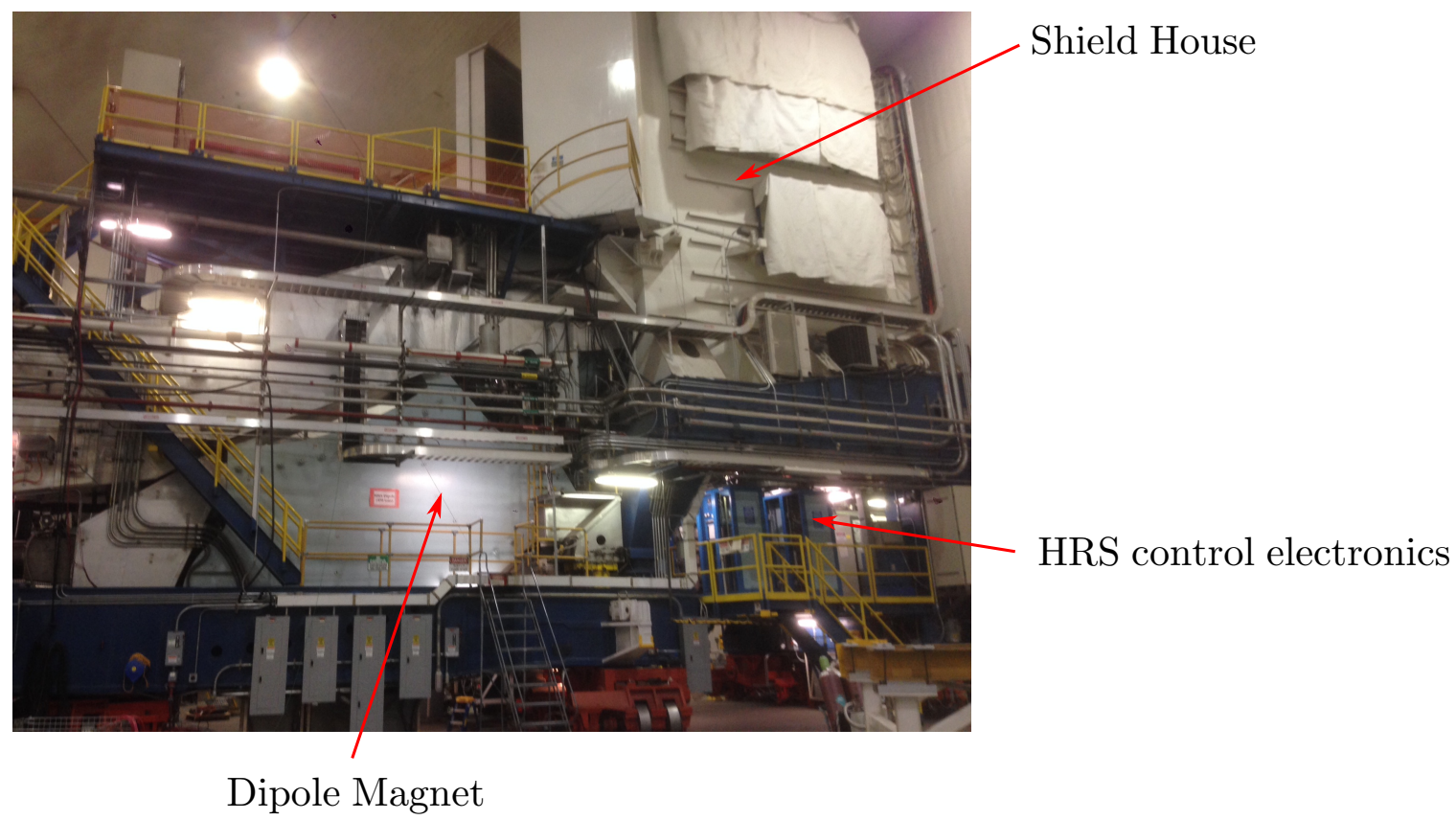

Figure 3.23: Figure shows a photograph of the HRS with a view of the HRS control electronics. Each HRS has its own control electronics at the back of the HRS just under the shield hut. This area also moves with the HRS when it rotates around the hall A pivot. 
several plots summarizing the radiation calculations for the APEX $3.3 \mathrm{GeV}$ setting which was specified to have a $5.3 \%$ r.l. Tungsten target. The dashed (black) line in the plot indicates the neutron fluence-rate spectrum at the HRS control electronics. The solid (red) line represents the original (black) spectrum weighted by the Silicon damage curve shown in Figure 3.20. Thus, the sum of all the bins in the solid (red) curve yields the equivalent fluence rate of $1 \mathrm{MeV}$ neutrons. Based on these plots, we can predict that the highest contribution to silicon damage will come from the neutrons with energies above $0.1 \mathrm{MeV}$. The dotted (blue) line indicates the cumulative silicon damage as a percentage and it is useful when calculating the fraction of damage from each part of the neutron spectrum. For instance, in the APEX $3.3 \mathrm{GeV}$ setting, the total damage dose rate at the HRS electronics is $2.0 \times 10^{4} \mathrm{n}_{\mathrm{eq}} \mathrm{cm}^{-2} \mathrm{~s}^{-1}$, and the contribution from the neutrons with energies between $1 \mathrm{MeV}$ and $10 \mathrm{MeV}$ is $30 \%$ of the total damage rate. Similarly, Figure 3.25 illustrates the $e^{+} / e^{-}$fluence rate at HRS control electronics and its damage fluence. Figure 3.26 shows photon energy spectrum at HRS electronics for the APEX $3.3 \mathrm{GeV}$ setting. The silicon damage due to photons was not calculated due to the unavailability of fluence-to-silicon damage curves. Several studies have shown that, for a given fluence, the silicon damage due to photons is lower than the neutrons [58] [57]. Based on the duration of the experiment, we can calculate the total damage dose in terms of an equivalent fluence of $1 \mathrm{MeV}$ neutrons. Table 3.3 summarizes our radiation calculations at the location of the HRS control electronics for all the simulated experiments. In the Table 3.3, it can be seen that the total silicon damage is dominated by the damage due to neutrons. The calculations in Table 3.3 also allows us to compare different experiments. Based on this, we see that the total silicon damage for all the APEX settings is about an order of magnitude lower than the PREX-I experiment.

As shown in the Table 3.3, the calculated $1 \mathrm{MeV}$ equivalent neutron fluences at 


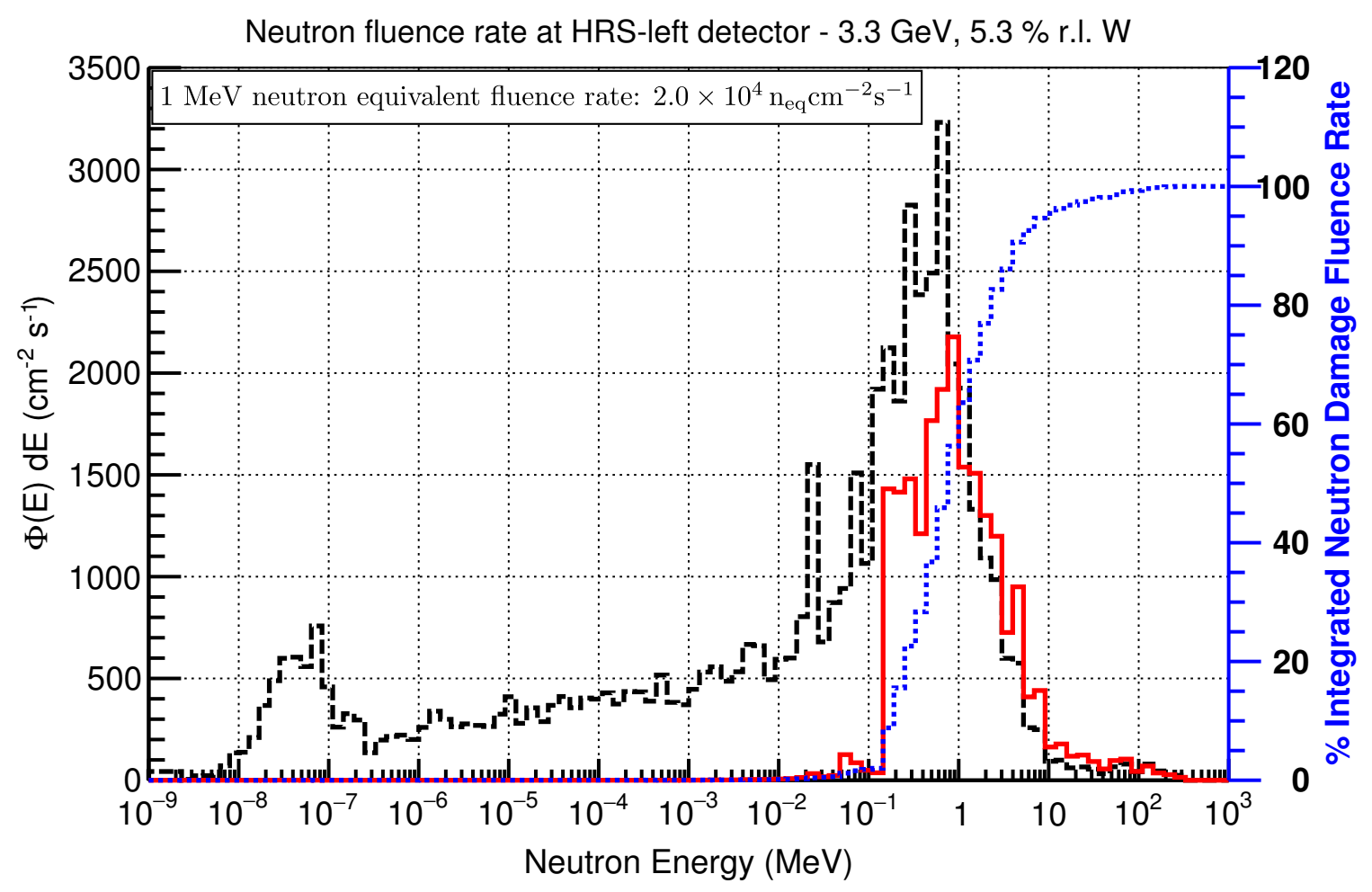

Figure 3.24: The dashed (black) line shows the simulated neutron fluence-rate spectrum at the location of the HRS control electronics for the APEX experiment with $3.3 \mathrm{GeV}$ setting. Beam current was assumed to be $120 \mu \mathrm{A}$. The histogram has equal-width bins in the log scale and the fluence is per energy bin which is indicated by $\Phi(E) d E$. The solid (red) line represents the original (black) neutron spectrum weighted by the Silicon damage curve. The dotted (blue) line shows the cumulative silicon damage as a percentage and it is indicated by the secondary axis on the right side of the plot. 


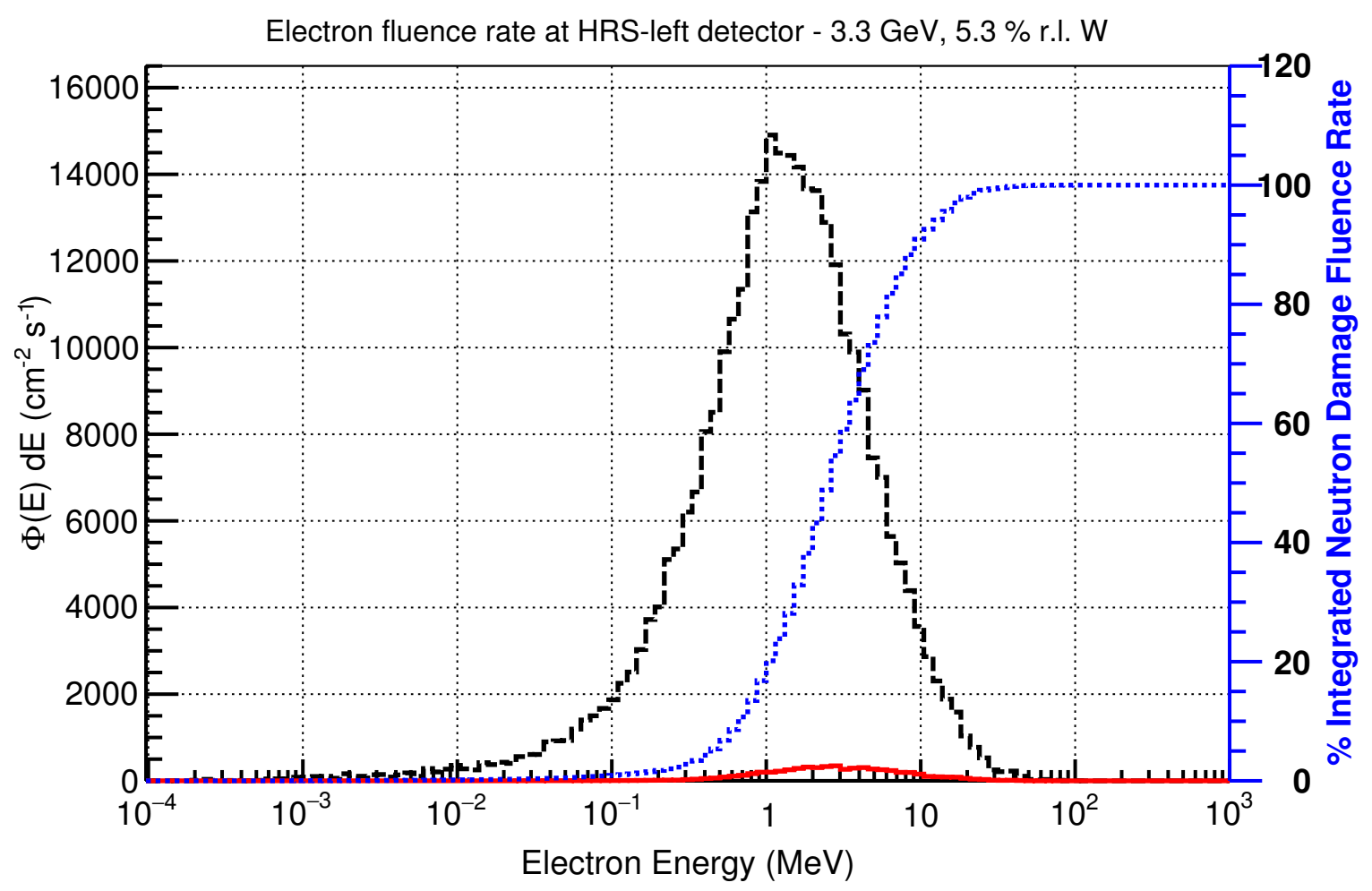

Figure 3.25: The dashed (black) line shows the simulated electron fluence-rate spectrum at the location of the HRS control electronics for the APEX experiment with the $3.3 \mathrm{GeV}$ setting. Beam current was assumed to be $120 \mu \mathrm{A}$. The histogram has equal-width bins in the log scale and the fluence is per energy bin which is indicated by $\Phi(\mathrm{E}) \mathrm{dE}$. The solid (red) line represents the original (black) electron spectrum weighted by the Silicon damage curve. The dotted (blue) line shows the cumulative silicon damage as a percentage and it is indicated by the secondary axis on the right side of the plot. 


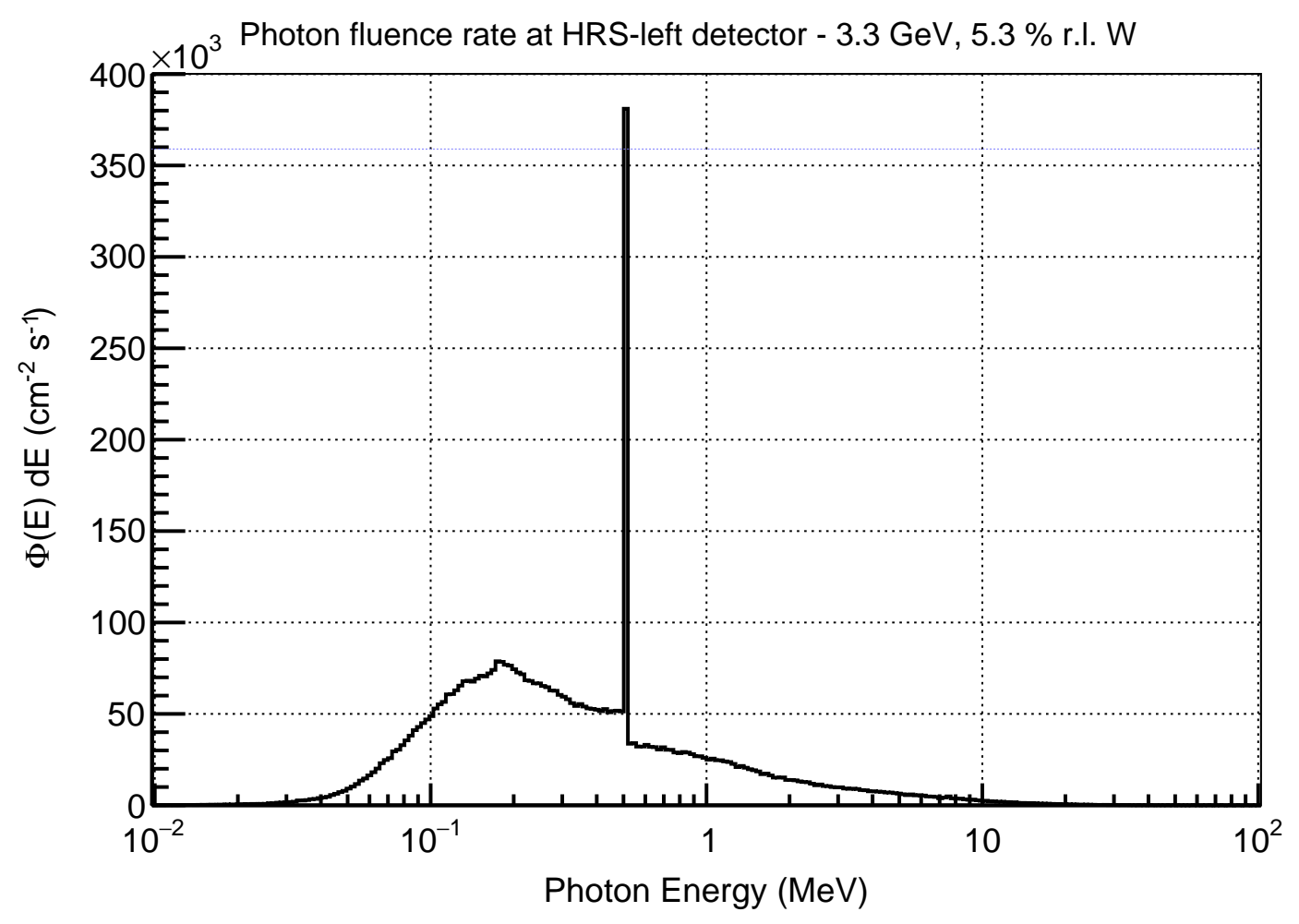

Figure 3.26: Shown is the simulated photon fluence-rate spectrum at the HRS control electronics for the APEX experiment with the $3.3 \mathrm{GeV}$ setting. Beam current was assumed to be $120 \mu \mathrm{A}$. The histogram has equal-width bins in the log scale and the fluence is per energy bin which is indicated by $\Phi(\mathrm{E}) \mathrm{dE}$. 
Table 3.3: This table summarizes the calculation of $1 \mathrm{MeV}$ neutron equivalent fluence at HRS control electronics for PREX-I, RCS and APEX experiments.

\begin{tabular}{|c|c|c|c|c|}
\hline Experiment & Days & $\begin{array}{l}\text { Radiation } \\
\text { type }\end{array}$ & $\begin{array}{c}1 \mathrm{MeV} \\
\text { neutron } \\
\text { equivalent } \\
\text { fluence rate } \\
\left(\mathrm{n}_{\mathrm{eq}} \mathrm{cm}^{-2} \mathrm{~s}^{-1}\right)\end{array}$ & $\begin{array}{l}\text { Total } \\
1 \mathrm{MeV} \\
\text { neutron } \\
\text { fluence } \\
\left(\mathrm{n}_{\mathrm{eq}} \mathrm{cm}^{-2}\right)\end{array}$ \\
\hline $\begin{array}{l}\text { PREX-I, } 1.06 \mathrm{GeV} \\
70 \mu \mathrm{A} 10 \% \text { r.l. } \mathrm{Pb}\end{array}$ & $\begin{array}{l}16 \text { effective } \\
\text { days }\end{array}$ & $\begin{array}{c}e^{+} / e^{-} \\
\text {neutrons }\end{array}$ & $\begin{array}{l}6.2 \mathrm{E}+04 \\
2.0 \mathrm{E}+05\end{array}$ & $\begin{array}{l}8.6 \mathrm{E}+10 \\
2.7 \mathrm{E}+11\end{array}$ \\
\hline $\begin{array}{c}\mathrm{RCS}, 3.481 \mathrm{GeV} \\
40 \mu \mathrm{A}\end{array}$ & $\begin{array}{l}16 \text { effective } \\
\text { days }\end{array}$ & $\begin{array}{c}e^{+} / e^{-} \\
\text {neutrons }\end{array}$ & $\begin{array}{l}1.8 \mathrm{E}+03 \\
5.1 \mathrm{E}+03\end{array}$ & $\begin{array}{l}2.5 \mathrm{E}+09 \\
7.0 \mathrm{E}+09\end{array}$ \\
\hline $\begin{array}{l}\text { APEX, } 1.1 \mathrm{GeV} \\
50 \mu \mathrm{A} 0.7 \% \text { r.l. C }\end{array}$ & 6 PAC days & $\begin{array}{c}e^{+} / e^{-} \\
\text {neutrons }\end{array}$ & $\begin{array}{l}5.7 \mathrm{E}+02 \\
1.7 \mathrm{E}+03\end{array}$ & $\begin{array}{l}3.0 \mathrm{E}+08 \\
8.9 \mathrm{E}+08\end{array}$ \\
\hline $\begin{array}{c}\text { APEX, } 2.2 \mathrm{GeV} \\
100 \mu \mathrm{A} 2.8 \% \text { r.l. W }\end{array}$ & 6 PAC days & $\begin{array}{c}e^{+} / e^{-} \\
\text {neutrons }\end{array}$ & $\begin{array}{l}3.4 \mathrm{E}+03 \\
1.3 \mathrm{E}+04\end{array}$ & $\begin{array}{l}1.8 \mathrm{E}+09 \\
6.6 \mathrm{E}+09\end{array}$ \\
\hline $\begin{array}{c}\text { APEX } 3.3 \mathrm{GeV} \\
120 \mu \mathrm{A} 5.3 \% \text { r.l. W }\end{array}$ & 6 PAC days & $\begin{array}{c}e^{+} / e^{-} \\
\text {neutrons }\end{array}$ & $\begin{array}{l}5.9 \mathrm{E}+03 \\
2.0 \mathrm{E}+04\end{array}$ & $\begin{array}{l}3.1 \mathrm{E}+09 \\
1.1 \mathrm{E}+10\end{array}$ \\
\hline $\begin{array}{l}\text { APEX } 4.4 \mathrm{GeV} \\
90 \mu \mathrm{A} 5.3 \% \text { r.l. W }\end{array}$ & 12 PAC days & $\begin{array}{c}e^{+} / e^{-} \\
\text {neutrons }\end{array}$ & $\begin{array}{l}3.2 \mathrm{E}+03 \\
1.2 \mathrm{E}+04\end{array}$ & $\begin{array}{l}3.3 \mathrm{E}+09 \\
1.3 \mathrm{E}+10\end{array}$ \\
\hline
\end{tabular}


the HRS control electronics are less than the ESAD threshold value. But, the $1 \mathrm{MeV}$ fluence can exceed the ESAD threshold in certain locations in the JLab Hall A. It is important to have an understanding of where in Hall A this ESAD threshold is exceeded. We evaluated $1 \mathrm{MeV}$ equivalent neutron fluence at several other key locations in Hall A. Apart from the HRS control electronics area, we calculated fluence about $16 \mathrm{~m}$ upstream of the target, $1 \mathrm{~m}$ above the target where the motor that controls the target will be located and $1 \mathrm{~m}$ to the side of the target chamber where the motor that controls the SciFi detector will be located. We will discuss the SciFi detector in detail in Chapter 4. Table 3.4 summarizes the calculated $1 \mathrm{MeV}$ neutron fluences at all four locations. Figure 3.27 shows a side view and a top view of the Geant4 model of the APEX experiment as well as the four Geant4 scoring volumes (labeled in the figure) at the four different locations. Most of the neutrons are produced at the target. Assuming the target is a point neutron source, the radiation follows the inversesquare law and taking the radiation level at $1 \mathrm{~m}$ distance $\left(\sim 1.5 \mathrm{E}+13 \mathrm{n}_{\mathrm{eq}} \mathrm{cm}^{-2}\right)$ as a reference, we can predict radiation levels at any location in the hall. Based on these assumptions even at an arbitrary distance of $2 \mathrm{~m}$ the radiation level was already below the ESAD threshold. The calculated radiation levels at the location of HRS electronics ( $\sim 20 \mathrm{~m}$ from the target) shown in Table 3.4 agreed with our predictions.

Ultimately, we focused our Geant4 simulations the PREX-I and APEX experiments. We demonstrated that at the location of HRS control electronics, the radiation levels expected for APEX experiment are 8.9 times lower compared to PREX-I experiment. During PREX-I the experiment was down $50 \%-70 \%$ of the time. There were many reasons that contributed to this down time, but if we assume that the down time came only from radiation induced electronics problems, we predict that APEX will have only $\sim 8 \%$ down time. PREX-I used a Lead target which partially melted during the experiment and there were issues with vacuum leaks due to rubber 
Table 3.4: Table summarizes the calculation of $1 \mathrm{MeV}$ neutron equivalent fluence at different locations in the hall A during APEX experiment. The second column is the fluence only from neutrons and the thrid column is the fluence only from $e^{+} / e^{-}$. All the proposed APEX settings are included in this calculation.

\begin{tabular}{|c|c|c|}
\hline Location & $\begin{array}{c}1 \mathrm{MeV} \text { neutron } \\
\text { equivalent fluence } \\
\left(\begin{array}{c}\text { Only from neutrons }) \\
\left(\mathrm{n}_{\mathrm{eq}} \mathrm{cm}^{-2}\right)\end{array}\right.\end{array}$ & $\begin{array}{c}1 \mathrm{MeV} \text { neutron } \\
\text { equivalent fluence } \\
\left(\text { Only from } e^{+} / e^{-}\right) \\
\left(\mathrm{n}_{\mathrm{eq}} \mathrm{cm}^{-2}\right)\end{array}$ \\
\hline HRS electronics area & $3.1 \mathrm{E}+10$ & $8.5 \mathrm{E}+09$ \\
\hline Upstream & $1.9 \mathrm{E}+10$ & $1.7 \mathrm{E}+08$ \\
\hline $\begin{array}{c}\text { Target motion motor } \\
(1 \mathrm{~m} \text { from beam })\end{array}$ & $1.6 \mathrm{E}+13$ & $1.7 \mathrm{E}+13$ \\
\hline $\begin{array}{c}\text { SciFi motion motor } \\
(1 \mathrm{~m} \text { from beam })\end{array}$ & $1.5 \mathrm{E}+13$ & $2.2 \mathrm{E}+12$ \\
\hline
\end{tabular}




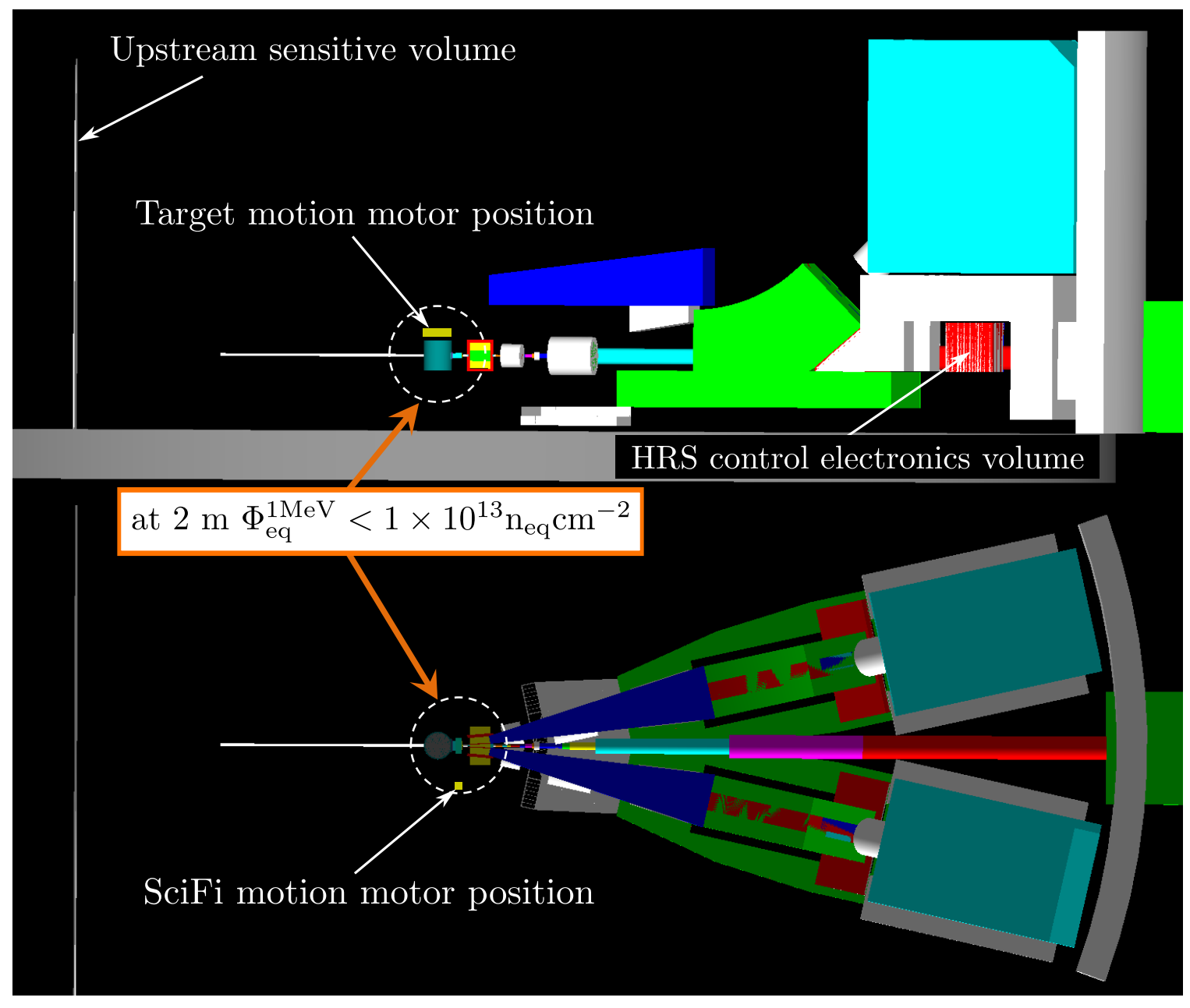

Figure 3.27: A side view (top) and a top view (bottom) of the APEX simulation setup are shown. Four Geant4 scoring volumes were placed in the hall A at four different locations (labeled in the figure) to evaluate radiation levels in terms of $1 \mathrm{MeV}$ equivalent neutron fluence. The circle with a dashed line indicates an arbitrary distance of $2 \mathrm{~m}$. For the APEX experiment, the radiation levels are already below the ESAD threshold at this distance. 
O-rings failing. On the other hand, APEX uses a robust Tungsten target which will not melt and it uses metal O-rings on vacuum pipes closer to the target instead of rubber O-rings. This will definitely reduce the down time even further.

\subsection{Absolute comparisons of our Geant4 simula- tions}

In the previous section we presented a relative comparison of radiation levels, calculated using Geant4 simulations, for different experiments in JLab Hall A. In this section, we compare our simulation results with actual data. The first measurement with which we compare our simulation involved a study of the increase of dark current in Silicon photomultipliers (SiPM). The study is described by Qiang et al. [63], and

when cast as an equivalent fluence of $1 \mathrm{MeV}$ neutrons, the author saw indication of radiation levels about $30 \%$ lower than our simulation. The second measurement with which we compare our simulation involved a neutron dose rate measurement using a neutron detector known as a "SNOOPY" (to be discussed more shortly). Naively, our simulations showed significantly higher radiation levels than were measured with the SNOOPY's. We will argue, however, that a number of factors, including dead-time, may potentially explain most of the discrepancy. Indeed, we will argue that care needs to be taken when using SNOOPY's in particularly high radiation fields.

\subsubsection{Comparison with silicon damage measurements}

A group at JLab performed an experiment to evaluate the radiation tolerance of Silicon photomultipliers (SiPM) [63]. A SiPM is a solid-state single-photon-sensitive device built from an avalanche photodiode array on a silicon substrate. Radiation can 
alter properties of SiPM devices, especially neutron radiation. In the test described in Ref. [63], two SiPM devices were studied in JLab Hall A during the PREX-I experiment. The SiPM units were placed about $16 \mathrm{~m}$ upstream of the PREX-I target and the damage to the SiPM units was evaluated by measuring the change in the dark current. Subsequently, they irradiated two different SiPM units, the same as the ones they studied in hall A, using a calibrated Am/Be neutron source. During the later studies using the calibrated neutron source, they irradiated the two SiPM units until the change in the dark current was the same as the change in the dark current during the studies in JLab Hall A. The study using the calibrated neutron source provided a measure of SiPM damage due to a known neutron fluence. Using the comparison, the authors concluded that, at the location where they performed their studies, the radiation was the equivalent of a $1 \mathrm{MeV}$ neutron fluence of $3.7 \times 10^{9} \mathrm{n}_{\text {eq }} / \mathrm{cm}^{2}$.

Figure 3.28 shows the simulated neutron spectrum in the upstream region of JLab Hall A. Convolution of this neutron spectrum with the Silicon damage curve shown in Figure 3.20 gives us the silicon damage in terms of a rate of equivalent $1 \mathrm{MeV}$ neutron fluence. For a $50 \mu \mathrm{A}$ electron beam, our PREX-I simulation predicted a silicon damage rate of $4.6 \times 10^{4} \mathrm{n}_{\mathrm{eq}} \mathrm{cm}^{-2} \mathrm{~s}^{-1}$ in the upstream region of the experimental hall. To compare with the aforementioned studies using SiPM's, we must integrate this damage rate over 32.5 hours, the time during which the SiPM's were irradiated in Hall A. Our Geant 4 simulation thus gives a total equivalent fluence of $1 \mathrm{MeV}$ neutrons of $5.4 \times 10^{9} \mathrm{n}_{\mathrm{eq}} \mathrm{cm}^{-2}$. The SiPM result is around $30 \%$ below our simulation, which is reasonable agreement. A similar comparison was done between SiPM results and Geant3 calculations performed by Pavel Degtiarenko of the JLab RadCon group who noted a similar level of agreement [63][64]. 


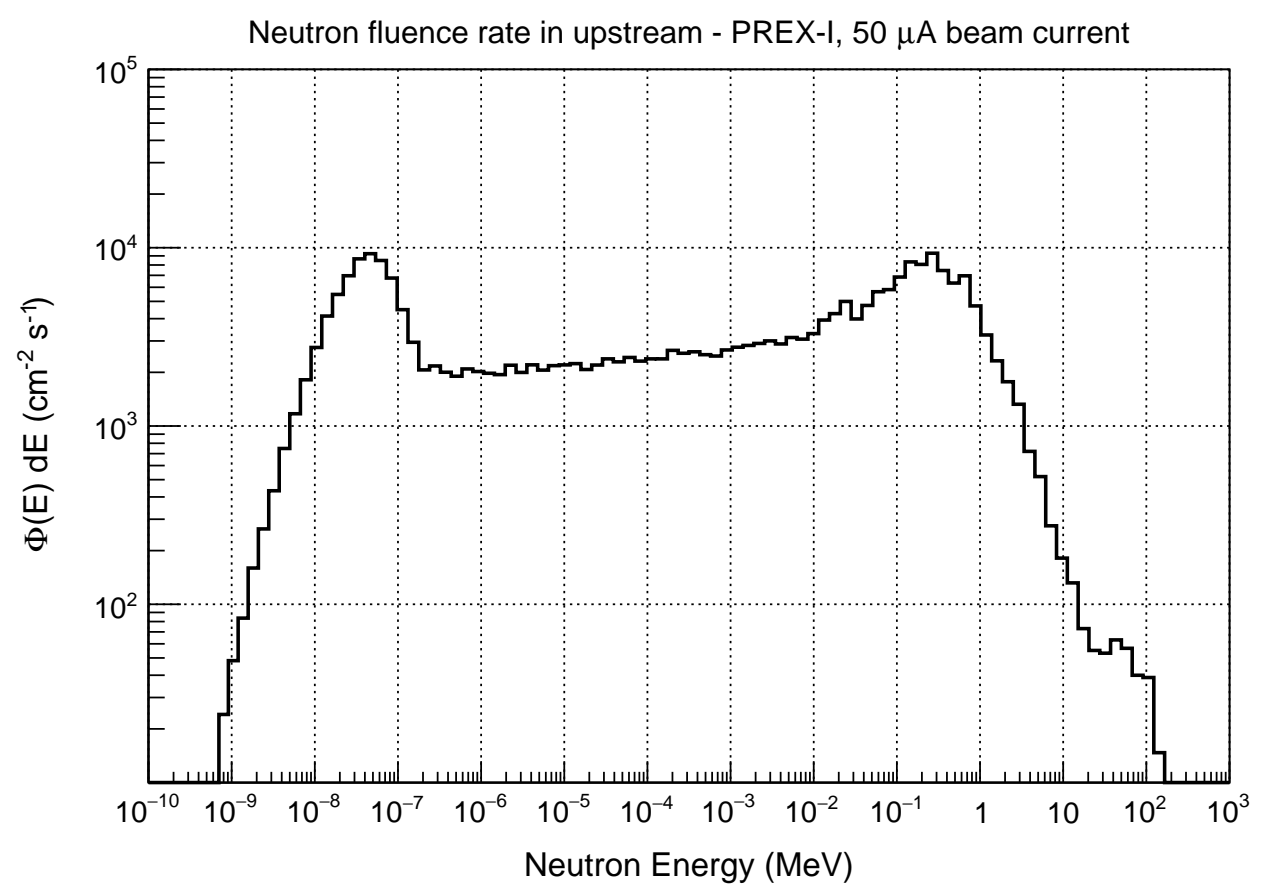

Figure 3.28: The simulated neutron spectrum in upstream region of Hall A for the APEX experiment. Events were binned by neutron energy. The histogram has equal-width bins in the log scale and the fluence is per energy bin which is indicated by $\Phi(\mathrm{E}) \mathrm{dE}$. We considered a beam current of $50 \mu \mathrm{A}$ for the calculation. 


\subsubsection{Comparison with neutron dose rate measurements}

The International Commission on Radiological Units and Measurements (ICRU) has introduced and defined the ambient dose equivalent, $H^{*}(10)$, as the operational quan-

tity for area monitoring [44]. The unit of ambient dose is the rem or the Sievert (Sv) and it provides an acceptable approximation to the dosimetric quantities which are used as measures of radiation damage to the human body. Here we will focus on neutron radiation. Figure 3.29 shows a plot of neutron ambient dose conversion coefficients published by International Atomic Energy Agency (IAEA) which can be used to calculate the neutron ambient dose equivalent from a known neutron fluence spectrum [49]. The total dose can be computed as

$$
\mathrm{rem}=\int_{0}^{\infty} \frac{d \Phi}{d E} \beta(E) d E
$$

where $d \Phi / d E$ is the differential neutron fluence and $\beta(E)$ is the fluence-to-dose conversion coefficients shown in Figure 3.29.

Neutron detectors which have the capability to measure neutron dose in the units of rems are often called "rem-meters". The radiation control department (RadCon) at JLab uses a rem-meter manufactured by Canberra Industries, model NP100, for area monitoring. For historical reasons that date back to World War II, such meters are also known as a "SNOOPY", apparently a code word chosen so that its meaning would not be overly obvious. SNOOPY's, can be used to measure the neutron dose rates in the units of rem/hr. A SNOOPY has a thermal neutron detector surrounded by moderating material in the shape of a cylinder. The NP100 uses a proportional counter filled with a gas, either ${ }^{3} \mathrm{He}(\mathrm{NP} 100 \mathrm{H})$ or $\mathrm{BF}_{3}(\mathrm{NP} 100 \mathrm{~B})$. The moderator of the SNOOPY is specially designed, so that the response function of the instrument reproduces the curve of the conversion coefficients associated with (Figure 3.29) 


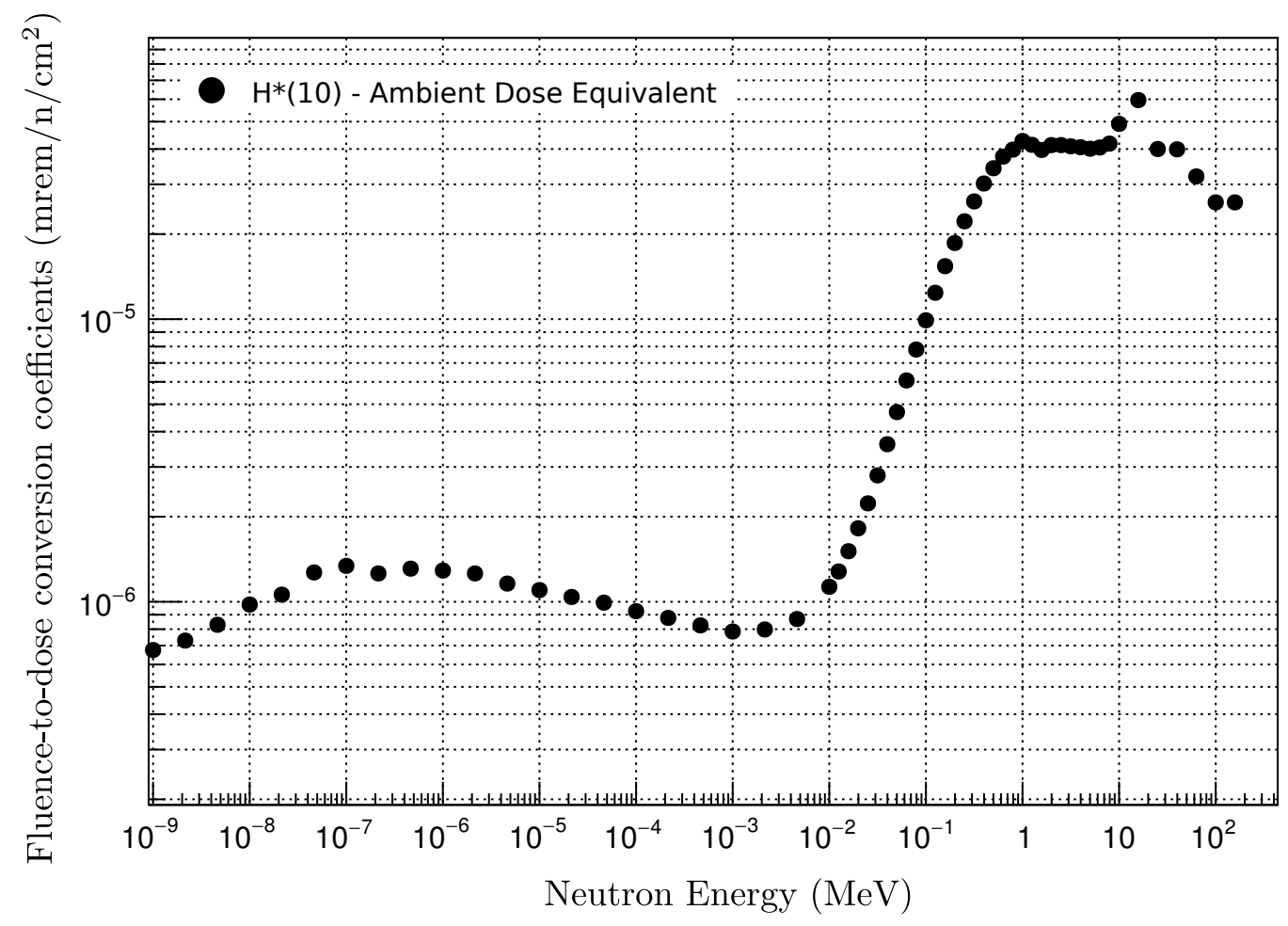

Figure 3.29: This plot shows the conversion coefficients for neutron fluence to neutron dose as a function of neutron energy. 
fluence-to-dose conversion curve. This idea was first developed by I.O. Andersson and J. Braun in 1964 [48]. A SNOOPY is sensitive to neutrons from thermal energies up to about $15 \mathrm{MeV}$.

A SNOOPY (using $\mathrm{BF}_{3}$ in the proportional chamber) was used to measure the neutron dose rates in JLab Hall A during the PREX-I and the RCS experiments. In both experiments the SNOOPY was placed in the upstream region of the experimental hall, close to the Hall A entrance, as shown in Figure 3.30. Figure 3.31 and Figure 3.32 show the measured neutron dose rates in the upstream region of Hall A during PREX-I and RCS respectively. These neutron dose rates were measured with different electron beam currents and are shown in Figure 3.31 and Figure 3.32 normalized to a $100 \mu \mathrm{A}$ beam for comparison.

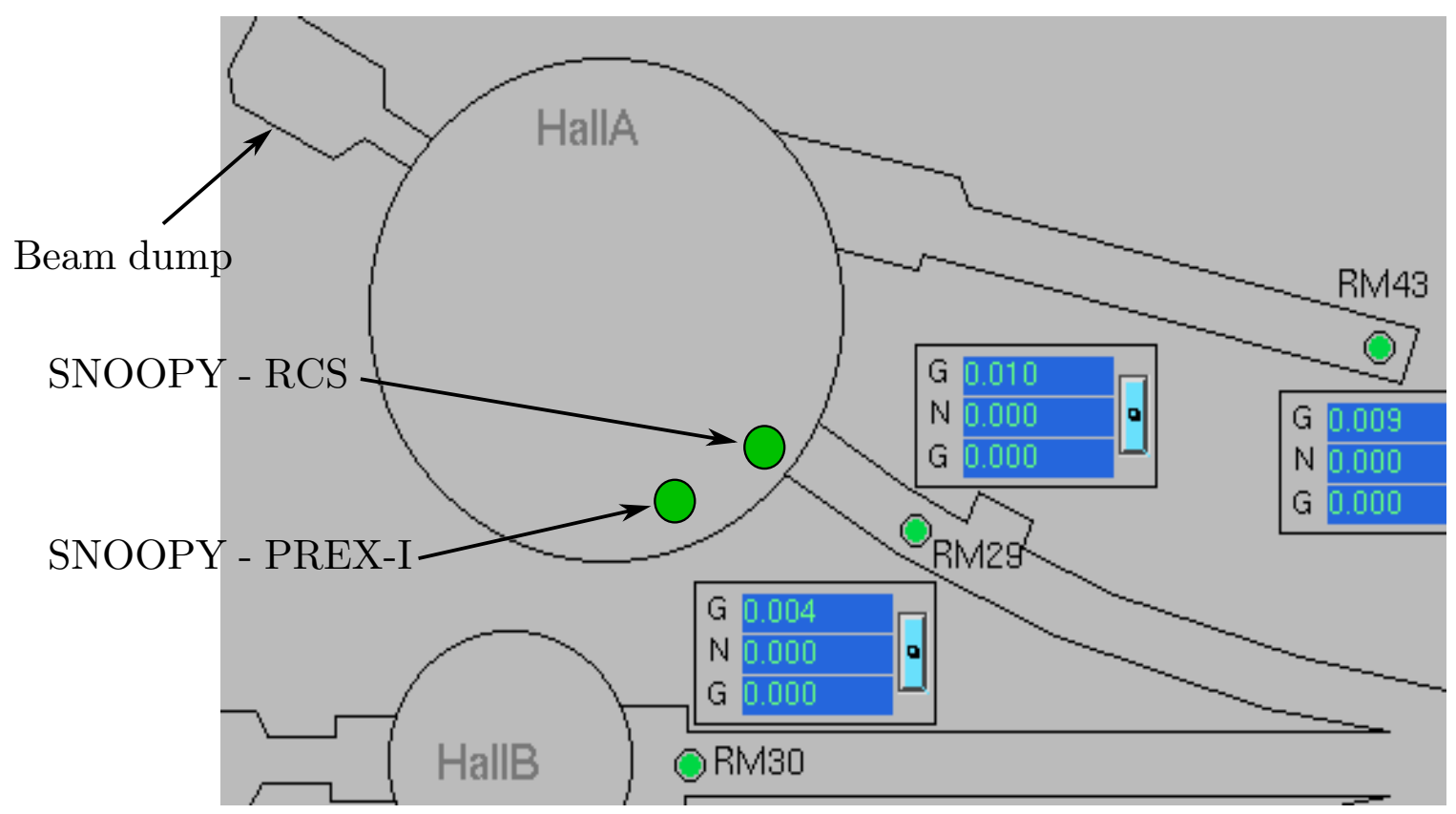

Figure 3.30: This diagram shows the positioning of NP100B (SNOOPY) in Hall A during the PREX-I and RCS experiments. In both cases SNOOPY's were placed in upstream region close to the entrance of Hall A. 
Figure 3.28 and Figure 3.33 show the simulated neutron fluence spectra in the upstream region of Hall A, for the PREX-I and the RCS experiments respectively. Equation 3.3 can be used to calculate the ambient dose equivalent. Here we make the assumption that the response of the calibrated SNOOPY reproduces reasonably well the fluence-to-ambient dose conversion curve shown in Figure 3.29. For PREX-I, the Geant4 simulation accordingly predicts a radiation level of $15 \mathrm{rem} / \mathrm{hr}$ for a $100 \mu \mathrm{A}$ beam. This is a factor of 7.2 larger than the measurements with the SNOOPY (see Figure 3.31). For RCS, the Geant4 simulation accordingly predicts a radiation level of $1.28 \mathrm{rem} / \mathrm{hr}$ for a $100 \mu \mathrm{A}$ beam. This is a factor of 2.9 larger than the measurements with the SNOOPY (see Figure 3.32).

While it is not reasonable to expect Geant4 to exactly reproduce the radiation field for a given experiment, the size of the disagreement discussed above raises questions concerning the source(s) of discrepancies. We discuss next potential problems associated with the response curve of the SNOOPY and possible dead-time issues.

The response curve of the SNOOPY

Any detector, including a SNOOPY, can be characterized by a response curve, which is given in counts per unit fluence (counts $/ \mathrm{n} / \mathrm{cm}^{2}$ ). We calculated the response curve of a SNOOPY using Geant4, which as discussed earlier, is designed to mimic the fluence-to-dose conversion curve. Figure 3.34 shows an exploded view of the Geant4 model of the SNOOPY used in our simulation. The calculation included 60 discrete energies with the assumption that neutrons incident on the SNOOPY were from a single horizontal direction.

Birattari and colleagues have studied a SNOOPY (Tracerlab model NP-1) using the Monte Carlo simulation software FLUKA [3] and have also validated their results with measurements [81]. In Figure 3.35, we reproduce a figure from their 
PREX-I neutron dose rate (mrem / hr) @ $100 \mu \mathrm{A}$

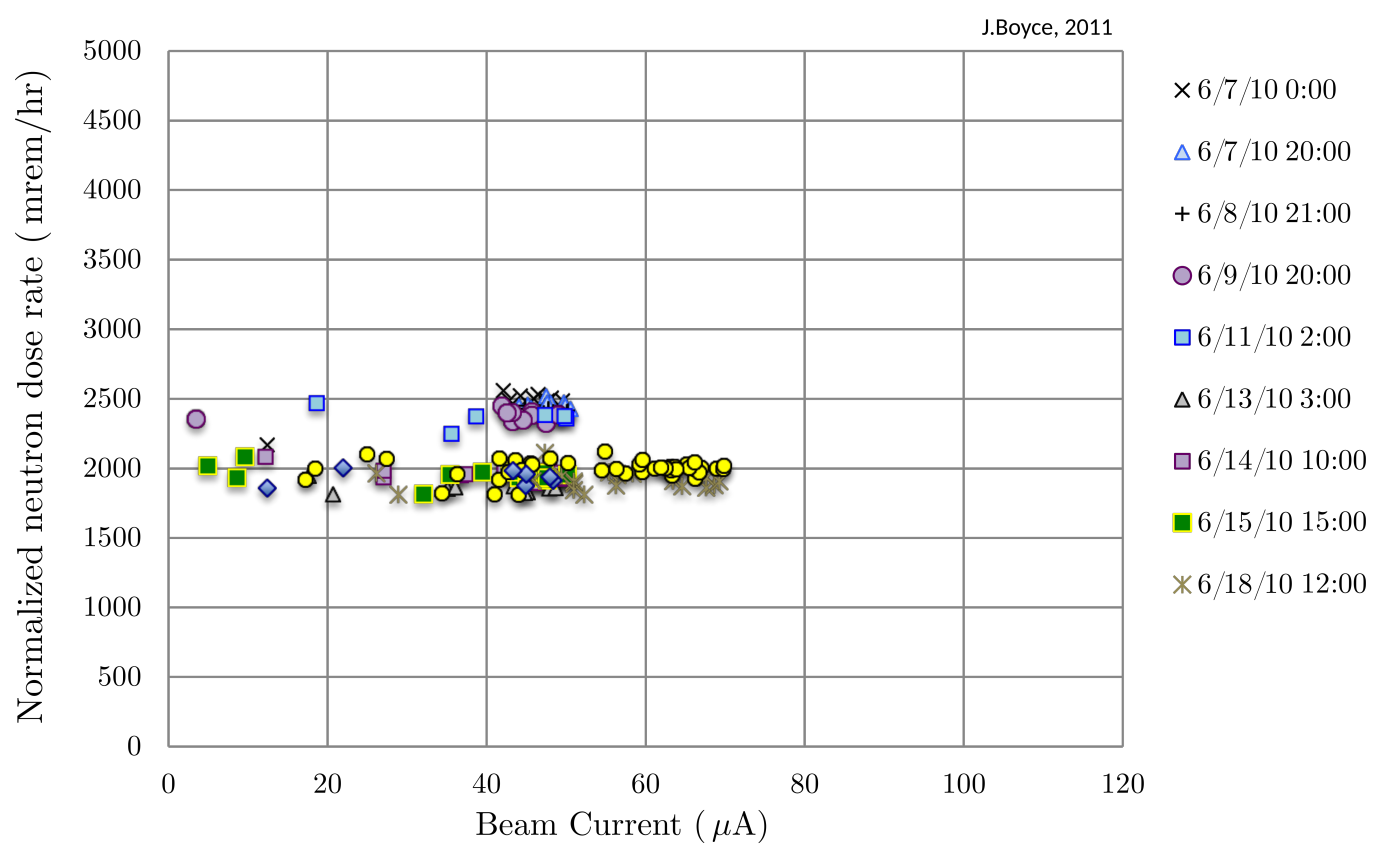

Average measured dose rate: $2080 \mathrm{mrem} / \mathrm{hr}$ at $100 \mu \mathrm{A}$

Geant 4 simulated dose rate: $15000 \mathrm{mrem} / \mathrm{hr}$ at $100 \mu \mathrm{A}$

Figure 3.31: Measured neutron dose rates in the upstream of the JLab Hall A using an NP100B neutron probe during the PREX-I experiment. Dose rates in the plot were measured at different beam currents and were normalized to a $100 \mu \mathrm{A}$ beam current. 
RCS neutron dose rate (mrem / hr) @ $100 \mu \mathrm{A}$

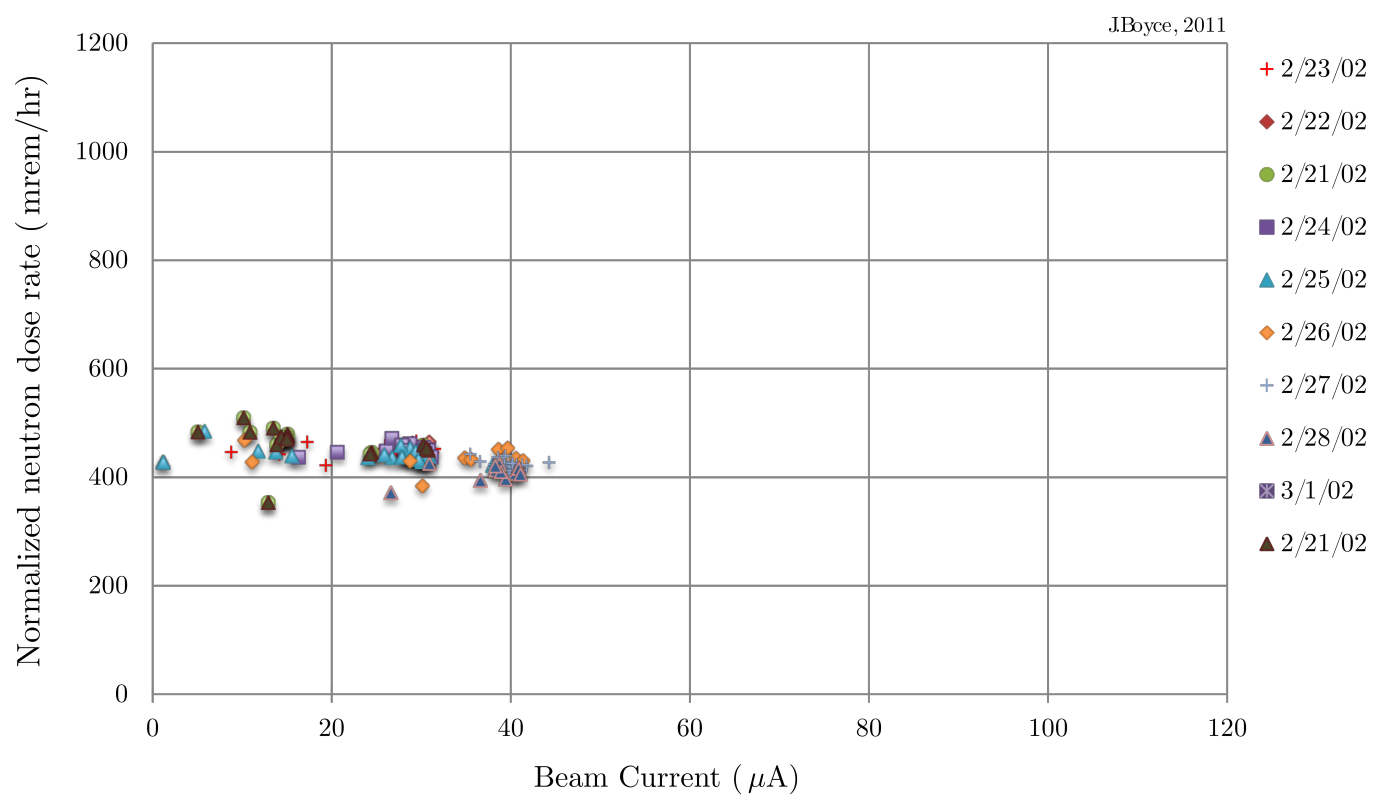

Average measured dose rate: $440 \mathrm{mrem} / \mathrm{hr}$ at $100 \mu \mathrm{A}$

Geant4 simulated dose rate: $1275 \mathrm{mrem} / \mathrm{hr}$ at $100 \mu \mathrm{A}$

Figure 3.32: Measured neutron dose rates in the upstream of the JLab Hall A using an NP100B neutron probe during the RCS experiment. Dose rates in the plot were measured at different beam currents and were normalized to a $100 \mu \mathrm{A}$ beam current. 


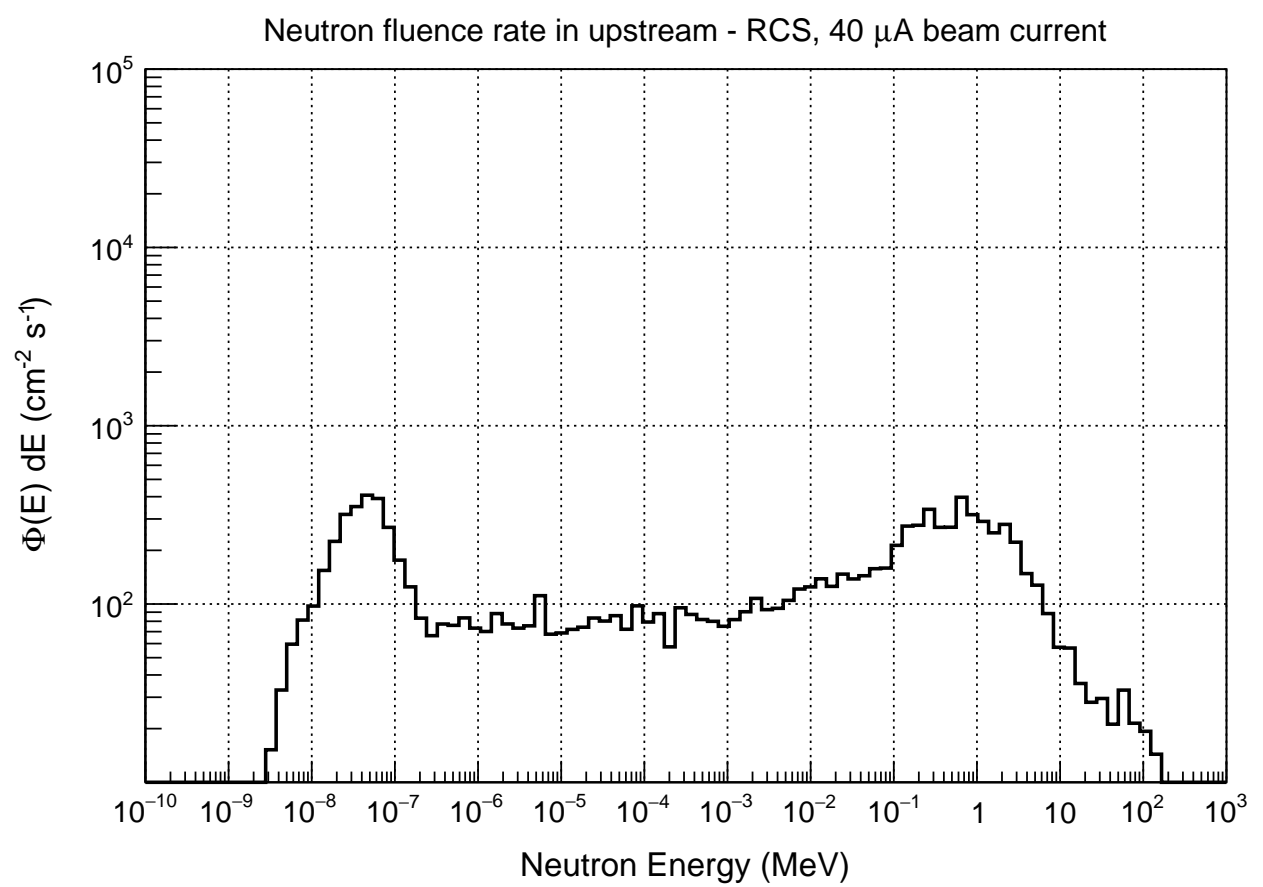

Figure 3.33: Simulated neutron fluence rates in the upstream of the JLab Hall A during the RCS experiment. We assumed a beam current of $40 \mu \mathrm{A}$. Events are binned by neutron energy. The histogram has equal-width bins in the log scale and the fluence is per energy bin which is indicated by $\Phi(\mathrm{E}) \mathrm{dE}$. 


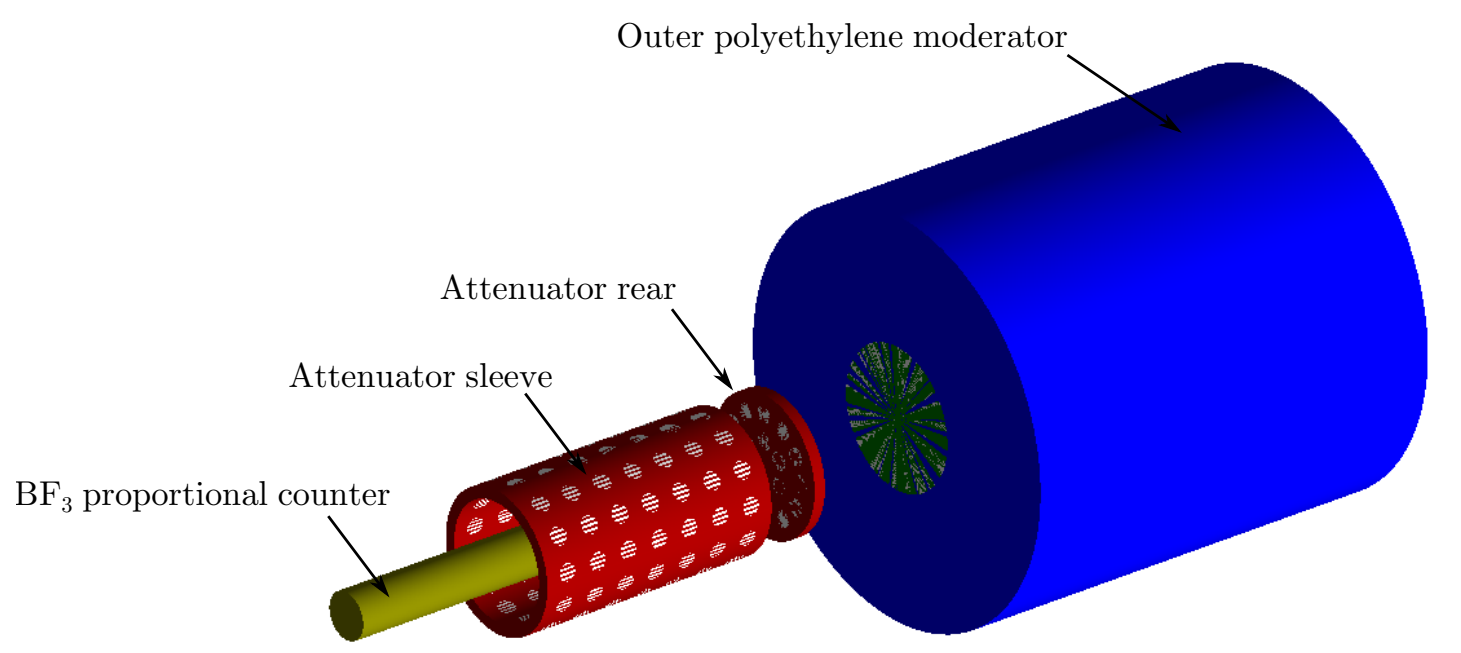

Figure 3.34: An exploded view of the SNOOPY. The outer polyethylene layer, Attenuators made of boron doped rubber and the proportional counter filled with $\mathrm{BF}_{3}$ gas are shown here.

paper in which the results from their calculation are shown with a black line with vertical bars at lower energies and open circles toward higher energies. While the energy dependence of Birattari's response curve follows some features of the $H^{*}(10)$ fluence-to-dose curve (shown with a solid black line) fairly well over some energies, there are clearly significant differences. Also shown in Figure 3.35 with open boxes (connected point-to-point using a red line) are the results of our Geant4 calculations. The agreement is excellent. With the agreement between our simulations and that of Birattari, and Birattari's experimental checks of his own results, we are confident that the response curve of Figure 3.35 provides a more accurate means for comparing our simulations with measurement than does the fluence-to-dose conversion curve in Figure 3.29. We note that the details of the SNOOPY geometry and material come from the work of Tessler et al. [50] and Mares et al. [51] and we also have excellent agreement with Tesseler. Complete details of the SNOOPY detector simulation can be found in Appendix A. 


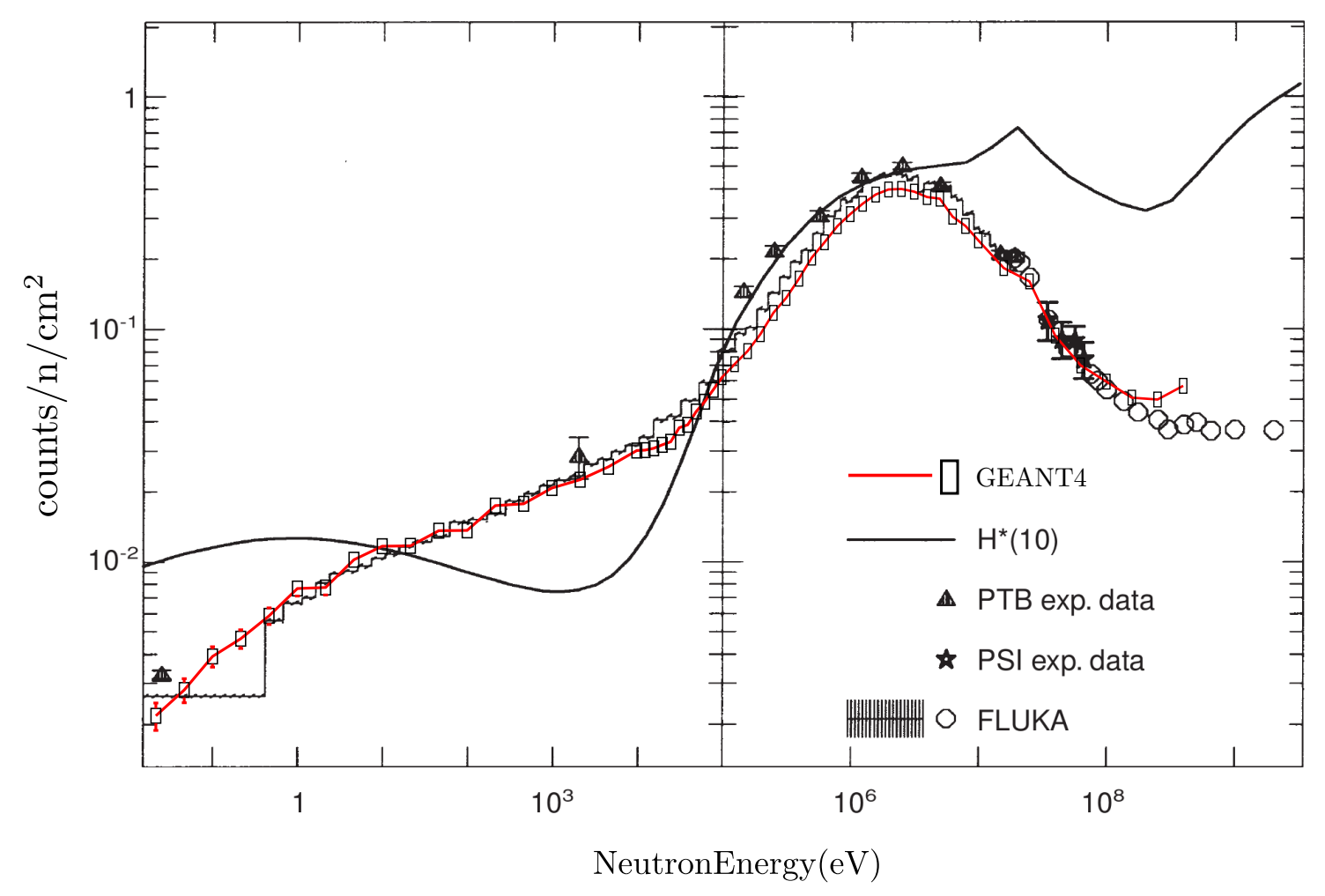

Figure 3.35: The original figure of this is from [81]. It compares the FLUKA calculation of the SNOOPY response to neutrons with measurements. The SNOOPY response calculated using Geant 4 in this study is plotted in the same figure for easy comparison.

Using our calculated response curve, we can predict the count rate of a SNOOPY for any situation. For PREX, a SNOOPY should register 28,800 counts/s in the upstream region in Hall A. For the Am/Be source that JLab uses for calibration, a SNOOPY should register 29 counts/s at a $1 \mathrm{~m}$ distance from the source. The aforementioned Am/Be source produces a radiation level of $13 \mathrm{mrem} / \mathrm{hr}$ according to a calibration that can be traced back to NIST. We would thus expect that the neutron radiation in the hall should be equal to $(28,800 / 29) 13 \mathrm{mrem} / \mathrm{hr}=12,910 \mathrm{mrem} / \mathrm{hr}$, whereas the SNOOPY's registered $2080 \mathrm{mrem} / \mathrm{hr}$, a factor of 6.2 difference. While still a large discrepancy, this is still smaller than the factor of 7.2 difference discussed 
earlier. We accordingly suggest that the response curve is responsible for part of the overall discrepancy. Note that in the above discussion, the overall efficiency of any specific device cancels out.

The dead-time of the SNOOPY

In detector systems, dead time is the minimum amount of time that must separate two events, in order them to be detected. Dead time can occur due to limitations either in the detector or the electronics. If the event rate is too high, there is always a probability that some of the true events will be lost. Two models of the detector dead time behavior have been in common usage: paralyzable and nonparalyzable response [67]. In a nonparalyzable system, a fixed dead time is assumed to follow a count in the detector. True events that occur during the dead time will not be recorded as counts. The measured count rate in a nonparalyzable system can be given by,

$$
m=\frac{n}{1+n \tau}
$$

where $m$ is the measured count rate, $n$ is the true event rate and the $\tau$ is the system dead time. In a paralyzable system, following an event, the dead time will also be extended making things even worse. The measured count rate in a paralyzable system is given by,

$$
m=n e^{-n \tau} .
$$

The manual from Canberra Industries for the model NP100, does not quote a deadtime. However, while there are not many published results to be found on the deadtime of the SNOOPY detector, Moyers and colleagues have found that the dead-time of a SNOOPY (NP-2) is $\sim 62.4 \mu$ s [68]. We note that the SNOOPY discussed in 
Moyers et al., the model NP2, is similar in all relative aspects to the NP100 used at JLab (this is one point that is worth further investigating). Earlier in this section we calculated the count rate in a SNOOPY based on our PREX-I simulation to be 28,800 counts/s. A dead-time of $\sim 62.4 \mu$ s would imply a loss rate of $65 \%$ in a nonparalyzable system and a loss rate of $83 \%$ in a paralyzable system. A similar calculation suggests that for RCS the SNOOPY should register a count rate of 2560 counts/s, with a loss rate of $\sim 15 \%$ for both nonparalyzable and paralyzable systems. All this suggests that the issue of dead-time is worth further study and may indeed be an important issue for high-radiation experiments.

\subsubsection{Summary of absolute comparisons}

As discussed earlier, a comparison of the absolute magnitude of the radiation level from our simulations (particularly those of PREX-I) with actual measurements naively seems to tell a complicated story. The simulations agree quite well with the (published) measurements that are based on studies of the dark current of SiPMs. In

contrast, however, the simulations predict much higher levels of radiation than a face-value interpretation of SNOOPY readings. The discussion in section 3.4.2, however, suggests there may be good reasons for that apparent disagreement. To see this, we need to be very careful defining what exactly we are comparing. In what follows, we ask the following question:

If we calibrate a SNOOPY with a specific well-defined calibration procedure, what do we expect the SNOOPY to read during different experimental situations?

The RadCon group uses an Am/Be neutron source with a calibration that can be traced to NIST. Furthermore, the radiation field at $1 \mathrm{~m}$ from the Am/Be source 
should be about $13 \mathrm{mrem} / \mathrm{hr}$ [70]. In what follows, we will use notation in which $S$ represents a measurement using a SNOOPY. Furthermore, $S(1 \mathrm{~m})$ will represent a measurement using the SNOOPY in which it is exposed to the aforementioned Am/Be source at a distance of one meter. Given the calibration of RadCon's Am/Be source, we can write:

$$
S(1 \mathrm{~m})=13 \mathrm{mrem} / \mathrm{hr}
$$

If we want to know what we would expect to measure in the hall, we can consider a quantity $\mathcal{S}^{H^{*} 10}$ which represents the calculated response of the neutron detector. Here the superscript $H^{*} 10$ signifies that when calculating the response, one assumes that the response function applied to the neutron spectrum has the shape of $H^{*}(10)$ curve shown in Figure 3.29. In the hall, the reading of the SNOOPY, S(hall), would thus be given by

$$
S(\text { hall })=\frac{\mathcal{S}^{H^{*} 10}(\text { hall })}{\mathcal{S}^{H^{*} 10}(1 \mathrm{~m})} 13 \mathrm{mrem} / \mathrm{hr} .
$$

In fact, however, as discussed earlier, the response function of a SNOOPY does not perfectly mimic $H^{*}(10)$. One can show that, for the case where the shape of the neutron spectrum in the hall is the same as the shape of the neutron spectrum of an Am/Be source, it would not matter whether or not the response function used, when calculating the expected reading of a SNOOPY, exactly mimics $H^{*}(10)$. In fact, if the neutron spectrum in the hall is simply proportional to the neutron spectrum of the Am/Be source, one can assume any response function and still correctly predict $S($ hall $)$. For the real case, however, where the neutron spectrum in the hall does not have the same shape as that of an Am/Be source, it is important to use the correct 
response function. The predicted measurement in the hall would then be given by

$$
S(\text { hall })=\frac{\mathcal{S}^{\text {true }}(\text { hall })}{\mathcal{S}^{\text {true }}(1 \mathrm{~m})} 13 \mathrm{mrem} / \mathrm{hr}
$$

where the superscript true signifies the actual response function of the SNOOPY. Indeed, in section 3.4.2, when the true response function was used, there was a smaller discrepancy between measurement and prediction. In fact, based on our previous discussion, one should also include a quantity $f^{\text {live }}$ that represents the fraction of neutrons that will be detected when dead time is taken into account. Depending on our assumptions, during PREX-I, at the location at which the neutron radiation was measured, we suggested that $0.17<f^{\text {live }}<0.35$.

Finally, as will be discussed in Chapter 5, there is also an effect that should be taken into account due to the fact that, during the calibration of the SNOOPY's, some radiation bounces off of the walls of the room in which the calibration is being performed. The net effect of this is that, when the SNOOPY is adjusted so that it reads $13 \mathrm{mrem} / \mathrm{hr}$, the actual radiation to which the SNOOPY was being exposed was larger. We believe these wall effects are already being accounted for during the SNOOPY calibration.

Including the dead-time correction factor, we have

$$
S(\text { hall })=\frac{\mathcal{S}^{\text {true }}(\text { hall }) f^{\text {live }}}{\mathcal{S}^{\text {true }}(1 \mathrm{~m})} 13 \mathrm{mrem} / \mathrm{hr}
$$

Table 3.5 summarizes the results of absolute comparisons. The second column shows the correction factor, $f^{\text {live }}$, that needs to be included in the calculation before we compare with SNOOPY measurements. The third column shows the SNOOPY measurements in the hall. The fourth column shows the expected reading of the SNOOPY calculated using equation 3.8 and the final column shows the expected 
Table 3.5: Summary of measured and predicted SNOOPY readings in JLab Hall A for the PREX-I and RCS experiments. All the measurements and calculations are assuming a $100 \mu \mathrm{A}$ beam current.

\begin{tabular}{|c|c|c|c|c|}
\hline \multicolumn{5}{|c|}{ Measured and predicted SNOOPY readings in the hall } \\
\hline Experiment & $f^{\text {live }}$ & $\begin{array}{c}\text { measured } \\
\text { (hall }) \\
(\text { mrem } / \mathrm{hr})\end{array}$ & $\begin{array}{c}\text { calculated } \\
S(\text { hall }) \\
(\mathrm{mrem} / \mathrm{hr})\end{array}$ & $\begin{array}{c}\text { corrected } \\
\text { (hall }) \\
(\mathrm{mrem} / \mathrm{hr})\end{array}$ \\
\hline PREX-I & $0.17<f^{\text {live }}<0.35$ & 2080 & 12910 & $(2195,4518)$ \\
\hline RCS & 0.85 & 440 & 1148 & 976 \\
\hline APEX $-1 \mathrm{GeV}$ & $0.95<f^{\text {live }}<0.96$ & - & 336 & $(319,322)$ \\
\hline APEX $-2 \mathrm{GeV}$ & $0.88<f^{\text {live }}<0.89$ & - & 853 & $(751,759)$ \\
\hline APEX $-3 \mathrm{GeV}$ & $0.82<f^{\text {live }}<0.83$ & - & 1459 & $(1196,1211)$ \\
\hline APEX $-4 \mathrm{GeV}$ & $0.84<f^{\text {live }}<0.85$ & - & 1227 & $(1031,1043)$ \\
\hline
\end{tabular}

SNOOPY reading calculated using equation 3.9 which includes the effects of deadtime. The calculated SNOOPY readings after corrections agree reasonably well with the measurements. The corrected value for PREX-I is at most factor of 2.2 higher than the measured value. The corrected value for RCS is also factor of 2.2 higher than the measured value. The level of agreement between SNOOPY measurements and calculations is now fairly comparable to the level of agreement in SiPM measurements and calculations. In Table 3.5, we have also predicted the SNOOPY readings we can expect during the APEX run. These calculations are normalized to a $100 \mu \mathrm{A}$ beam current and need to be scaled to the actual beam current during the APEX run for comparisons. Considering the complexity of the Hall A setup, the nature of the calculations and other potential sources of errors we do not expect a perfect agreement between the measurements and simulations. 


\subsection{Radiation sources and possible improvements in shielding}

In an experiment in Hall A, electrons that pass through the target can either interact with the target or reach the beam dump without any interaction. Some of the electrons that interact at the target can slightly diverge and hit beam line components. These interactions with the beam line can generate additional radiation sources apart from the primary radiation source, the target. Identifying radiation sources is important to decide on shielding strategies. We used Geant4 simulations to identify potential radiation sources on the beam line during the APEX experiment. Primary vertices - interaction points on the beam line after electrons pass through the target - were recreated using an algorithm. Figure 3.36 illustrates a drawing which describes the algorithm we used to identify track vertices. In the simulation, we used a $2 \mathrm{~m} \times 2 \mathrm{~m} \times 2 \mathrm{~m}$ polyethylene box as the Geant4 scoring volume at the HRS electronics area. The scoring volume at the left-HRS electronics area is labeled as HRS-L in Figure 3.36. Geant4 has the ability to save the history of particle tracks per each event. In our algorithm to reconstruct primary vertices, when a particle deposited energy in the HRS-L volume, we used the track histories to find the primary vertex. A particle at a primary vertex is not necessarily same as the particle that enters the HRS-L volume. Figure 3.36 shows three tracks which entered the HRS-L volume. For track A, the algorithm identifies the target itself or the point V1 as the primary vertex. In track $\mathrm{B}$, the primary vertex would be $\mathrm{V} 2$ and for track $\mathrm{C}$ it would be V3.

Figure 3.37 and Figure 3.38 illustrate how much power was deposited in the HRSL scoring volume due to $\gamma$ and neutrons respectively. The horizontal and the vertical axes of the plot indicate the $\mathrm{z}$ and $\mathrm{x}$ coordinates of the primary vertices respectively. The positive $\mathrm{z}$-axis in the hall is the beam direction, where $\mathrm{z}=0$ is the center of the 


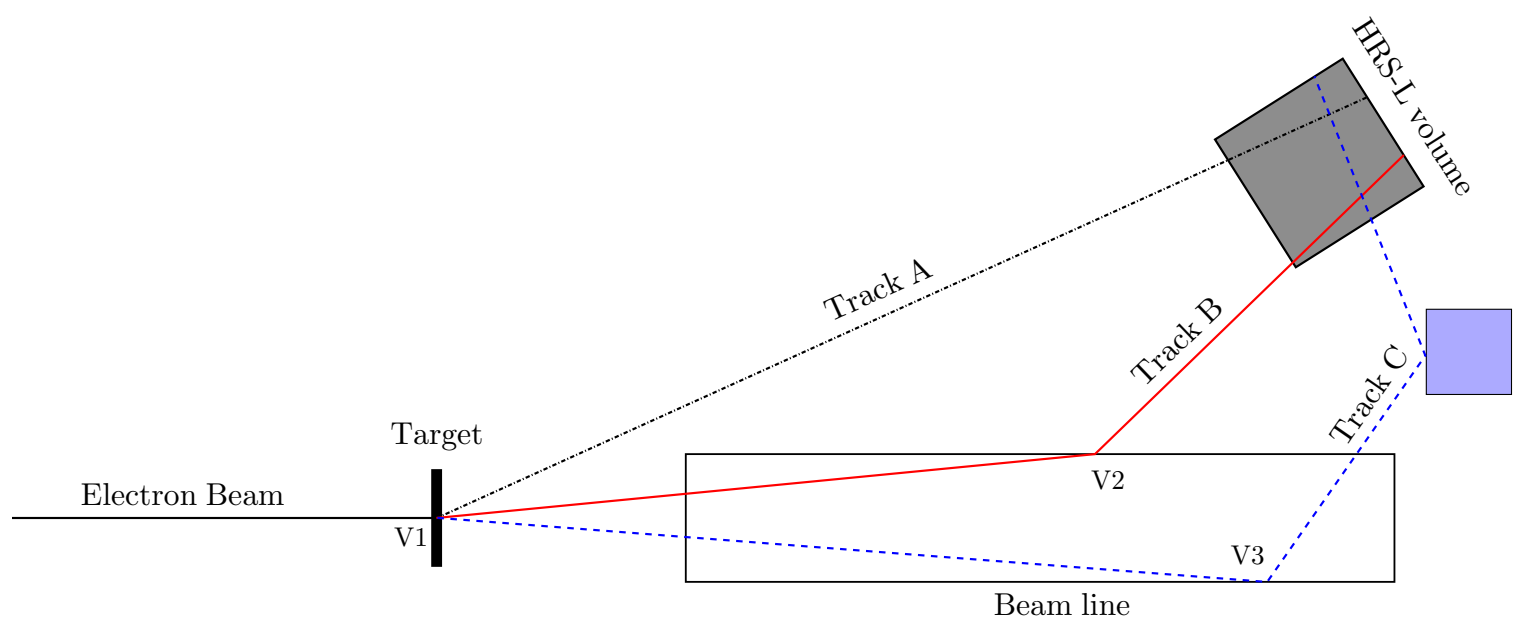

Figure 3.36: Identification of the primary vertices in the APEX experiment. When a particle track enters the HRS-L volume, it is tracked back to its primary vertex using an algorithm. The identified vertices of track A, B and C are V1, V2 and V3 respectively.

experimental hall and $\mathrm{z}=-105 \mathrm{~cm}$ is the target position. The $\mathrm{x}$-axis in the hall is transverse to the beamline and the vertical axis of the plot represents distance from the middle of the beamline in the $\mathrm{x}$ direction. Each pixel represents a particular point on the beamline, and its color encodes the deposition of power in the HRS$\mathrm{L}$ volume from events that originated at that point. Note that in Figure 3.37 the negative $\mathrm{x}$-values are not populated. This is because the tracks went into the HRS-L scoring volume mostly hit only the left side of the beam pipe. As can be seen in the Figure 3.37 and Figure 3.38, most of the power was deposited from the particle tracks with primary vertices (z coordinate) between 0 and $500 \mathrm{~cm}$. The 1D plot shown in Figure 3.39 confirms this fact quantitatively. The vertical axis of the plot in Figure 3.39 is the power deposited in the HRS-L volume and the horizontal axis is the $\mathrm{z}$ coordinate of the primary vertices. For Figure 3.39, we only considered vertices with $-100 \mathrm{~cm}<x<+100 \mathrm{~cm}$ which covers the beamline.

Figure 3.40 and Figure 3.41 illustrate blown-up views of the Figure 3.37 and the 


\section{Power deposited in HRS-L detector by $\gamma$ vertices}

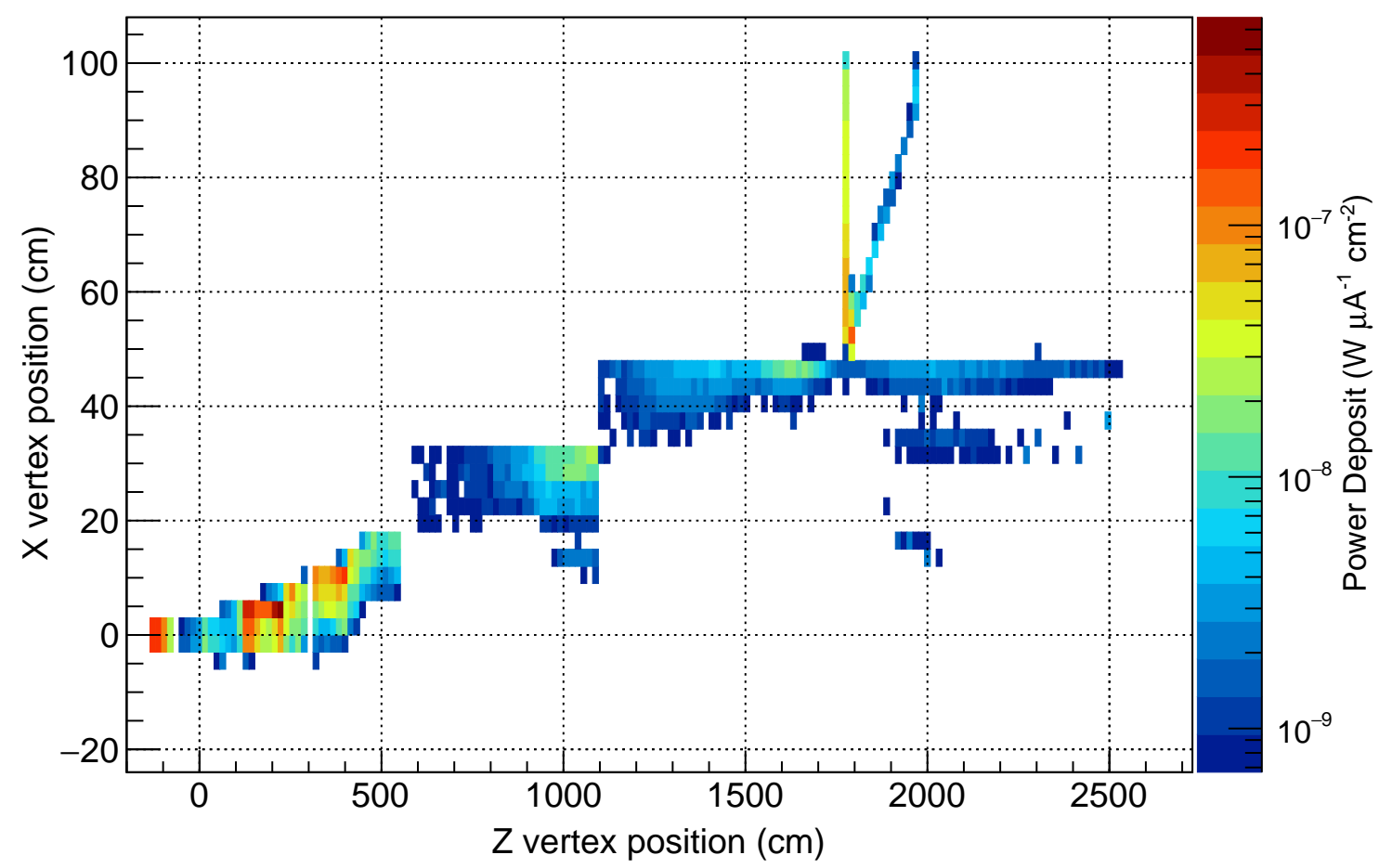

Figure 3.37: Simulated power deposition in the HRS-L volume due to $\gamma$ radiation for the APEX 3.3 GeV experiment. Each pixel in the 2D plot indicates a group of primary vertices ( $\mathrm{x}$ and $\mathrm{z}$ coordinates) and the color of a pixel represents how much energy was deposited in the HRS-L volume from the $\gamma$ rays originated from those vertices. 
Power deposited in HRS-L detector by neutron vertices

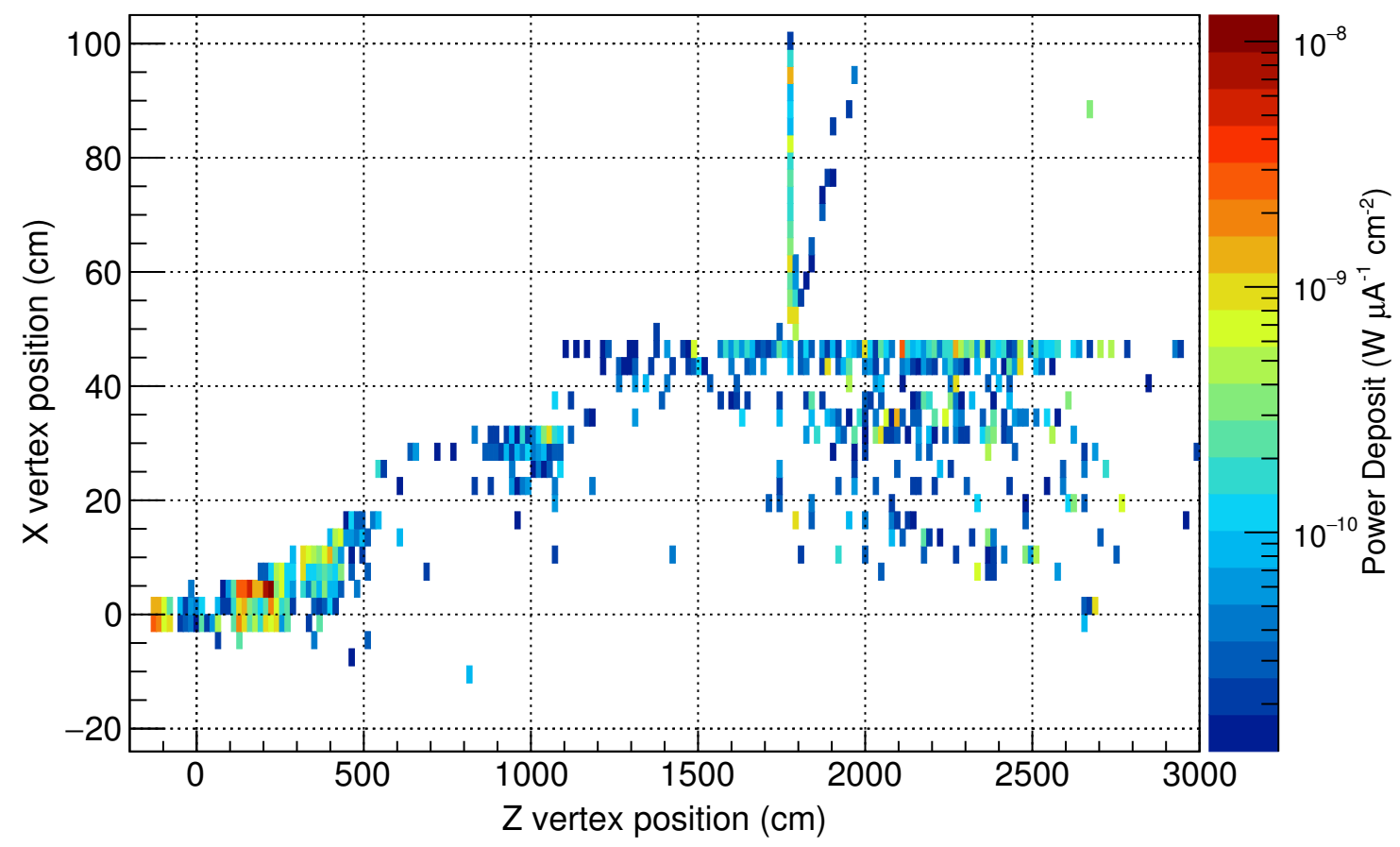

Figure 3.38: Simulated power deposition in the HRS-L volume due to neutrons for the APEX 3.3 GeV experiment. Each pixel in the 2D plot indicates a group of primary vertices ( $\mathrm{x}$ and $\mathrm{z}$ coordinates) and the color of a pixel represents how much energy was deposited in the HRS-L volume from the neutrons originated from those vertices. 


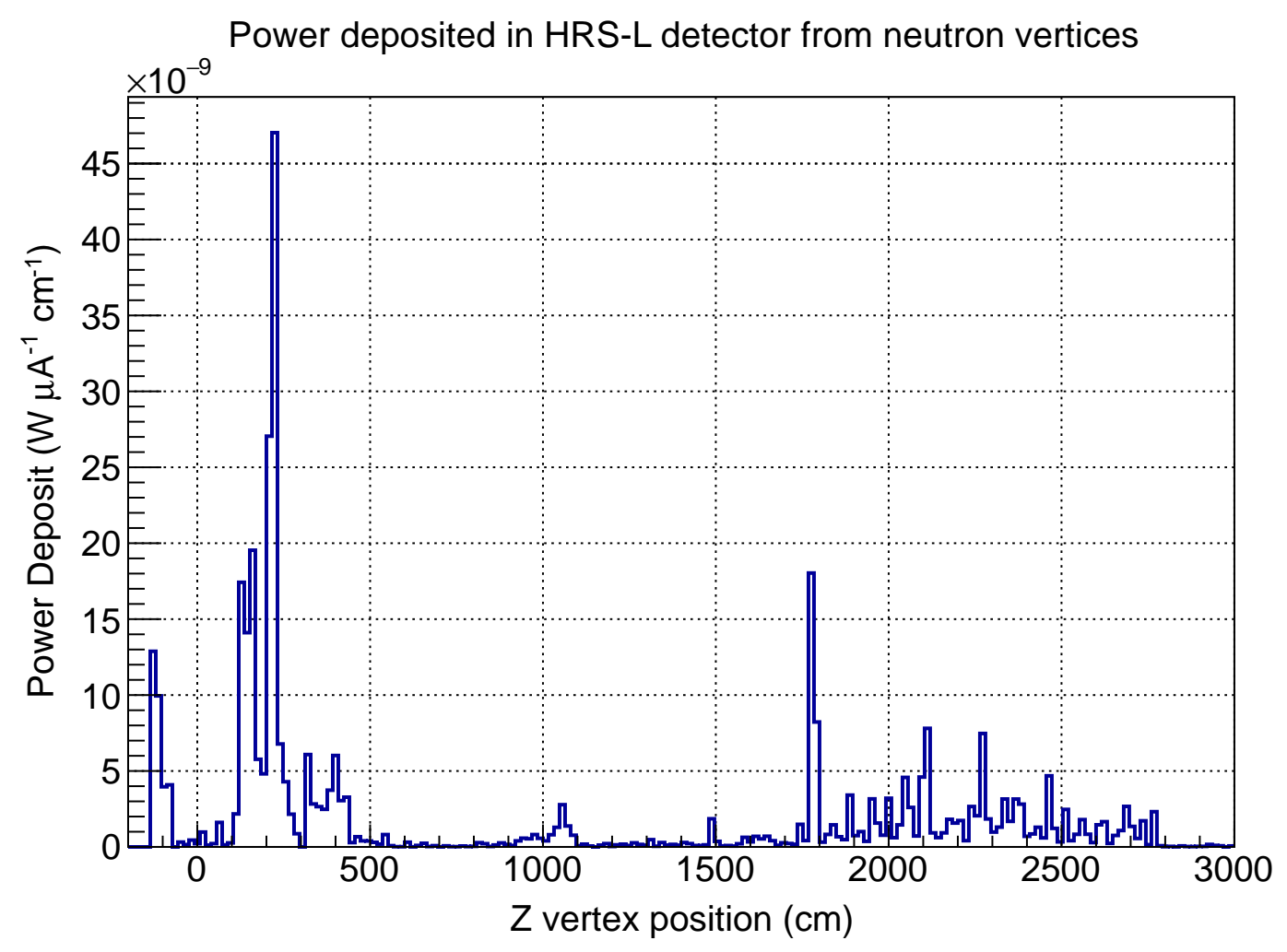

Figure 3.39: Simulated power deposition in the HRS-L volume due to neutrons for the APEX 3.3 GeV experiment. Y-axis shows the deposited power per each bin in the histogram and the $\mathrm{x}$-axis shows the $\mathrm{z}$ coordinate of a primary vertex. Events in the histogram were limited to the vertices with an $\mathrm{x}$ coordinate in the range $-100 \mathrm{~cm}<$ $\mathrm{x}<100 \mathrm{~cm}$. 
Figure 3.39 respectively. Only events that have primary vertices for which the $\mathrm{z}$ coordinate is less than $500 \mathrm{~cm}$ were included in the histograms. A drawing (top view) of the beam line is provided below each plot in which the horizontal axis of the drawing has been scaled to match the horizontal axis of the plot. The vertical axes of both the drawing and the plot correspond to the transverse position with respect to the beamline although the scales are different. For example in the drawing one can see the $1 \mathrm{~m}$ diameter scattering chamber centers at $z=-105 \mathrm{~cm}$ whereas the full range of the plot includes $-5 \mathrm{~cm} \leq \mathrm{x} \leq 15 \mathrm{~cm}$. Now we can further narrow the region of the beam line where most of the radiation is produced to $100 \mathrm{~cm}$ to $300 \mathrm{~cm}$ from the Hall A pivot. This region of the beam line, also called as the "telescoping beam pipe", has the smallest diameter of the entire downstream portion of the beamline. We can lower the radiation at the HRS electronics by shielding the telescoping beam pipe. Figure 3.42 shows a photograph of part of the telescoping beam pipe. As can be seen in the figure, there is very limited space available around the telescoping beam pipe for any additional shielding. Even though the results of the APEX simulations suggested that any additional shielding is not required to keep radiation at an acceptable level, we wanted to further study practical shielding methods. I will discuss the work we did on shielding studies in the next section.

We studied several methods to shield the HRS electronics area. One approach is to build a radiation shield closer to the HRS electronics using a neutron absorbing material such as polyethylene. This can help to lower the other types of radiation as well. We used Geant4 simulations to study the attenuation of radiation when it traverse polyethylene. Similar to the previous section, we used a $2 \mathrm{~m} \times 2 \mathrm{~m} \times 2 \mathrm{~m}$ Geant4 scoring volume (HRS-L scoring volume) with polyethylene at the HRS electronics. Figure 3.43 shows a top view of the left HRS with the HRS-L scoring volume. The HRS-L scoring volume was divided in to 40 layers in the $z^{\prime}$ direction and 10 lay- 


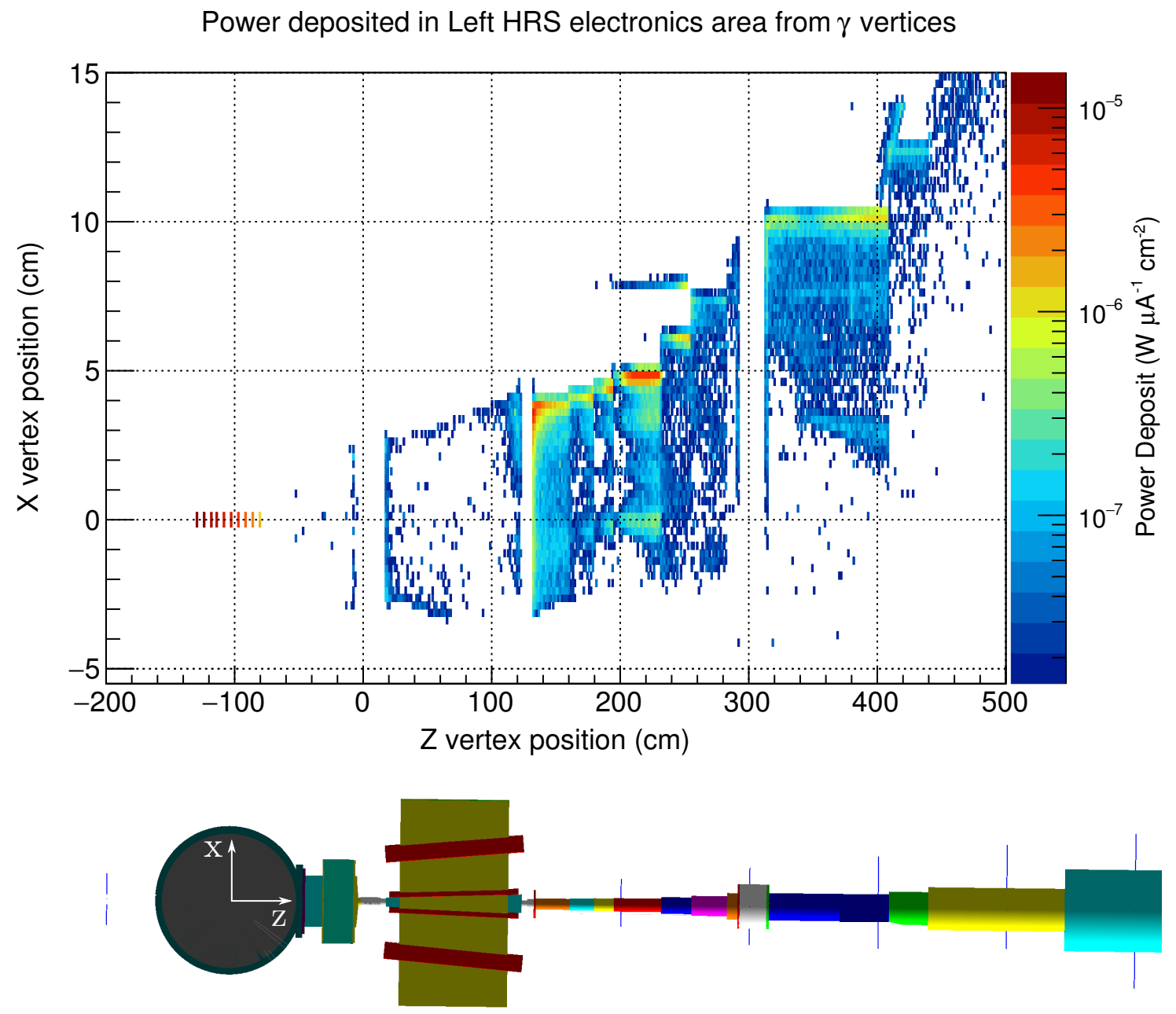

Figure 3.40: Simulated power deposition in the HRS-L volume due to $\gamma$ for the APEX $3.3 \mathrm{GeV}$ experiment. Each pixel in the 2D plot indicates a group of primary vertices ( $\mathrm{x}$ and $\mathrm{z}$ coordinates) and the color of the pixel represents how much energy was deposited in the HRS-L volume due to the $\gamma$ rays originated from those vertices. This is a blown-up view of the plot in Figure 3.37 


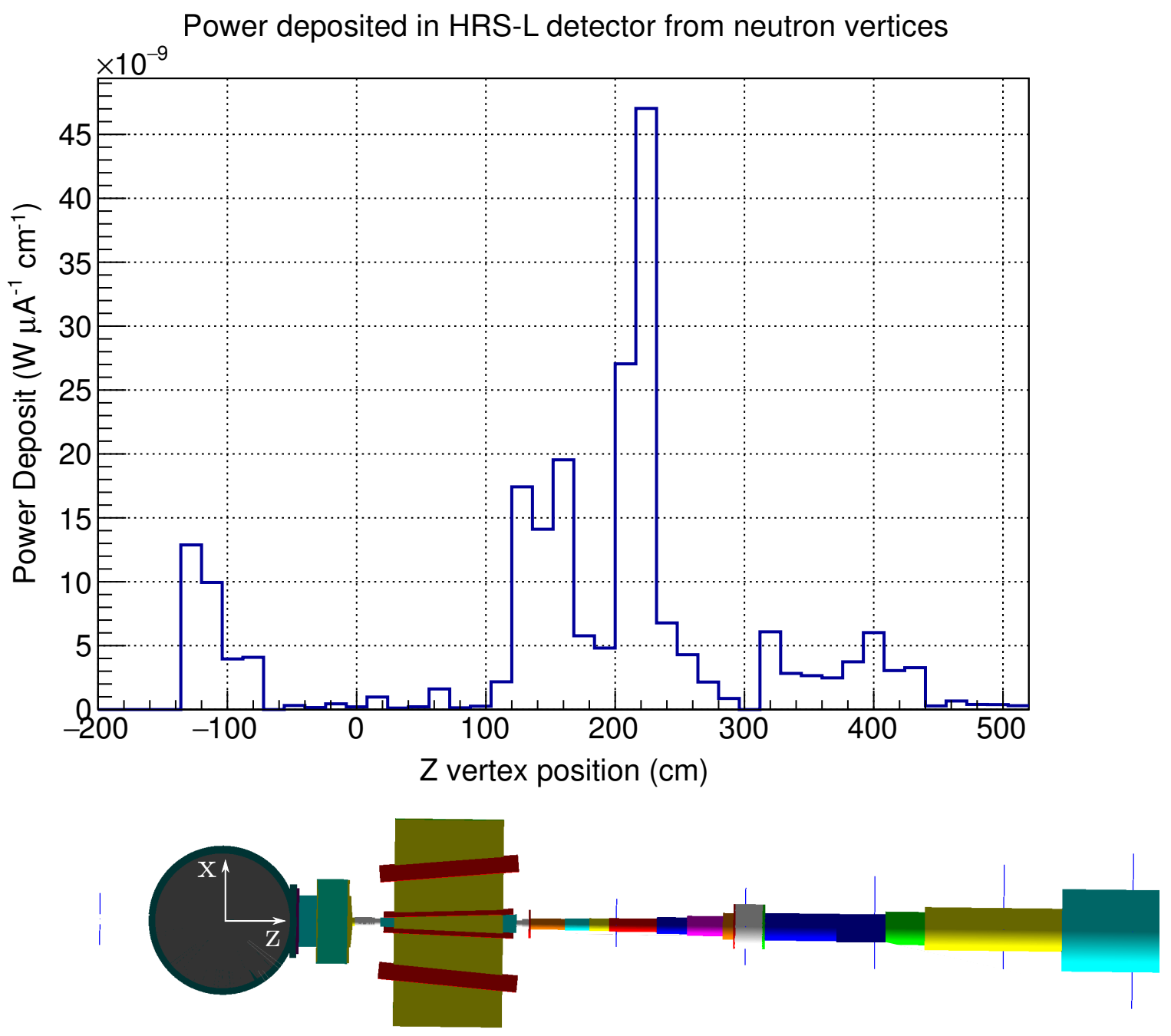

Figure 3.41: Simulated power deposition in the HRS-L volume due to neutrons for the APEX $3.3 \mathrm{GeV}$ experiment. Y-axis indicates the deposited power normalized by the bin size of the histogram and the $\mathrm{x}$-axis indicates the $\mathrm{z}$ coordinate of the primary vertices. Events in the histogram were limited to the vertices with an $\mathrm{x}$ coordinate in the range $-100 \mathrm{~cm}<\mathrm{x}<100 \mathrm{~cm}$. This is a blown-up view of the plot in Figure 3.39 . 
Telescopic section of the beam

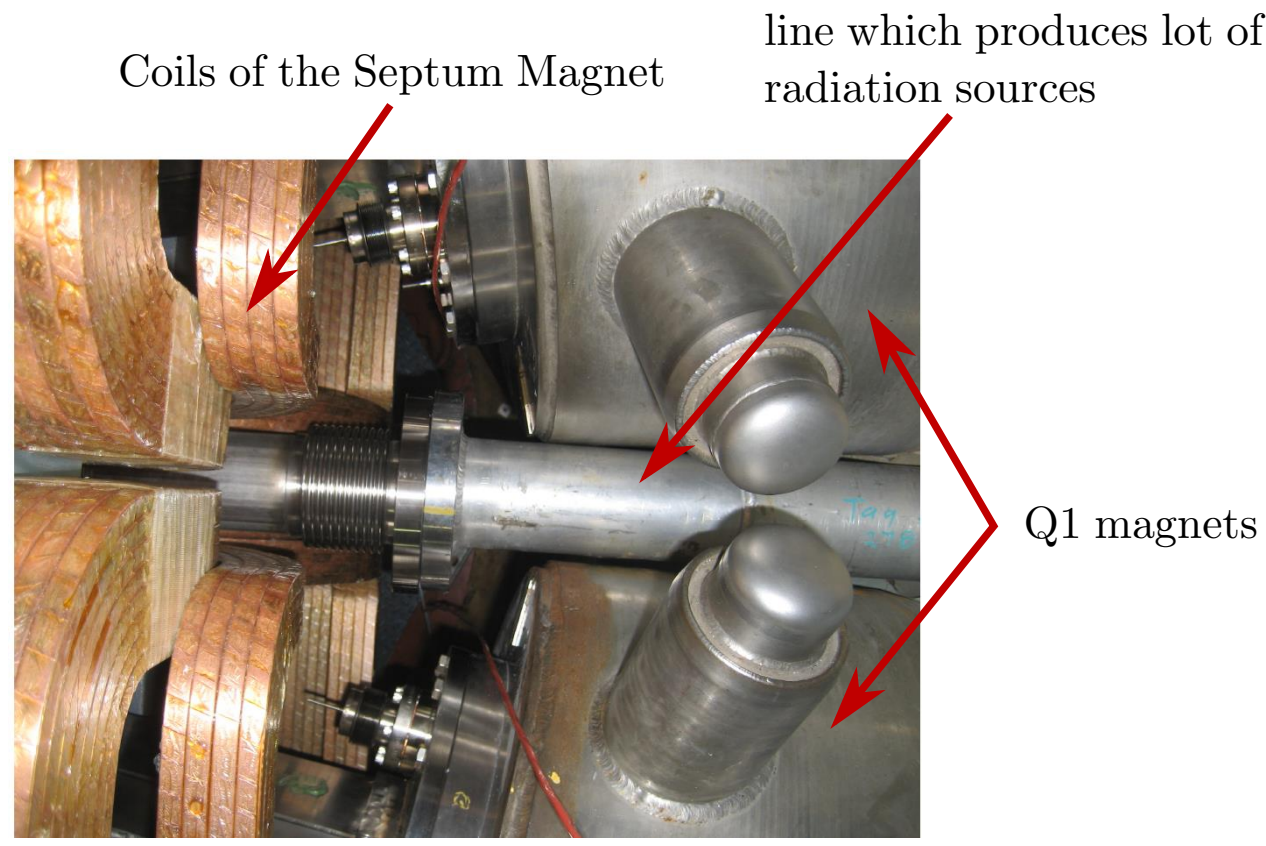

B. Wojtsekhowski

Figure 3.42: The photograph shows a top view of the setup around the telescoping beam pipe. Simulations identified that the most of the radiation reached the HRS-L scoring volume was originated in this section of the beam line. 
ers in the $\mathrm{x}^{\prime}$ direction, forming smaller segments that each have the dimensions of $20 \mathrm{~cm} \times 200 \mathrm{~cm} \times 5 \mathrm{~cm}$. The directions $\mathrm{x}^{\prime}, \mathrm{y}^{\prime}$ and $\mathrm{z}^{\prime}$ are defined with respect to the scoring volume. A small segment can be identified by its x'-layer and z'-layer number. The numbering scheme of these layers are shown in Figure 3.44. The layers corresponding to $\mathrm{x}^{\prime}=1$ and $\mathrm{z}^{\prime}=1$ respectively represent the faces of the scoring volume closest to the beamline. The volumes with higher $x^{\prime}$-layer and $z^{\prime}$-layer numbers are further away from the beamline.

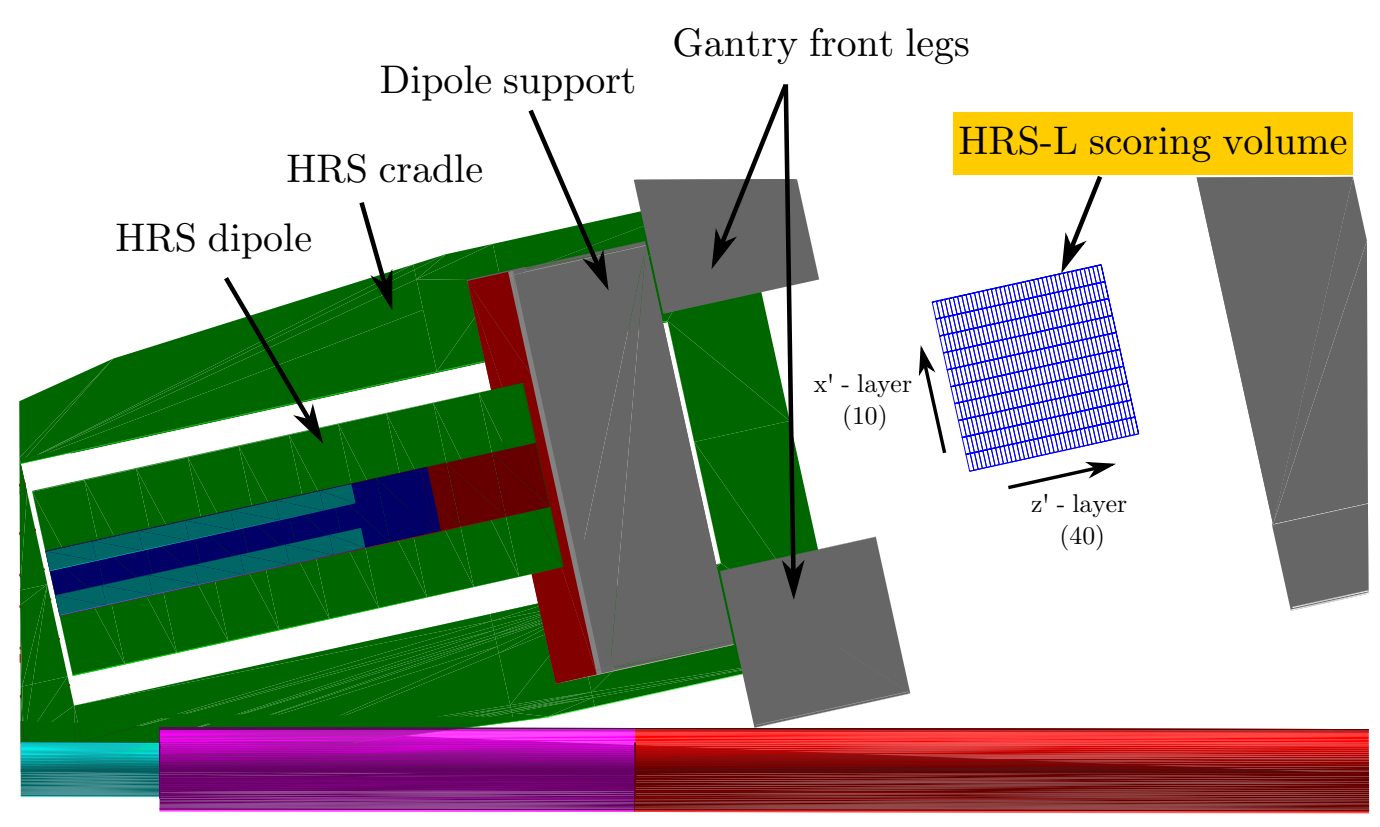

Figure 3.43: A top view of the left HRS. The beam line, components of the HRS and the HRS-L scoring volume can be seen in the image. The HRS-L scoring volume consists of 40 layers in the $\mathrm{z}^{\prime}$ direction and 10 layers in the $\mathrm{x}^{\prime}$ direction.

We calculated the energy deposition in the individual segments of the HRS-L scoring volume for the APEX $3.3 \mathrm{GeV}$ experiment. The energy deposition was converted to dose based on the particle type. Figure 3.45 illustrates dose distribution throughout the HRS-L scoring volume due to electrons. In this graph, the vertical axis indicates the dose in rem and the horizontal axis indicates the $\mathrm{x}^{\prime}$-layer number 


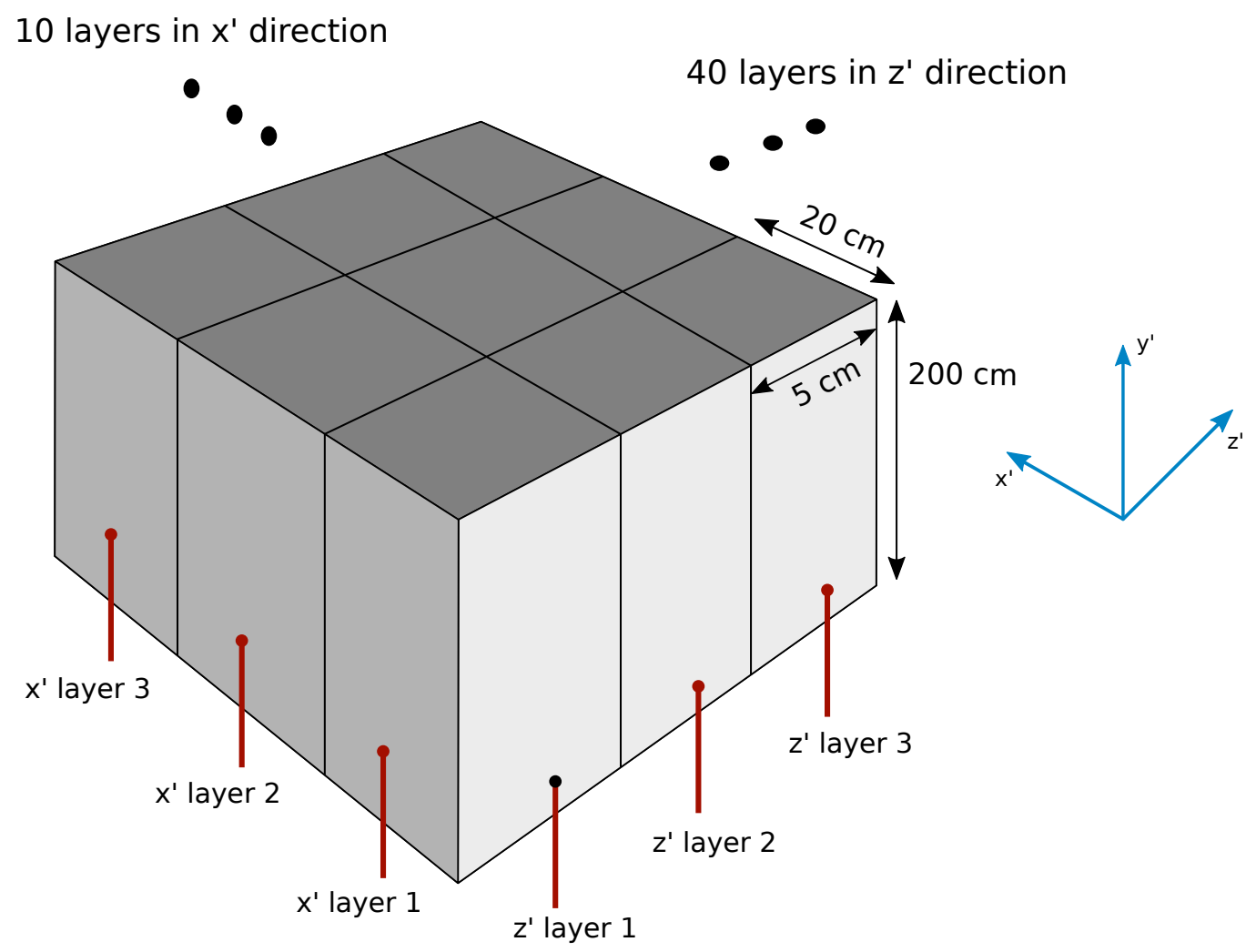

Figure 3.44: A $3 \mathrm{D}$ view of the HRS-L scoring volume. 
from 1 to 10. Figure 3.45 shows dose as a function of the $\mathrm{x}^{\prime}$ layer for several values of $\mathrm{z}^{\prime}$, and it can be seen that the layer corresponding to $z^{\prime}=1$ recieves significantly higher dose rates than layers for which $z^{\prime}>1$. Only the plots for the first $6 z^{\prime}$ layers are shown and only $z^{\prime} \leq 3$ are clearly visible. As can be seen in Figure 3.45, the dose drops by a factor of $\sim 2$ after passing through a $5 \mathrm{~cm}$ thick layer (first z layer). Figure 3.46 and Figure 3.47 illustrate the dose distribution in the HRS-L scoring volume due to photons and neutrons respectively. As can be seen in Figure 3.47, it takes about $15 \mathrm{~cm}$ thick polyethylene (3 layers in z direction) to reduce the dose by a factor of 2 . With this approach to shielding we would need a large area of polyethylene to cover the HRS electronics and also a fairly thick layer. Satisfying both of these criteria would probably be difficult. Therefore, we need a better approach to shield the HRS electronics from neutrons.

In the previous section we demonstrated that most of the radiation will be produced from a section of the beam line referred to as the telescoping beam pipe. The amount of material (polyethylene) needed to cover the telescoping beam pipe is smaller compared to that for the HRS electronics area. As can be seen in Figure 3.42, this section of the beam line has very limited space around it to add any shielding. Therefore, we studied an alternative method to lower the radiation at the HRS electronics. The idea was to collimate the electron beam before it enters the telescoping beam pipe and reduce the interactions with the beam line. We used Geant4 simulations to test this idea. A tungsten collimator was placed inside the beam pipe that goes through the septum magnet (septum beam pipe). We believe that the radiation produced in the collimator can get absorbed by the thick iron structure of the septum magnet, making this location ideal to install the collimator. Figure 3.48 shows the tungsten collimator, that was used in the Geant 4 simulations, which is about $10 \mathrm{~cm}$ in length and has a wall thickness of $1.5 \mathrm{~cm}$. The collimator has an inner diameter of 


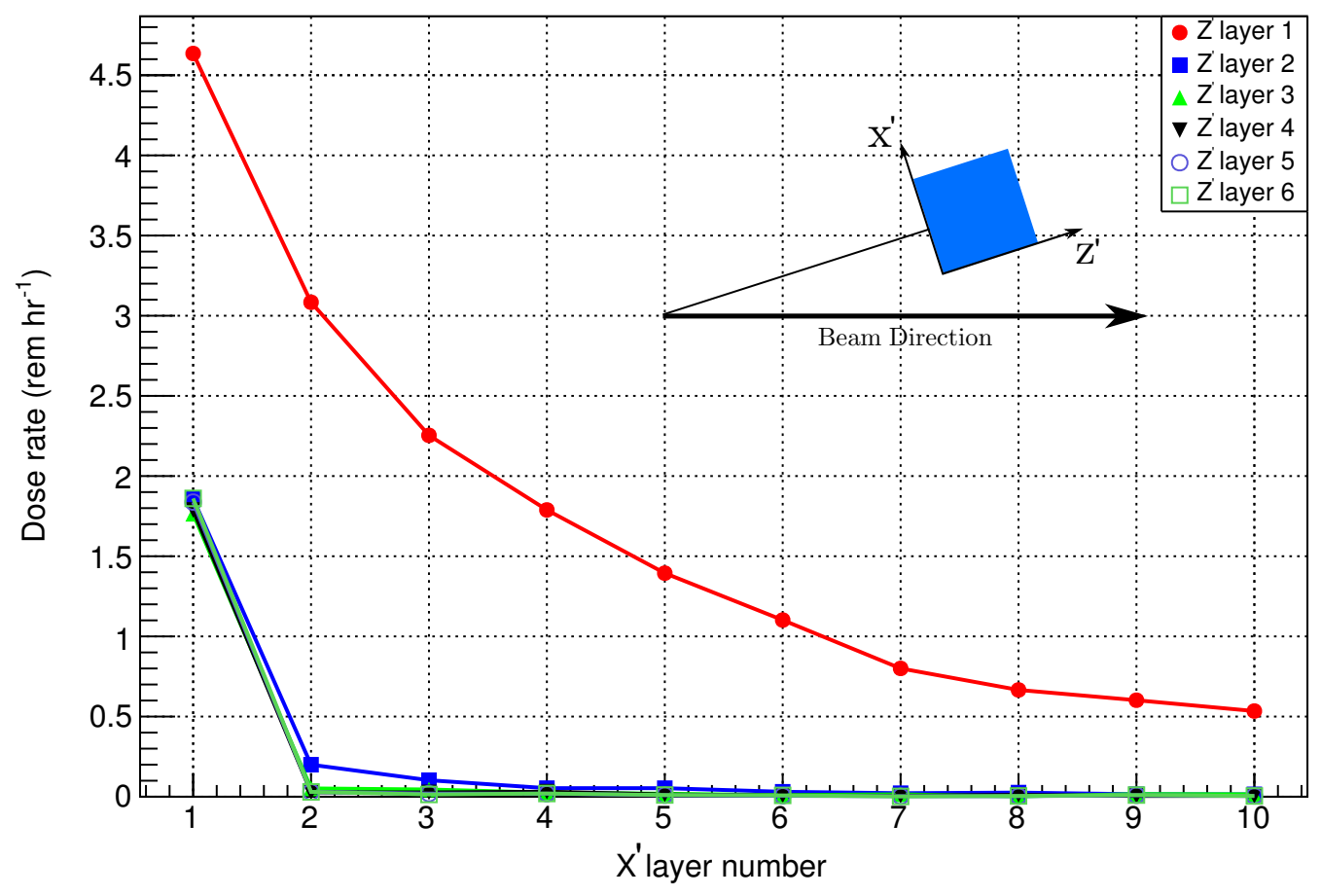

Figure 3.45: Electron dose distribution within the HRS-L scoring volume. Dose in each $z^{\prime}$-layer is plotted as a function of $\mathrm{x}^{\prime}$-layer number. Only $\mathrm{z}^{\prime}$-layers with significant dose difference are shown here. 


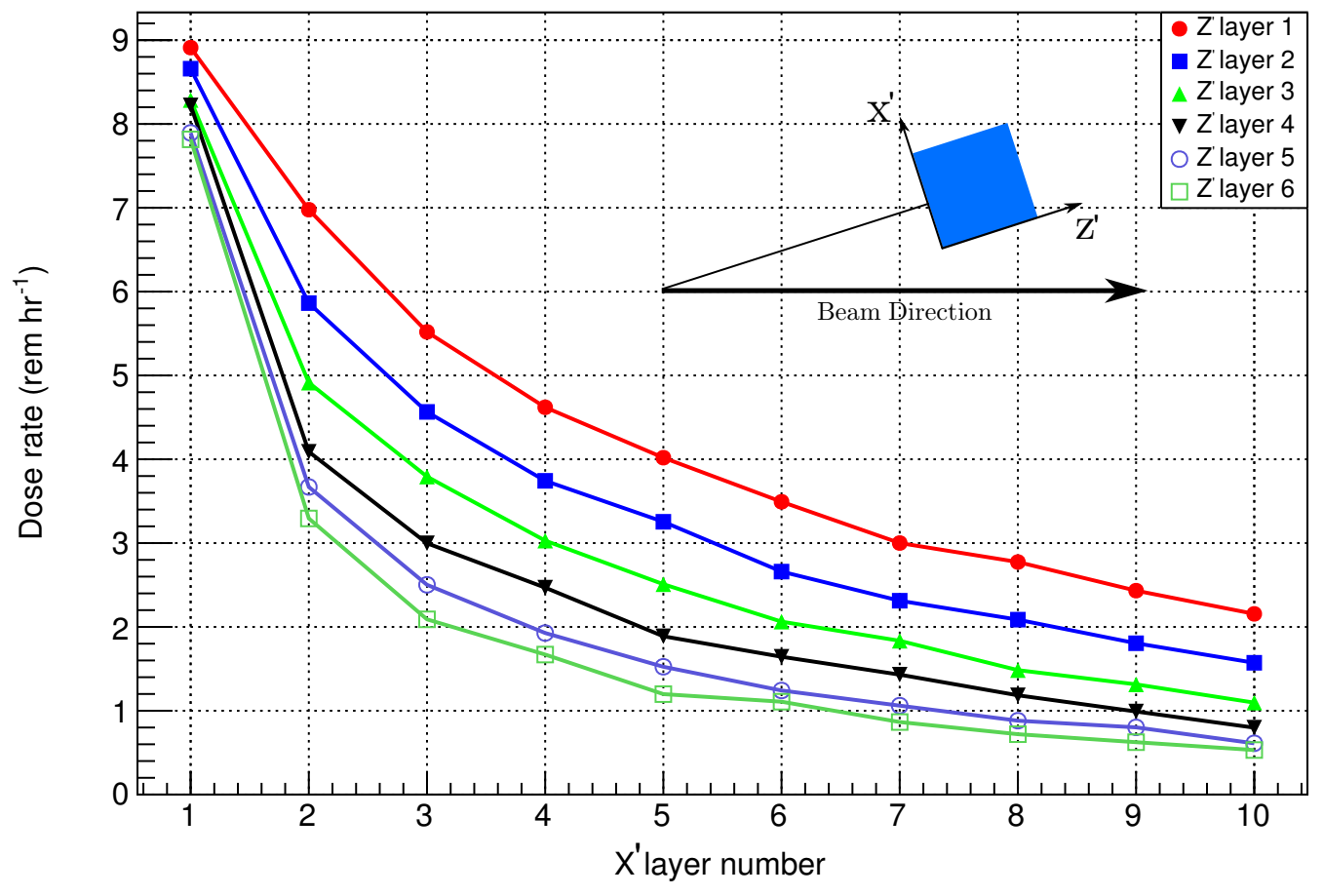

Figure 3.46: Photon dose distribution within the HRS-L scoring volume. Dose in each $\mathrm{z}^{\prime}$-layer is plotted as a function of $\mathrm{x}^{\prime}$-layer number. Only $\mathrm{z}^{\prime}$-layers with significant dose difference are shown here. 


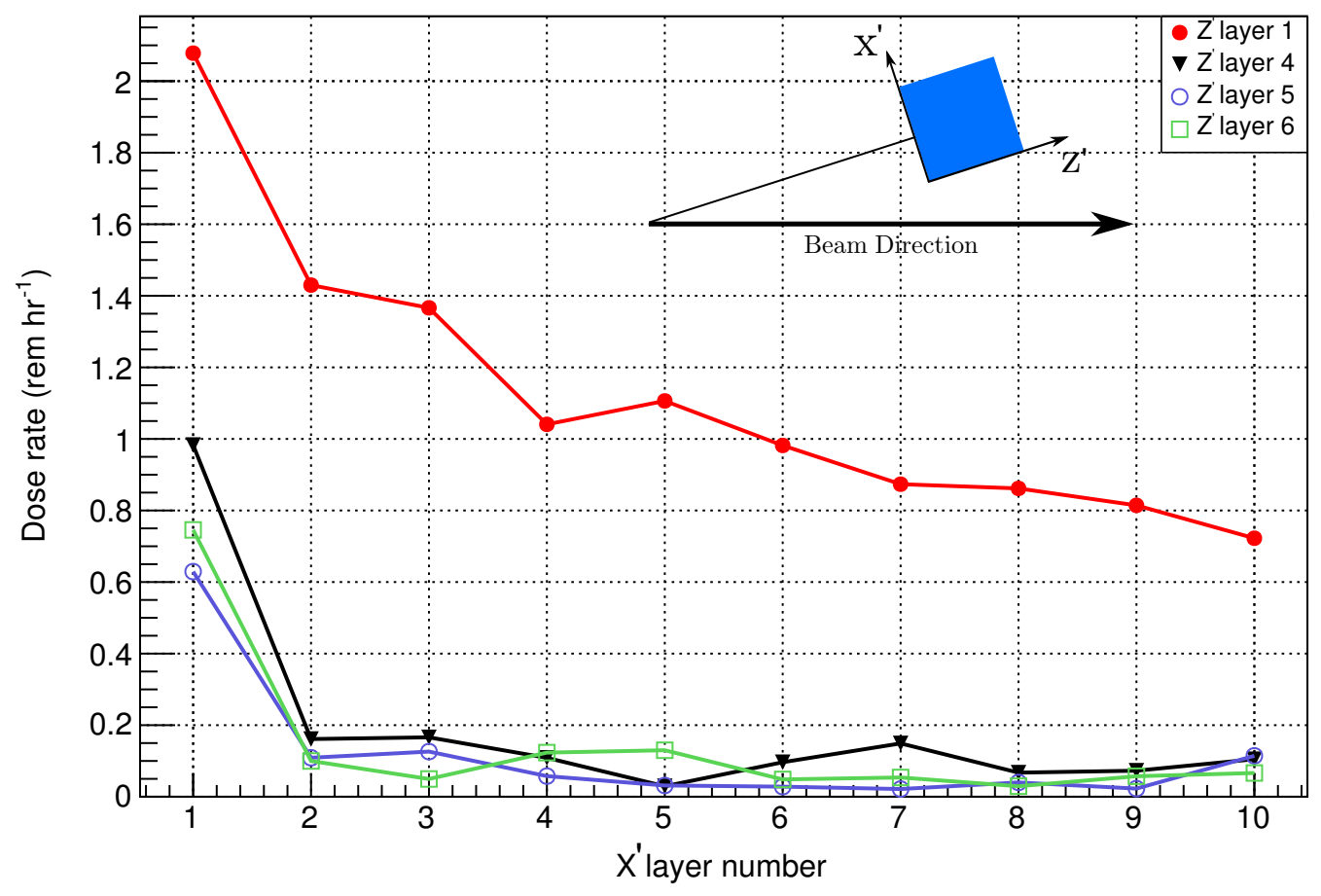

Figure 3.47: Neutron dose distribution within the HRS-L scoring volume. Dose in each $\mathrm{z}^{\prime}$-layer is plotted as a function of $\mathrm{x}^{\prime}$-layer number. Only $\mathrm{z}^{\prime}$-layers with significant dose difference are shown here. 
$3.2 \mathrm{~cm}$ which allows the electrons to pass. As illustrated in Figure 3.49, the collimator is located about $20 \mathrm{~cm}$ from the upper edge of the septum magnet.
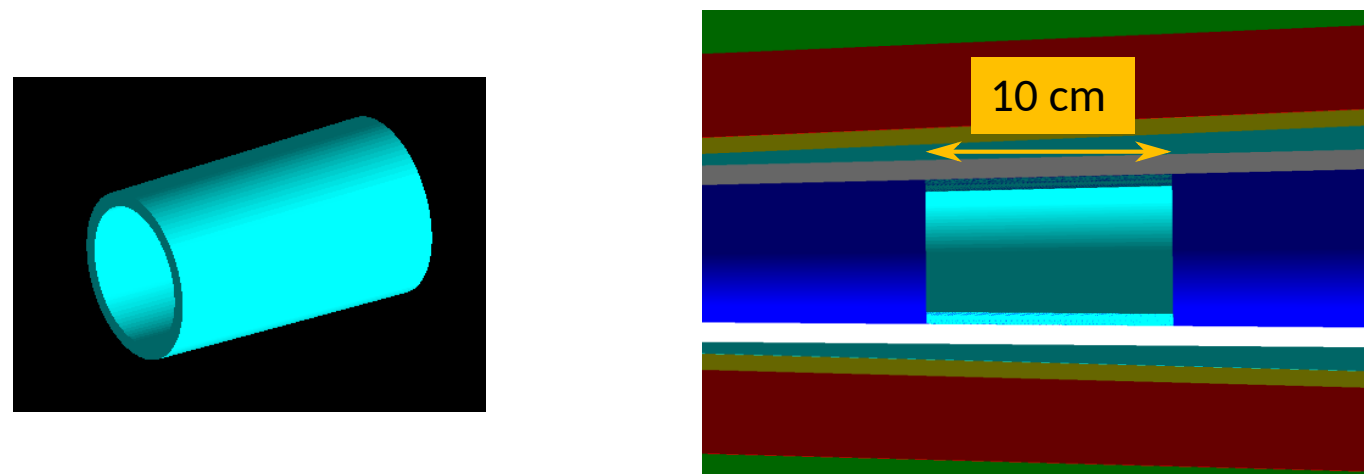

Figure 3.48: Tungsten collimator that is used to focus the beam towards the narrow section of the beam line (left). This collimator will be placed inside the magnetic beam pipe (right).

We simulated both APEX at $3.3 \mathrm{GeV}$ and $120 \mu \mathrm{A}$ and APEX at $4.4 \mathrm{GeV}$ and $90 \mu \mathrm{A}$ and calculated the damage to semiconductor electronics. We studied the change in the neutron spectra in the JLab Hall A with the addition of the tungsten collimator described above. Figure 3.50 illustrates two neutron energy spectra at the left HRS electronics, with and without the tungsten collimator for the APEX $3.3 \mathrm{GeV}$ experiment. The solid (black) line indicates the neutron energy spectrum without the collimator and the dashed (red) line indicates that with the collimator. The histograms have equal-width bins in the log scale and the fluence is per energy bin which is indicated by $\Phi(\mathrm{E}) \mathrm{dE}$. As can be seen in Figure 3.50, using a collimator has reduced the neutron fluence at the HRS electronics, especially neutrons with energies above $0.1 \mathrm{MeV}$. Even though the collimator helped to lower the neutron fluence at HRS electronics, it could increase the neutron fluence in other areas of the Hall A. Figure 3.51 illustrates a similar comparison of spectra in the upstream region of Hall A, where the tungsten collimator actually increased the neutron fluence. Based 


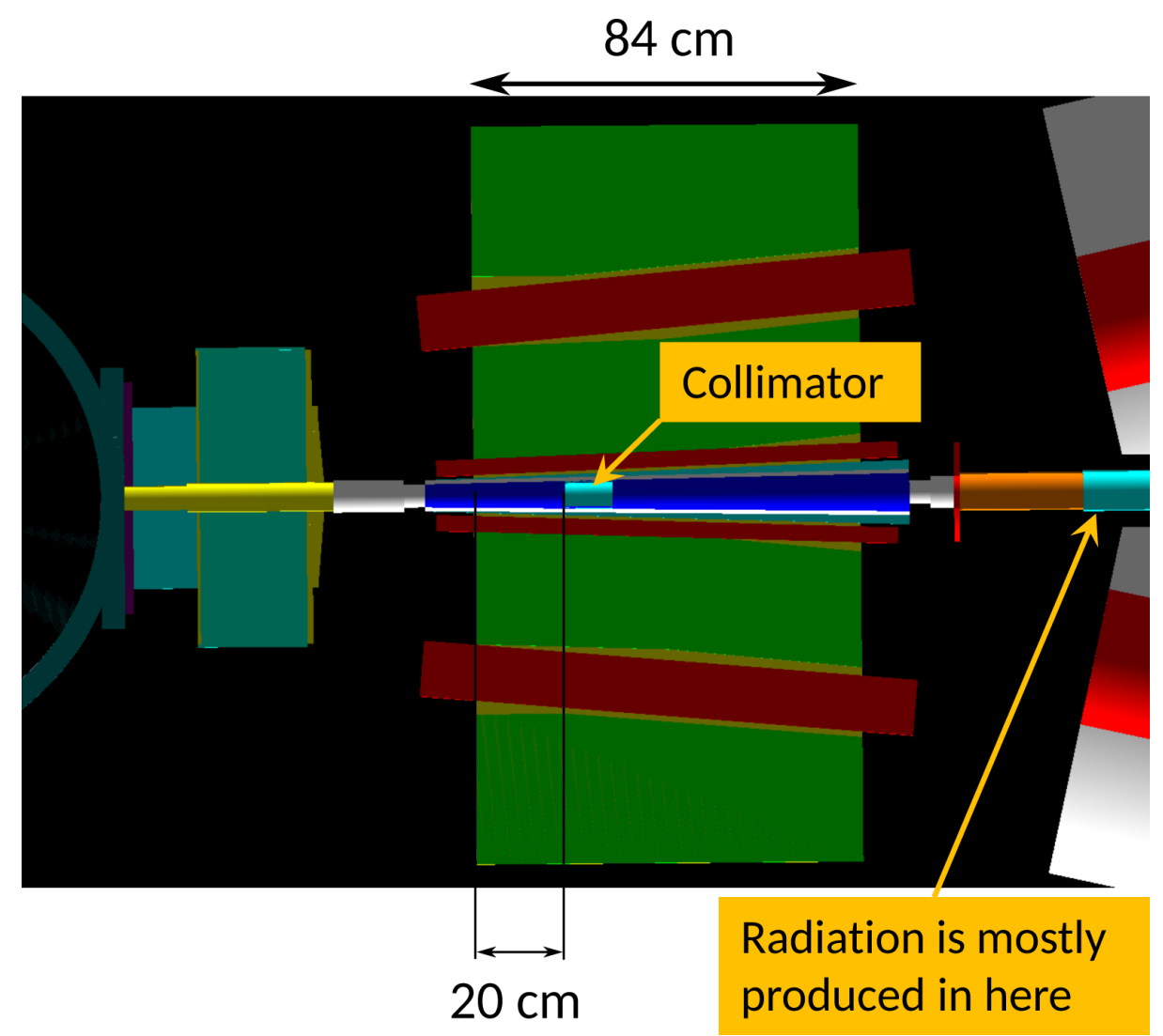

Figure 3.49: A horizontal cross-section of the septum magnet as seen from the top. The tungsten collimator is visible and it is located about $20 \mathrm{~cm}$ from the upper edge of the septum magnet. 
on the neutron spectra, we calculated the electronics damage in terms of the $1 \mathrm{MeV}$ equivalent neutron fluence. Table 3.6 summarizes the calculated $1 \mathrm{MeV}$ equivalent neutron fluence with and without the Tungsten collimator. Using the Tungsten collimator with a $1.5 \mathrm{~cm}$ thick wall in the septum beam pipe we were able to lower the neutron fluence at the HRS electronics area by a factor $\sim 3$. This resulted in an increase in the neutron fluence in the upstream region of the Hall A by a factor of $\sim 2$. Even though there is a factor of $\sim 2$ increase in the $1 \mathrm{MeV}$ equivalent fluence when the collimator is in use, it did not exceed the ESAD threshold value for electronic damage. Furthermore, there are no sensitive semiconductor electronics located in the upstream region of the Hall A. Further studies need to be done before the relative merits of this approach can be evaluated.

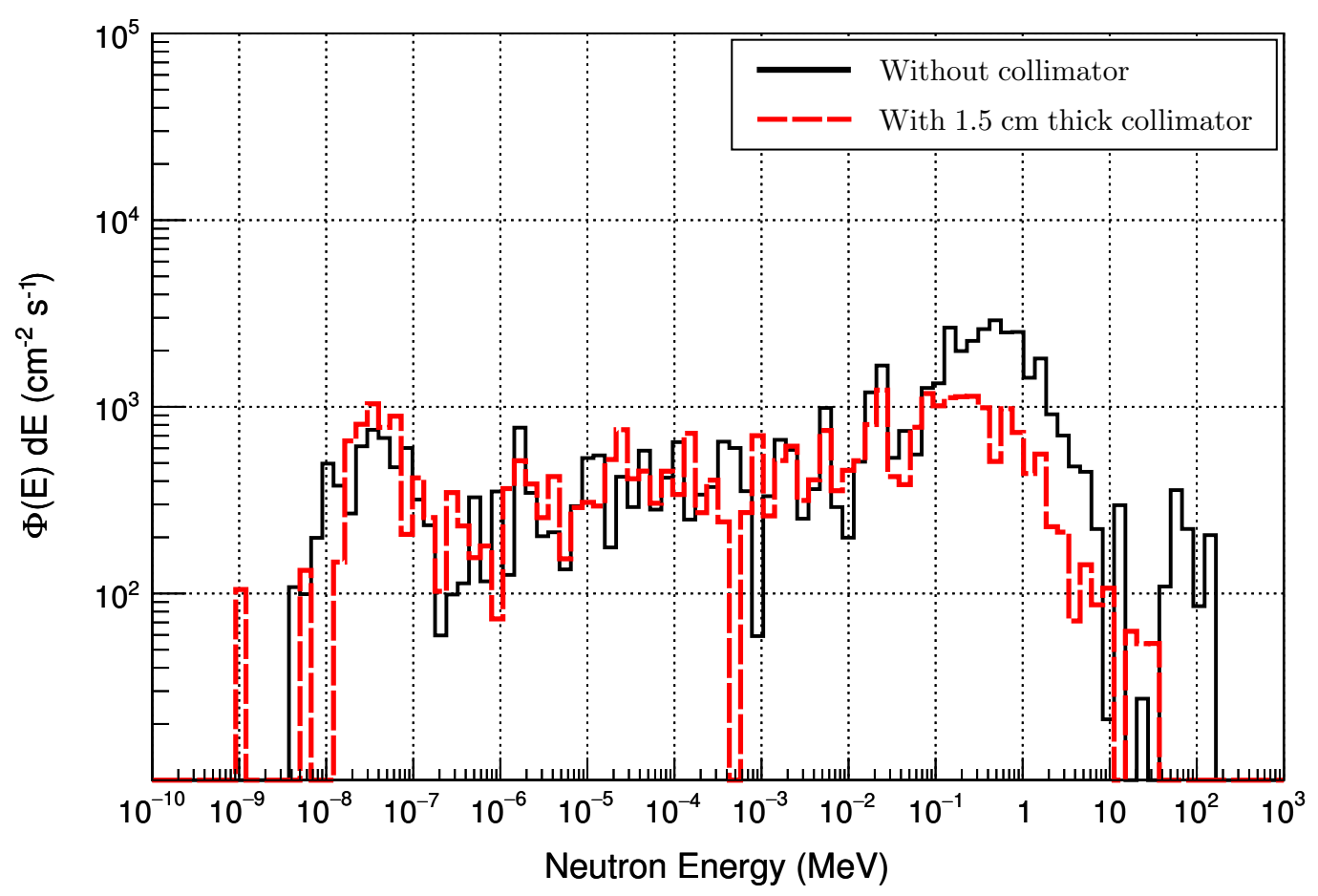

Figure 3.50: APEX $3.3 \mathrm{GeV}$ at $120 \mu \mathrm{A}$ beam current was simulated with and without the Tungsten collimator. Energy spectra of neutrons at HRS-L electronics area were compared. There is a reduction of neutrons above $0.1 \mathrm{MeV}$ when the collimator is being used. 


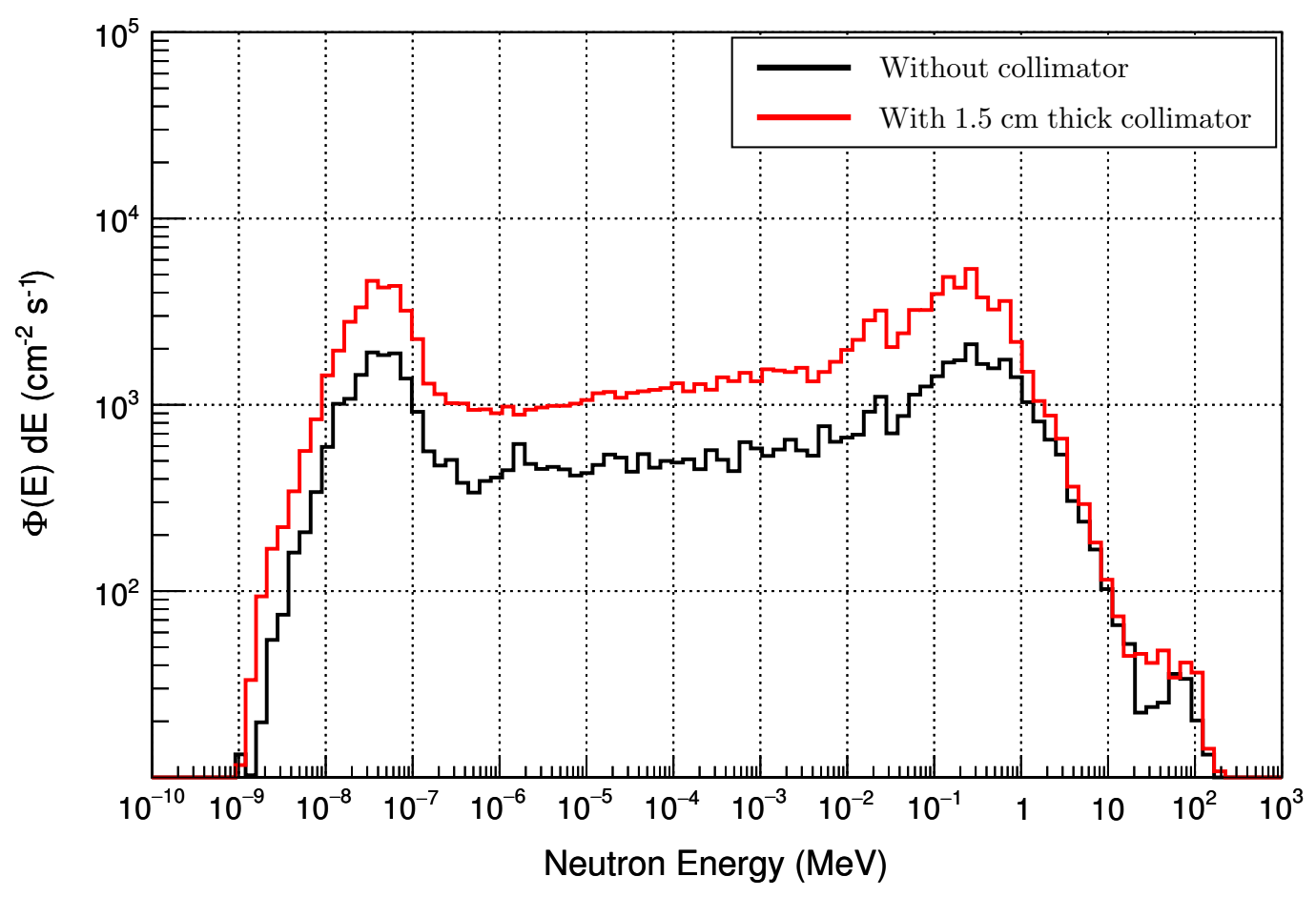

Figure 3.51: APEX $3.3 \mathrm{GeV}$ at $120 \mu \mathrm{A}$ beam current was simulated with and without the Tungsten collimator. Energy spectra of neutrons in upstream were compared. There is a increase of neutrons when the collimator is being used.

\subsection{A summary of Monte-Carlo simulation of ra- diation during APEX}

In this chapter, we focused on calculating radiation levels in JLab Hall A during the APEX experiment and compared them to past experiments. We started by developing a Geant4 model of Hall A and comparing several simulated results using our model with those done by others. This included a comparison with Geant3 simulations developed by Pavel Degtiarenko in the RadCon group and Geant4 simulations developed by Rakitha Beminiwattha for the PREX collaboration; we have very good agreement (see section 3.1). We also compared our simulations with several mea- 
Table 3.6: Calculated equivalent $1 \mathrm{MeV}$ neutron fluence at the HRS electronics area and in the upstream of the JLab Hall A. Only the APEX $3.3 \mathrm{GeV}$ and the APEX $4.4 \mathrm{GeV}$ settings were considered. The column "With Collimator" indicates the neutron fluence when a tungsten collimator with a wall thickness of $1.5 \mathrm{~cm}$ was used within the septum magnet.

\begin{tabular}{|c|c|c|c|c|c|}
\hline & & \multicolumn{4}{|c|}{$1 \mathrm{MeV}$ neutron fluence $\left(\mathrm{n}_{\mathrm{eq}} \mathrm{cm}^{-2}\right)$} \\
\hline & & \multicolumn{2}{|c|}{ HRS-L electronics area } & \multicolumn{2}{|c|}{ Upstream } \\
\hline & & $\begin{array}{c}\text { Without } \\
\text { Collimator }\end{array}$ & $\begin{array}{c}\text { With } \\
\text { Collimator }\end{array}$ & $\begin{array}{l}\text { Without } \\
\text { Collimator }\end{array}$ & $\begin{array}{c}\text { With } \\
\text { Collimator }\end{array}$ \\
\hline \multirow{2}{*}{ APEX $3.3 \mathrm{GeV}$} & $e^{+} / e^{-}$ & $3.1 \mathrm{E}+09$ & $8.1 \mathrm{E}+08$ & $6.2 \mathrm{E}+07$ & $6.5 \mathrm{E}+07$ \\
\hline & $\mathrm{n}$ & $1.1 \mathrm{E}+10$ & $3.4 \mathrm{E}+09$ & $6.6 \mathrm{E}+09$ & $1.2 \mathrm{E}+10$ \\
\hline \multirow{2}{*}{ APEX $4.4 \mathrm{GeV}$} & $e^{+} / e^{-}$ & $3.3 \mathrm{E}+09$ & $9.9 \mathrm{E}+08$ & $8.5 \mathrm{E}+07$ & $8.4 \mathrm{E}+07$ \\
\hline & $\mathrm{n}$ & $1.3 \mathrm{E}+10$ & $4.4 \mathrm{E}+09$ & $8.4 \mathrm{E}+09$ & $1.4 \mathrm{E}+10$ \\
\hline
\end{tabular}


surements. When we compared our calculations for PREX-I with a one type of measurement, based on the dark current induced in a Silicon Photomultiplier, we got reasonably close agreement (see section 3.4.1). But, when we compared with another type of measurement, using a SNOOPY during PREX-I and RCS, we have large discrepancies. To compare a calculated fluence with a radiation detector, we need to understand several issues, including the response function of the detector (in this case a SNOOPY) that was used for the measurement and also the possibility of dead-time. We simulated the response function of a SNOOPY and we have excellent agreement with other authors (see section 3.4.2). When we take the correct response function into account, and when we also make corrections for dead-time using as guidance the work of Moyers et al., our simulations appear to be in reasonable agreement with what we predict the SNOOPY measurements should have seen.

In summary, we believe that our simulations of the radiation to be expected during the APEX experiment are reliable, having stood up to multiple tests. Our simulations have yielded results extremely close to simulations conducted by others. Furthermore, when our simulations for PREX-I are compared with the measurements based on the dark current of SiPM's, we again see close agreement. When compared with measurements based on neutron radiation detectors (SNOOPY's), we again see reasonable agreement, but only if the effects of the response curves and dead time are taken into effect. While we readily admit that the issue of dead time requires more study, we note that this effect only appears to be large in the case of PREX-I, an experiment that may well have produced higher radiation levels than any previous experiment at JLab. It is not surprising that PREX-I should be the first experiment in which the dead time of neutron radiation detectors became an issue.

On the question of estimating the errors of our simulations, we are faced with many of the same issues that face anyone working with programs such as GEANT 
and FLUKA. One often hears comments along the lines of: "anything within a factor of two is reasonable". Indeed, when all corrections (some better understood than others) are taken into account, the worst discrepancies we see with measured values are on that order, a factor of two. In the case of the comparison with the SiPM tests, an approach that is considerably cleaner in certain ways, our agreement is much closer. Our central conclusion, that APEX will have radiation levels around an order of magnitude lower than those during PREX-I, appears quite robust. If it is determined that a factor of two error in our prediction is worrisome, one could always pursue some of the radiation-reducing strategies outlined in section 3.5. 


\section{Chapter 4}

\section{Scintillating Fiber Detector (SciFi)}

Because APEX will detect positrons in one arm of the High Resolution Spectrometer (HRS), some of the usual techniques for understanding the optics of the spectrometer will not work. For this reason, an alternative strategy has been developed that relies on a novel Scintillating Fiber (SciFi) detector.

\subsection{Mass resolution}

The $A^{\prime}$ will appear as a small peak on top of a background of $e^{+} e^{-}$pairs resulting from radiative pair production and Bethe-Heitler process (QED trident backgrounds). The width of the peak corresponds to the mass resolution of the experiment. Therefore, precise calculation of mass resolution is very important.

Calculation of invariant mass of $e^{+} e^{-}$pair and the mass resolution is shown below. Considering the decay of the $A^{\prime}$ to an $e^{+} e^{-}$pair, the invariant mass can be written as,

$$
m_{0}^{2}=\left(E_{e^{+}}+E_{e^{-}}\right)^{2}-\left(\vec{p}_{e^{+}}+\vec{p}_{e^{-}}\right)^{2}
$$


Expanding we have:

$$
m_{0}^{2}=E_{e^{+}}^{2}+E_{e^{-}}^{2}+2 \cdot E_{e^{+}} \cdot E_{e^{-}}-\vec{p}_{e^{+}}^{2}-\vec{p}_{e^{-}}^{2}-2 \cdot \vec{p}_{e^{+}} \cdot \vec{p}_{e^{-}}
$$

and substituting $m_{e^{ \pm}}^{2}=E_{e^{ \pm}}^{2}-\vec{p}_{e^{ \pm}}^{2}$ gives

$$
m_{0}^{2}=m_{e^{+}}^{2}+m_{e^{-}}^{2}+2 \cdot E_{e^{+}} \cdot E_{e^{-}}-2 \cdot \vec{p}_{e^{+}} \cdot \vec{p}_{e^{-}}
$$

Since the masses of $e^{+}$and $e^{-}$are negligible, it is approximately the case that $E_{e^{ \pm}}=$ $p_{e^{ \pm}}$, and we can write

$$
m_{0}^{2}=2 \cdot\left|\vec{p}_{e^{+}}\right|\left|\vec{p}_{e^{-}}\right|-2 \cdot \vec{p}_{e^{+}} \cdot \vec{p}_{e^{-}}
$$

If, $\theta$ is the angle between an $e^{+} e^{-}$pair, then

$$
m_{0}^{2}=2 \cdot\left|\vec{p}_{e^{+}}\right|\left|\vec{p}_{e^{-}}\right|(1-\cos \theta)
$$

So

$$
m_{0}^{2}=4 \cdot\left|\vec{p}_{e^{+}}\right|\left|\vec{p}_{e^{-}}\right| \sin ^{2} \frac{\theta}{2}
$$

Since the angle $\theta$ is small,

$$
m_{0} \approx\left|\vec{p}_{e^{+}}\right|^{1 / 2}\left|\vec{p}_{e^{-}}\right|^{1 / 2} \cdot \theta
$$

and the mass resolution of the spectrometer, $\delta_{m}$, can be roughly given by,

$$
\left(\frac{\delta_{m_{0}}}{m_{0}}\right)^{2}=\frac{1}{2}\left(\frac{\delta_{p}}{p}\right)^{2}+\left(\frac{\delta_{\theta}}{\theta}\right)^{2}
$$


where $\delta_{\theta}$ is the angular resolution of the electron or positron, and $\delta_{p}$ is the momentum resolution of the HRS. Because of the excellent HRS relative momentum resolution of $3 \times 10^{-4}$, the mass resolution is dominated by three contributions to the angular resolution $\delta_{\theta}$ : scattering of the $e^{+} e^{-}$pairs inside the target, track measurement errors by the HRS detectors, and imperfections in the magnetic optics reconstruction matrix. For example, for the APEX test run where they used a $22 \mathrm{mg} / \mathrm{cm}^{2}$ thick Tantalum foil target, multiple scattering contributed $0.37 \mathrm{mrad}$ for both vertical and horizontal angular resolutions for each particle. Track measurement errors have contributed $0.33(1.85) \mathrm{mrad}$ to the horizontal (vertical) angular resolution in the left HRS and $0.43(1.77) \mathrm{mrad}$ in the right HRS. They have found uncertainties due to magnetic optics imperfections to be $0.10(0.22)$ mrad to the horizontal (vertical) angular resolution [31]. Multi-foil target that will be used in the APEX experiment will reduce the multiple scattering contribution to the angular resolution. For the ranges of coupling strength, $\alpha^{\prime}$, of the dark photon probed by this experiment (see section 1.2.2), the lifetime of the $A^{\prime}$ is long enough to decay outside the target foils. Therefore, there is no contribution from multiple scattering to the mass resolution of positron-electron pairs originated from an $A^{\prime}$.

The sieve slit method discussed in the next section is typically used to calibrate HRS optics. HRS angular resolution depends on how well you can perform the calibration. The typical values for HRS angular resolution is, $\sim 0.5 \mathrm{mrad}$ in the horizontal direction and $\sim 1 \mathrm{mrad}$ in the vertical direction.

\subsection{Optics calibration using sieve slit method}

The determination of position and angle of $e^{+} e^{-}$pairs at the interaction vertex is critical, when calculating the invariant mass. Particles leaving the target, travel 
through a series of magnets, three quadrupole magnets and a dipole magnet (QQDQ configuration), which we refer to here as the spectrometer optics. The focal plane coordinates of a detected particle are measured by the vertical drift chambers (VDCs). The spectrometer's optics matrix maps the focal plane coordinates and angle to the interaction vertex. These matrix elements need to be optimized using a calibration method to provide an accurate and precise measurement of the vertex of $e^{+} e^{-}$pairs. The sieve slit method has typically been the approach for the hall A HRSs.

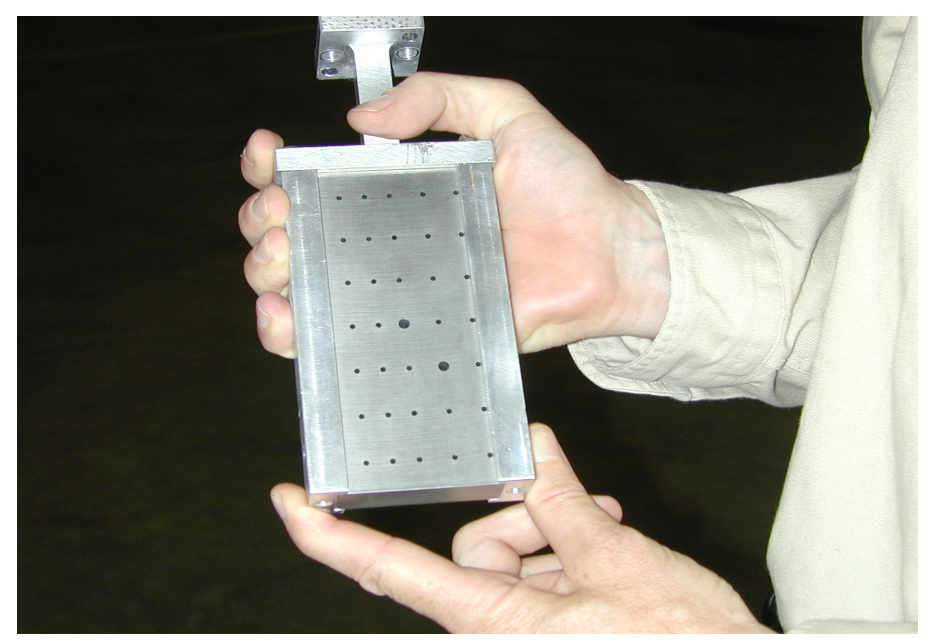

Figure 4.1: A model of a Tungsten sieve plate.

As illustrated in Figure 4.1, the sieve plate is a $5 \mathrm{~mm}$ thick Tungsten plate with a pattern of holes. The diameters of the holes are $1.4 \mathrm{~mm}$ and $2.8 \mathrm{~mm}$ and are drilled in a rectangular pattern. During a calibration run, the sieve plate is positioned between the target and the entrance to HRS collimator. Electrons and positrons lose energy when passing through the sieve plate, so that only particles that pass through holes reach the detector. Figure 4.2 shows the position of the sieve plate. Tracks of these detected events will be reconstructed back to the sieve plane and their positions at the sieve plane are compared with surveyed hole locations. These reconstructed trajectories of the electrons and positrons projected at the sieve plane should resemble 
the sieve hole pattern. By studying the deviation between surveyed and reconstructed hole positions and widths, one can optimize the coefficients in the matrix that is used to reconstruct particle tracks. Figure 4.3 taken from [71] shows an example of a sieve hole pattern.

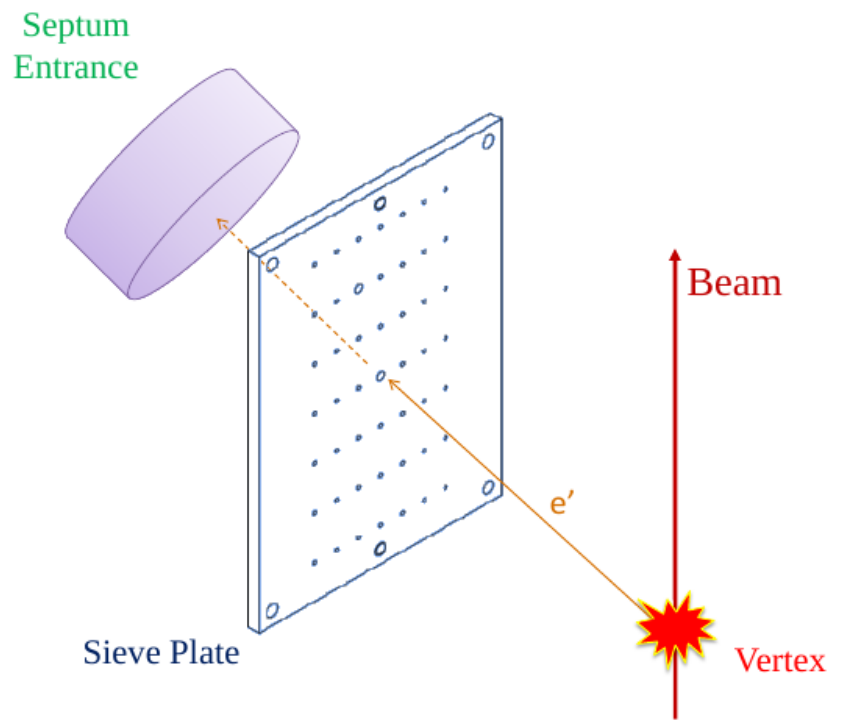

Figure 4.2: During a calibration run, the sieve plate is positioned between the target and the entrance to spectrometer. Analyzing the reconstructed and surveyed hole locations in the sieve plane, the reconstruction matrix can be optimized.

The errors of HRS track measurements and the final optics reconstruction matrix need to be calculated to evaluate angular resolution. The $\mathrm{x}$ and $\mathrm{y}$ distributions of each hole can be fit with a gaussian distribution. The reconstructed position and width of the hole can be extracted from the fit parameters. Figure 4.4 taken from [71] illustrates a gaussian fit to a $\mathrm{x}$ and $\mathrm{y}$ distribution of a sieve hole during APEX test run optics calibration. The track measurement uncertainty of the HRS detectors can be determined by comparing the reconstructed and surveyed widths of the sieve 

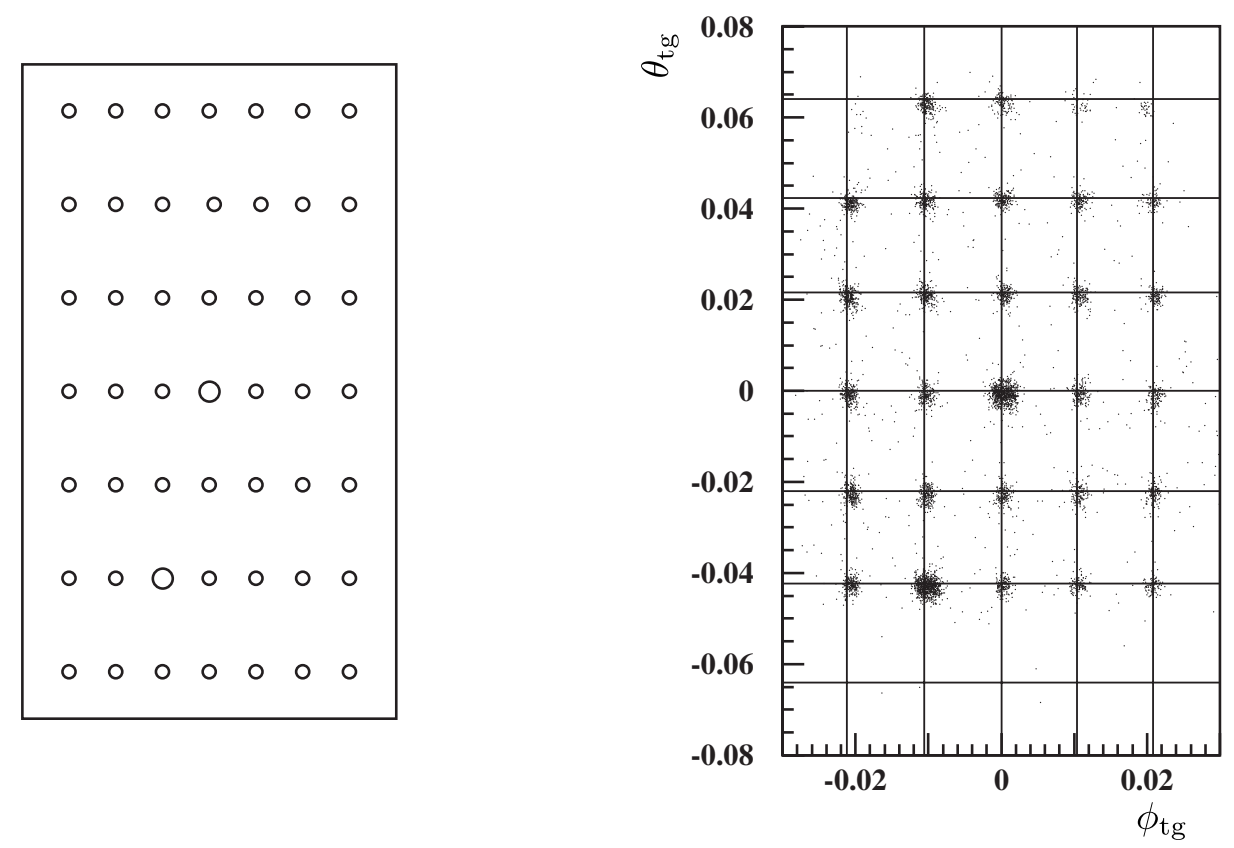

Figure 4.3: The geometric (left) and reconstructed (right) configurations of the sive slit. The large holes helps to identify the orientation of the image at the focal plane.

hole.

$$
\Delta_{\sigma_{x}}^{2}=\sigma_{x_{\text {reconstructed }}^{2}}^{2}-\sigma_{x_{\text {surveyed }}}^{2}, \Delta_{\sigma_{y}}^{2}=\sigma_{y_{\text {reconstructed }}}^{2}-\sigma_{y_{\text {surveyed }}^{2}}^{2}
$$

The uncertainty of the optics calibration matrix can be determined by comparing the reconstructed and surveyed hole positions.

$$
\Delta_{x}=x_{\text {reconstructed }}-x_{\text {surveyed }}, \Delta_{y}=y_{\text {reconstructed }}-y_{\text {surveyed }},
$$

These results can be converted to angular uncertainties by dividing by the distance to target (typically $\sim 1 \mathrm{~m}$ ) and taking inverse tangent. Contribution from track reconstruction errors and imperfections in the optics matrix averaged over all the sieve holes can be considered as the angular resolution of the HRSs. 

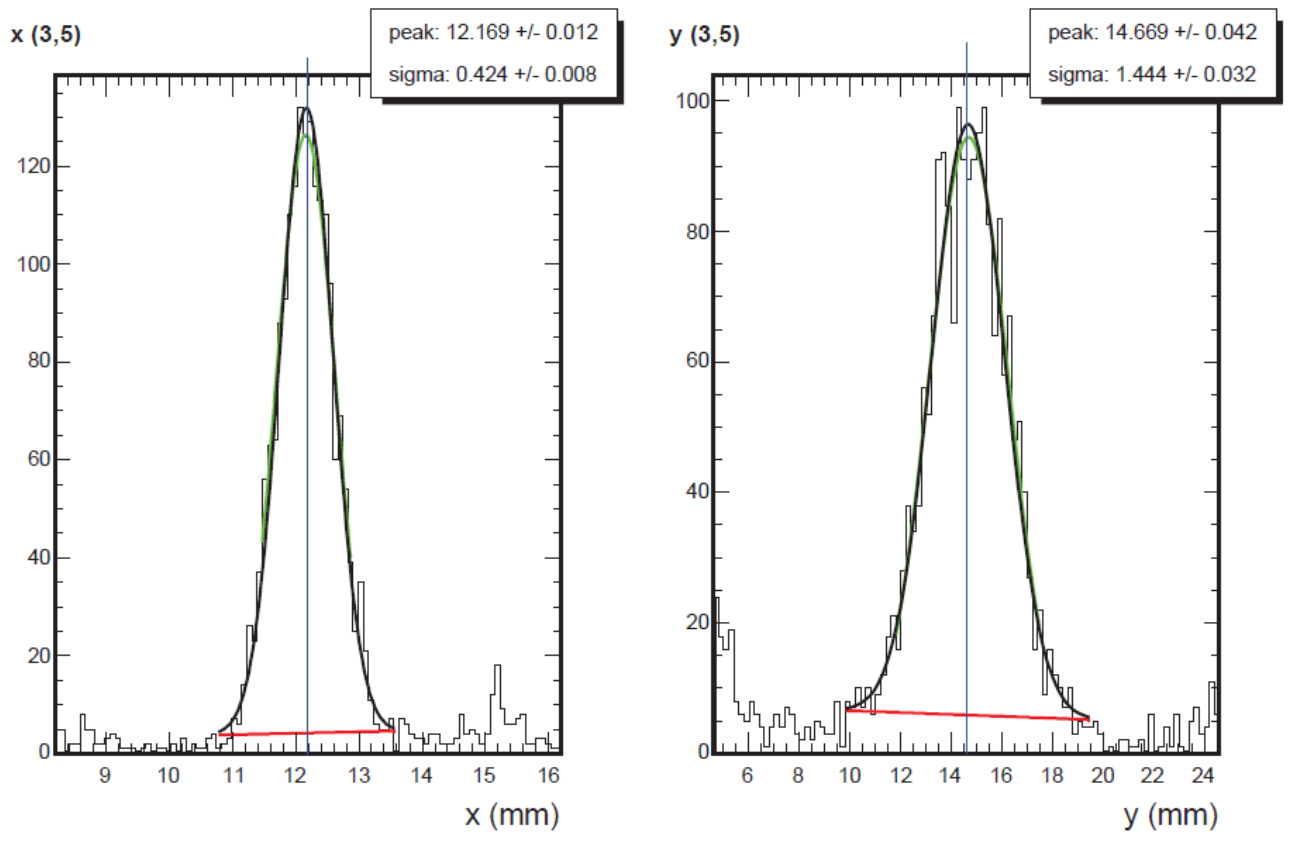

Figure 4.4: The $\mathrm{x}$ and $\mathrm{y}$ distributions for a single sieve hole. The distributions were fit with a guassian (black) on top of a linear background (red). The blue line indicates the surveyed location of the hole. The mean and the sigma of the fit correspond to the reconstructed position and width of the hole [71] [31]. 


\subsection{Optics calibration using an active scintillation fiber (SciFi) detector}

Even though the calibration with the sieve slit works for HRS with negative polarity, there are some practical problems working with positive polarity. In the APEX, the HRS optics will be calibrated to half of the beam energy. When electrons get elastically scattered form the target and hit on the sieve plate, these electrons produce positrons with momentum equal to that of the HRS momentum setting. These additional positrons would make the sieve holes impossible to distinguish. For the APEX test run this problem was resolved by calibrating both arms to negative polarity and using the results to infer the calibration coefficients for positive polarity of the arm. This process needs a large amount of time to flip the polarity of the HRS and might introduce additional uncertainty to the track reconstruction in the positive arm. This would lower the APEX invariant mass resolution. Simulation studies show that increasing the thickness of the sieve plate will not help to resolve this problem in the positive arm [72]. For these reasons a new method of calibration using an active detector has been pursued instead of further optimizing the sieve slit method. This method will be used to calibrate both arms in the HRS during APEX experiment.

\subsection{SciFi detector}

A novel Scintillating Fiber (SciFi) detector was constructed to perform a better optics calibration in the both arms of the HRS [73]. The active area of the SciFi detector is $8.8 \mathrm{~cm} \times 10.3 \mathrm{~cm}$ and contains two planes of 32 round scintillating fibers of $1 \mathrm{~mm}$ diameter. Fibers in one plane are oriented vertically with a center-to-center spacing of $2.79 \mathrm{~mm}$. Fibers in the other plane are oriented horizontally with a spacing of 
$3.3 \mathrm{~mm}$. The two planes of fibers are parallel to each other. Figure 4.5 illustrates the active area of the SciFi detector. The fibers are glued to an aluminum frame. To avoid fiber buckling at temperatures above room temperature due to differences in thermal expansion, an additional support is added to each layer of fibers by gluing the fibers to a 0.005 inch thick Kapton film using epoxy.

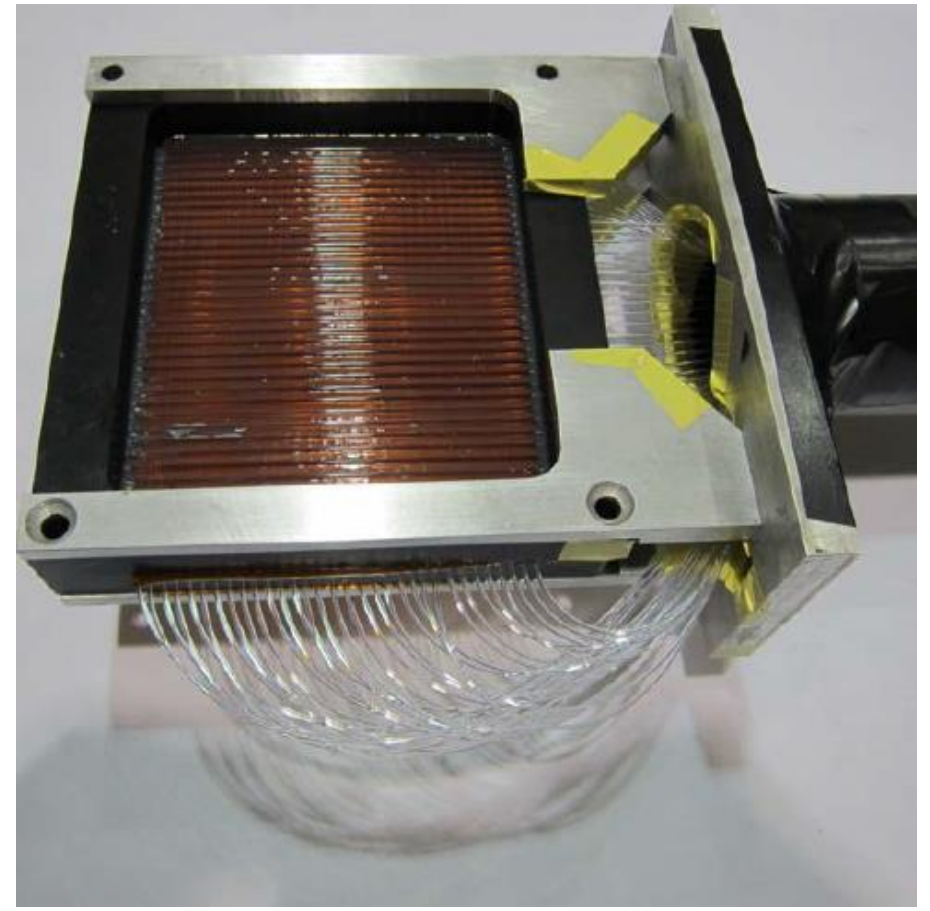

Figure 4.5: The active area of the Scintillating Fiber (SciFi) detector.

As is visible in Figure 4.6 taken from [73], the scintillating fibers are then sent through two guide brackets (labeled A and B) that sit along a horizontal arm. The guide brackets each have 64 holes for fibers to pass through and keep them aligned in a straight line. At the end of the horizontal arm, the fibers were threaded through a separator labeled as $\mathrm{C}$ in the same figure. Epoxy has been applied to fill the gaps between fibers and the separator. Fiber ends have been cut, polished and flushed with the face of the separator. Through another separator, the scintillating fibers are 
optically coupled to clear $1.5 \mathrm{~mm}$ diameter optical fibers that transport the scintillation light to a 64 channel multi-anode PMT (maPMT). This is necessary to locate the PMT setup away from the radiation and magnetic fields. As shown in the Figure 4.7, both separators connected to scintillating $1 \mathrm{~mm}$ fibers and clear $1.5 \mathrm{~mm}$ fibers have 64 holes arranged in a similar pattern. The separators attach together aligning each scintillating fiber with corresponding optical fiber. Each fiber from the SciFi connects to a slightly larger diameter optical fiber in order to reduce the loss of light at the boundary. The 64 clear $1.5 \mathrm{~mm}$ diameter optical fibers guide the scintillation light to a Hamamatsu 64 channel maPMT. To move the active area in and out of the beam, the detector is connected to a stepper motor. Figure 4.8 shows a complete setup of SciFi detector.

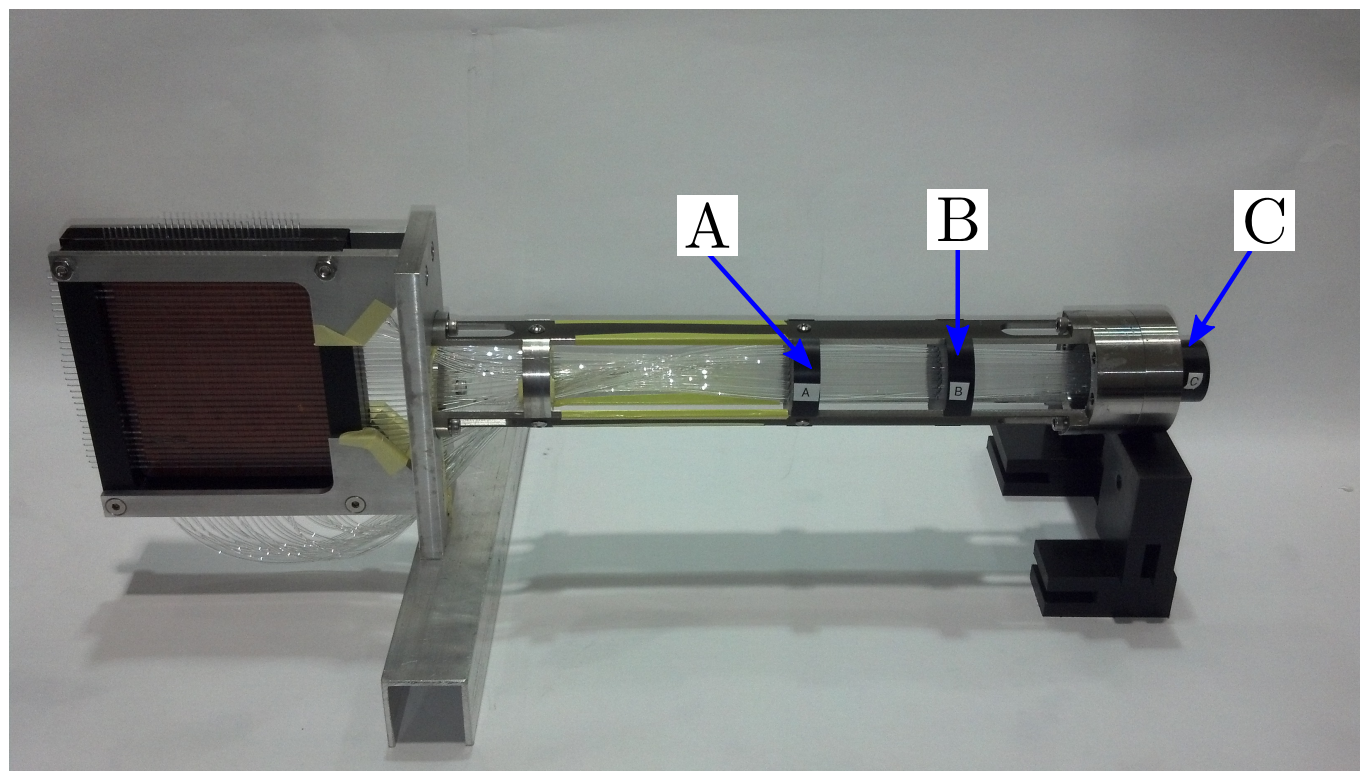

Figure 4.6: All 64 fiber ends are threaded through two guide brackets (A and B) and connected to a separator $(\mathrm{C})$.

During the APEX experiment two SciFi detectors will be used, one for each HRS. They will be positioned about $1 \mathrm{~m}$ downstream from the target inside the vacuum 


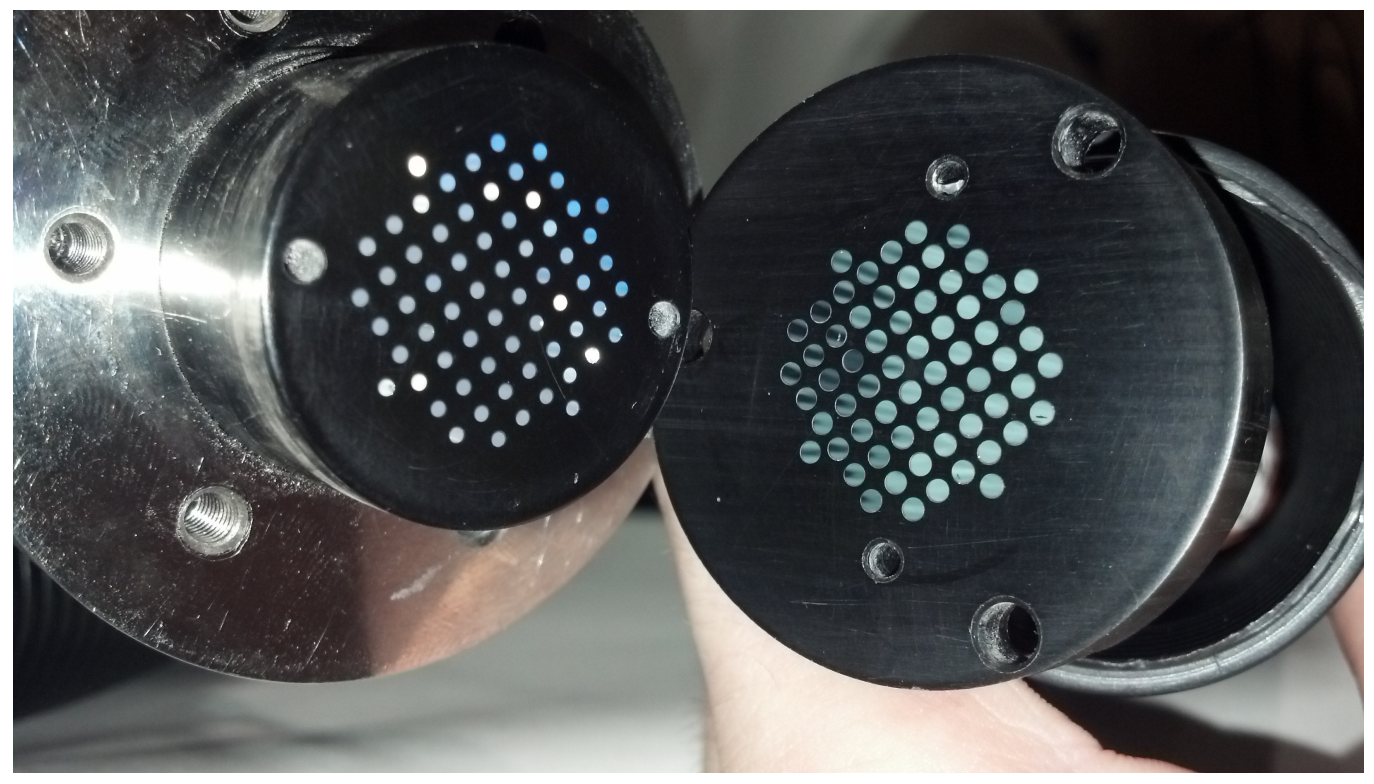

Figure 4.7: Two fiber separators. Scintillating fiber separator is on the left and optical fiber separator on the right.

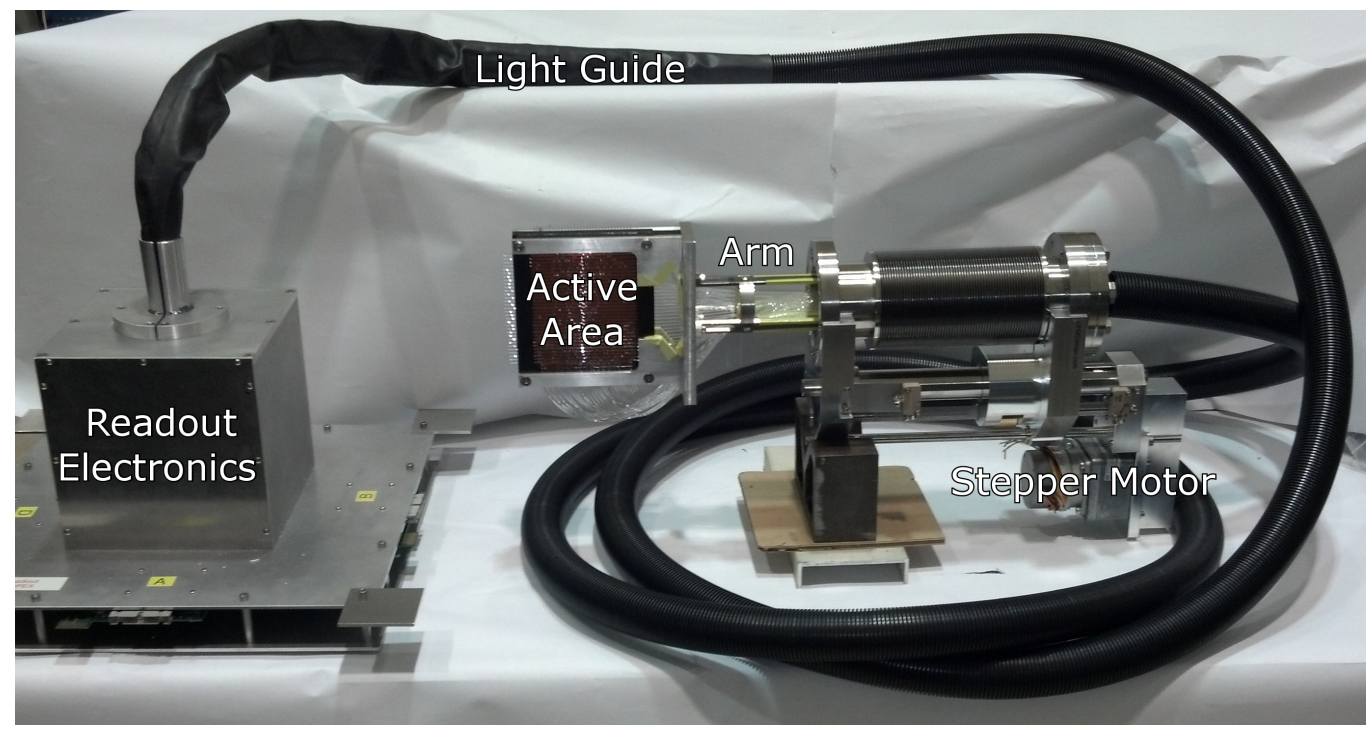

Figure 4.8: Complete setup of the SciFi detector. SciFi detector is connected to an mechanical arm driven by a stepper motor. Scinitllating fibers are coupled to clear optical fibers through two separators. The long 64 clear optical fibers guides the scintillation light to the readout electronics and maPMT. 
extension box, between target and the septum magnet. Figure 4.9 illustrates how it would look like when it is assembled in hall A. Initially, a sieve plate will be positioned in front of one of the SciFi detectors, to get a correspondence between the sieve hole locations and fibers. Then the sieve plate will be removed and the SciFi detector will measure electron and positron tracks. These data will be used to perform the optics calibration.

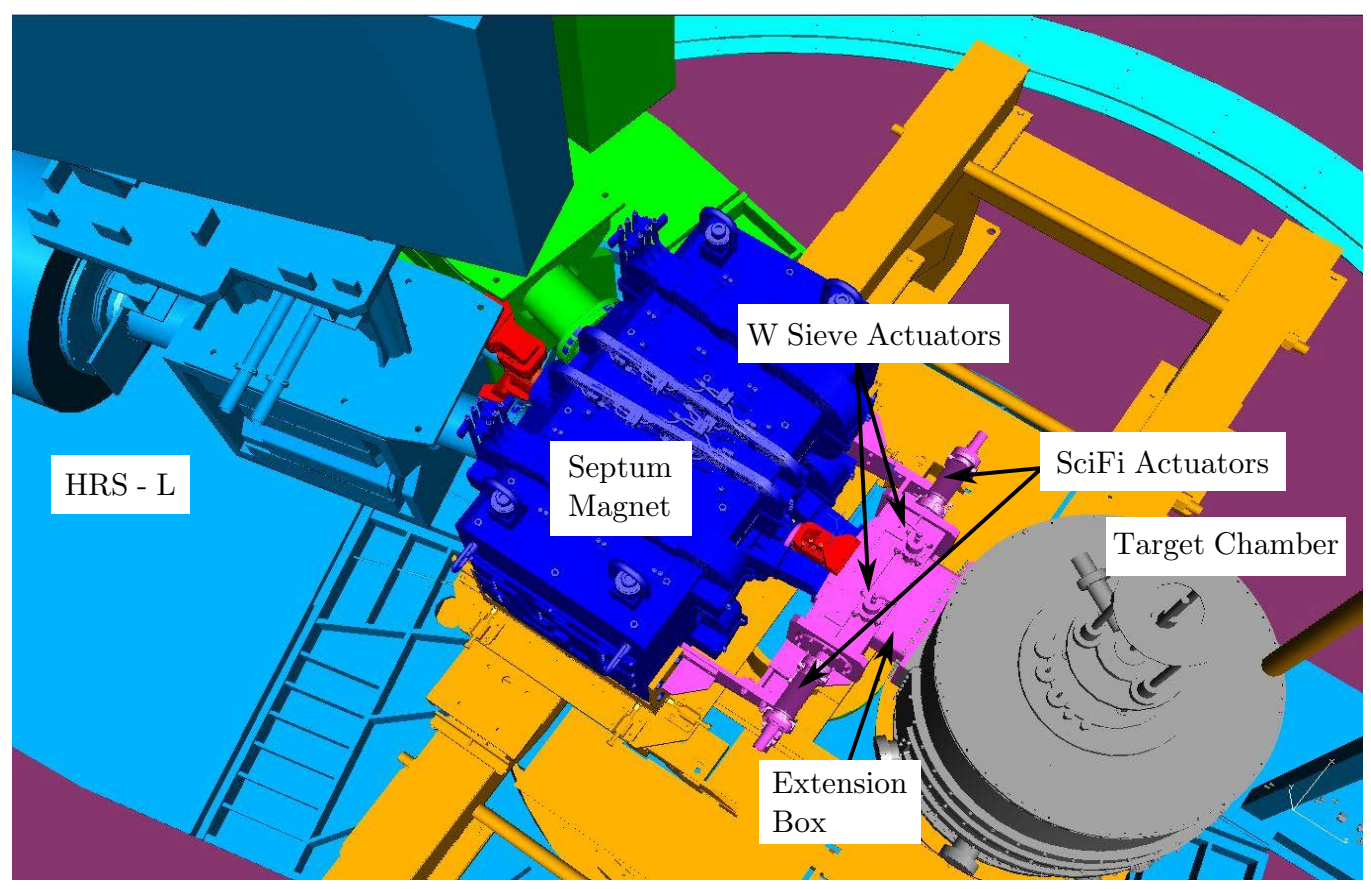

Figure 4.9: A 3D drawing of the hall A layout. Two SciFi detectors will be used, one for each HRS which are connected to the extension box through a vacuum feedthrough. SciFi actuators are stepper motors which will be used to move the SciFi in and out of the beam. 


\subsection{Experiment Setup to evaluate efficiency of the SciFi detector}

The efficiency of a scintillating fiber detector can be calculated using the amount of light captured in the PMT, or, the average number of photo-electrons produced at the photo-cathode.

To measure the average number of photo-electrons per event, a light-tight dark box was constructed to house the SciFi detector arm, a radiation source and a scintillating block trigger as illustrated in Figure 4.10. The SciFi was tested in a separate test setup where we used a 2-inch PMT instead of a maPMT. The SciFi separator was attached to a single 2-inch PMT which could detect light from all 64 fibers at once. Figure 4.11 and Figure 4.12 show two views of the inside and outside of the setup respectively. The output of this 2-inch PMT was connected to either a DAQ system or to an oscilloscope. The scintillating block trigger consisted of a single PMT attached via a light guide to a piece of scintillating material. The scintillating surface of the block trigger was placed parallel to the active area of the SciFi detector. A $5 \mu \mathrm{Ci}$ Ruthenium-106 which emits beta particles with a maximum energy of $39.4 \mathrm{keV}$ was used for the experiment. A Lecroy model HV4032A high voltage power supply was used to power the PMTs.

Figure 4.13 illustrates the block diagram for the SciFi test setup. When an electron deposited its energy in the scintillating fibers of the SciFi detector, it emitted scintillation light with its wavelength peaking at $\sim 437 \mathrm{~nm}$. The corresponding signal from the PMT was amplified using a PS 776 amplifier. The amplified signal was then sent to an Analog-to-Digital Converter (ADC) with a 100 ns delay line. A gate signal for the ADC was generated by the signal from the scintillating block trigger. When electron deposited energy in the scintillating block in the trigger, the corresponding 


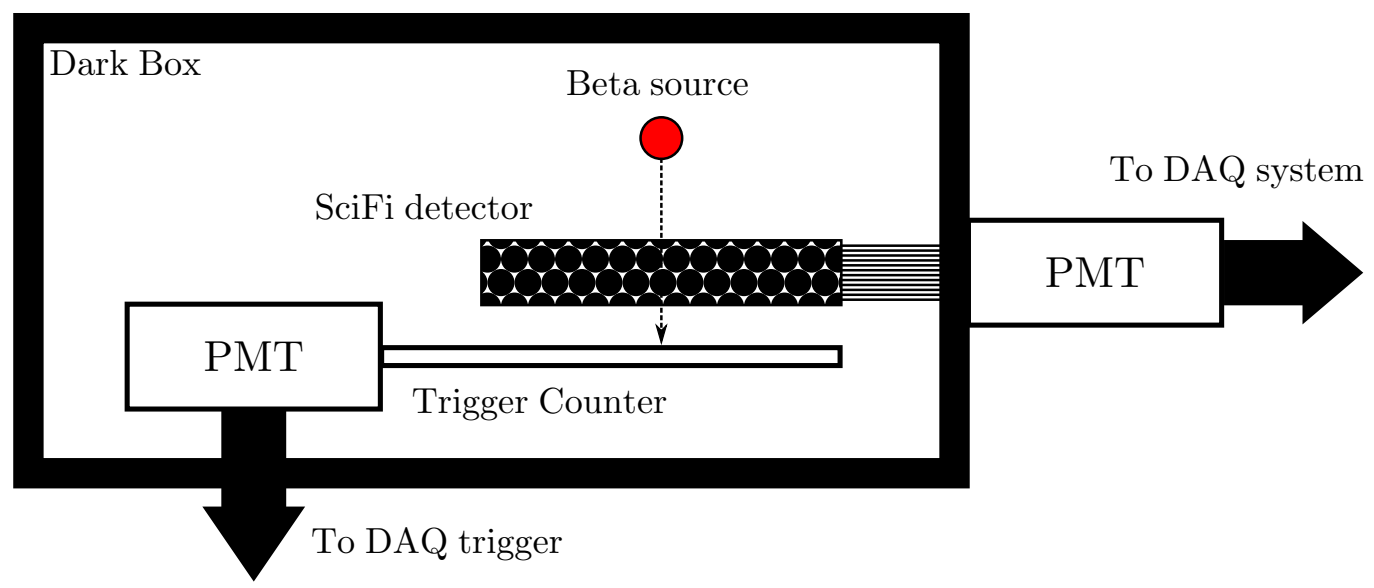

Figure 4.10: Custom light tight box constructed for the experiment. SciFi detector, Trigger counter and the radiation source were housed inside the dark box. A PMT was connected from outside of the box.

signal was sent to a PS 707 discriminator. The logic pulse from the discriminator was used to generate the gate signal for the ADC. Another 60 ns delay line was used for the trigger pulse. Only if the gate pulse and the signal from the SciFi coincided, the data were processed by the ADC. We used a CAEN Mod. V792 Charge-to-Amplitude Converter (QAC) as the ADC. A program based on CODA (CEBAF Online Data Acquisition) was used as the DAQ support software. The resultant ADC spectrum (PMT signal) was analyzed to obtain the average number of photo-electrons per each event that passed through the SciFi detector.

\subsection{Statistical model and fitting}

Figure 4.14 illustrates a pulse height spectrum obtained using the SciFi test setup. Electrons that reached the scintillating block trigger without interacting with the $\mathrm{SciFi}$, resulted in a pedestal peak in the spectrum. In other words, these are the events recorded by the DAQ system, when no signal from the SciFi was present. The remaining section of the spectrum is the events due to one or more photo-electrons. 


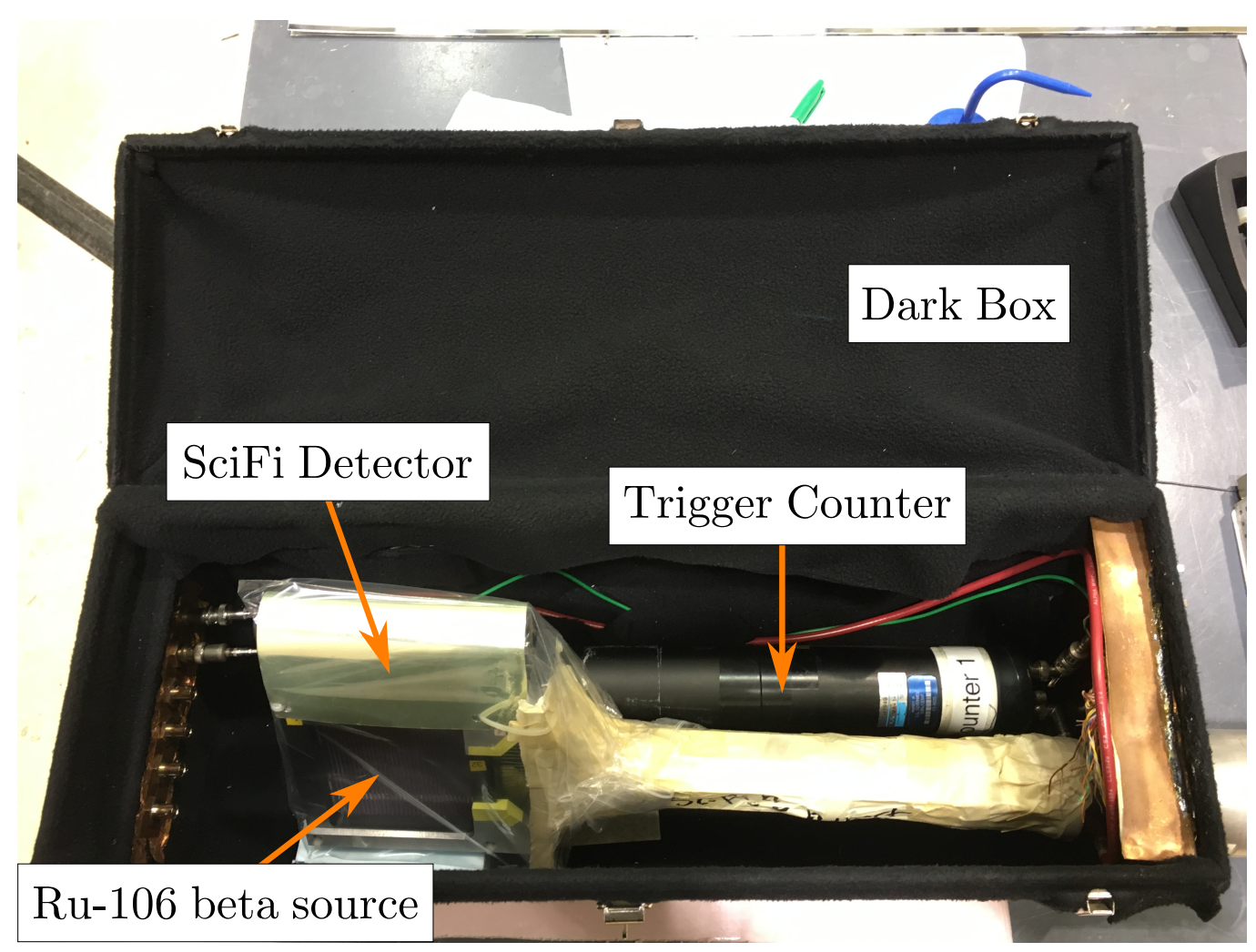

Figure 4.11: Inside of the dark box used for the experiment. Active area of the SciFi and the scintillating block of the trigger counter are placed parallel to each other. PMT of the trigger counter is inside the box. Radiation source was placed very close to the SciFi detector surface. 


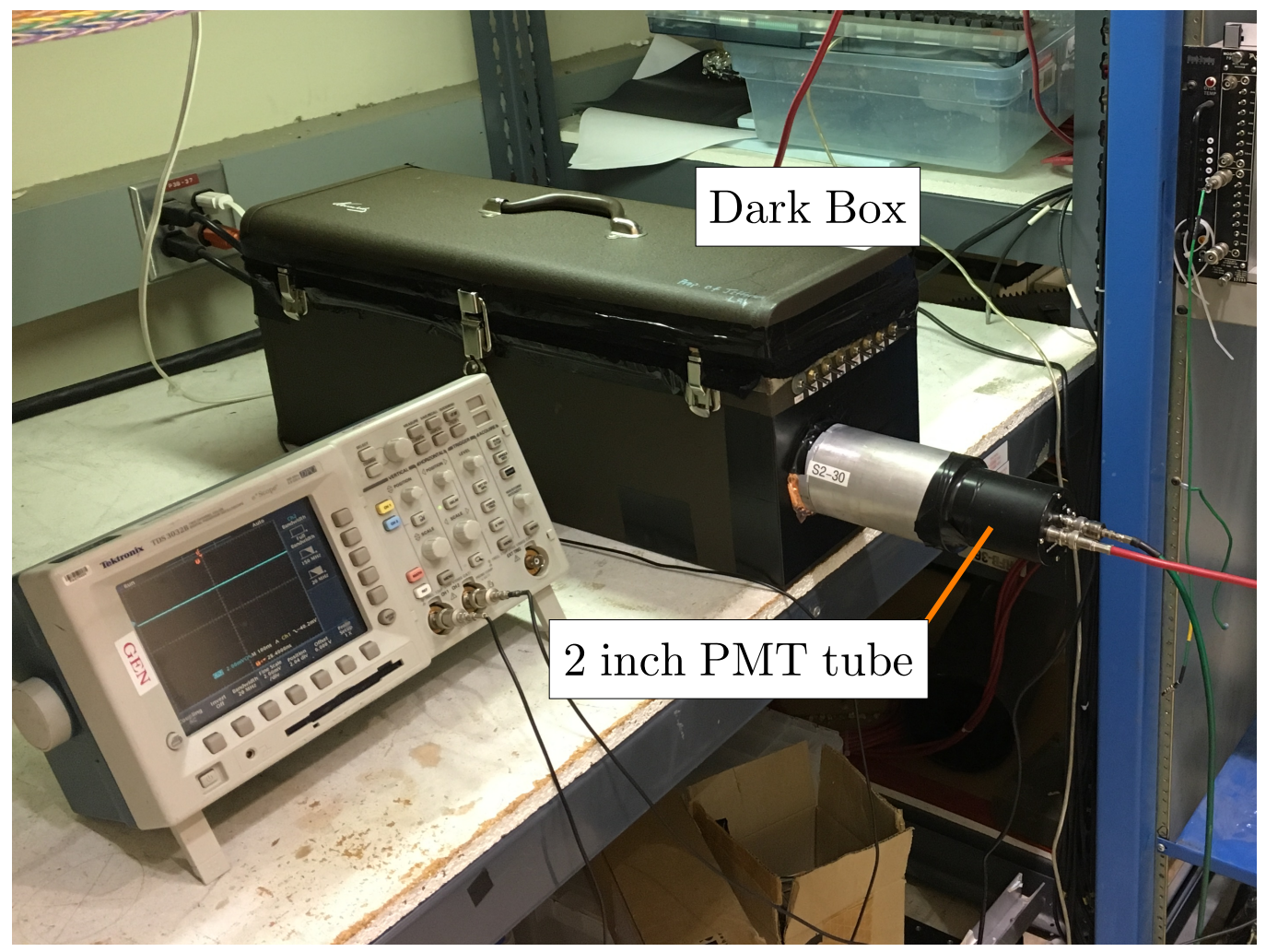

Figure 4.12: Outside of the dark box. An aluminum cylindrical structure was connected to the box from outside to hold the 2-inch PMT. The PMT and the scintillating fiber ends are touching inside the aluminum structure. 


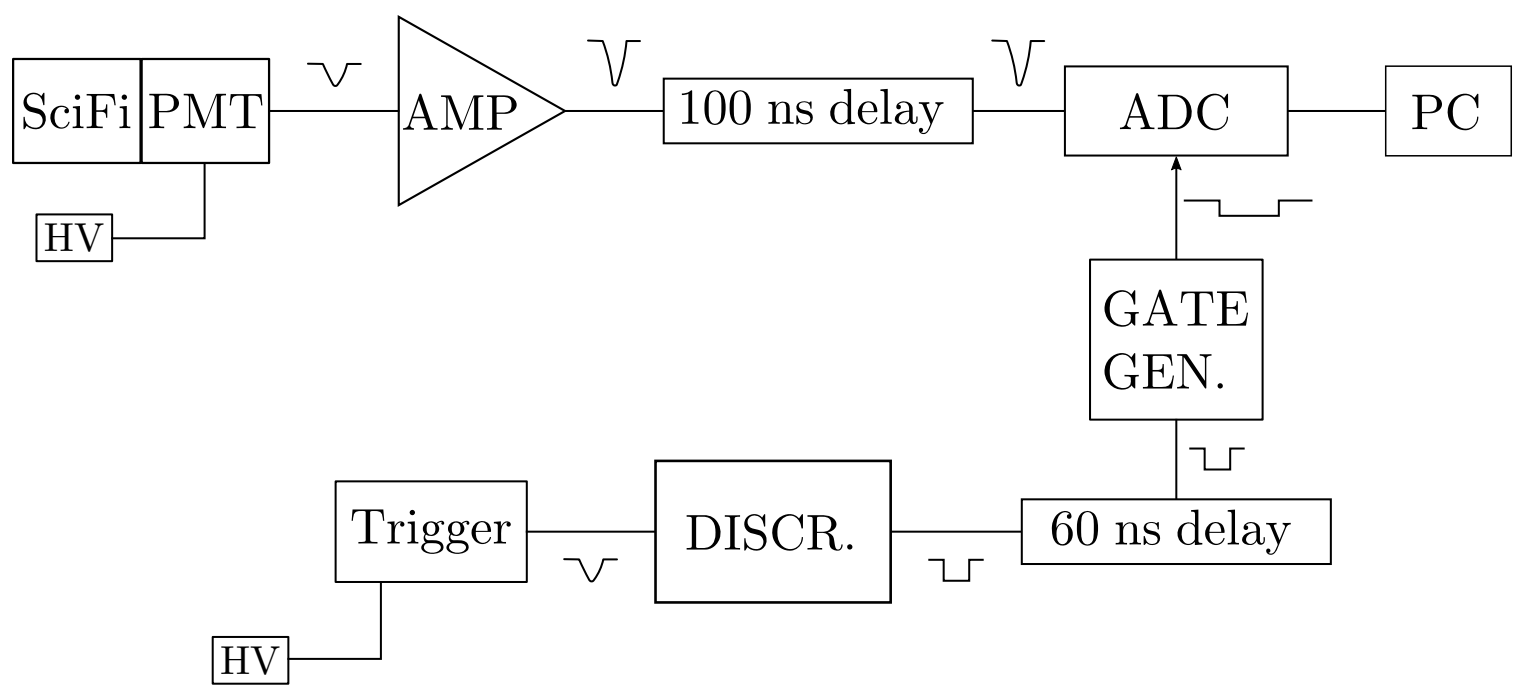

Figure 4.13: Block diagram of the experiment setup.

The pedestal peak can be described by a Gaussian function and the mean of the Gaussian function provides the ADC value that corresponds to the pedestal peak. This is the baseline from which all the spectrum measurements were read.

A photomultiplier tube (PMT) is an instrument which consists of two independent parts. The photo-cathode in which photons cause the photoemission of electrons and the dynode system which amplifies the initial charge. The number of photons hitting the photo-cathode follows a Poisson distribution. Due to quantum efficiency, only a fraction of the photons will be collected and converted into electrons. This process is a random binary process. Therefore, the distribution of the number of photo-electrons can be expressed as a convolution of Poisson and binary processes, which again results in a Poisson distribution:

$$
P(n ; \mu)=\frac{\mu^{n} e^{-\mu}}{n !}
$$

where $P(n ; \mu)$ is the probability of observing $n$ photoelectrons when the mean value 


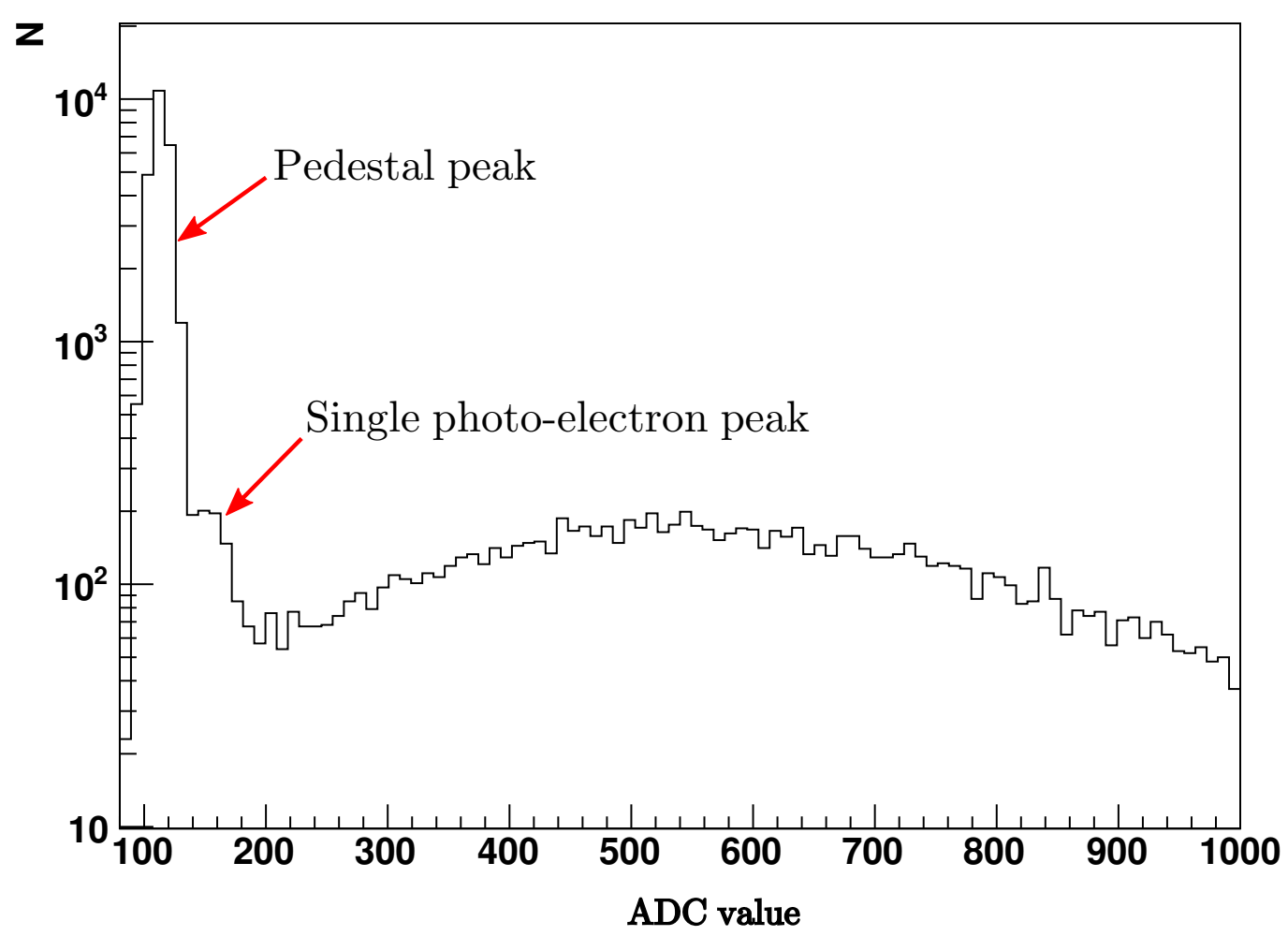

Figure 4.14: Example of a SciFi pulse height spectrum. The Pedestal peak is due to DAQ system getting triggered even when no signal is present. Rest of the spectrum is due to single or multiple photo-electron events. 
of photoelectrons is $\mu$. The response of a multiplicative dynode system to a single photoelectron, when the coefficient of secondary electron emission by the first dynode is large (>4), can be approximated by a Gaussian distribution [93]:

$$
G_{1}(q)=\frac{1}{\sigma_{1} \sqrt{2 \pi}} \exp \left(-\frac{\left(q-Q_{1}\right)^{2}}{2 \sigma_{1}^{2}}\right)
$$

where $q$ is the variable charge, $Q_{1}$ is the average charge at the PM output when one electron is collected by the first dynode, $\sigma_{1}$ is the corresponding standard deviation of the charge distribution. In the general case where $n$ photoelectrons are collected by the first dynode, the charge distribution is a convolution of $n$ single photoelectron cases [93] and is given by

$$
G_{n}(q)=\frac{1}{\sigma_{1} \sqrt{2 \pi n}} \exp \left(-\frac{\left(q-n Q_{1}\right)^{2}}{2 n \sigma_{1}^{2}}\right) .
$$

Here, we assume that the amplification process for each electron is mutually independent. We are now in a position to write down a function that describes the spectrum of an ideal noiseless PMT in which the pedestal is zero. The spectrum that we refer to here as $S_{\text {ideal }}(q)$, is simply a convolution of equation 4.11 and equation 4.13:

$$
\begin{aligned}
S_{\text {ideal }}(q) & =P(n ; \mu) \otimes G_{n}(q) \\
& =\sum_{n=1}^{\infty} \frac{\mu^{n} e^{-\mu}}{n !} \frac{1}{\sigma_{1} \sqrt{2 \pi n}} \exp \left(-\frac{\left(q-n Q_{1}\right)^{2}}{2 n \sigma_{1}^{2}}\right)
\end{aligned}
$$

\subsubsection{Evaluate the $\mu$ based on path length through a fiber}

The equation 4.15 represents a response function of a PMT when a mean number of photoelectrons, $\mu$, is collected by the first dynode. In the SciFi detector setup, however, $\mu$ can depend on the path an electron takes through the circular cross 
section of the scintillating fiber. As illustrated in the Figure 4.15, the number of scintillation photons produced in the fiber is proportional to the path length of a charged particle that traversed through the fiber, thus yielding a range of values for $\mu$.

$$
\mu(x)=\mu_{0} \sqrt{1-x^{2} / r^{2}}
$$

Here $\mu(x)$ was the mean number of photoelectrons collected by the first dynode, when a charged particle passed through a fiber at a distance, $x$, from the center of the fiber. The radius of the fiber was $r$. $\mu_{0}$ was the mean number of photo-electrons collected at the first dynode, when track passed through the center of the fiber.

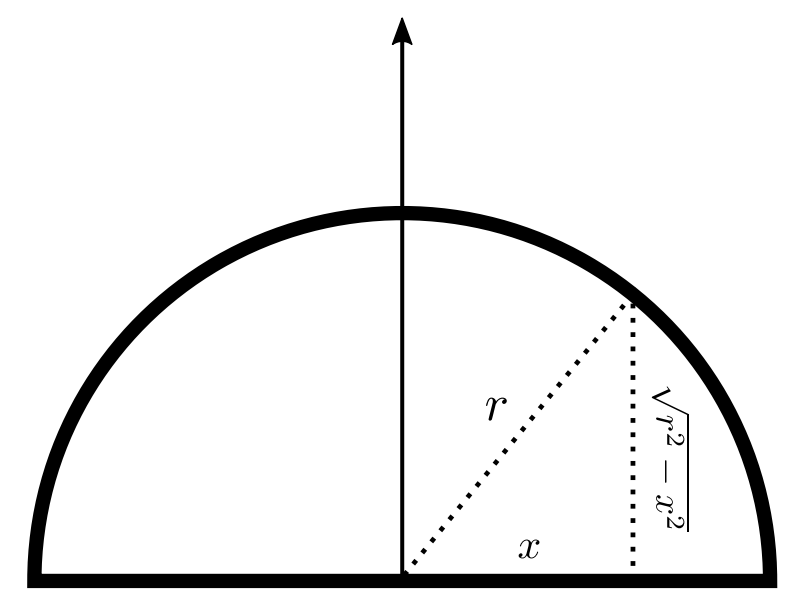

Figure 4.15: Upper half of the scintillating fiber with a circular cross section. A particle track passes the fiber vertically at distance $x$ from the center. The path length of the particle through the fiber is $\sqrt{r^{2}-x^{2}}$.

Now, the distribution of the number of photo-electrons in the PMT is given by $B\left(n ; \mu_{0}\right)$ which represents the normalized integral of $P(n ; \mu(x))$ over possible values of $x$. 


$$
\begin{aligned}
B\left(n ; \mu_{0}\right) & =\frac{1}{r} \int_{0}^{r} P(n ; \mu(x)) d x \\
& =\frac{1}{r} \int_{0}^{r} \frac{\mu(x)^{n} e^{-\mu(x)}}{n !} d x
\end{aligned}
$$

where $\mu(x)$ is given by equation 4.16 and $1 / r$ is a normalization factor. The response of an ideal PMT, taking the shape of the fiber into account, can thus be given by the following equation:

$$
\begin{aligned}
S_{\text {ideal }}(q) & =B\left(n ; \mu_{0}\right) \otimes G_{n}(q) \\
& =\sum_{n=1}^{\infty}\left(\frac{1}{r} \int_{0}^{r} \frac{\mu(x)^{n} e^{-\mu(x)}}{n !} d x\right) \frac{1}{\sigma_{1} \sqrt{2 \pi n}} \exp \left(-\frac{\left(q-n Q_{1}\right)^{2}}{2 n \sigma_{1}^{2}}\right) .
\end{aligned}
$$

To describe the pedestal, we need to add the following term,

$$
\left(\frac{1}{r} \int_{0}^{r} e^{-\mu(x)} d x\right) \frac{1}{\sigma_{0} \sqrt{2 \pi}} \exp \left(-\frac{\left(q-Q_{0}\right)^{2}}{2 \sigma_{0}^{2}}\right)
$$

where $Q_{0}$ is the average charge at the PMT output when no electrons are collected and $\sigma_{0}$ is the corresponding standard deviation of the charge distribution. For simplicity, the following notations are used;

$$
\begin{array}{r}
B_{n}\left(\mu_{0}\right)=\frac{1}{r} \int_{0}^{r} \frac{\mu(x)^{n} e^{-\mu(x)}}{n !} \\
G\left(n Q_{1}, \sqrt{n} \sigma_{1}\right)=\frac{1}{\sigma_{1} \sqrt{2 \pi n}} \exp \left(-\frac{\left(q-n Q_{1}\right)^{2}}{2 n \sigma_{1}^{2}}\right) \\
G\left(Q_{0}, \sigma_{0}\right)=\frac{1}{\sigma_{0} \sqrt{2 \pi}} \exp \left(-\frac{\left(q-Q_{0}\right)^{2}}{2 \sigma_{0}^{2}}\right)
\end{array}
$$

The equation 4.20 can be re-written as follows. 


$$
\begin{aligned}
S_{\text {ideal }}(x)= & B_{0}\left(\mu_{0}\right) G\left(Q_{0}, \sigma_{0}\right)+B_{1}\left(\mu_{0}\right) G\left(Q_{1}, \sigma_{1}\right) \\
& +B_{2}\left(\mu_{0}\right) G\left(2 Q_{1}, \sqrt{2} \sigma_{1}\right)+B_{3}\left(\mu_{0}\right) G\left(3 Q_{1}, \sqrt{3} \sigma_{1}\right)+\ldots
\end{aligned}
$$

As can be seen in the Figure 4.14, the pedestal value is the baseline for all the other photo-electron peaks. Therefore, equation 4.23 needs to be shifted right by an amount of $Q_{0}$.

$$
\begin{aligned}
S_{\text {ideal }}(x)= & B_{0}\left(\mu_{0}\right) G\left(Q_{0}, \sigma_{0}\right)+B_{1}\left(\mu_{0}\right) G\left(Q_{0}+Q_{1}, \sigma_{1}\right) \\
& +B_{2}\left(\mu_{0}\right) G\left(Q_{0}+2 Q_{1}, \sqrt{2} \sigma_{1}\right)+B_{3}\left(\mu_{0}\right) G\left(Q_{0}+3 Q_{1}, \sqrt{3} \sigma_{1}\right)+\ldots
\end{aligned}
$$

If $N_{h}$ is the number of events to hit the SciFi detector, then the true events recorded in the charge spectrum are given by $N_{h} S_{\text {ideal }}$. Events that missed the SciFi detector would appear as a part of the pedestal, and can be described using the expression, $N_{m} G\left(Q_{0}, \sigma_{0}\right)$. Here, $N_{m}$ is the number of events that missed the SciFi detector, but still hit the scintillating block trigger. This additional term needs to be added to equation 4.24. Thus we find that the

$$
\begin{aligned}
\text { FitFunction }= & \left(N_{m}+N_{h} B_{0}\left(\mu_{0}\right)\right) G\left(Q_{0}, \sigma_{0}\right) \\
& +N_{h} B_{1}\left(\mu_{0}\right) G\left(Q_{0}+Q_{1}, \sigma_{1}\right) \\
& +N_{h} B_{2}\left(\mu_{0}\right) G\left(Q_{0}+2 Q_{1}, \sqrt{2} \sigma_{1}\right) \\
& +N_{h} B_{3}\left(\mu_{0}\right) G\left(Q_{0}+3 Q_{1}, \sqrt{3} \sigma_{1}\right)+\ldots .
\end{aligned}
$$

Equation 4.25 is the fitting function that was used to fit the charge spectrum of the SciFi detector. The fitting program adjusted the fitting parameters until it 
minimized the $\chi^{2} /$ ndf. Calculating $B_{n}\left(\mu_{0}\right)$ (equation 4.18) for a particular value of the fitting parameter $\mu_{0}$ requires an integration, doing so as part of the fitting process was time consuming and required considerable processing power. Therefore, we followed an analytical method where we calculated $B_{n}\left(\mu_{0}\right)$ for a discrete set of values of $\mu_{0}$ outside the fitting program. Figure 4.16 shows these calculations for $n=3$ and $n=10$ along with a spline-function fit, which could be used to predict $B_{n}\left(\mu_{0}\right)$ for any arbitrary value of $\mu_{0}$.
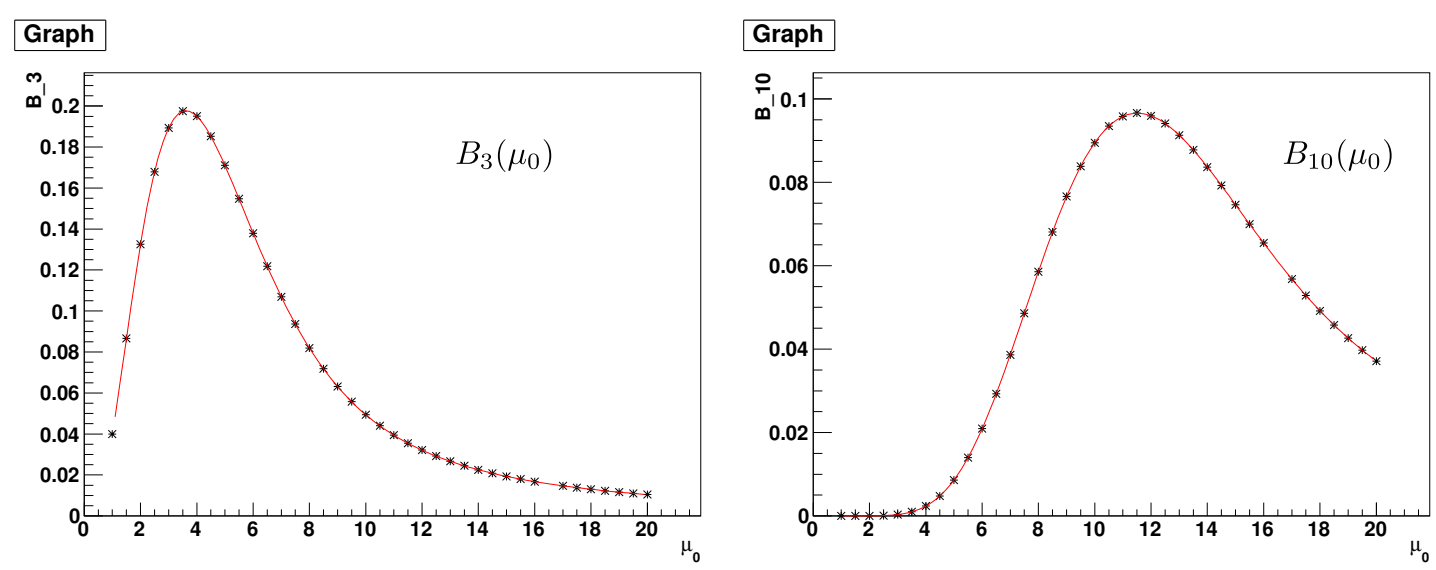

Figure 4.16: The distribution of photoelectrons in the PMT (used with the SciFi) is given by the probability function $B_{n}\left(\mu_{0}\right)$. Calculations of $B_{n}\left(\mu_{0}\right)$ for only $n=3$ and $n=10$ as a function of $\mu_{0}$ are shown in the figure.

Figure 4.17 illustrates the fitting of a charge spectrum from the SciFi with the fitting function described in equation 4.25. As can be seen in the figure 4.17, the fitting function had difficulty in fitting the single photoelectron peak. There could be accidental events which contributed to additional photoelectron events. We introduced two other parameters, $a c c 1$ and $a c c 2$, to describe these additional events in the single photoelectron and two photoelectron peaks respectively. 


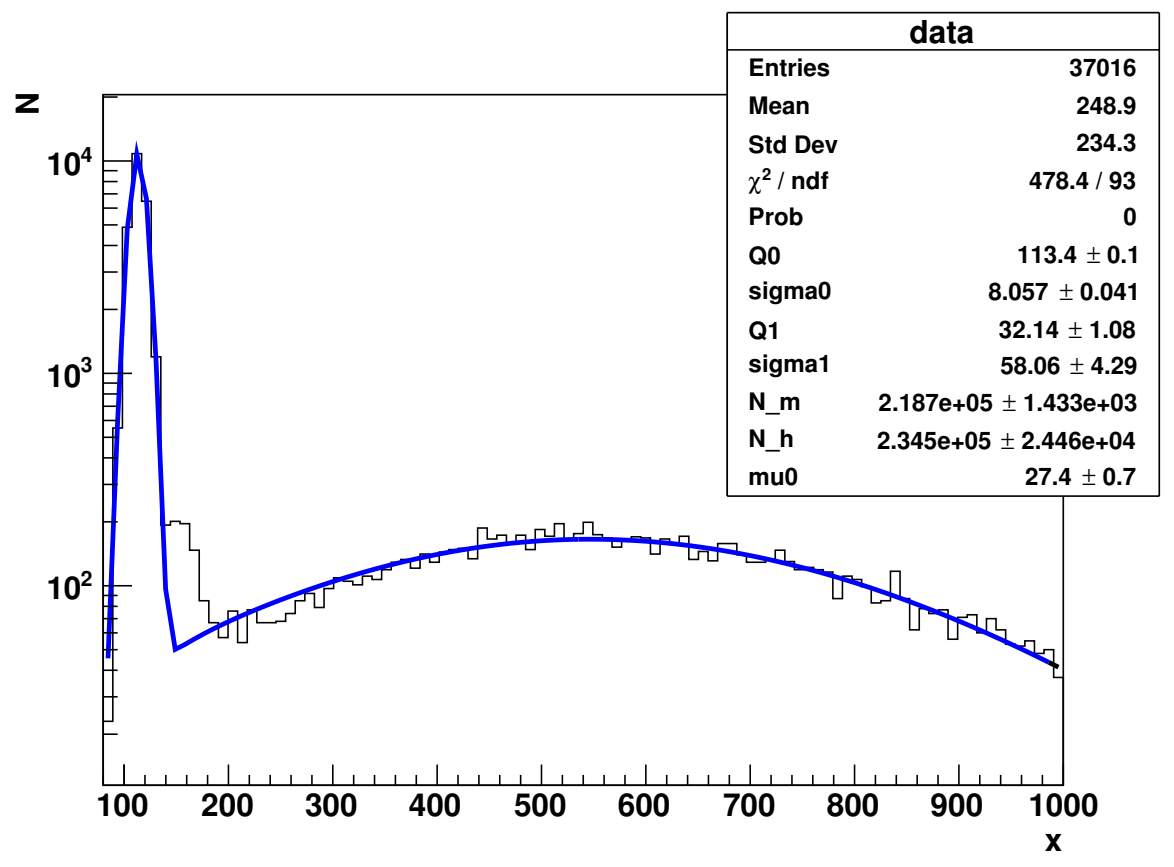

Figure 4.17: Pulse height spectrum of SciFi detector when 2-inch PMT was connected directly to the dark box. The equation 4.25 was used to fit the distribution. This has a poor fit to the single photo-electron peak. 


$$
\begin{aligned}
\text { FitFunction }= & \left(N_{m}+N_{h} B_{0}\left(\mu_{0}\right)\right) G\left(Q_{0}, \sigma_{0}\right) \\
& +\left(N_{h} B_{1}\left(\mu_{0}\right)+\operatorname{acc} 1\right) G\left(Q_{0}+Q_{1}, \sigma_{1}\right) \\
& +\left(N_{h} B_{2}\left(\mu_{0}\right)+\operatorname{acc} 2\right) G\left(Q_{0}+2 Q_{1}, \sqrt{2} \sigma_{1}\right) \\
& +N_{h} B_{3}\left(\mu_{0}\right) G\left(Q_{0}+3 Q_{1}, \sqrt{3} \sigma_{1}\right)+\ldots
\end{aligned}
$$

Equation 4.26 shows the new fitting function and Figure 4.18 illustrates the fit to the histogram. We observe a clear improvement, but $\chi^{2} /$ ndf is still too large.

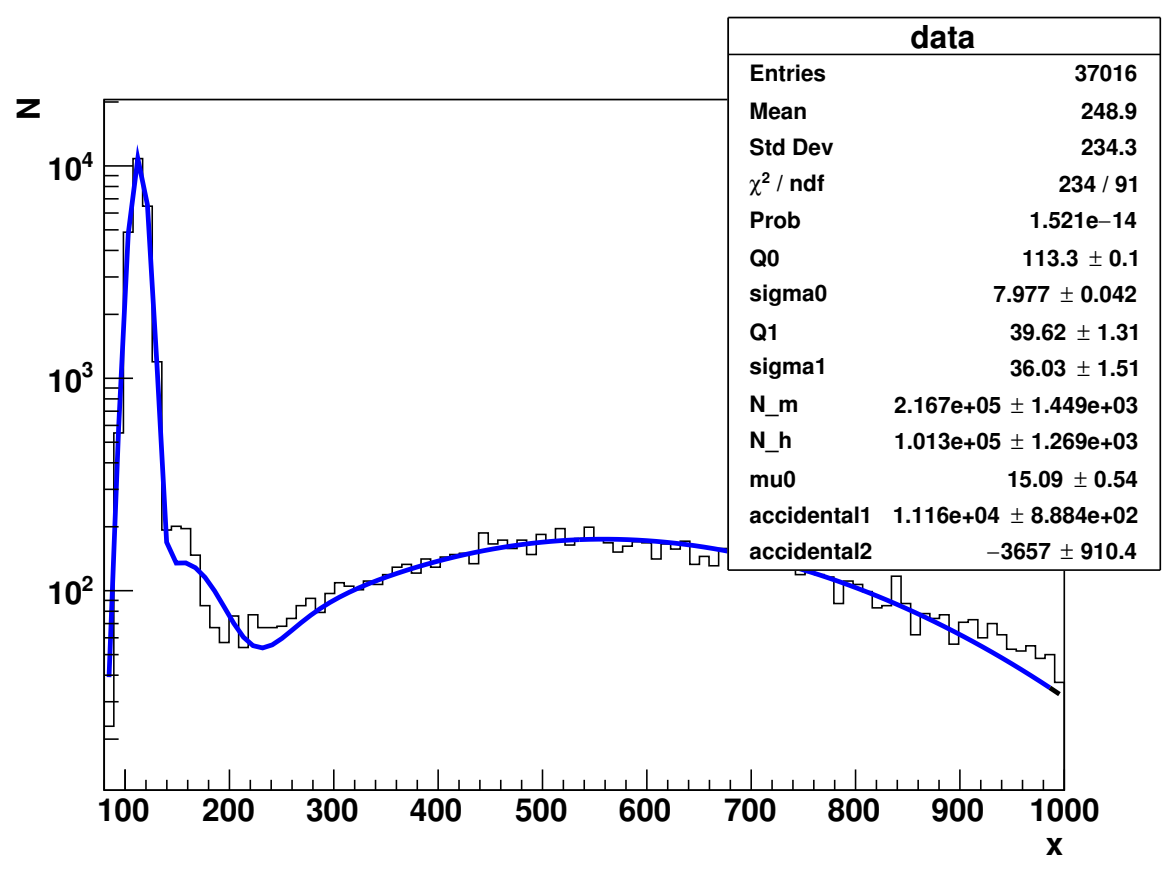

Figure 4.18: Pulse height spectrum of SciFi detector when 2-inch PMT was connected directly to the dark box. The equation 4.26 was used to fit the distribution. Fit to the single photo-electron peak is improved by introducing 2 more parameters acc 1 and $a c c 2$ to represent accidental events. 


\subsubsection{Evaluate $\mu$ based on true detector response}

In the previous method, the geometric effect of the SciFi was approximated by considering the track length of electrons through a single fiber. However, in the actual SciFi detector, there were two layers of scintillating fibers and they were closely positioned. Therefore, the true response of the SciFi detector should be different from that of a single fiber. In this method, we considered a more realistic geometric effect by simulating the true response of the SciFi by calculating energy deposition in the SciFi detector. The Geant4 Monte-Carlo toolkit was used to develop a model of the active area of the SciFi detector, which included 64 scintillating fibers. About $1 \times 10^{6}$ electrons of $1 \mathrm{GeV}$ of energy were generated and incident on the SciFi detector. Figure 4.19 shows an image of the Geant 4 model of the SciFi detector and its simulation. Figure 4.20 shows the histogram generated by binning the events over total energy deposited in all 64 fibers.

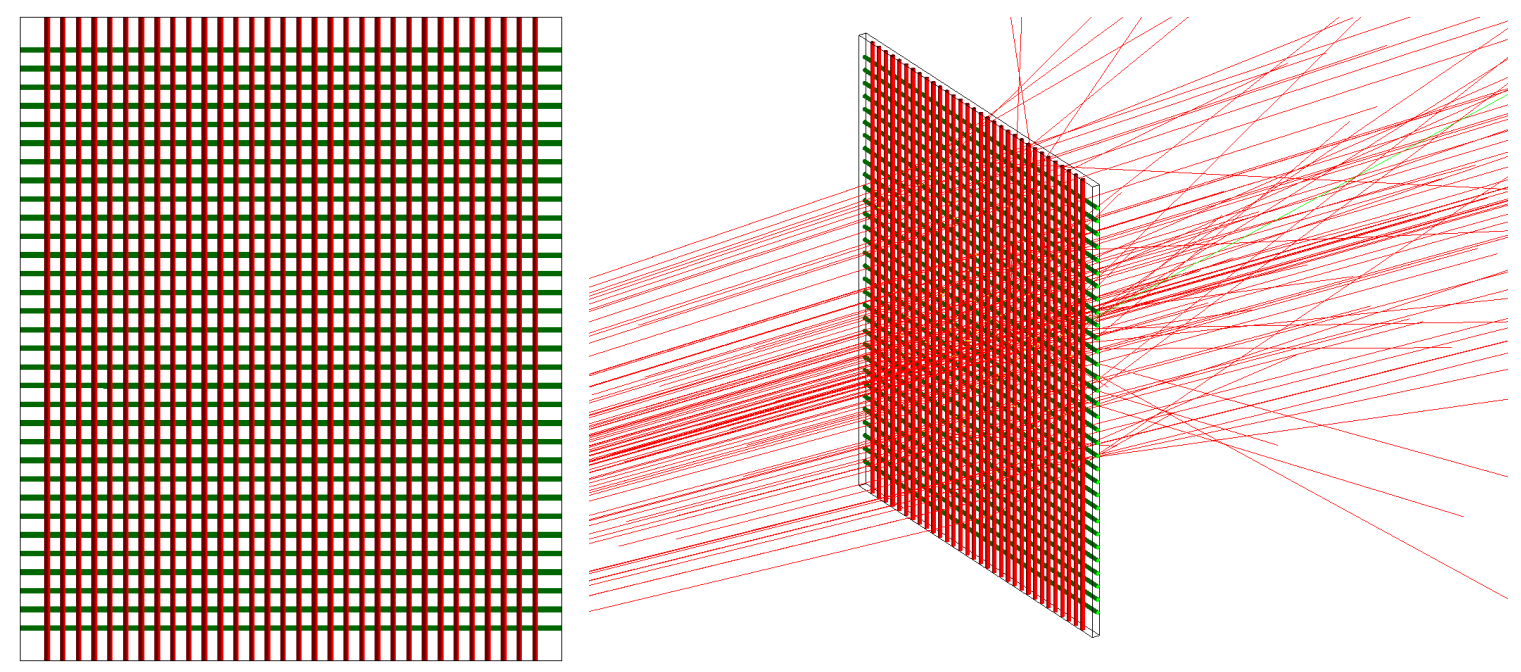

Figure 4.19: The energy deposition in scintillating fibers of SciFi detector was simulated using Geant4 package. The left image shows the 2-layer arrangement of scintillating fibers. The image on the right shows a snapshot taken during the simulation. 


\section{Pulse height spectrum for SciFi detector}

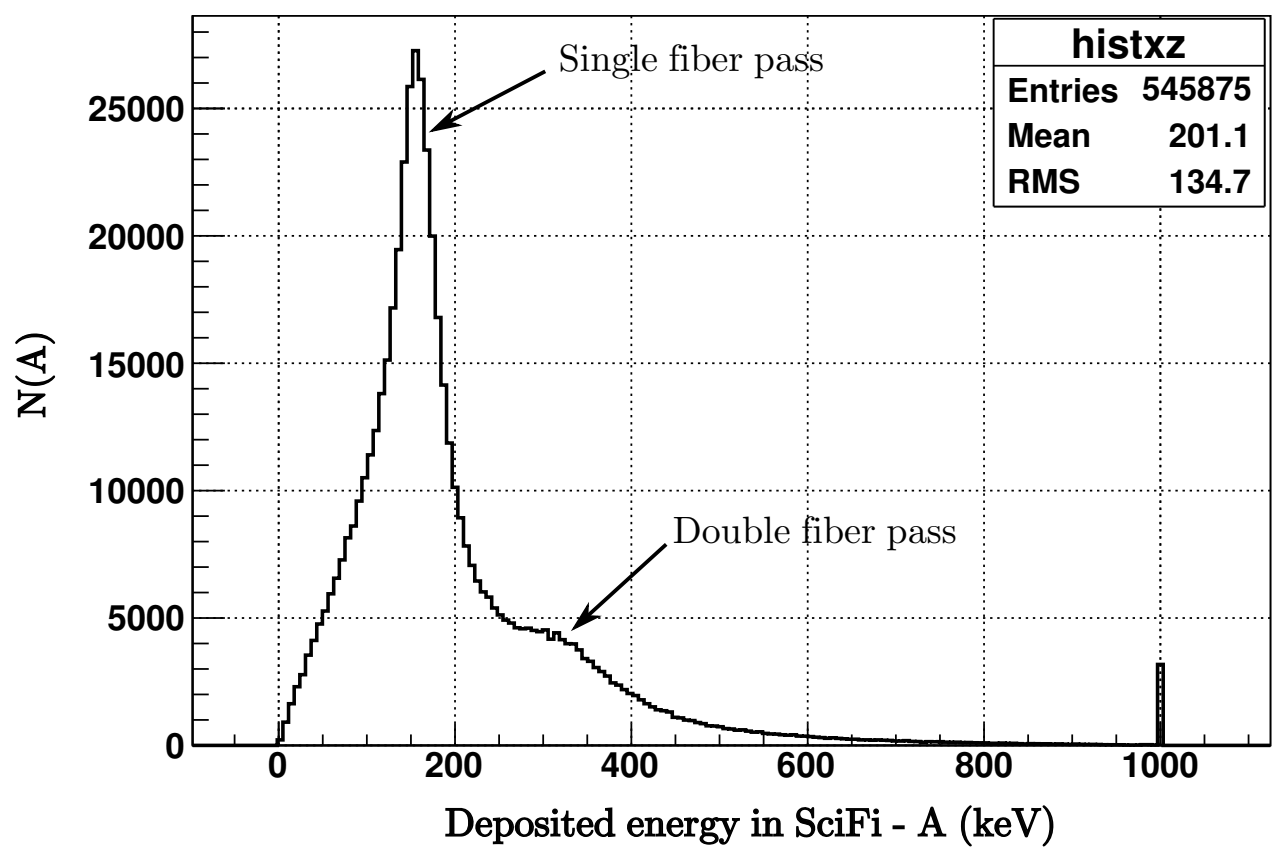

Figure 4.20: In the histogram, events are binned by the total energy deposited in all 64 fibers. The peak around $160 \mathrm{keV}$ of deposited energy (single fiber pass) resulted from electrons passing through a single fiber. The other peak around $300 \mathrm{keV}$ (double fiber pass) is due to electrons passing through a crossing of two fibers.

In this method, the distribution of the number of photoelectrons in the PMT is given by,

$$
\begin{gathered}
B_{n}\left(A_{0}\right)=\frac{\sum_{A} P_{n}(\mu) N(A)}{\sum_{A} N(A)} \\
\mu=\frac{A}{A_{0}},
\end{gathered}
$$

which represents the normalized sum of $P_{n}(\mu)$ weighted by the number of events, $N(A)$, in the histogram (Figure 4.20) for different values of $A$. Here $\mu$ is the mean number of photoelectrons collected by the first dynode of the PMT, $A$ represents the 
deposited energy in the SciFi and $A_{0}$ represents the energy deposited in the SciFi which resulted in a single photoelectron in the PMT.

Similar to the analytical method we discussed in section 4.6.1, that was used to reduce the processing time, we calculated $B_{n}\left(A_{0}\right)$ for a discrete set of values of $A_{0}$ outside the fitting program. Figure 4.21 shows these calculations for $n=0$ to $n=5$ along with a spline-function fit, which could then be used to predict $B_{n}\left(A_{0}\right)$ for any arbitrary value of $A_{0}$. We calculated $B_{n}\left(A_{0}\right)$ values up to $n=39$ for the final calculation. The fitting function shown in the equation 4.26 can still be used in this method after replacing $B_{n}\left(\mu_{0}\right)$ with the function $B_{n}\left(A_{0}\right)$. Thus, we find that the

$$
\begin{aligned}
\text { FitFunction }= & \left(N_{m}+N_{h} B_{0}\left(A_{0}\right)\right) G\left(Q_{0}, \sigma_{0}\right) \\
& +\left(N_{h} B_{1}\left(A_{0}\right)+\operatorname{acc} 1\right) G\left(Q_{0}+Q_{1}, \sigma_{1}\right) \\
& +\left(N_{h} B_{2}\left(A_{0}\right)+a c c 2\right) G\left(Q_{0}+2 Q_{1}, \sqrt{2} \sigma_{1}\right) \\
& +N_{h} B_{3}\left(A_{0}\right) G\left(Q_{0}+3 Q_{1}, \sqrt{3} \sigma_{1}\right)+\ldots .
\end{aligned}
$$

As shown in the Figure 4.22, calculating the distribution of photoelectrons based on the energy deposition in the SciFi gave a better fit to the charge spectrum. The best fit value for $A_{0}$ was calculated as $\sim 10 \mathrm{keV}$. As can be seen in the histogram (Figure 4.20), the energy deposition in the SciFi when an electron passed through just a single fiber (single pass) was $\sim 160 \mathrm{keV}$. Therefore, the mean number of photoelectrons collected by the first dynode during a single pass became $\sim 16$.

Figure 4.23 shows the crossing of 2 horizontal fibers and 2 vertical fibers in the SciFi detector. These types of crossing occur repeatedly throughout the sensitive area of the SciFi detector. Electrons can hit on a scintillating fiber and this is indicated by $N_{h}$ in the fitting function shown in the equation 4.29 . There is an empty space at 

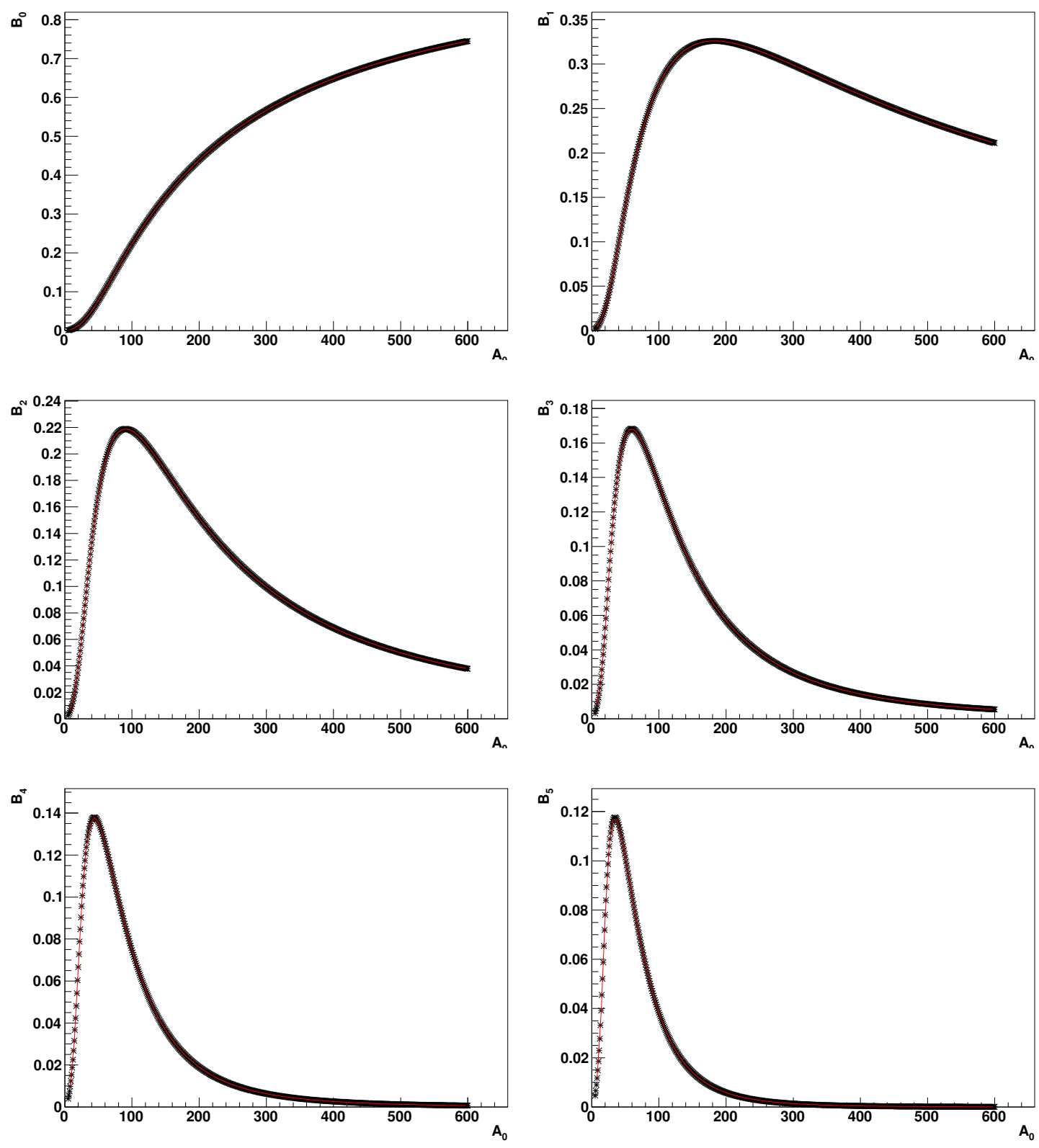

Figure 4.21: The distribution of photoelectrons in the PMT (used with the SciFi) is given by the probability function $B_{n}\left(A_{0}\right)$. Calculations of $B_{n}\left(A_{0}\right)$ for only $n=0$ to $n=5$ as a function of $A_{0}$ are shown in the figure. 


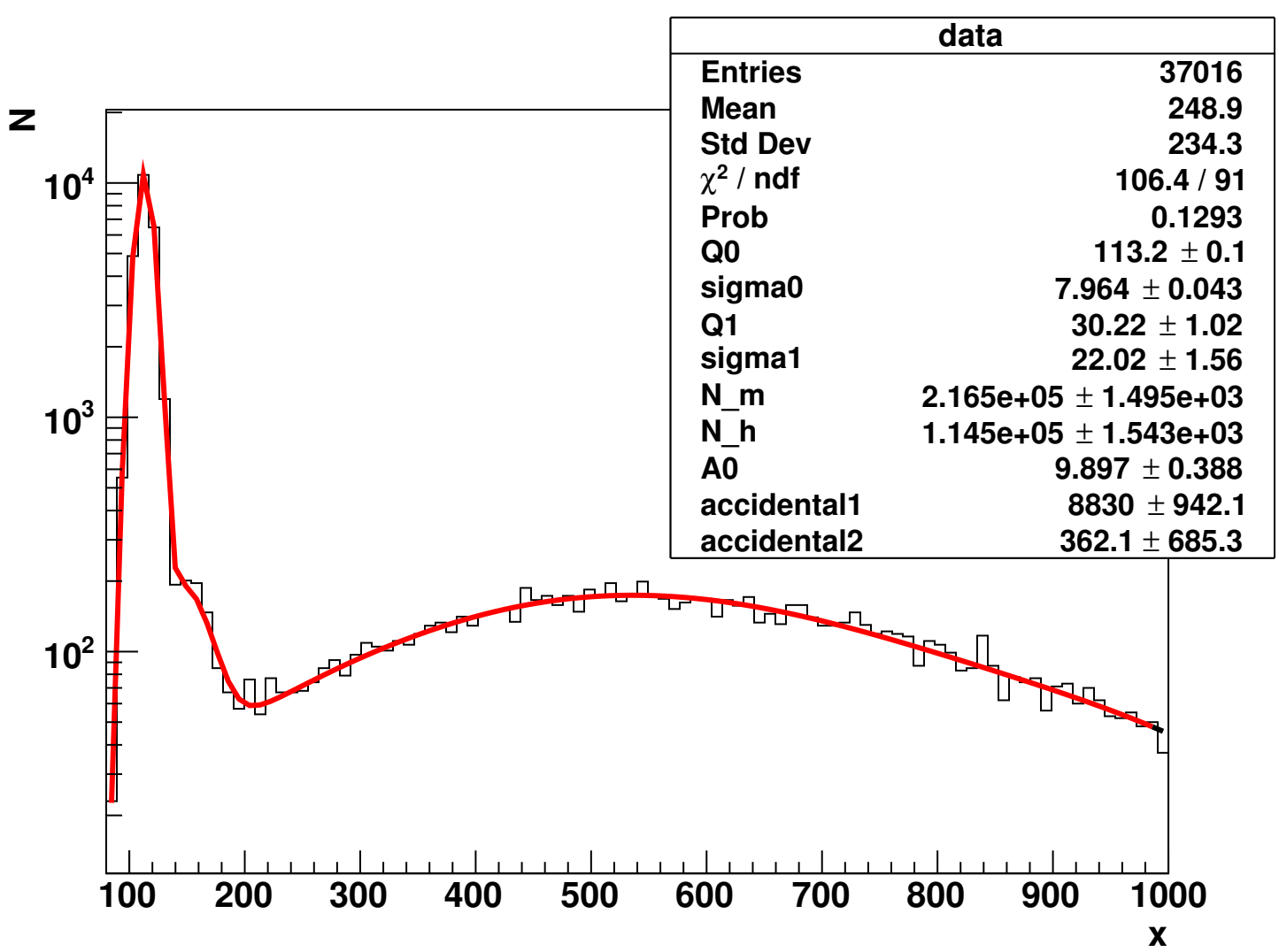

Figure 4.22: Pulse height spectrum of SciFi detector when 2-inch PMT was connected directly to the dark box. The equation 4.29 was used to fit the distribution. The probability function $B_{n}\left(\mu_{0}\right)$ was calculated based on the true response of the SciFi detector.

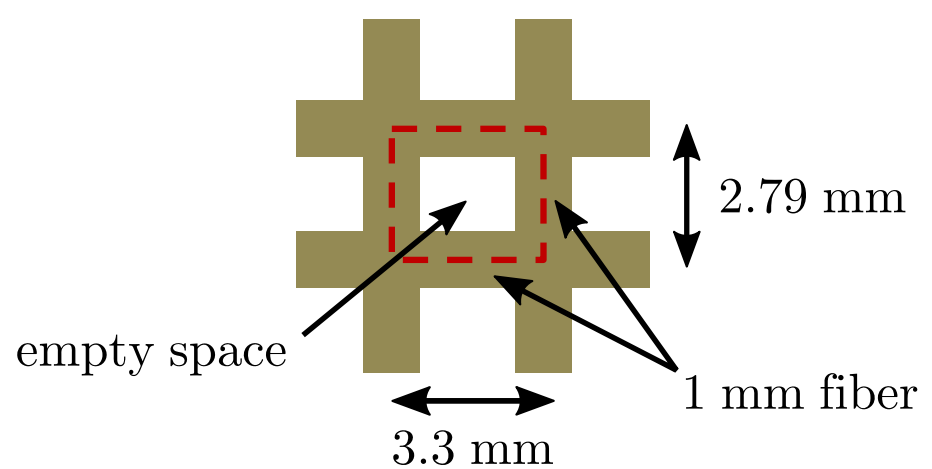

Figure 4.23: A repeating element of the active area of the SciFi detector. This was used to calculate the ratio of the area of fiber to the area of empty spaces. 
the center of the 4 fibers. Electrons that passed through these empty spaces typically missed the detector, but hit the scintillating block trigger. These events thus get collected in the pedestal of the histogram. These events are indicated by $N_{m}$ in the fitting function. The ratio $N_{h} / N_{m}$ based on the best fit values of the charge spectrum shown in Figure 4.22 was $\sim 1 / 2$. This should be equal to the area of the fibers to the area of the empty spaces, which was calculated as $\sim 1 / 0.8$. To reduce the extra events in the pedestal, we replaced the scintillating block trigger with another scintillating block trigger with much smaller area and thickness. Figure 4.24 shows the fit to the histogram, acquired using the setup with the smaller scintillating block trigger. With this new configuration, the mean number of photoelectrons collected by the first dynode during a single pass was $\sim 12$. The fitted ratio $N_{h} / N_{m}$ had an improved value of $\sim 1 / 1.7$. We believe the excess pedestal events were due to the photons triggering the DAQ system.

For the above measurements we removed the $\sim 3 \mathrm{~m}$ long optical light guide which connected the SciFi to a maPMT. Figure 4.8 shows the complete setup that will be used in the JLab Hall A. Light guides with a length of a few meters are needed to keep the maPMT away from the magnets as well as to protect the device from high radiation fields in the Hall A. Therefore, it was important to calculate the optical loss when light traverse through this long optical cable. We disassembled the 2-inch PMT from the dark box and connected one end of the cable to the scintillating fiber ends. The other end of the cable was connected to the 2-inch PMT. Figure 4.25 shows the fitted histogram obtained with the new setup. Based on the value $A_{0}$, there were $\sim 4$ photoelectrons produced in the PMT for each event in the detector. The attenuation in the long light guide is thus estimated to be $\sim 75 \%$. In the histogram, there is a high energy tail which is difficult to fit and the source of these extra events is as yet unclear. 


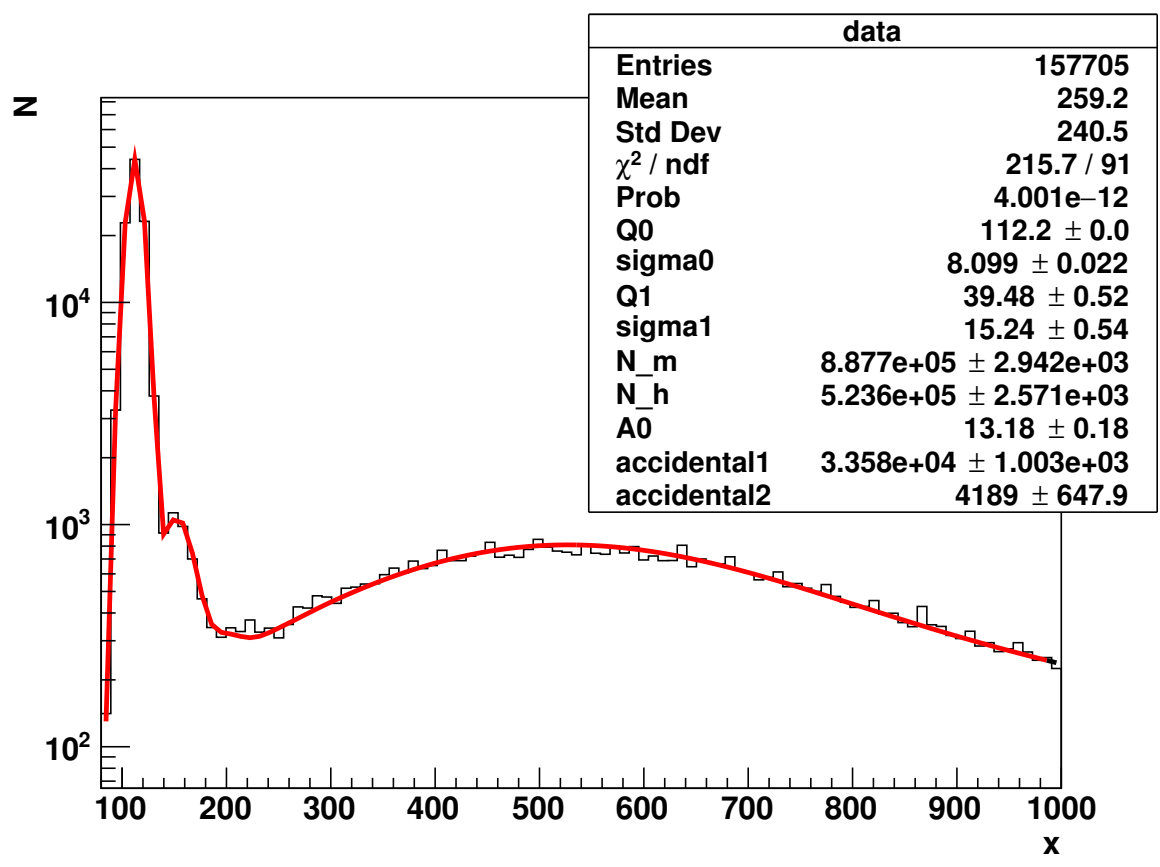

Figure 4.24: Pulse height spectrum of SciFi detector when 2-inch PMT was connected directly to the dark box. We used a trigger counter with smaller area and thickness compared to the setup which produced the spectrum in figure 4.22. 


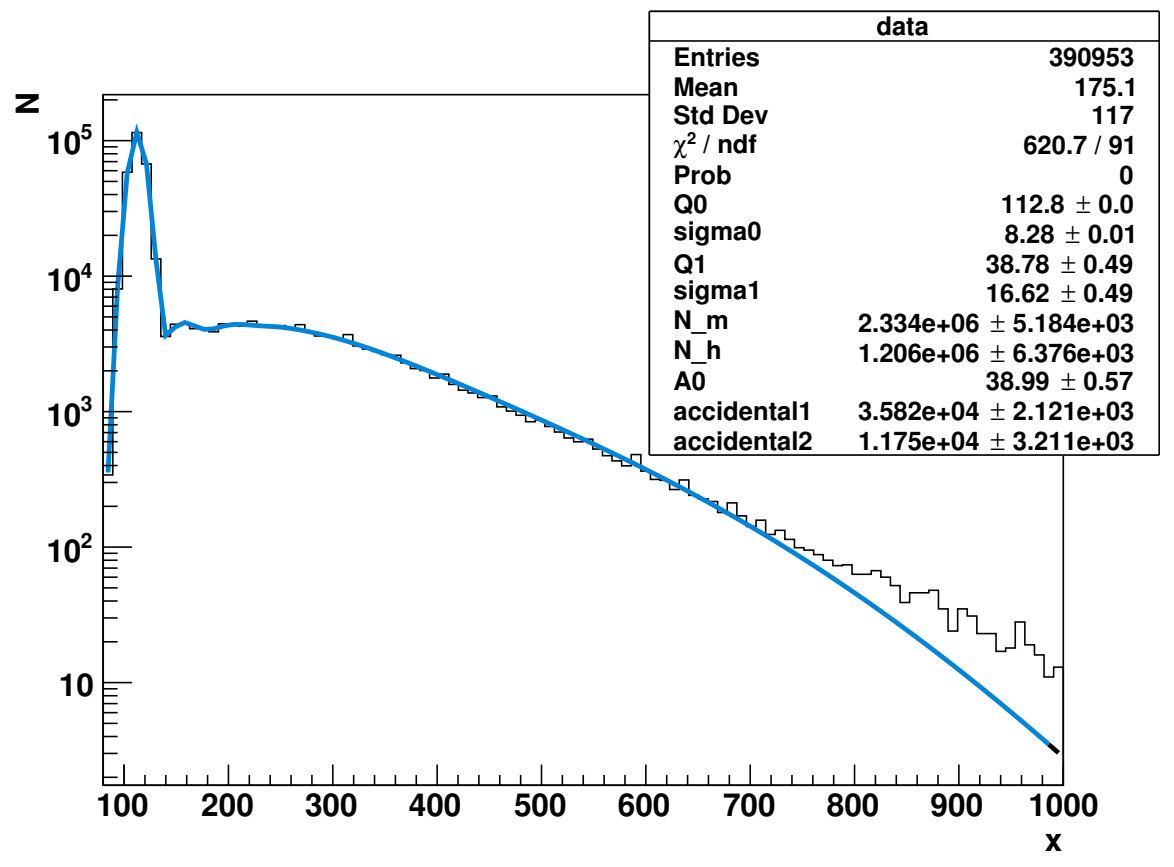

Figure 4.25: Pulse height spectrum of SciFi detector when 2-inch PMT was connected via the long optical cable. The source of extra events in the high energy tail is unknown. 


\subsection{Expected counting rate in SciFi}

In 2011, Neil Goeckner-Wald and Bogdan Wojtsekhowski proposed a plan to perform the optics calibration using the SciFi detector [72]. Some of the calculations in this plan were re-evaluated with the new information about the SciFi detector. They proposed to use a Carbon (C) target with a thickness of $50 \mathrm{mg} / \mathrm{cm}^{2}$ and a Tungsten (W) target with a thickness of $28.9 \mathrm{mg} / \mathrm{cm}^{2}$. An electron beam of $2.2 \mathrm{GeV}$ was proposed for the calibration run. The SciFi detector will be placed at about $1 \mathrm{~m}$ downstream of the target and the central angle of the SciFi detector will be $5^{\circ}$ from the beam direction.

Geant4 was used to simulate the expected rate of particles from the proposed targets. Figures 4.26 and 4.27 illustrate the rates of electrons produced in the $\mathrm{W}$ and $\mathrm{C}$ targets respectively. Table 4.1 summarizes the event rates at the angle of $5^{\circ}(0.08 \mathrm{rad})$

Table 4.1: Expected production of particles in a $\mathrm{W}$ target of $28.9 \mathrm{mg} / \mathrm{cm}^{2}$ and a $\mathrm{C}$ target of $50 \mathrm{mg} / \mathrm{cm}^{2}$ at $5^{\circ}$ angle with respect to the beam direction. A $2.2 \mathrm{GeV}$ electron beam was used with both targets.

\begin{tabular}{|c|c|c|c|}
\cline { 2 - 4 } \multicolumn{1}{c|}{} & \multicolumn{3}{|c|}{$\frac{1}{\mathrm{~N}_{\mathrm{e}}} \frac{\mathrm{dN}}{\mathrm{dE}}\left(\right.$ electron $\left.^{-1} \mathrm{sr}^{-1}\right)$} \\
\hline Target & $e^{-}$ & $e^{+}$ & $\gamma$ \\
\hline $\mathrm{W}, 28.9 \mathrm{mg} / \mathrm{cm}^{2}$ & $2 \times 10^{-3}$ & $1 \times 10^{-5}$ & $4 \times 10^{-5}$ \\
\hline $\mathrm{C}, 50.0 \mathrm{mg} / \mathrm{cm}^{2}$ & $2 \times 10^{-3}$ & $1 \times 10^{-4}$ & $7 \times 10^{-5}$ \\
\hline
\end{tabular}

The expected counting rate in a single scinitllating fiber for the proposed calibration 


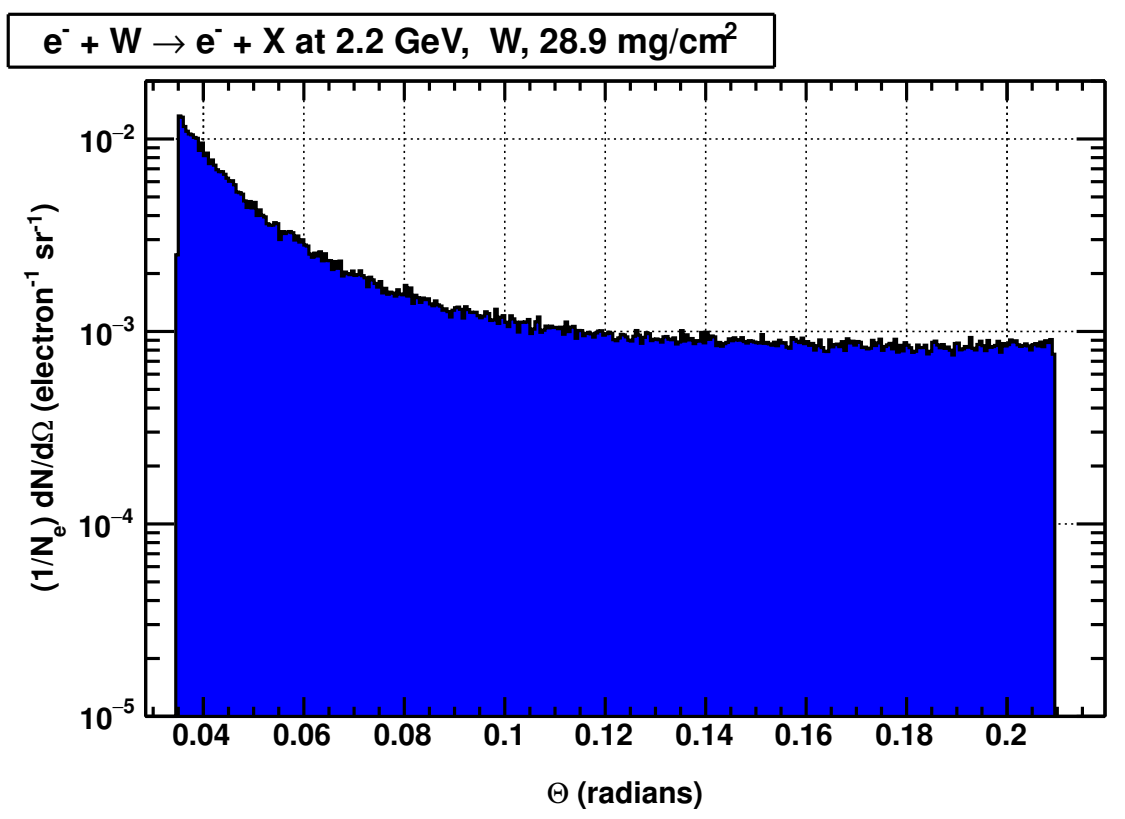

Figure 4.26: Electron production in a Tungsten target of thickness $28.9 \mathrm{mg} / \mathrm{cm}^{2}$ with an electron beam of $2.2 \mathrm{GeV}$ energy. $\mathrm{Y}$ axis is the number of events per solid angle per each electron incident on the target. $\mathrm{X}$ axis is the angle of electrons exiting the target with respect to the beam direction. 


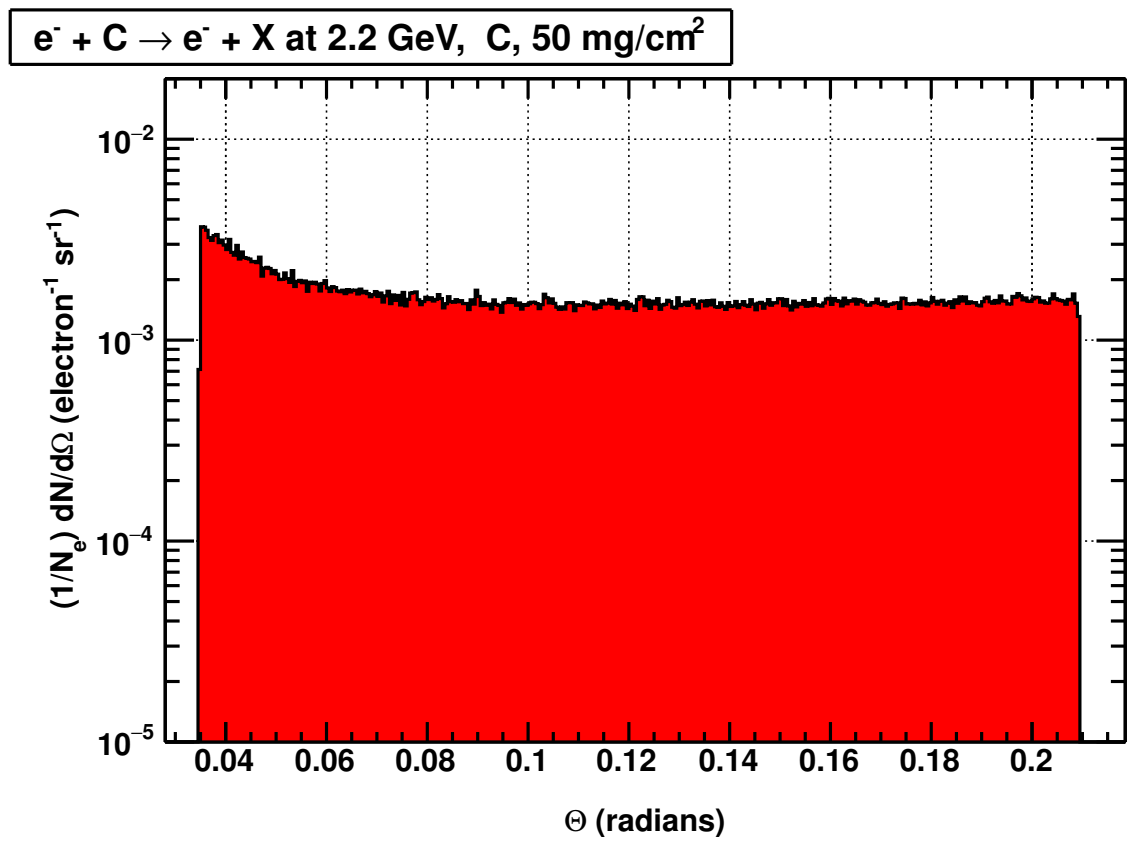

Figure 4.27: Electron production in a Carbon target of thickness $50 \mathrm{mg} / \mathrm{cm}^{2}$ with an electron beam of $2.2 \mathrm{GeV}$ energy. Y axis is the number of events per solid angle per each electron incident on the target. $\mathrm{X}$ axis is the angle of electrons exiting the target with respect to the beam direction. 
run can be given by,

$$
\text { Rate }_{\text {fiber }}=\frac{d N}{d \Omega} \times \frac{\phi_{\text {fiber }} L_{\text {fiber }}}{R^{2}} \times I_{\text {electron }}
$$

where $d N / d \Omega$ is the rate of particles at the SciFi detector location. $\phi_{f i b e r}(\sim 1 \mathrm{~mm})$ is the diameter of fiber and $L_{\text {fiber }}(\sim 10 \mathrm{~cm})$ is the length of fiber in the active area. $R(\sim 1 \mathrm{~m})$ is the distance from the target to the SciFi detector and $I_{\text {electrons }}$ is the beam current.

Equation 4.30 was used to calculate the expected single fiber rate of the SciFi detector during the proposed SciFi calibration run. From table 4.1, it is evident that the $e^{-}$rate is higher compared to the rates of $e^{+}$and $\gamma$. The value of $\phi_{\text {fiber }} L_{\text {fiber }}$ is $\sim 1 \mathrm{~cm}^{2}$ and the proposed beam current is $1 \mu \mathrm{A}$. These parameters gave an electron rate of $1200 \mathrm{kHz}$ at a $5^{\circ}$ angle. For each of these electron events, the SciFi will produce $\sim 4$ photo-electrons. Therefore, signal rate at PMT will be $\sim 5 \mathrm{MHz}$. The pulse pair resolution of maPMT Hamamatsu H7546A that will be used with the SciFi detector is several nano-seconds. Therefore, this rate is too high for the maPMT. For the optics calibration we need to either reduce the target thickness by two orders of magnitude or reduce the beam current in order to get optimal results.

Two Scintillating Fiber (SciFi) detectors will be used to calibrate the HRS optics in the JLab Hall A as a part of the APEX experiment. To evaluate the expected DAQ rates during the calibration runs, it was important to know how many photoelectrons will be produced in the PMT when an electron passed through the active area of the SciFi detector. We refer this quantity as the SciFi detector efficiency. One of the two SciFi detectors which will be used for APEX experiment, were tested at the Carnegie Mellon University and the second detector was tested at JLab. We calculated the SciFi efficiency and the expected DAQ rates during the calibration 
process. Results obtained from two independent experiments agreed and both SciFi detectors are ready to be commissioned before the APEX experiment. By using the SciFi detector to calibrate the HRS optics, we will be able to lower the uncertainty due to track measurement errors and finally achieve the required mass resolution of $0.5 \%$. 


\section{Chapter 5}

\section{Evaluation of neutron scattering}

\section{effects in a JLab radiation-detector}

\section{calibration facility using Bonner}

\section{spheres}

In order for the absolute comparisons described in Chapter 3 to be meaningful, it is critical to understand the JLab procedures for neutron-detector calibrations. The JLab Radiation Control Department (RadCon) was interested in performing a study to better understand room- and air-scattering effects during calibrations. Both to assist in helping this effort and to gain further confidence in the simulations presented here, we have collaborated with RadCon in this study and present our results. In addition to better defining the aforementioned scattering effects, we present simulations that predict absolute scattering rates with impressive accuracy.

The calibration facility at JLab is used to verify and calibrate personal dosimeters and area radiation monitors used at JLab. All calibration parameters need to be 
Chapter 5. Evaluation of neutron scattering effects in a JLab radiation-detector calibration facility using Bonner spheres

corrected to the presence of radiation fields in the calibration room, caused by the scattering of the radiation from the calibrated test sources in the floor and in the walls of the room. To calibrate neutron detectors, it is therefore important to characterize neutron scattering in the calibration room.

Table 5.1 summarizes features of a number of different types of neutron detectors [74]. Bonner sphere, labeled as Multisphere in the table, can measure neutrons in a wider energy range, but, with a lower resolution compared to other methods. In this chapter we discuss the measurements and simulations we performed using a set of Bonner spheres. We extracted the scatter-free counts rates in Bonner spheres measurements. We simulated the response curves of each Bonner sphere and predicted the scatter-free count rate. We developed a model of the calibration room and simulated the neutron scattering, which allowed us to do a direct comparison with measurements. In future work, the simulated Bonner sphere responses can be used to reconstruct scattered neutron spectra in the calibration room.

\subsection{Bonner sphere and its operation}

When neutrons traverse matter, they undergo elastic and/or inelastic scattering losing energy until they reach thermal energies, leave the moderator or are captured. The neutrons that reached thermal energies can be more easily captured by a thermal neutron detector. A novel neutron detector which included a spherical moderator that has found widespread use was first introduced in 1960 by Bramblett, Ewing, and Bonner [75], which subsequently became known as a Bonner sphere (BS). A Bonner sphere spectrometer (BSS) is a set of Bonner spheres with different moderator thicknesses. As mentioned in Table 5.1, thermal neutron detectors plus moderating sphere combinations (multisphere) can have a sensitivity to neutrons over a broad energy 
Chapter 5. Evaluation of neutron scattering effects in a JLab radiation-detector calibration facility using Bonner spheres

Table 5.1: Neutron spectrometer characteristics. Table was taken from Ref. [74].

\begin{tabular}{|c|c|c|c|c|}
\hline Spectrometer Type & $\begin{array}{l}\text { Energy } \\
\text { range } \\
(\mathrm{MeV})\end{array}$ & $\begin{array}{l}\text { Energy } \\
(\mathrm{MeV})\end{array}$ & $\begin{array}{l}\text { Resolu- } \\
\text { tion } \\
\text { (FWHM) }\end{array}$ & $\begin{array}{l}\text { Detec- } \\
\text { tion } \\
\text { efficiency }\end{array}$ \\
\hline Recoil proportional counter & $0.05-5$ & 1 & $10 \%^{\mathrm{a}}$ & $3 \%$ \\
\hline Organic scintillator & $2-150$ & 8 & $4 \%^{\mathrm{a}}$ & $20 \%$ \\
\hline Recoil proton telescope & $1-250$ & 60 & $4 \%^{\mathrm{a}}$ & $<0.05 \%$ \\
\hline Capture-gated & $1-20$ & 5 & $50 \%{ }^{\mathrm{a}}$ & $1 \%$ \\
\hline $\begin{array}{l}{ }^{3} \text { He gridded ionization } \\
\text { chamber }\end{array}$ & $0.05-10$ & 1 & $2 \% \mathrm{a}$ & $0.3 \%$ \\
\hline $\begin{array}{l}{ }^{3} \text { He-semiconductor } \\
\text { sandwitch }\end{array}$ & $0.1-20$ & 1 & $50 \mathrm{keV}^{\mathrm{a}}$ & $0.1 \%$ \\
\hline Diamond semiconductor & $8-20$ & 14 & $1 \% \mathrm{~b}$ & $1 \%$ \\
\hline Time-of-flight & $1-15$ & 2.5 & $5 \% \mathrm{c}$ & $0.05 \mathrm{~cm}^{-2}$ \\
\hline Foil radioactivation & $0.2-20$ & - & - & - \\
\hline Superheated drop (bubble) & $0.1-20$ & - & - & - \\
\hline Multisphere & $10^{-8}-200$ & - & - & - \\
\hline
\end{tabular}

\footnotetext{
${ }^{\text {a }}$ Pulse height resolution

${ }^{\mathrm{b}}$ Energy resolution

c Time-of-flight resolution
} 
Chapter 5. Evaluation of neutron scattering effects in a JLab radiation-detector calibration facility using Bonner spheres

range. Hydrogen $\left({ }^{1} \mathrm{H}\right)$ is considered as a good moderator for neutrons as the proton mass is quite close to that of the neutron, resulting neutrons losing almost all of their energy in a single head-on collision. But, ${ }^{2} \mathrm{H}$, Be and $\mathrm{C}$ are also considered to be good moderators. In a BSS the moderating material is polyethylene which contains $14 \%$ of ${ }^{1} \mathrm{H}$ and $86 \%$ of $\mathrm{C}$. The moderator being spherical provides an isotropic response to neutrons. As mentioned previously, a BS is a polyethylene sphere with a thermal neutron detector at the center of the sphere. Spheres with smaller diameter offer less moderation and mostly low energy neutrons are captured by the thermal detector. Larger spheres provide more moderation, increasing the probability of high energy neutrons being detected.

The very first BS developed by Bramblett, Ewing and Bonner used a 4 mm thick, $4 \mathrm{~mm}$ diameter cylindrical Europium Doped Lithium Iodide $\left(\mathrm{Li}^{6} \mathrm{I}(\mathrm{Eu})\right)$ crystal positioned at the center of the polyethylene sphere as the thermal neutron detector. The set of spheres consisted of five spheres of diameters ranging between 2- and 12-inch. Any detector can be characterized by a response curve, which is given in counts per unit fluence (counts/n/cm2). The response function of these BS's have been experimentally determined and it is shown in Figure 5.1. A response function is the It is clear that the peak of the response functions moves to higher energies as the sphere size increases. If a sphere $i$ has response function $R_{i}(E)$, and is in a neutron field with a spectral fluence $\phi(E)$, then the measured neutron counts $M_{i}$ from the $i^{\text {th }}$ sphere can be expressed by a convolution of $R_{i}(E)$ and $\phi(E)$ :

$$
M_{i}=\int R_{i}(E) \phi(E) d E
$$

A more practical equation turns out to be in the form of discrete energy ranges. 
Chapter 5. Evaluation of neutron scattering effects in a JLab radiation-detector

$$
M_{i}=\sum_{j=1}^{n} R_{i j} \phi_{j}
$$

where $\phi_{j}$ is the fluence in group $j$ extending from energy $E_{j}$ to $E_{j+1}$ and $R_{i j}$ represents $R_{i}(E)$ averaged over group $j$.

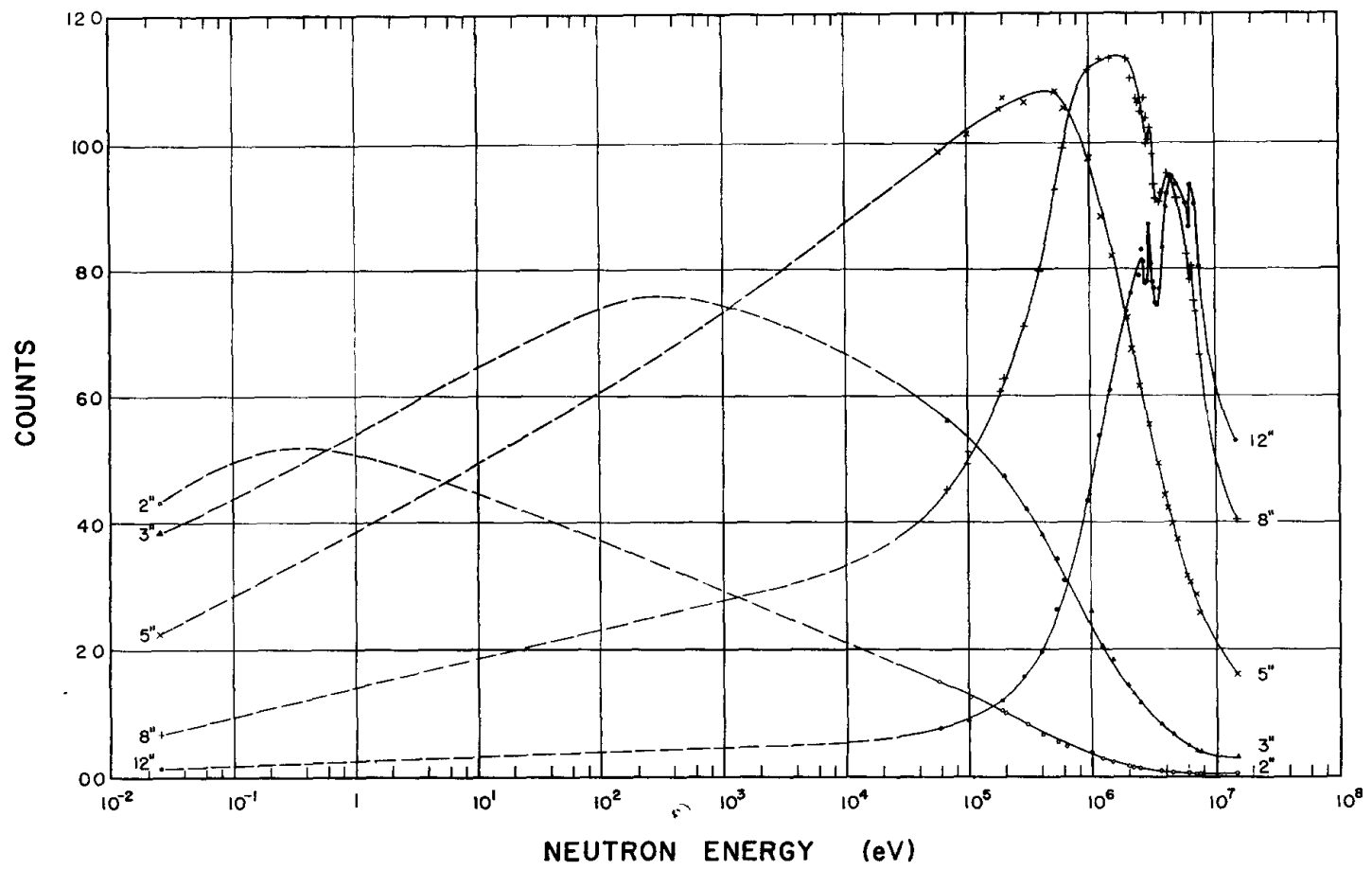

Figure 5.1: Response functions of original Bonner sphere set. These are counts per $10^{6}$ neutrons emitted from an isotropic point source $40 \mathrm{~cm}$ away. This figure was taken from the Ref. [75]

The solution, $\phi_{j}$, of the equation 5.1, also known as the Fredholm integral equation is undetermined and complex. If we use a number of spheres $m$ to measure an unknown neutron field, then the equation 5.2 represents a set of $m$ linear equations. If $m \geq n$ then they can be easily solved and values of $\phi_{j}$ can be obtained. But, usually number of spheres $m$ are less than 10 , so that spectrum is represented by 
Chapter 5. Evaluation of neutron scattering effects in a JLab radiation-detector calibration facility using Bonner spheres

an array with $n \geq m$. These can only be solved either by trial-and-error or with additional priori information about the spectra. Many methods and algorithms have been proposed to solve this so-called inverse problem [76][77].

Since the very first Bonner sphere, people have been trying different alternatives for the thermal neutron detector. One reason was that with increasing fluence of

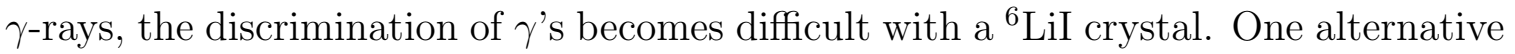
is to use a ${ }^{3} \mathrm{He}$ proportional counter. As illustrated in Figure 5.2 [78] the neutron capture cross section of ${ }^{3} \mathrm{He}$ follows roughly $1 / \sqrt{\mathrm{E}}$ behavior, $\mathrm{E}$ being the incident neutron energy. The nuclear reaction that takes place in ${ }^{3} \mathrm{He}$ is:

$$
{ }^{3} \mathrm{He}+\mathrm{n} \longrightarrow{ }^{3} \mathrm{H}+{ }^{1} \mathrm{H}+765 \mathrm{keV}
$$

This reaction is exothermic and releases energetic charged particles into the gas. The neutron causes the breakup of the nucleus into a tritium nucleus, ${ }^{3} \mathrm{H}$, and a proton, ${ }^{1} \mathrm{H}$. The triton and the proton share the $765 \mathrm{keV}$ reaction energy. The cross section for this reaction is about $5330 \mathrm{~b}$ for thermal neutrons. Figure 5.3 illustrates a typical pulse-height spectrum from a ${ }^{3} \mathrm{He}$ proportional counter [78]. The full energy peak at $765 \mathrm{keV}$ in the spectrum represents the sum of the kinetic energy of both the triton and the proton. If one or the other particle leaves the ${ }^{3} \mathrm{He}$ detector, less energy is collected in the gas, which results in a low energy tail. The increase at low energy is due to noise and piled up $\gamma$-ray events.

Many improvements have been made over the years to make measurements more accurate when using BSS's. Typical Bonner spheres with polyethylene moderators have very low response above $20 \mathrm{MeV}$. The BSS can be modified to measure high energy neutrons around high-energy accelerators by adding lead, iron or copper layers within or outside the polyethylene spheres [79][80][81]. Even though a BSS gives very 
Chapter 5. Evaluation of neutron scattering effects in a JLab radiation-detector calibration facility using Bonner spheres

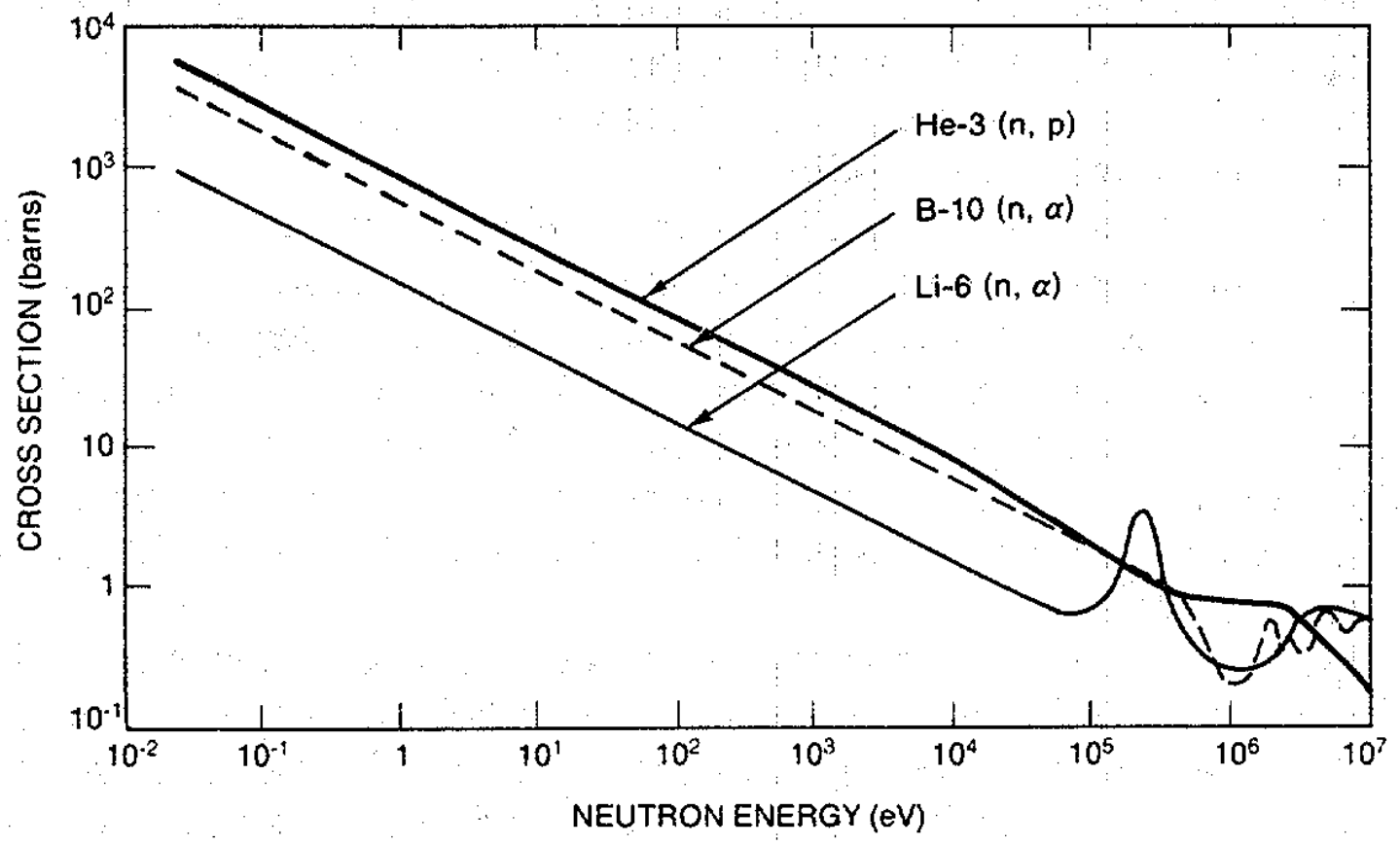

Figure 5.2: ${ }^{3} \mathrm{He}(\mathrm{n}, \mathrm{p}),{ }^{10} \mathrm{~B}(\mathrm{n}, \alpha)$ and ${ }^{6} \mathrm{Li}(\mathrm{n}, \alpha)$ cross sections as a function of incident neutron energy [78]. 
Chapter 5. Evaluation of neutron scattering effects in a JLab radiation-detector calibration facility using Bonner spheres

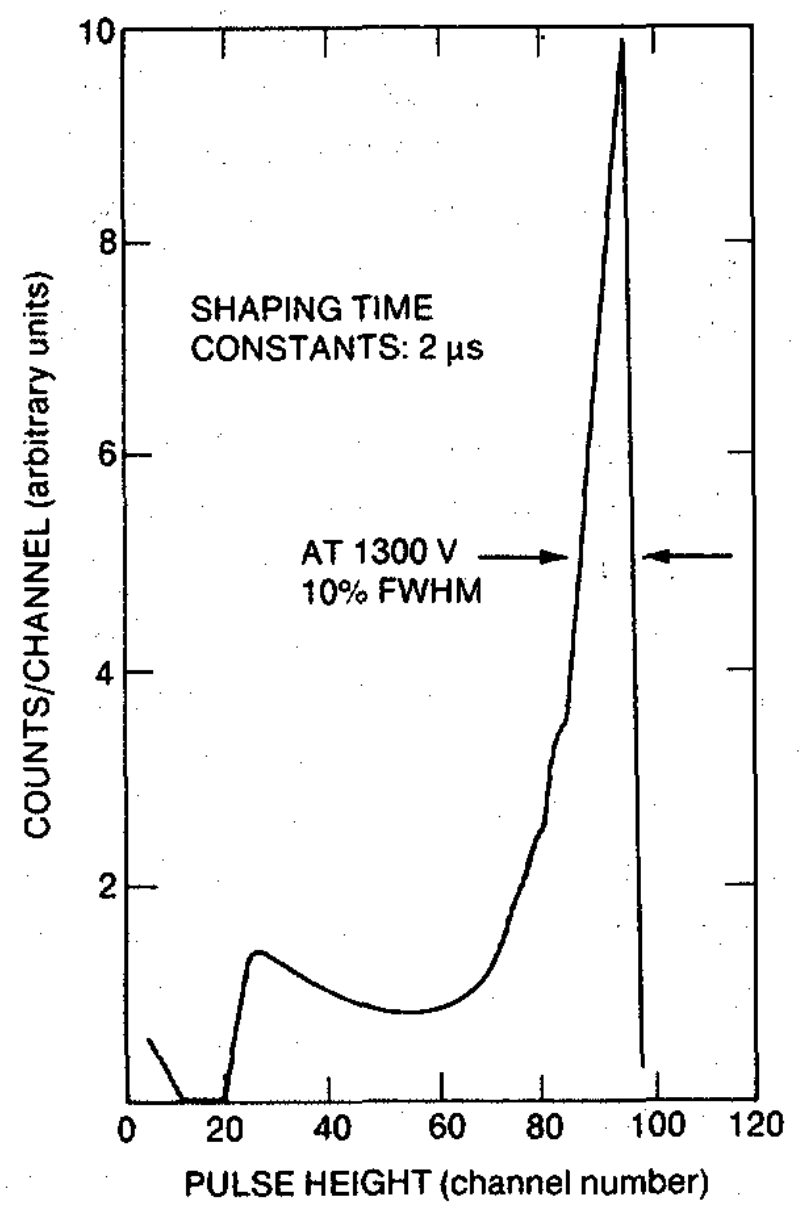

Figure 5.3: A typical pulse-height spectrum for a thermal neutrons detected by a ${ }^{3}$ He filled counter [78]. 
Chapter 5. Evaluation of neutron scattering effects in a JLab radiation-detector calibration facility using Bonner spheres

good energy response, operating Bonner spheres is a bit difficult due to their size and the number of separate detectors. A new type of neutron spectrometer has been developed based on the principle of a BSS [82]. In this detector the moderators are cylindrical in shape compared to spherical moderators in a BSS. The different thicknesses of moderators are formed by inserting one cylinder into another, like nested Russian dolls. This nested neutron spectrometer is available commercially. Another important improvement is the reduction of the effort which goes into the calculation of the response functions. With modern fast computers and monte-carlo simulation techniques, the response functions can be calculated more accurately and efficiently. Figure 5.4 shows response functions calculated using GEANT4 montecarlo package [83].

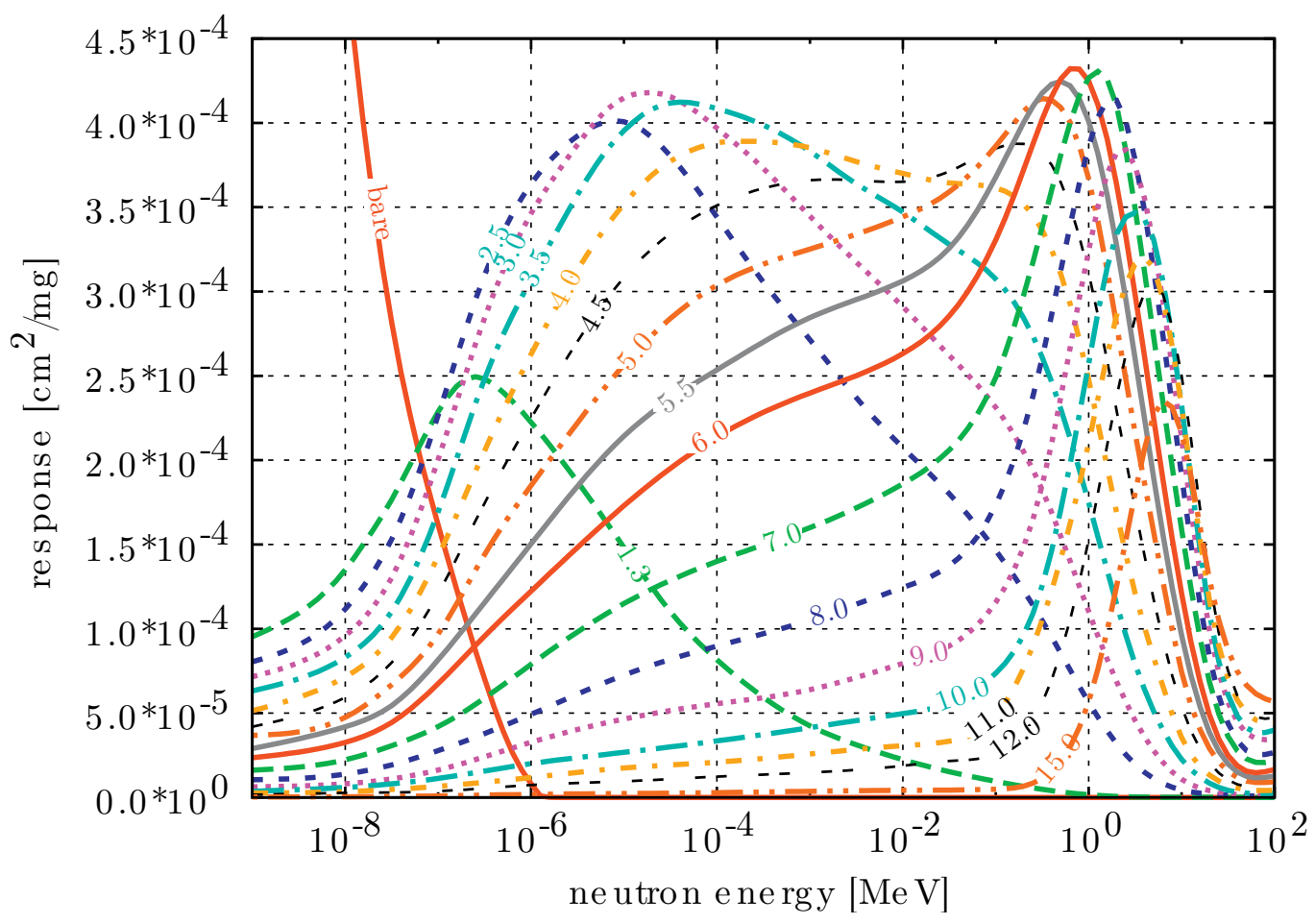

Figure 5.4: Response functions of Bonner sphere spectrometer set calculated with GEANT4. Gold foil is used inside of spheres as the thermal neutron detector. Sphere size in inches is indicated on each line [83]. 
Chapter 5. Evaluation of neutron scattering effects in a JLab radiation-detector calibration facility using Bonner spheres

\subsection{A Bonner sphere Spectrometer at JLab}

The JLab Radiation Control Department (RadCon) has constructed a BSS which consists of 5 BS's. These are polyethylene spheres of 5 -inch $(12.7 \mathrm{~cm}), 8$-inch $(20.32 \mathrm{~cm})$, 10-inch $(25.4 \mathrm{~cm}), 12$-inch $(30.48 \mathrm{~cm})$ and an extended range Bonner sphere of 10-inch $(25.4 \mathrm{~cm})$ which includes 0.7 -inch $(1.78 \mathrm{~cm})$ thick lead. Figure 5.5 illustrates a dia-

gram of a cross-section of the extended range BS. In this extended range BS, a shell of polyethylene around the ${ }^{3} \mathrm{He}$ counter is removed and replaced with a 0.7 -inch thick layer of lead to make the detector more sensitive to high energy neutrons.
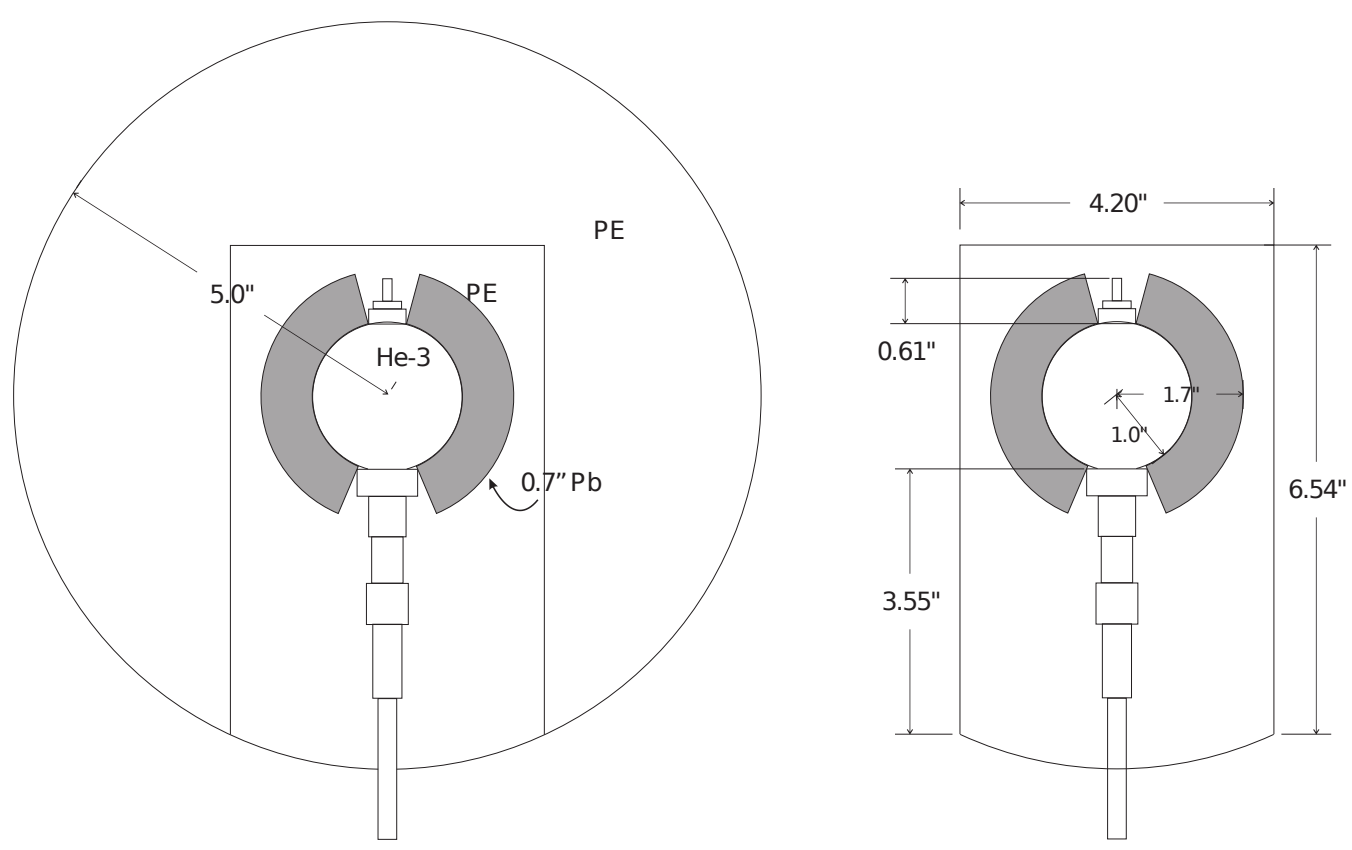

Figure 5.5: A cross-sectional side view of one of the Bonner spheres. The diameter of the sphere is 10 inches and there is a 0.7 -inch thick lead layer inside of the sphere. The spherical proportional counter is filled with $10 \mathrm{~atm}{ }^{3} \mathrm{He}$ gas.

These five moderating spheres at JLab are made out of high-density polyethylene (VERSADUR High-Density Polyethylene - 600 Series) of density $0.963 \mathrm{~g} / \mathrm{cm}^{3}$. The 
Chapter 5. Evaluation of neutron scattering effects in a JLab radiation-detector calibration facility using Bonner spheres

thermal neutron detector is a ${ }^{3}$ He proportional counter Model LND-2705 manufactured by LND Inc. It is a 2-inch diameter spherical counter filled with 10 atm of ${ }^{3} \mathrm{He}$ gas. The spherical construction ensures a more uniform response to radiation from every direction. The center wire acts as the anode and the 0.02-inch thick stainlesssteel spherical wall acts as the cathode of the detector. The operating voltage range of the proportional counter is $1450-1700 \mathrm{~V}$ and it is connected to an amplifier module with a SHV connector. Figure 5.6 shows all 5 Bonner spheres and the bare detector. Each BS has its own ${ }^{3} \mathrm{He}$ counter and an amplifier module.

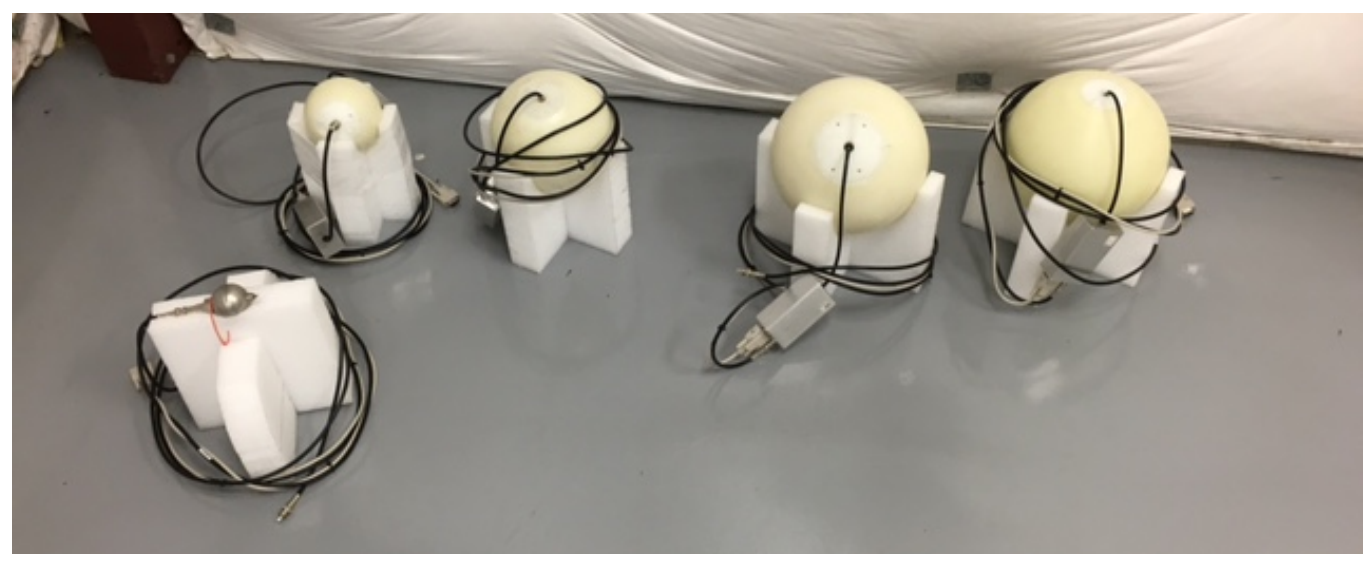

Figure 5.6: The Bonner sphere spectrometer at Jefferson Lab contains 5 separate spheres. The diameters of the spheres are 5-inch, 8-inch, 10-inch and 12-inch. There is an extended Bonner sphere of 10-inch diameter with a $\mathrm{Pb}$ layer.

Figure 5.7 provides a block diagram of the DAQ system used with each Bonner sphere. Front-end electronics such as a charge sensitive amplifier (CSA), a pulse shaper and a linear amplifier are housed in an aluminum enclosure which I earlier referred to as an amplifier module. Details of the front-end electronics can be found in a JLab technical note [88]. This signal was fed into a high-quality PCI audio card ("Audiophile-192" by M-Audio) in the DAQ computer workstation for continuous readout. Each audio card functioned as a two channel, 24-bit/192 kHz sampling rate ADC of which the 
Chapter 5. Evaluation of neutron scattering effects in a JLab radiation-detector calibration facility using Bonner spheres

frequency response is between $20 \mathrm{~Hz}-80 \mathrm{kHz}$. All six proportional counters were powered from a single interface and power module. The DAQ control, readout and analysis software [87] provided continuous detection of the neutron capture signals in the input data streams, determining signal parameters and storing them into a raw event database. Based on these information, neutron capture rates in each detector were calculated.

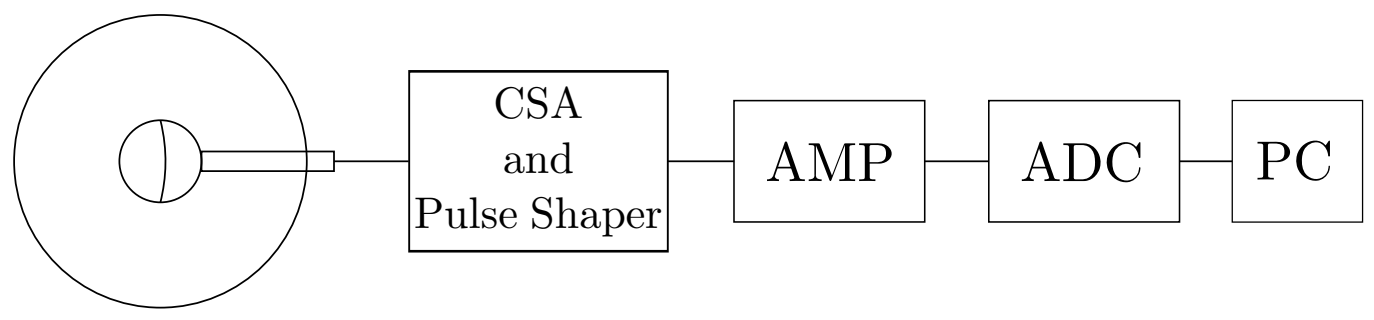

Figure 5.7: Block diagram of the experimental setup.

\subsection{Calibration of the BSS with an ${ }^{241} \mathrm{Am}-\mathrm{Be}$ source}

There are several standard calibration techniques to do scatter corrections and evaluate scatter-free response of the detectors. The shadow-cone method involves the construction of a cone using iron and polyethylene. This cone is placed between the source and the BS. The direct neutrons from the source will be blocked by this cone and only scattered neutrons are counted by the detector. Shadow-cone technique requires construction of different sized cones for each Bonner sphere. A second calibration technique which does not involve building mechanical shields is called "polynomial fit model" [84]. In this method, measured count rates are fitted with a polynomial and the scattering contributions are calculated from the fit parameters. The application of this method for scatter correction will be discussed later in this chapter. 
Chapter 5. Evaluation of neutron scattering effects in a JLab radiation-detector calibration facility using Bonner spheres

\subsubsection{Calibration facility at JLab}

The JLab Radiation Control Department maintains an on-sight detector calibration facility where they calibrate area monitoring devices and personal dosimeters. Figure 5.8 illustrates a dimensions of the calibration room.

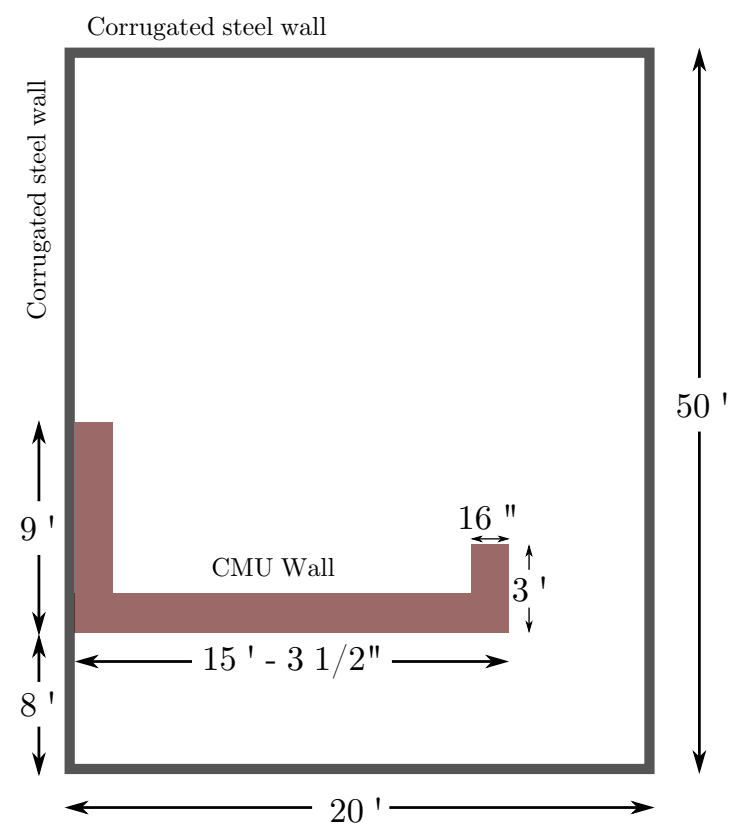

Top View

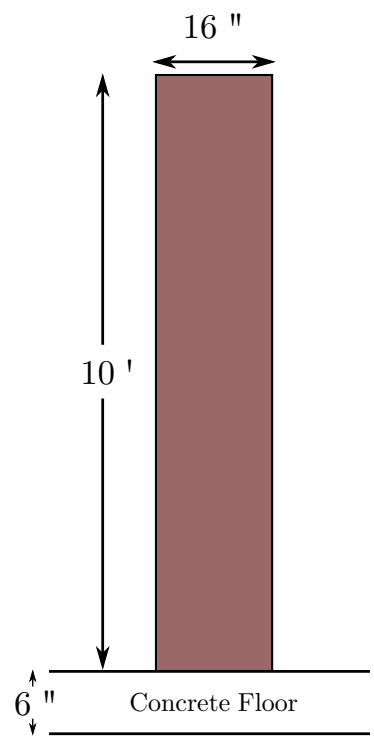

Elevated View

Figure 5.8: Dimensions of the detector calibration room at Jefferson Lab. The drawing on the left shows the top view of the room and the drawing on the right shows a side view of the back wall constructed of concrete masonry unit (CMU). The height of the room is $12 \mathrm{ft}$ and the height of the CMU wall is $10 \mathrm{ft}$.

The calibration room dimensions are $50 \times 20 \mathrm{ft}$ with a 12 foot height. The room's walls and ceiling are constructed of steel. The walls of the calibration room are 24 gauge corrugated steel and there is an insulation layer inside. The roof is 22 gauge corrugated steel and the room has a 6-inch thick concrete floor. A new addition to the room is a 16-inch thick concrete wall made from concrete masonry units (CMU wall). This wall separates the room operator from the radiation environment. 
Chapter 5. Evaluation of neutron scattering effects in a JLab radiation-detector calibration facility using Bonner spheres

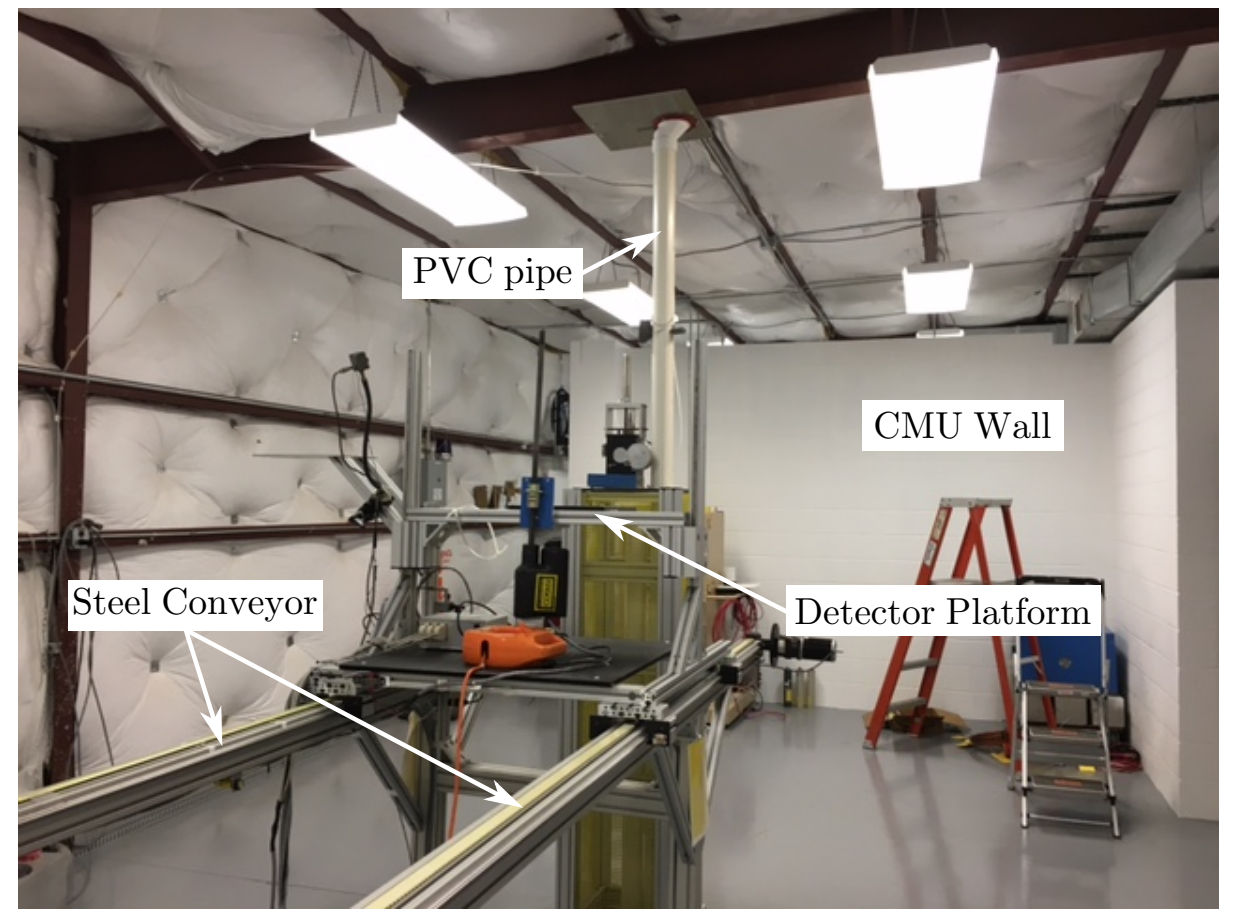

Figure 5.9: A photograph of the calibration room at JLab. The wall constructed of concrete masonry units (CMU) is visible and the controls of the steel conveyor and the neutron source is located behind the CMU wall. Neutron source is located below the ground and moves within the PVC pipe shown in the photograph. 
Chapter 5. Evaluation of neutron scattering effects in a JLab radiation-detector calibration facility using Bonner spheres

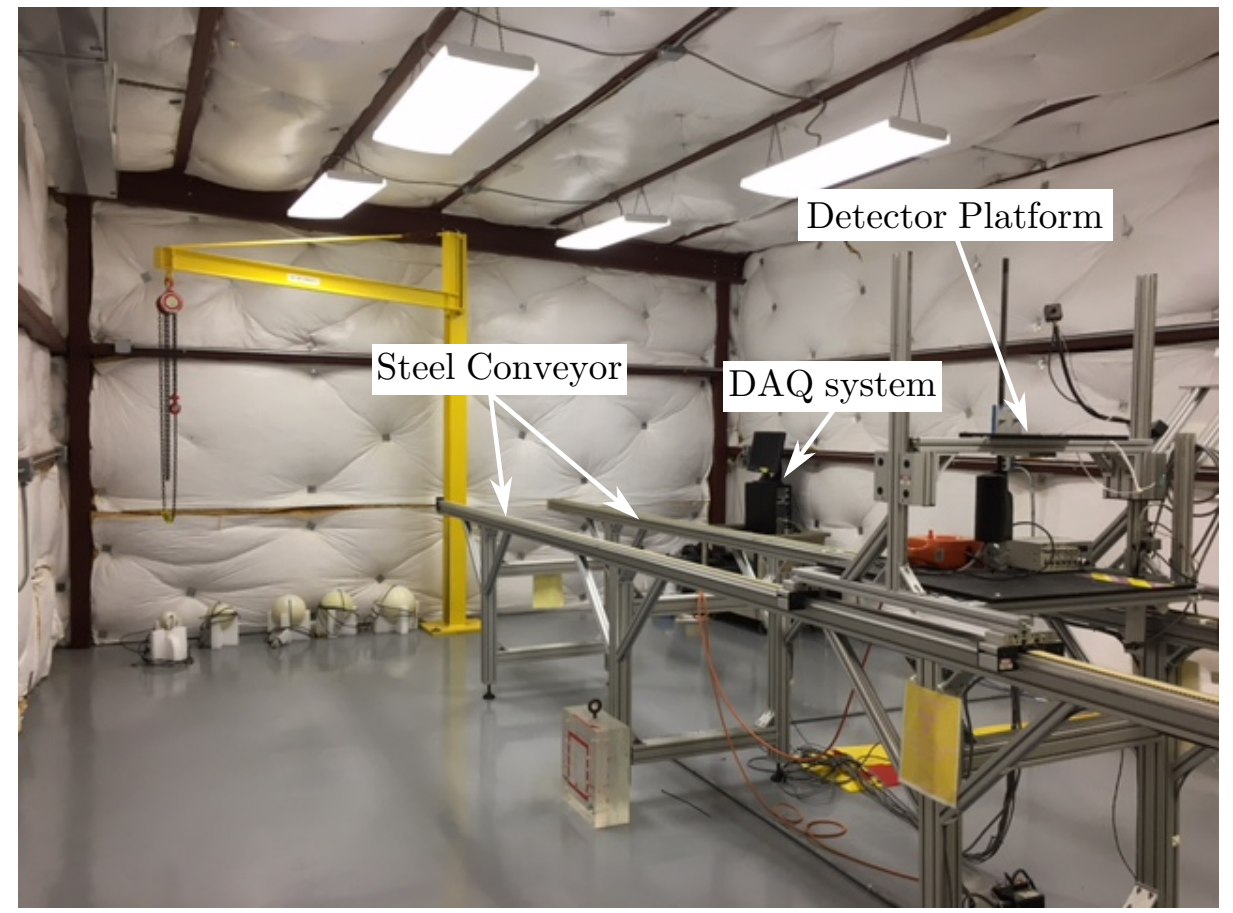

Figure 5.10: A photograph of the calibration room at JLab. The detector platform can move along the steel conveyor, up/down or left/right. The maximum source-todetector distance is $\sim 5 \mathrm{~m}$. 
Chapter 5. Evaluation of neutron scattering effects in a JLab radiation-detector calibration facility using Bonner spheres

A NIST calibrated Am-Be neutron source is available for calibration purposes. This source has activity of $5 \mathrm{Ci}$ and a neutron emission rate of $1.094 \times 10^{7} \pm 2.9 \%$ neutrons per second. The source is secured in a cylindrical stainless steel container and located below the ground level. Figure 5.9 and Figure 5.10 illustrate two views of the calibration room. The source is moved above and below the ground level within a PVC pipe indicated in Figure 5.9 using a mechanical pulley system. When the source is in use it is located $\sim 90$ inches above ground and $\sim 15$ feet from the $\mathrm{CMU}$ wall. The detector platform has three degrees of freedom. As is visible in Figures 5.9 and 5.10, the detector platform can be moved away or towards the neutron source on a steel conveyor with two parallel rails. The platform can move along the conveyor for about $5 \mathrm{~m}$, across the conveyor for a very limited range and up/down again for a very limited range. This allowed us to carefully align the source and the detector. Detector platform motion controllers were located behind the CMU wall.

\subsubsection{Evaluate scatter-free count rates in Bonner spheres}

The Bonner sphere spectrometer needs to be calibrated based solely on the neutrons coming directly from the neutron source. Therefore, the detector counts needs to be corrected for scattering effects from walls, floor, ceiling and air. The following experiment was performed to evaluate these effects. Bonner sphere measurements were carried out at 9 different separation distances, from the source center to the center of the detector, ranging from $100 \mathrm{~cm}$ to $500 \mathrm{~cm}$. Figure 5.11 shows the experimental setup in which a 12-inch sphere was mounted on the detector platform and aligned with the source center. The data were acquired for several minutes until we obtained reasonable statistics. Figure 5.12 (left) shows pulse height spectra generated from different diameter Bonner spheres for a source-to-detector distance of $1 \mathrm{~m}$. Figure 
Chapter 5. Evaluation of neutron scattering effects in a JLab radiation-detector calibration facility using Bonner spheres

5.12 (right) shows neutron event rates calculated based on these pulse height spectra. As illustrated in Figure 5.13, the neutron count rates were plotted against the source-to-detector distance for all 6 Bonner spheres.

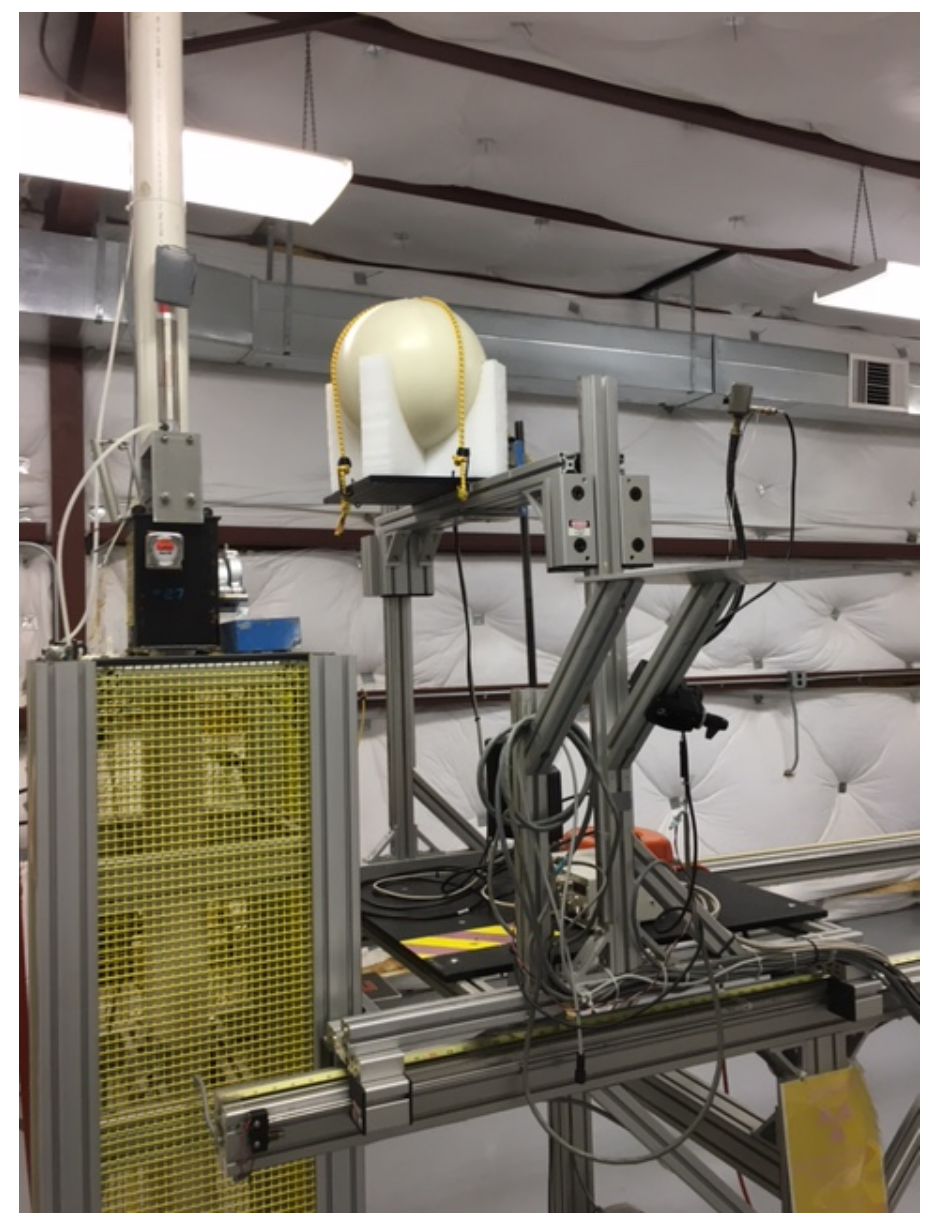

Figure 5.11: Experimental setup for BSS calibration. 12-inch sphere is mounted on the platform.

As mentioned at the beginning of this section, we used the polynomial fit model proposed by J.B. Hunt to evaluate scattering contributions (Ref. [84]), which can be described by, 
Chapter 5. Evaluation of neutron scattering effects in a JLab radiation-detector calibration facility using Bonner spheres

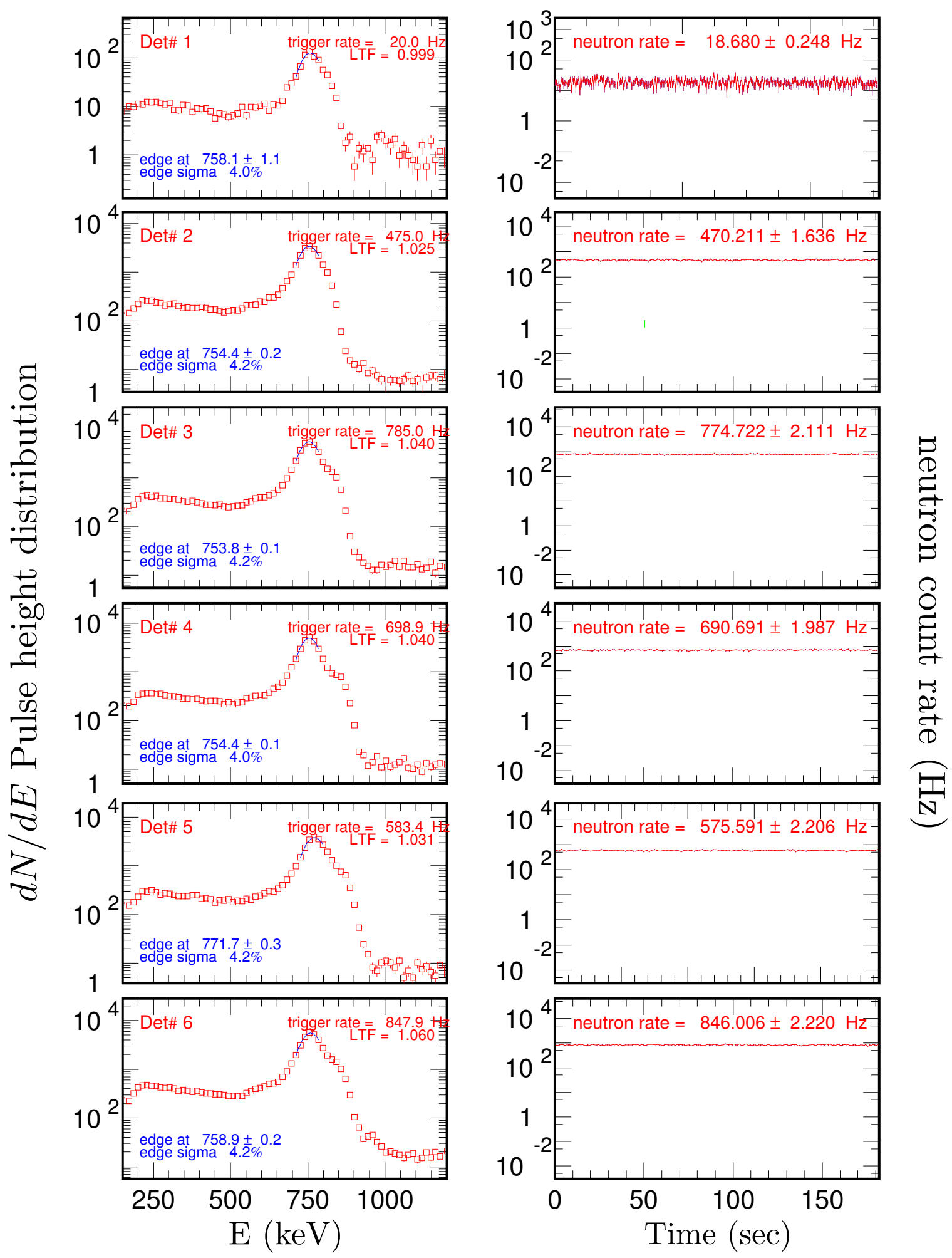

Figure 5.12: (left) Pulse height spectra from all the Bonner spheres located $1 \mathrm{~m}$ from the Am-Be neutron source. (right) Neutron rate for the corresponding sphere in $\mathrm{Hz}$. 
Chapter 5. Evaluation of neutron scattering effects in a JLab radiation-detector

$$
C(d)=K\left(\frac{1}{d^{2}}+\frac{A}{d}+R\right)
$$

where $C$ is the count rate, $K$ is the scatter free count rate, $A$ represents the factor due to the air scattering, $R$ is the factor due to scattering from other elements in the room including walls, roof and the floor and the $d$ is the distance from the center of the source to the center of the detector. A geometric correction factor was not included in the equation as the geometric correction factor is unity at distances larger than $1 \mathrm{~m}$.

Radiation from a point source should follow the inverse square law. Any deviation from this behavior should be due to scattering effects. In the polynomial model, shown in equation 5.4, for an isotropic emitting source, the contribution to the detector counts due to scattering by the walls is assumed to be a constant. The contribution due to scattering in air and by the supporting structures is assumed to be inversely proportional to the distance $d$. Multiplying both sides of the equation by $d^{2}$ gives

$$
C(d) d^{2}=K\left(1+A d+R d^{2}\right)
$$

According to Hunt, the air scattering factor A and the room scattering factor $\mathrm{R}$ can be obtained by fitting the data with the polynomial distribution shown in equation 5.5. Figure 5.13 shows 6 different plots, each corresponds to data obtained from a Bonner sphere. As shown in Figure 5.13, we used the equation 5.5 to fit count rate vs. distance measurements for all 6 Bonner spheres.

Table 5.2 summarizes the best fit parameters for polynomial fits. The fitting parameter $\mathrm{R}$ is smaller compared to the parameter $\mathrm{A}$ and for some spheres the value of $\mathrm{R}$ is negative. A negative $\mathrm{R}$ value has no physical meaning and typically the 
Chapter 5. Evaluation of neutron scattering effects in a JLab radiation-detector calibration facility using Bonner spheres

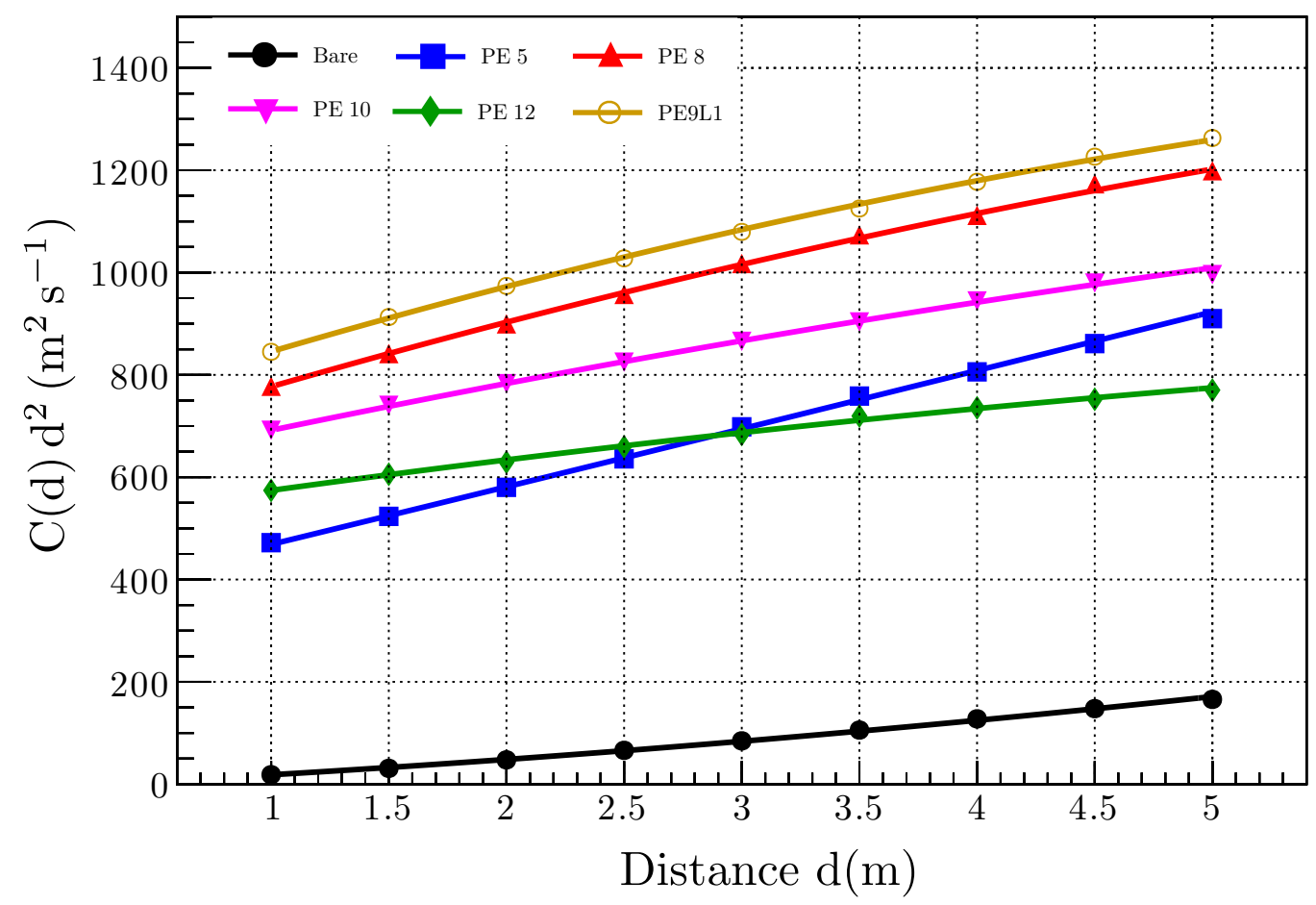

Figure 5.13: Measured count rates using Bonner spheres are multiplied by $d^{2}$ and plotted as a function of source-to-detector distance $d$. The data were fitted using a second order polynomial. Best fit parameters are indicated in Table 5.2.

Table 5.2: Best fit parameters of second order polynomial fits for BSS count rates as a function of source-to-detector distance.

\begin{tabular}{ccccc}
\hline \multirow{2}{*}{$\begin{array}{c}\text { Bonner } \\
\text { sphere }\end{array}$} & \multicolumn{4}{c}{ Polynomial fit parameters $\mathrm{K}\left(1+\mathrm{Ad}+\mathrm{Rd}^{2}\right)$} \\
\cline { 2 - 5 } & $\mathrm{K}(\mathrm{Hz})$ & $\mathrm{A}$ & $\mathrm{R}$ & $\chi^{2} / \mathrm{ndf}$ \\
\hline PE 5 & $356.8 \pm 3.0$ & $0.313 \pm 0.008$ & $+1.00 \times 10^{-3}$ & $34.600 / 6$ \\
PE 8 & $638.0 \pm 3.6$ & $0.228 \pm 0.005$ & $-1.00 \times 10^{-2}$ & $16.190 / 6$ \\
PE 10 & $592.1 \pm 5.1$ & $0.175 \pm 0.008$ & $-6.83 \times 10^{-3}$ & $3.656 / 6$ \\
PE 12 & $508.2 \pm 3.5$ & $0.136 \pm 0.007$ & $-6.22 \times 10^{-3}$ & $6.517 / 6$ \\
PE9L1 & $702.9 \pm 4.1$ & $0.214 \pm 0.006$ & $-1.00 \times 10^{-2}$ & $5.283 / 6$ \\
\hline
\end{tabular}


Chapter 5. Evaluation of neutron scattering effects in a JLab radiation-detector calibration facility using Bonner spheres

contribution from the room scatter is larger than the air scatter.

If the calibration facility has walls (including ceiling and floor) all made of concrete (a bunker-room), then the neutrons scatter around until they get absorbed without leaving the room. In this way we can imagine there is a constant neutron field in the room due to scattering from walls. The measurements taken by Hunt and colleagues were done in a bunker-room. But, the calibration room at JLab has very thin steel walls. Therefore, most of the neutrons leave the building. We believe most of the neutrons scattering occurs in the JLab calibration room due to the concrete floor and the soil and that it is not well described by the constant term, $R$, in equation 5.5. Therefore, the polynomial method alone should not be used to separate scattering contribution from room and air. Hence, before polynomial method can be used in the JLab calibration room for routine detector calibrations, it must be carefully checked against another method, such as the shadow cone method.

We suggest that a linear model is sufficient to describe the neutron scattering in the calibration room at JLab. Therefor we used a linear model,

$$
C(d) d^{2}=p_{0}+p_{1} d
$$

where $p_{0}$ is the un-scattered neutron contribution and $p_{1}$ is the scattered contribution, to fit the distribution of count rate multiplied by the square of the distance as a function of distance. Best fit parameters for $p_{0}$ and $p_{1}$ is given in Table 5.3.

\subsubsection{Evaluate scatter-free count rates using simulations}

We can calculate scatter-free count rates in BS's using the following equation 
Chapter 5. Evaluation of neutron scattering effects in a JLab radiation-detector calibration facility using Bonner spheres

Table 5.3: Best fit parameters of linear fits for BSS count rates as a function of source-to-detector distance.

\begin{tabular}{cccc}
\hline \multirow{2}{*}{$\begin{array}{c}\text { Bonner } \\
\text { sphere }\end{array}$} & \multicolumn{3}{c}{ Linear fit parameters $\mathrm{p}_{0}+\mathrm{p}_{1} \mathrm{~d}$} \\
\cline { 2 - 4 } & $p_{0}(\mathrm{~Hz})$ & $p_{1}(\mathrm{~Hz})$ & $\chi^{2} / \mathrm{ndf}$ \\
\hline PE 5 & $355.1 \pm 1.1$ & $113.2 \pm 0.6$ & $34.98 / 7$ \\
PE 8 & $676.1 \pm 1.5$ & $108.1 \pm 0.6$ & $155.5 / 7$ \\
PE 10 & $612.7 \pm 2.2$ & $82.1 \pm 0.9$ & $23.54 / 7$ \\
PE 12 & $520.7 \pm 1.4$ & $54.4 \pm 0.9$ & $21.47 / 7$ \\
PE9L1 & $737.2 \pm 1.6$ & $111.5 \pm 1.0$ & $88.01 / 7$ \\
\hline
\end{tabular}

$$
C_{i}=\Phi_{\text {ref }} \sum_{j=1}^{60} R_{i j} \phi_{j}^{A m-B e}
$$

where $\Phi_{\text {ref }}$ is the scatter-free neutron fluence, $R_{i j}$ is the response functions of a BS and $\phi_{j}^{A m-B e}$ is the normalized standard Am-Be spectrum. The value $\Phi_{\text {ref }}$ can be calculated by using following equation

$$
\Phi_{r e f}=\frac{B}{4 \pi d^{2}} e^{-\Sigma\left(d-r_{i}\right)}
$$

where $B$ is the source strength, $d$ is the distance from the source, $r_{i}$ is the sphere radius and $\Sigma$ is the air attenuation coefficient which is $890 \times 10^{-7} \mathrm{~cm}^{-1}$ [85] for an Am/Be source. Since the variation of the quantity $e^{-\Sigma\left(d-r_{i}\right)}$ with the sphere diameter is negligible, the same $\Phi_{\text {ref }}$ was considered for all the spheres. For $100 \mathrm{~cm}$ source-todetector distance and with $B=1.1 \times 10^{7} \mathrm{~s}^{-1}$ source strength, the $\Phi_{\text {ref }}$ is $86.4 \mathrm{~cm}^{-2} \mathrm{~s}^{-1}$. Figure 5.15 illustrates the IAEA Am-Be spectrum [49] used for all the calculations in this study. Geant4 simulations were used to calculate the response curves of all BS's and the details of the calculations will be discussed in section 5.5. Figure 5.21 shows 
Chapter 5. Evaluation of neutron scattering effects in a JLab radiation-detector calibration facility using Bonner spheres

the calculated BS response curves.

Table 5.4 summarizes scatter-free count rates calculated using the data from Table 5.3 (the parameter $p_{0}$ ). The third column of the table presents the absolute count rates in the absence of wall- and air-scattering predicted using Geant4 and the known properties of the Am/Be source using equation 5.7. The calculation of Bonner sphere response functions went into equation 5.7 are present in section 5.5. The ratios of measurements to simulations are close to one which indicates an excellent agreement with a root-mean-square-error of 0.09 .

Table 5.4: A comparison of scatter-corrected count rates and absolute count rates is given. The scatter corrected count rates were calculated based on Bonner sphere measurments. The absolute count rates were calculated based on simulated Bonner sphere response and the known properties of the calibrated Am/Be source.

\begin{tabular}{cccc}
\hline $\begin{array}{c}\text { Bonner } \\
\text { sphere }\end{array}$ & $\begin{array}{c}\text { Corrected } \\
\text { count rate } \\
(\mathbf{H z})\end{array}$ & $\begin{array}{c}\text { Absolute } \\
\text { count rate } \\
(\mathbf{H z})\end{array}$ & $\begin{array}{c}\text { Ratio } \\
\text { corrected/absolute }\end{array}$ \\
\hline PE 5 & 355.1 & 364.0 & 0.98 \\
PE 8 & 676.1 & 672.1 & 1.01 \\
PE 10 & 612.7 & 620.8 & 0.99 \\
PE 12 & 520.7 & 497.6 & 1.05 \\
PE9L1 & 737.2 & 916.6 & 0.80 \\
\hline
\end{tabular}

\subsection{Monte-carlo simulation of neutron scattering}

Monte Carlo simulations were used to study neutron scattering in the JLab calibration room. This helped to confirm the measurements we performed using Bonner spheres. A model of the calibration room at JLab was developed using Geant4. Steel walls with a thickness of 0.025 inches, 6 -inch thick concrete floor, $6 \mathrm{~m}$ thick soil and 0.031 - 
Chapter 5. Evaluation of neutron scattering effects in a JLab radiation-detector calibration facility using Bonner spheres

inch thick steel roof were included in the model. The CMU walls shown in Figure 5.8 were also included in the model. The detector platform, steel conveyor and the PVC pipe as illustrated in Figure 5.9 and Figure 5.10 were also included. Figure 5.14 shows the Geant4 model of the JLab calibration room that was developed in this study.

The energy deposition in ${ }^{3} \mathrm{He}$ proportional counters within the Bonner spheres were also modeled. Only events that deposited an energy of $190 \mathrm{keV}$ or above were considered as true neutron events (counts). An isotropic point neutron source based on IAEA standard Am/Be neutron spectrum was implemented in the simulation. The IAEA standard neutron spectrum is illustrated in Figure 5.15. The standard Geant4 physics list "QGSP_BERT_ HP" was included in the simulation with the addition of thermal neutron scattering cross sections. We discussed more details about the "QGSP_BERT_HP" physics list in Chapter 3 section 3.1.

We simulated the neutron count rates in Bonner spheres as a function of distance to the radiation source. Here, for brevity, we present data only for the $5^{\prime \prime}$ diameter sphere and the $12^{\prime \prime}$ diameter sphere. Figure 5.16 shows the comparison between the measured and the simulated count rates in the $12^{\prime \prime}$ diameter sphere multiplied by the square of the distance, as a function of distance. The errors given in the plot are statistical errors from measurements and simulations. As we discussed before, the direct neutron contribution from the source to the count rate follows a $1 / r^{2}$ behavior. But, as the scattered contribution is not so constrained, the scattered contribution can get larger as the source-to-detector distance increases. But, these scattered neutrons mainly lie in the thermal neutron region which makes it difficult for them to penetrate the thick polyethylene moderator of the $12^{\prime \prime}$ diameter sphere. Therefore, at larger distances statistical uncertainty can be large, causing the simulated result to deviate further away from the measurements. The data were fit to the linear model shown in equation 5.6. We leave off the quadratic term (indicated by parameter $\mathrm{R}$ in equation 
Chapter 5. Evaluation of neutron scattering effects in a JLab radiation-detector calibration facility using Bonner spheres

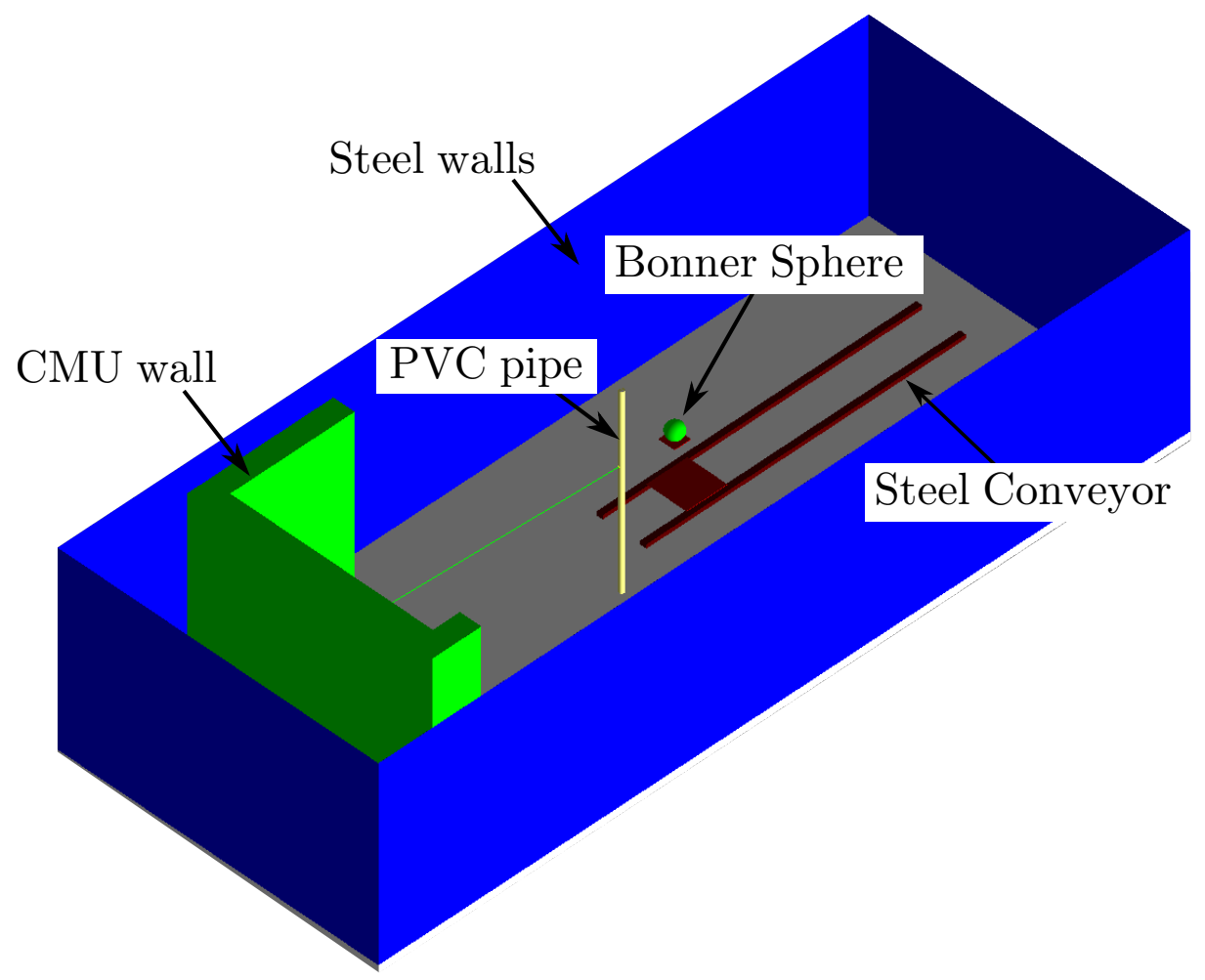

Figure 5.14: The Geant4 model of the JLab detector calibration facility. Steel walls of the room, A concrete wall, concrete floor, A PVC pipe, steel conveyor and a Bonner sphere is visible in the illustration. The roof and the $6 \mathrm{~m}$ thick soil beneath the floor of the facility is removed for illustration purposes. 
Chapter 5. Evaluation of neutron scattering effects in a JLab radiation-detector calibration facility using Bonner spheres

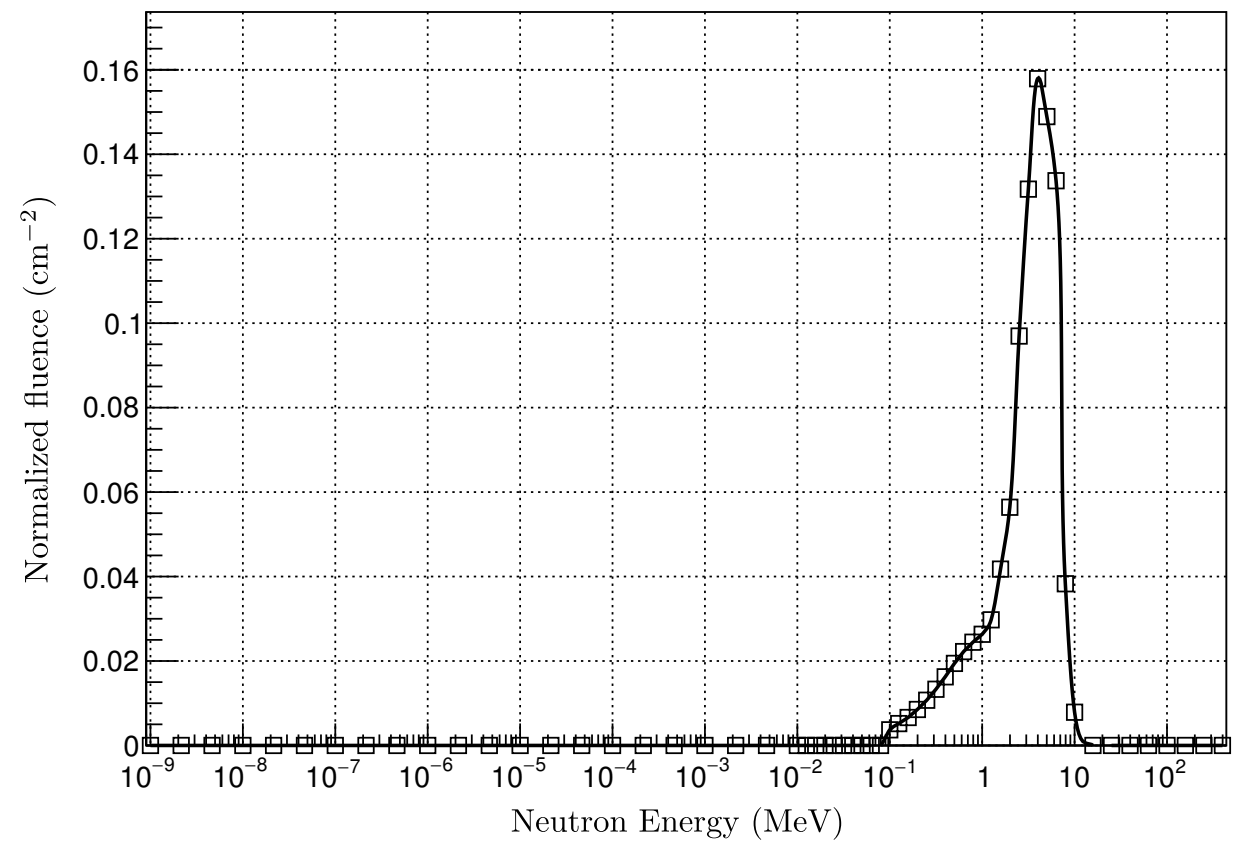

Figure 5.15: IAEA Am-Be neutron spectrum. The spectrum is constructed using 60 energy points and it is normalized. 
Chapter 5. Evaluation of neutron scattering effects in a JLab radiation-detector calibration facility using Bonner spheres

5.5) for the reasons discussed earlier. The scattered neutron contribution, from both the room and air, to the count rate roughly follows a $1 / d$ behavior.

Figure 5.17 shows a similar comparison of the $5^{\prime \prime}$ diameter Bonner sphere. The statistical uncertainty is smaller compared to the $12^{\prime \prime}$ Bonner sphere as more scattered neutrons can enter the $5^{\prime \prime}$ diameter sphere. Air moisture in the calibration room can affect the neutron scattering. Thermal neutrons can get easily scattered from hydrogen atoms in water. This effect can cause an increase in the count rates in the detectors and it will be more noticeable in smaller spheres. In Geant4 simulations we did not take the moisture level in the calibration room into account and this might explain the larger disagreement in $5^{\prime \prime}$ Bonner sphere compared to the $12^{\prime \prime}$ Bonner sphere.

A straight forward method to separate the scattering contributions using Monte Carlo simulation is to simulate the count rates in Bonner spheres with and without certain elements in the Geant4 model of the calibration room. We calculated the count rates in $12^{\prime \prime}$ and $5^{\prime \prime}$ diameter Bonner spheres similar to the method we described earlier. At the beginning, the model of the calibration room (including walls, floor, ceiling and all the support structures) was removed from the simulation except the Bonner sphere and the neutron source. The surrounding of the Bonner sphere was filled with low density gas (the effective implementation of vacuum in Geant4) and simulated the count rate in Bonner spheres as a function of source-to-detector distance. Then we simulated the count rates again, after including the model of the calibration room in the simulation. Finally we replaced the low density gas with dry air.

Figure 5.18 and Figure 5.19 show the count rate multiplied by the square of the distance as a function of the distance plots for the $12^{\prime \prime}$ and $5^{\prime \prime}$ diameter spheres respectively. In both these plots, the horizontal lines (black triangles) represent mea- 
Chapter 5. Evaluation of neutron scattering effects in a JLab radiation-detector calibration facility using Bonner spheres

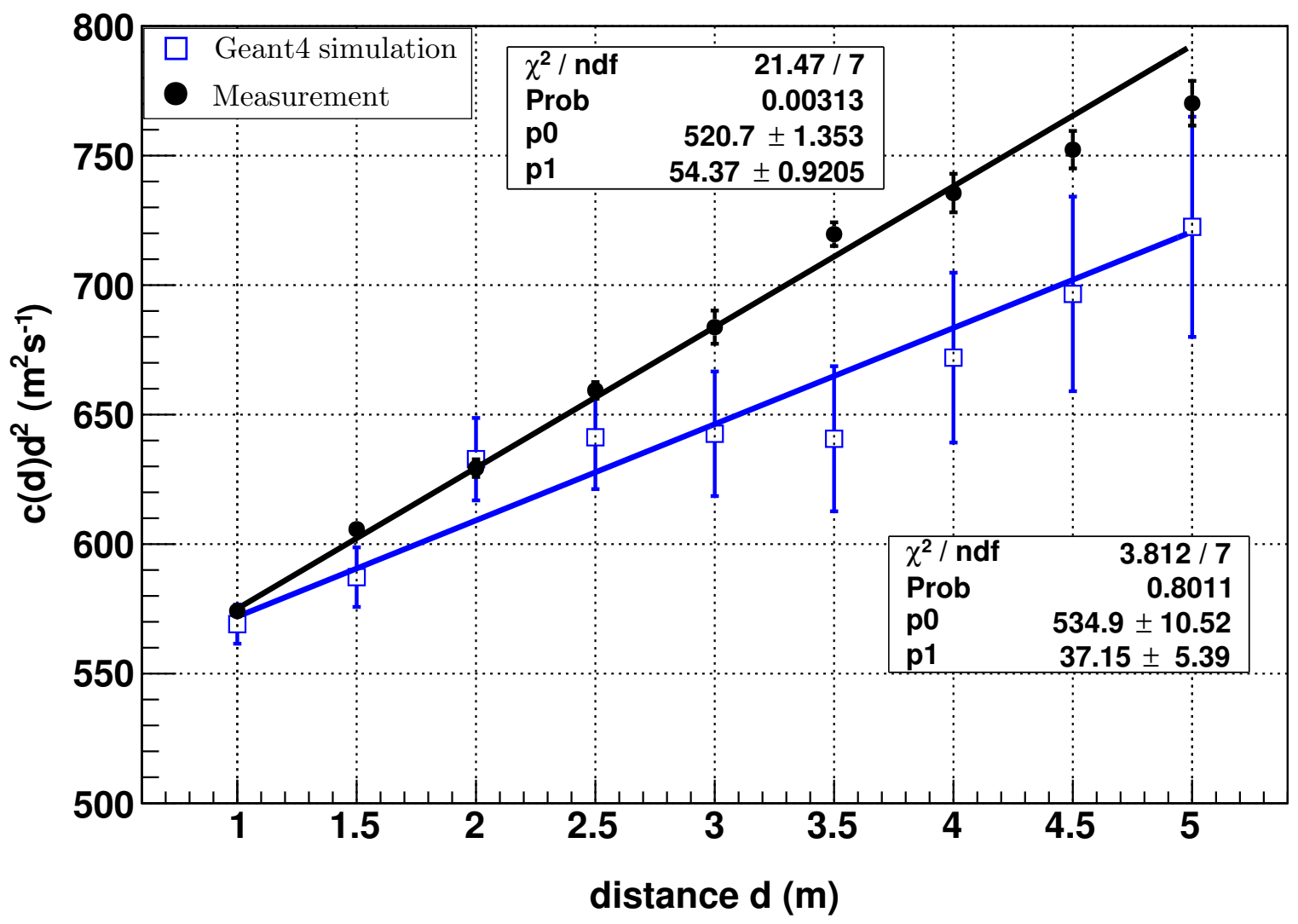

Figure 5.16: Measured and the simulated count rates in the $12^{\prime \prime}$ diameter Bonner sphere. The $y$-axis is the count rate multiplied by the square of the distance. The $\mathrm{x}$-axis is the source-to-detector distance. The errors on the blue points are statistical errors from the simulation. The data were fit to a linear equation. 
Chapter 5. Evaluation of neutron scattering effects in a JLab radiation-detector calibration facility using Bonner spheres

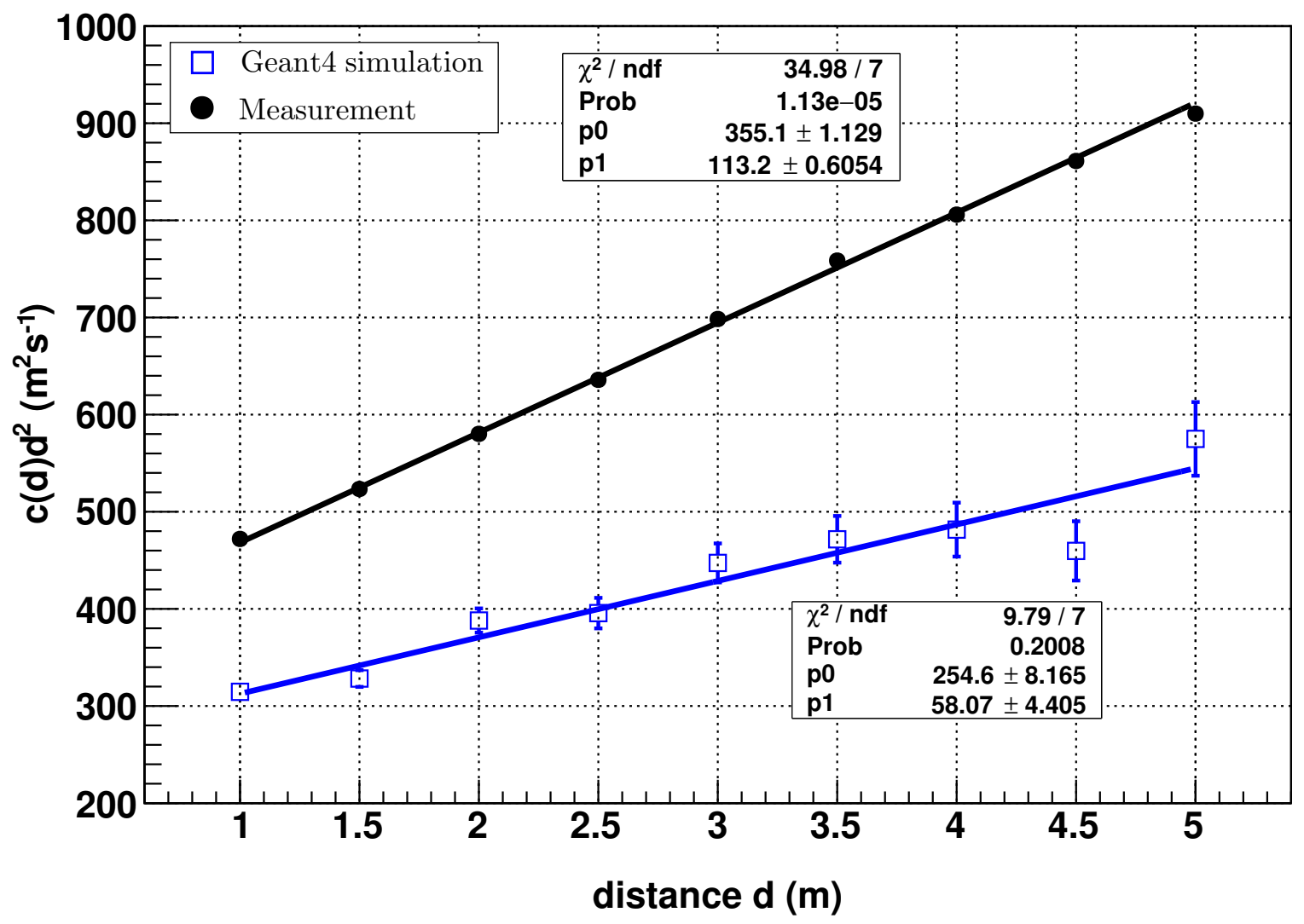

Figure 5.17: Measured and the simulated count rates in the $5^{\prime \prime}$ diameter Bonner sphere. The $y$-axis is the count rate multiplied by the square of the distance. The $\mathrm{x}$-axis is the source-to-detector distance. The errors on the blue points are statistical errors from the simulation. The data were fit to a linear equation. 
Chapter 5. Evaluation of neutron scattering effects in a JLab radiation-detector calibration facility using Bonner spheres

surements when there is no room and the surrounding has low density gas. In this situation there is no scattering contribution and the count rates in detectors follow $1 / d^{2}$. As can be seen in both Figure 5.18 and Figure 5.19, when the room was added into the model (red circles), it increases the scattering and there is only a slight increase due to the addition of dry air (blue squares) into the model. This confirms our hypothesis that the room scattering in the JLab calibration facility follows a $1 / d$ behavior and it is dominated by the effects other than the air.

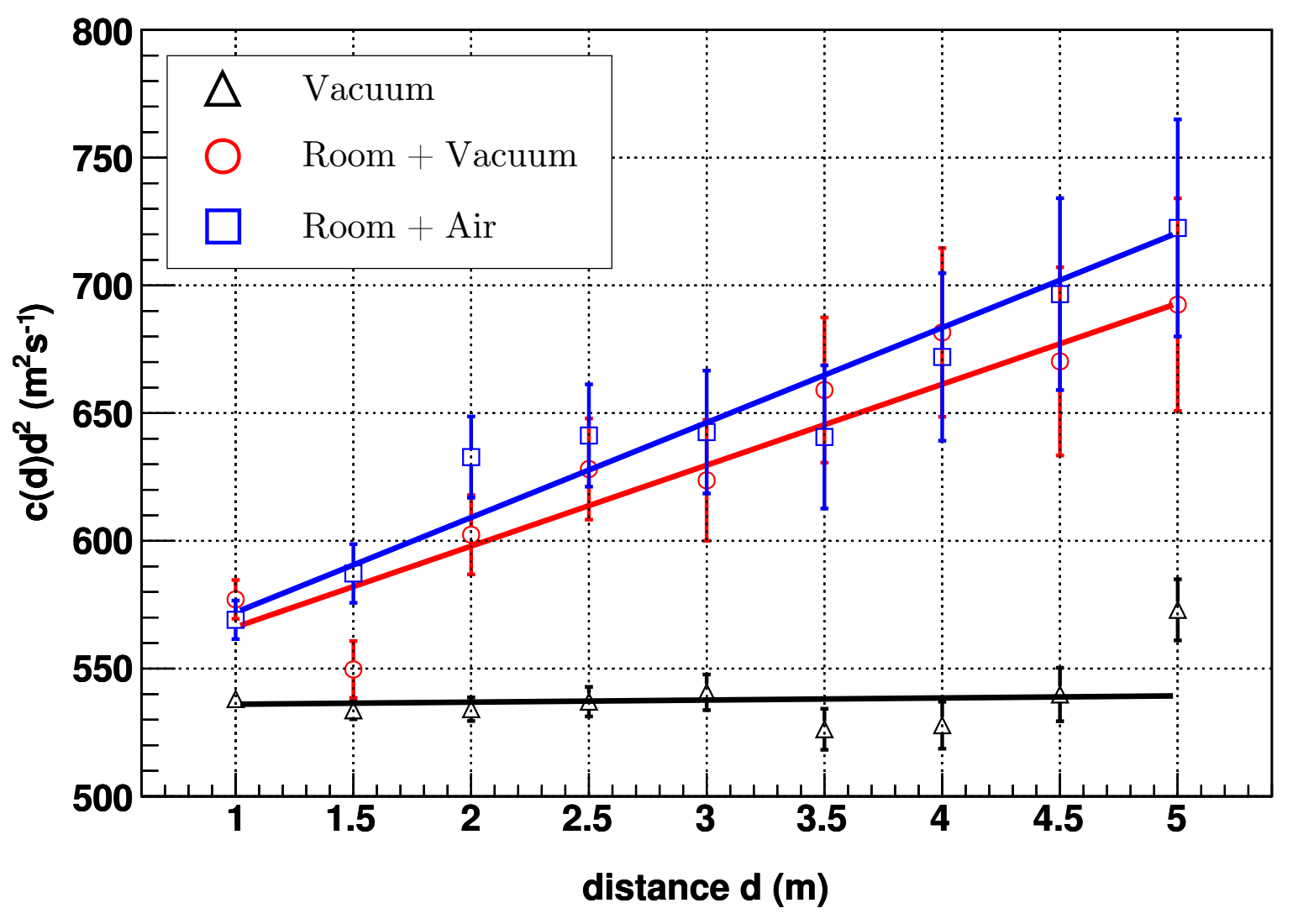

Figure 5.18: Calculation of count rates in the $12^{\prime \prime}$ diameter sphere in different environments. The y-axis of the plot is count rate multiplied by the square of distance and the $\mathrm{x}$-axis is the source-to-detector distance. The black triangles represent calculated values when the Bonner sphere is in low density gas (Vacuum). The red circles represent values when the calibration room was included and the blue squares indicate values when dry air was added. 
Chapter 5. Evaluation of neutron scattering effects in a JLab radiation-detector calibration facility using Bonner spheres

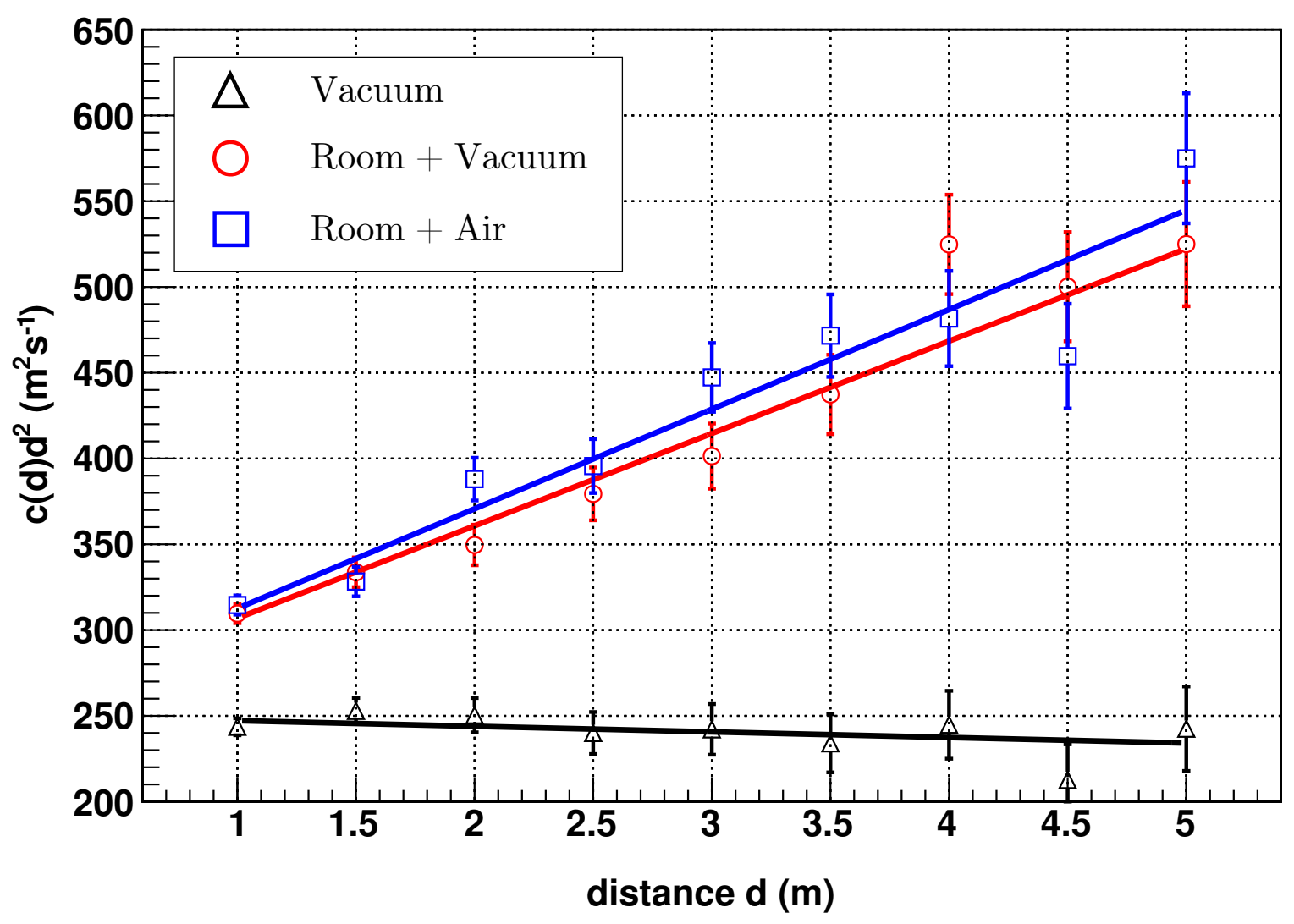

Figure 5.19: Calculation of count rates in the $5^{\prime \prime}$ diameter sphere in different environments. The y-axis of the plot is count rate multiplied by the square of distance and the $\mathrm{x}$-axis is the source-to-detector distance. The black triangles represent calculated values when the Bonner sphere is in low density gas (Vacuum). The red circles represent values when the calibration room was included and the blue squares indicate values when dry air was added. 
Chapter 5. Evaluation of neutron scattering effects in a JLab radiation-detector calibration facility using Bonner spheres

\subsection{Analyzing scattered neutron spectra}

Typically Bonner spheres are used to measure neutron counts which in turn are used to reconstruct the unknown neutron spectrum. One of the end goals of this project was also to reconstruct neutron spectra in the calibration room. The un-scattered neutron counts we calculated in the previous section ideally corresponds to a scatterfree neutron spectrum. We can also consider the neutron spectrum at $1 \mathrm{~m}$ from the radiation source as the scatter free neutron spectrum, as at this distance the scattering contribution is minimal. However, at larger distances from the radiation source, the relative contribution from scattered neutrons are larger than the direct neutrons, which will be reflected in the reconstructed neutron spectra. To isolate the scattered neutron spectra, a spectral subtraction method can be used.

The first step towards neutron spectral reconstruction is to calculate response functions of Bonner spheres. There are many calculations of Bonner sphere responses in literature [83] [94] [95]. But, it is always a good idea to repeat the calculations as a consistency check.

A Geant4 Monte Carlo code was developed to calculate the response functions of all 5 Bonner spheres and the bare detector. Each Bonner sphere was irradiated separately with neutrons using 60 discrete energies. The total energy deposited in the

${ }^{3}$ He for each event was calculated and saved as a pulse height spectrum. Figure 5.20 shows two pulse height spectra from the $12^{\prime \prime}$ diameter Bonner sphere. A low energy cut-off of $190 \mathrm{keV}$ was used in the selection of neutron events (counts). Figure 5.21 shows the calculated response functions for all the Bonner spheres. In the figure, "PE" stands for polyethylene and the number following the letters indicates the diameter. PE9L1 is the polyethylene sphere with a lead layer in it. The response of the bare detector was scaled by 0.7 to plot it in the same figure. 
Chapter 5. Evaluation of neutron scattering effects in a JLab radiation-detector calibration facility using Bonner spheres

Unfolding neutron spectra from Bonner sphere measurements involves finding a solution to the Fredholm equation given in equation 5.1. Many computer codes have been developed to solve this equation. These include BUNKI [96], which uses an iterative recursion method, MAXED [99], an algorthm based on maximum entropy principle, FRUIT [100], an unfolding code that runs within LabView, SWIFT [97], a Monte Carlo program and BONDI-97 [98], an algorithm based on artificial intelligence. In this work, we decided to use an iterative algorithm known as Maximum Likelihood Expectation Maximization (MLEM) to reconstruct the neutron spectra. Our initial attempts to unfold neutron spectra in JLab calibration facility are included in Appendix B. More work need to be done in terms of reconstruction algorithm, before studying the scattered neutron spectra. The calculated Bonner sphere response functions, shown in Figure 5.21, can be used for neutron spectral reconstructions.
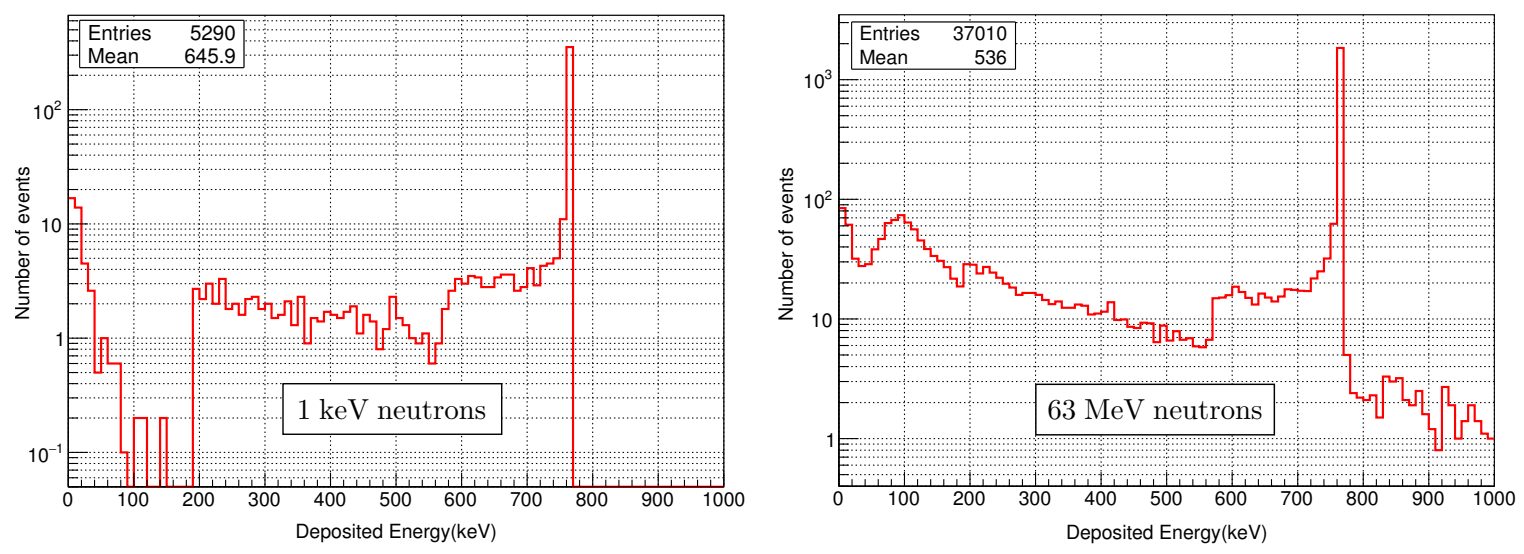

Figure 5.20: Pulse height spectra generated from the simulation of 12-inch Bonner sphere with $1 \mathrm{keV}$ neutrons (left) and $63 \mathrm{MeV}$ neutrons (right) from a point source located $1 \mathrm{~m}$ away from the detector. 
Chapter 5. Evaluation of neutron scattering effects in a JLab radiation-detector calibration facility using Bonner spheres

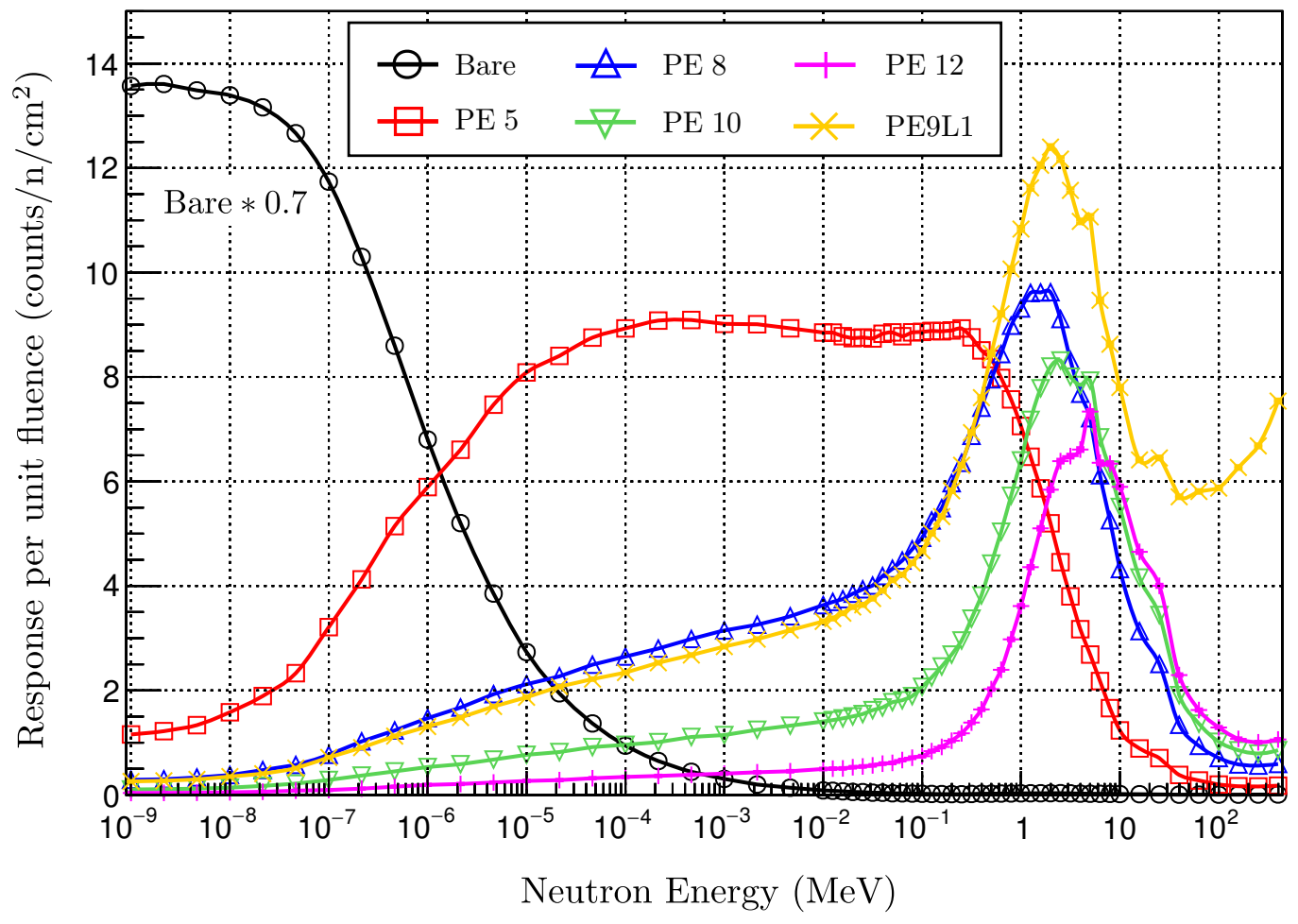

Figure 5.21: Calculated response functions using Geant4 simulation package. Response of the bare detector was scaled by 0.7 . 
Chapter 5. Evaluation of neutron scattering effects in a JLab radiation-detector calibration facility using Bonner spheres

\subsection{Summary of evaluating neutron scattering ef- fects using Bonner spheres}

Bonner-sphere project started with the intention of studying and characterizing neutron scattering at the calibration room at JLab. That goal was achieved with success. As a part of this study, we predicted the count rate that should register in a Bonner sphere, when it is in a neutron field of an Am/Be source. When we compared calculated count rates with measured count rates, we got an excellent agreement. The agreement between our own controlled measurement and the simulation result indicate the level of confidence we have in our simulations. This also an example of a successful Geant4 application in thermal neutron studies.

Apart from simulating the scatter-free count rates, Geant4 simulations were used to study the neutron scattering in the calibration room and we were able to predict the scattered count rates within $40 \%$ of the measurement. The simulation results indicate that the room scattering is larger than the neutron scattering in air. The neutron scattering can further be studied using spectral subtraction methods. The calculated response functions of Bonner spheres will be used for neutron spectral reconstruction in future work. More work needs to be done in terms of reconstructing the neutron spectra. With our results on neutron scattering in the calibration facility at JLab, RadCon will be able to perform more accurate detector calibrations. 


\section{Chapter 6}

\section{Summary}

High luminosity experiments that run at Jefferson Laboratory (JLab), pose a threat to the semiconductor electronics in the experimental halls due to radiation. The $A^{\prime}$ (A-prime) experiment, also known as APEX, is a high luminosity experiment that is approved to run in JLab Hall A. In the APEX experiment, a high energy electron beam $(1-4 \mathrm{GeV})$ will be incident on a tungsten target of thickness $2.8 \% X_{0}-5.3 \% X_{0}$, where $X_{0}$ is the radiation length. The pairs of $e^{+} e^{-}$leaving the target at a central angle of $5^{\circ}$ will be detected by the Hall A High Resolution Spectrometers (HRS). The equipment failures at the location of the HRS electronics due to radiation during the ${ }^{208} \mathrm{~Pb}$ radius experiment (PREX-I), which ran in JLab Hall A in 2010, pushed JLab authorities to enforce much strict radiation safety measures before approving experiments.

The main focus of this thesis was to address the radiation-related questions that needed to be answered in the APEX radiation review. The Geant4 Monte Carlo simulation tool was used to develop a detailed model of the JLab Hall A. We simulated several past experiments, including PREX-I, as well as the proposed APEX experiment. We calculated the radiation environments at different locations in the 
JLab Hall A for all these experiments. The damage to silicon electronics due to different types of radiation can be efficiently expressed in terms of the fluence of $1 \mathrm{MeV}$ neutrons. A relative comparison of radiation levels in Hall A was carried out, using Geant4 simulations, for experiments including APEX and PREX-I. We found that the radiation-damage fluence at the HRS electronics for the APEX experiment will be a factor of $\sim 9$ lower than that in PREX-I. We further demonstrated the possibility of lowering the radiation at HRS electronics by another factor of $\sim 3$, by installing a tungsten collimator in the beam pipe. This radiation study was a critical part of the APEX experiment readiness review and the subsequent approval of the beam time for the APEX experiment.

The simulations that provided a relative comparison of the radiation that was experienced during PREX-I and that is expected during APEX would mean nothing if we did not have confidence in our results. This thesis describes multiple studies that provide such confidence, and we summarize them here:

- In section 3.1, we describe Geant4 simulations of simple geometries that were also studied by the RadCon group using Geant3 in which excellent agreement was achieved.

- In section 3.1 we also describe a direct comparison of our simulation of PREX-I with simulations performed by the PREX-I collaboration, taking care to keep the inputs very similar, in which again excellent agreement at the 20-30\% level was achieved.

- In section 3.4.1 we describe a comparison of our simulation with published studies of radiation-damage in SiPM's that provided a well-calibrated measurement of the radiation during PREX-I. The studies of Ref. [63] were $\sim 30 \%$ below our simulations. 
- In section 3.4.2 we describe absolute comparisons of our simulations of with measurements performed during PREX-I and RCS. While naively our simulations were considerably higher than the numbers recorded by neutron probes, agreement came within a factor of two when the effects of realistic response curves and dead time were taken into account. To be certain of this absolute comparison, further studies of dead-time effects need to be performed.

- In section 5.3.3, in studies aimed at understanding issues that are important when calibrating neutron detectors, we performed our own well-controlled measurements with Bonner spheres and showed agreement with simulations at better than the $10 \%$ level. This measurements will be discussed more below.

In addition to radiation studies, we described the characterization of a Scintillating Fiber (SciFi) detector that will play a critical role in the energy resolution of APEX. The APEX experiment will look for a resonance peak on top of a background which mainly consists of $e^{+} e^{-}$pairs from radiative and Bethe-Heitler processes. To find a narrow peak on top of a continuous background, we need a very good mass resolution. Therefore, the sensitivity to $A^{\prime}$ depends mainly on precise reconstruction of the invariant mass of $e^{+} e^{-}$pairs. In the $A^{\prime}$ experiment, the mass resolution is dominated by the track measurement uncertainties. The typical calibration procedure of the HRS spectrometer, the sieve slit method, will not provide the required mass resolution in the right HRS (positron arm). Therefore, a novel scintillating fiber (SciFi) detector has been constructed to measure the position of a charged particle at the target plane. Using a radiation source, we tested the efficiency of one of the two SciFi detectors which will be used in the APEX experiment. Analyzing pulse height spectra of the SciFi/PMT, we calculated that there are $\sim 6$ photoelectrons generated in the PMT for each event in the SciFi. Based on the calculated efficiency of the 
SciFi detector, we estimated the event rate expected during the APEX calibration run of the HRS.

As described in Chapter 5, we performed a series of measurements that quantified scattering effects that are important when calibrating neutron detectors using a calibrated source. Specifically, it is crucial to understand neutron scattering from walls and air in a calibration room. We characterized neutron scattering in the JLab calibration room using measurements from Bonner spheres and compared with simulations. The work improves the understanding that the RadCon has of calibrations of neutron detectors. The work also provided our most precise comparison of direct calibrated neutron-detector measurements with Geant4 simulations and we found better than $10 \%$ agreement.

The work presented in this thesis contributed to reach the goals of the APEX experiment. The SciFi detectors that will be used in both arms of the HRS will improve the track reconstruction of the $e^{+} e^{-}$pairs, giving rise to a good mass resolution. We are confident that the radiation in JLab Hall A during the APEX experiment will not damage the HRS electronics. Based on our studies on neutron scattering in JLab calibration room, neutron detectors will have more accurate calibrations. With the techniques we have established, we believe not only APEX, but also other future high luminosity experiments will be benefited immensely. 


\section{Bibliography}

[1] Brun, R., et al. "GEANT3 user guide, CERN data handling division DD." EE/841 (1985). (Cited on p. 2)

[2] Agostinelli, Sea, et al. "GEANT4a simulation toolkit." Nuclear instruments and methods in physics research section A: Accelerators, Spectrometers, Detectors and Associated Equipment 506.3 (2003): 250-303. (Cited on p. 2, 37, 39)

[3] Ferrari, Alfredo, et al. FLUKA: A multi-particle transport code (Program version 2005). No. INFN-TC-05-11. 2005. (Cited on p. 2, 82)

[4] Gaillard, Mary K., Paul D. Grannis, and Frank J. Sciulli. "The standard model of particle physics." Reviews of Modern Physics 71.2 (1999): S96.

[5] Spergel, David N., et al. "First-year Wilkinson Microwave Anisotropy Probe (WMAP)* observations: determination of cosmological parameters." The Astrophysical Journal Supplement Series 148.1 (2003): 175. (Cited on p. 3)

[6] Essig, Rouven, et al. "Dark sectors and new, light, weakly-coupled particles." arXiv preprint arXiv:1311.0029 (2013). (Cited on p. xiii, 4, 6, 7, 8)

[7] Bjorken, J. D., et al. "The A Experiment (APEX): Search for a New Vector Boson A Decaying to e+e." (Cited on p. 5, 6, 8, 26)

[8] B. Holdom, Phys. Lett. B 166 (1986) 196. (Cited on p. 5) 
[9] Hook, Anson, Eder Izaguirre, and Jay G. Wacker. "Model-Independent Bounds on Kinetic Mixing." Advances in High Energy Physics 2011 (2011). (Cited on p. 5)

[10] P. Galison and A. Manohar, Phys. Lett. B 136 (1984) 279. (Cited on p. 5)

[11] Bjorken, James D., et al. "New fixed-target experiments to search for dark gauge forces.” Physical Review D 80.7 (2009): 075018. (Cited on p. 6, 8, 9, 11)

[12] J. D. Bjorken et al., Phys. Rev. D 38 (1988) 3375. (Cited on p. 6)

[13] E. M. Riordan et al., Phys. Rev. Lett. 59 (1987) 755. (Cited on p. 6)

[14] A. Bross, M. Crisler, S. H. Pordes, J. Volk, S. Errede and J. Wrbanek, Phys. Rev. Lett. 67 (1991) 2942. (Cited on p. 6)

[15] M. Davier and H. Nguyen Ngoc, Phys. Lett. B 229, 150 (1989). (Cited on p. 6)

[16] R. Essig. A' motivation \& status, APEX's role. In APEX collaboration meeting 2015, JLab, 2015. (Cited on p. xiii, 7)

[17] The Heavy Photon Search Collaboration (HPS), https://confluence.slac. stanford.edu/display/hpsg/ (Cited on p. 6)

[18] Balewski, Jan, et al. "DarkLight: a search for dark forces at the Jefferson laboratory free-electron laser facility." arXiv preprint arXiv:1307.4432 (2013). (Cited on p. 6)

[19] Merkel, Harald, et al. "Searches for dark photons at the Mainz Microtron." AIP Conference Proceedings. Vol. 1563. No. 1. AIP, 2013. (Cited on p. 6)

[20] B. Aubert et al. [BABAR Collaboration], Phys. Rev. Lett. 103, 081803 (2009) [arXiv:0905.4539 [hep-ex]]. (Cited on p. 6, 8)

[21] KLOE-2 collaboration. "Search for dark Higgsstrahlung in $e+e-\rightarrow \mu+\mu-$ and missing energy events with the KLOE experiment." Physics Letters B 747 (2015): 365-372. (Cited on p. 8) 
[22] Alcorn, John, et al. "Basic instrumentation for hall a at jefferson lab." Nuclear Instruments and Methods in Physics Research Section A: Accelerators, Spectrometers, Detectors and Associated Equipment 522.3 (2004): 294-346. (Cited on p. xv, 13, 14, $22,25,32,47)$

[23] Adriani, Oscar, et al. "An anomalous positron abundance in cosmic rays with energies 1.5100 GeV." Nature 458.7238 (2009): 607-609. (Cited on p. 4)

[24] Ackermann, Markus, et al. "Fermi LAT observations of cosmic-ray electrons from 7 GeV to 1 TeV." Physical Review D 82.9 (2010): 092004. (Cited on p. 4)

[25] Chang, J., et al. "An excess of cosmic ray electrons at energies of $300800 \mathrm{GeV}$." Nature 456.7220 (2008): 362-365. (Cited on p. 4)

[26] Aharonian, F., et al. "Energy spectrum of cosmic-ray electrons at TeV energies." Physical Review Letters 101.26 (2008): 261104. (Cited on p. 4)

[27] C. Amsler et al. (Particle Data Group), Phys. Lett. B 667, 1 (2008). (Cited on p. 10)

[28] Finkbeiner, Douglas P., et al. "Consistent scenarios for cosmic-ray excesses from Sommerfeld-enhanced dark matter annihilation." Journal of Cosmology and Astroparticle Physics 2011.05 (2011): 002. (Cited on p. 4)

[29] Bennett, Gerald W., et al. "Final report of the E821 muon anomalous magnetic moment measurement at BNL." Physical Review D 73.7 (2006): 072003. (Cited on p. 5)

[30] Pospelov, Maxim. "Secluded U(1) below the weak scale." Physical Review D 80.9 (2009): 095002. (Cited on p. 5)

[31] Abrahamyan, S., et al. "Search for a new gauge boson in electron-nucleus fixed-target scattering by the APEX experiment." Physical Review Letters 107.19 (2011): 191804. (Cited on p. xiii, xiv, xxiv, 9, 12, 13, 34, 36, 117, 121) 
[32] Abrahamyan, Sergey, et al. "Measurement of the neutron radius of $\mathrm{Pb} 208$ through parity violation in electron scattering." Physical review letters 108.11 (2012): 112502. (Cited on p. 15)

[33] Horowitz, Charles J., Krishna S. Kumar, and R. Michaels. "Electroweak measurements of neutron densities in CREX and PREX at JLab, USA." arXiv preprint arXiv:1307.3572 (2013). (Cited on p. 15)

[34] Silviu Covrig Dusa. "The APEX Target." APEX Experiment Readiness Review, 07 April 2016, Jefferson Lab, Newport News, VA. Presentation. (Cited on p. 20)

[35] E. Jensen, "HRS Trigger and PID" at "Searching for a New Gauge Boson at JLab" (Sept 2010), https://www.jlab.org/conferences/boson2010/Tuesday/ HRS\%20Fast $\% 20$ Trigger\%20and\%20PID\%20Capability.pdf (Cited on p. 34)

[36] Riordan, Seamus, et al. "Comparison between Wiser rates calculation and data from transversity and PVDIS experiments." Internal document (2014). (Cited on p. 26)

[37] D. Wiser Ph.D. thesis, University of Wisconsin (1977). (Cited on p. 26)

[38] OConnell, J., and J. Lightbody. "Computer codes QFS and EPC." Comp. in Phys (1998). (Cited on p. 26)

[39] Degtyarenko, P. V., M. V. Kossov, and H-P. Wellisch. "Chiral invariant phase space event generator." The European Physical Journal A-Hadrons and Nuclei 9.3 (2000): 411-420. (Cited on p. 26)

[40] Prex-ii: Precision parity-violating measurement of the neutron skin of lead. Hall a collaboration proposal, Jefferson Lab PAC 38, 2011. (Cited on p. 15, 37, 53)

[41] Abrahamyan, Sergey, et al. "Measurement of the neutron radius of $\mathrm{Pb} 208$ through parity violation in electron scattering." Physical review letters 108.11 (2012): 112502. (Cited on p. 53) 
[42] Exclusive compton scattering on the proton. Hall a collaboration proposal, Jefferson Lab, 1999. (Cited on p. 57)

[43] Fissum, K. G., et al. "Vertical drift chambers for the Hall A high-resolution spectrometers at Jefferson Lab." Nuclear Instruments and Methods in Physics Research Section A: Accelerators, Spectrometers, Detectors and Associated Equipment 474.2 (2001): 108-131. (Cited on p. 32)

[44] International Commission on Radiation Units and Measurements (1985). Determination of dose equivalents resulting from external radiation sources, Part 1, Report 39 (Cited on p. 79)

[45] International Commission on Radiation Units and Measurements (1980). Radiation quantities and units. Report 33

[46] Calibration of Radiation Protection Monitoring Instruments Safety Reports Series No. 16 (2000)

[47] Sannikov, A. V., and E. N. Savitskaya." Ambient dose equivalent conversion factors for high energy neutrons based on the ICRP 60 recommendations." Radiation protection dosimetry 70.1-4 (1997): 383-386.

[48] Andersson, I. ., and Josef Braun. A neutron rem counter. No. AE-132. Aktiebolaget Atomenergi, Stockholm (Sweden), 1964. (Cited on p. 81)

[49] International Atomic Energy Agency, Vienna (Austria) (2001). Compendium of neutron spectra and detector responses for radiation protection purposes Supplement to technical reports series no 318. International Atomic Energy Agency (IAEA): IAEA. (Cited on p. 79, 174)

[50] Tessler, G., and S. S. Glickstein. "Monte Carlo calculation of the response of the 
portable neutron monitor SNOOPY." Health physics 28.3 (1975): 197-204. (Cited on p. $86,204,208)$

[51] Mares, V., A. V. Sannikov, and H. Schraube. "Response functions of the AnderssonBraun and extended range rem counters for neutron energies from thermal to $10 \mathrm{GeV} . "$ Nuclear Instruments and Methods in Physics Research Section A: Accelerators, Spectrometers, Detectors and Associated Equipment 476.1 (2002): 341-346. (Cited on p. $86,204)$

[52] Srour, J. R., Cheryl J. Marshall, and Paul W. Marshall. "Review of displacement damage effects in silicon devices." IEEE Transactions on Nuclear Science 50.3 (2003): 653-670. (Cited on p. 61, 62)

[53] Lindstrm, Gunnar. "Radiation damage in silicon detectors." Nuclear Instruments and Methods in Physics Research Section A: Accelerators, Spectrometers, Detectors and Associated Equipment 512.1 (2003): 30-43. (Cited on p. 61)

[54] Messenger, S. R., et al. "Nonionizing energy loss (NIEL) for heavy ions." IEEE Transactions on Nuclear Science 46.6 (1999): 1595-1602. (Cited on p. 62)

[55] Dale, C. J., et al. " A comparison of Monte Carlo and analytic treatments of displacement damage in Si microvolumes." IEEE transactions on nuclear science 41.6 (1994): 1974-1983. (Cited on p. 62)

[56] Huhtinen, M., and P. A. Aarnio. "Pion induced displacement damage in silicon devices." Nuclear Instruments and Methods in Physics Research Section A: Accelerators, Spectrometers, Detectors and Associated Equipment 335.3 (1993): 580-582. (Cited on p. 62 )

[57] Summers, Geoffrey P., et al. "Damage correlations in semiconductors exposed to gamma, electron and proton radiations." IEEE Transactions on Nuclear Science 40.6 (1993): 1372-1379. (Cited on p. 62, 68) 
[58] Akkerman, A., et al. "Updated NIEL calculations for estimating the damage induced by particles and $\gamma$-rays in Si and GaAs." Radiation Physics and Chemistry 62.4 (2001): 301-310. (Cited on p. 62, 68)

[59] Van Ginneken, A. Nonionizing energy deposition in silicon for radiation damage studies. No. FERMILAB-FN-0522. 1989. (Cited on p. 62)

[60] Vasilescu, A., and G. Lindstroem. "Displacement damage in silicon, on-line compilation." http://rd50.web.cern.ch/RD50/NIEL/default.html (2000). (Cited on p. $63)$

[61] Vasilescu, Angela, and Gunnar Lindstrm. "Notes on the fluence normalisation based on the NIEL scaling hypothesis." ROSE/TN 2000 (2000). (Cited on p. 63)

[62] Standard practice for characterizing neutron energy fluence spectra in terms of an equivalent monoenergetic neutron fluence for radiation-hardness testing of electronics. E 722 04, ASTM, 2005. (Cited on p. 63)

[63] Qiang, Yi, et al. "Radiation hardness tests of SiPMs for the JLab Hall D Barrel calorimeter." Nuclear Instruments and Methods in Physics Research Section A: Accelerators, Spectrometers, Detectors and Associated Equipment 698 (2013): 234-241. (Cited on p. 76, 77, 189)

[64] https://halldweb.jlab.org/wiki/index.php/SiPM_Radiation_Hardness_Test (Cited on p. 77)

[65] M. Brugger for the FLUKA Team. Estimating damage 2 electronics using fluka, July 2008. (Cited on p. 63)

[66] https://www.jlab.org/Hall-B/run-web/hps/ESAD_HallB-HPS.pdf (Cited on p. $63)$ 
[67] Knoll, Glenn F. Radiation detection and measurement. John Wiley and Sons, 2010. (Cited on p. 88)

[68] Moyers, M. F., et al. "Leakage and scatter radiation from a double scattering based proton beamline." Medical physics 35.1 (2008): 128-144. (Cited on p. 88)

[69] Reed, Robert, et al. "Test Report of Proton and Neutron Exposures of Devices that utilize Optical Components and are contained in the CIRS Instrument." Greenbelt,(Maryland) NASA/Goddard Space Flight Center 20771 (1997). (Cited on p. 63)

[70] Radiation Control Department - JLab, HPP-CAL-017, Calibration of probes for use with Radiation Boundary Monitors (RBMs). (2013) (Cited on p. 90)

[71] Jensen, Eric. A Search for a New Gauge Boson A. Diss. The College of William and Mary, 2012. (Cited on p. xxiv, 29, 36, 119, 121)

[72] Neil Goeckner-Wald and Bogdan Wojtsekhowski "Technical Note - Calibration of High Resolution Spectrometer Optics for APEX Experiment, 2011.” (Cited on p. $122,148)$

[73] Matt Kriete and Bogdan Wojtsekhowski "Technical Note - A Scintillating Fiber Detector for Magnetic Optics Calibration of the $A^{\prime}$ Experiment, 2012." (Cited on p. $122,123)$

[74] Brooks, F. D., and H. Klein. "Neutron spectrometryhistorical review and present status." Nuclear Instruments and Methods in Physics Research Section A: Accelerators, Spectrometers, Detectors and Associated Equipment 476.1 (2002): 1-11. (Cited on p. xi, 154, 155)

[75] Bramblett, Richard L., Ronald I. Ewing, and T. W. Bonner. " A new type of neutron 
spectrometer." Nuclear Instruments and Methods 9.1 (1960): 1-12. (Cited on p. xxvii, $154,157)$

[76] Matzke, Manfred. "Unfolding procedures." Radiation Protection Dosimetry 107.1-3 (2003): 149-168. (Cited on p. 158)

[77] Matzke, Manfred. "Propagation of uncertainties in unfolding procedures." Nuclear Instruments and Methods in Physics Research Section A: Accelerators, Spectrometers, Detectors and Associated Equipment 476.1 (2002): 230-241. (Cited on p. 158)

[78] Crane, T. W., and M. P. Baker. "Chapter 13,Neutron Detectors,." Passive Nondestructive Assay of Nuclear Materials, edited by TD Reilly, N. Ensslin, and HA Smith, US Nuclear Regulatory Commission NUREG/CR-5550 (1991). (Cited on p. xxvii, $158,159,160)$

[79] Wiegel, B., and A. V. Alevra. "NEMUSthe PTB neutron multisphere spectrometer: Bonner spheres and more." Nuclear Instruments and Methods in Physics Research Section A: Accelerators, Spectrometers, Detectors and Associated Equipment 476.1 (2002): 36-41. (Cited on p. 158)

[80] Mares, V., and H. Schraube. "High energy neutron spectrometry with Bonner spheres." (1997). (Cited on p. 158)

[81] Birattari, C., et al. "The extended range neutron rem counter LINUS: overview and latest developments." Radiation Protection Dosimetry 76.3 (1998): 135-148. (Cited on p. xxi, 82, 87, 158, 208)

[82] Dubeau, Jacques, et al. "A new type of neutron spectrometer using nested moderator." Electronic Proceedings of the International Workshop on Real-Time Measurement Instrumentation and Control (RTMIC), IEEE-NPSS. 2010. (Cited on p. 161) 
[83] Garny, S., V. Mares, and W. Rhm. "Response functions of a Bonner sphere spectrometer calculated with GEANT4." Nuclear Instruments and Methods in Physics Research Section A: Accelerators, Spectrometers, Detectors and Associated Equipment 604.3 (2009): 612-617. (Cited on p. xxviii, 161, 184)

[84] Hunt, J. B. "The calibration of neutron sensitive spherical devices." Radiation protection dosimetry 8.4 (1984): 239-251. (Cited on p. 164, 169)

[85] Bedogni, Roberto, and Francisco Fernndez Moreno. Neutron spectrometry and dosimetry for radiation protection around a high energy electron/positron collider. Universitat Autnoma de Barcelona,, 2006. (Cited on p. 174)

[86] Shepp, Lawrence A., and Yehuda Vardi. "Maximum likelihood reconstruction for emission tomography." IEEE transactions on medical imaging 1.2 (1982): 113-122. (Cited on p. 210)

[87] Internal JLab communication" by P. Degtiarenko (Cited on p. 164)

[88] Papov, Valdimir "Technical note-3He neutron counters setup for Bonner Sphere neutron Spectrometer." Internal JLab communication" (Cited on p. 163)

[89] Rakitha Sanjeewa Beminiwattha. personal communication. (Cited on p. xvi, 43)

[90] Bertini, Hugo W., and Miriam P. Guthrie. "News item results from medium-energy intranuclear-cascade calculation." Nuclear Physics A 169.3 (1971): 670-672. (Cited on p. 39)

[91] Geant4 Physics Reference Manual. http://geant4.web.cern.ch/ geant4/UserDocumentation/UsersGuides/PhysicsReferenceManual/fo/ PhysicsReferenceManual.pdf (Cited on p. 39)

[92] Patrignani, C., and Particle Data Group. "Review of particle physics." Chinese physics C 40.10 (2016): 100001. (Cited on p. 42) 
[93] Bellamy, E. H., et al. "Absolute calibration and monitoring of a spectrometric channel using a photomultiplier." Nuclear Instruments and Methods in Physics Research Section A: Accelerators, Spectrometers, Detectors and Associated Equipment 339.3 (1994): 468-476. (Cited on p. 133)

[94] Alevra, Alexandru V. "Neutron spectrometry with Bonner spheres: applications in physics and dosimetry." Fifth International Conference on Applications of Nuclear Techniques: Neutrons in Research and Industry. International Society for Optics and Photonics, 1997. (Cited on p. 184)

[95] Vylet, V. "Response matrix of an extended Bonner sphere system." Nuclear Instruments and Methods in Physics Research Section A: Accelerators, Spectrometers, Detectors and Associated Equipment 476.1 (2002): 26-30. (Cited on p. 184)

[96] Lowry, Kimberly A., and Tommy L. Johnson. "Modifications to recursion unfolding algorithms to find more appropriate neutron spectra." Health physics 47.4 (1984): 587-593. (Cited on p. 185)

[97] Sanna, R., and K. O'brien. "Monte-Carlo unfolding of neutron spectra." Nuclear Instruments and Methods 91.4 (1971): 573-576. (Cited on p. 185)

[98] Mukherjee, Bhaskar. "BONDI-97: a novel neutron energy spectrum unfolding tool using a genetic algorithm." Nuclear Instruments and Methods in Physics Research Section A: Accelerators, Spectrometers, Detectors and Associated Equipment 432.2 (1999): 305-312. (Cited on p. 185)

[99] Reginatto, Marcel, and Paul Goldhagen. "MAXED, a computer code for maximum entropy deconvolution of multisphere neutron spectrometer data." Health Physics 77.5 (1999): 579-583. (Cited on p. 185)

[100] Bedogni, Roberto, et al. "FRUIT: an operational tool for multisphere neutron spectrometry in workplaces." Nuclear Instruments and Methods in Physics Research 
Section A: Accelerators, Spectrometers, Detectors and Associated Equipment 580.3 (2007): 1301-1309. (Cited on p. 185) 


\section{Appendix A}

\section{Geant4 simulations of the response function of the neutron detector SNOOPY}

The NP100B neutron detector, also known as SNOOPY, is a polyethylene cylinder with a thermal neutron detector at the center of the cylinder. Thermal neutron detector is filled with $\sim 0.8 \mathrm{~atm} \mathrm{BF}_{3}$ gas. The SNOOPY has several layers of moderating material. Figure A.1 illustrates the measured dimensions of a typical SNOOPY detector that is used at JLab. These dimensions are very similar to the dimensions used in SNOOPY simulations performed by Tessler et. al [50] and also Mares et. al. [51].

Figure A.2 illustrates a cross section of the SNOOPY detector and Figure A.3 illustrates an exploded view. Figure A.4, Figure A.5 and Figure A.6 illustrate three attenuators made from boron doped rubber. All the images are taken from the Geant 4 simulation developed in this study. The composition of materials were taken from the Ref. [51].

The physics list used was the standard "QGSP_BERT_HP" hadronic model with 
Appendix A. Geant4 simulations of the response function of the neutron detector SNOOPY

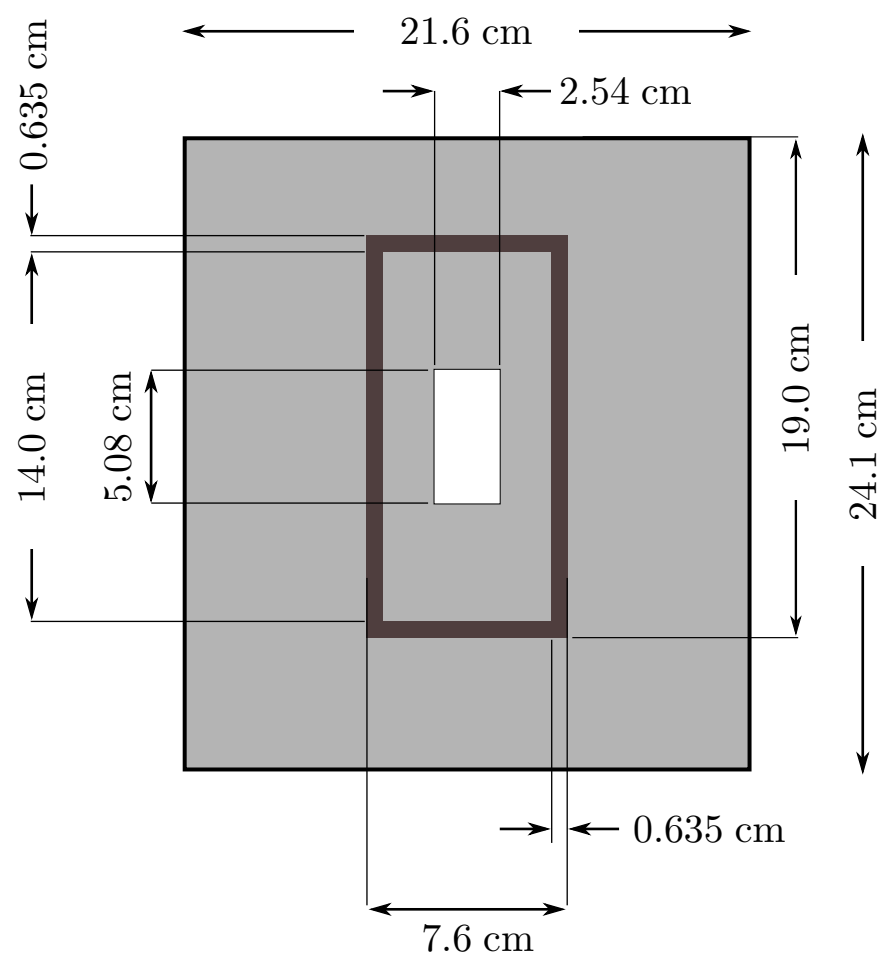

$\square \quad \mathrm{BF}_{3}$ proportional counter

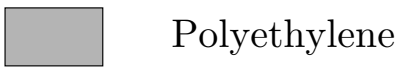

Boron doped rubber

Figure A.1: The dimensions and the components of the NP100B (SNOOPY) neutron detector. 
Appendix A. Geant4 simulations of the response function of the neutron detector SNOOPY

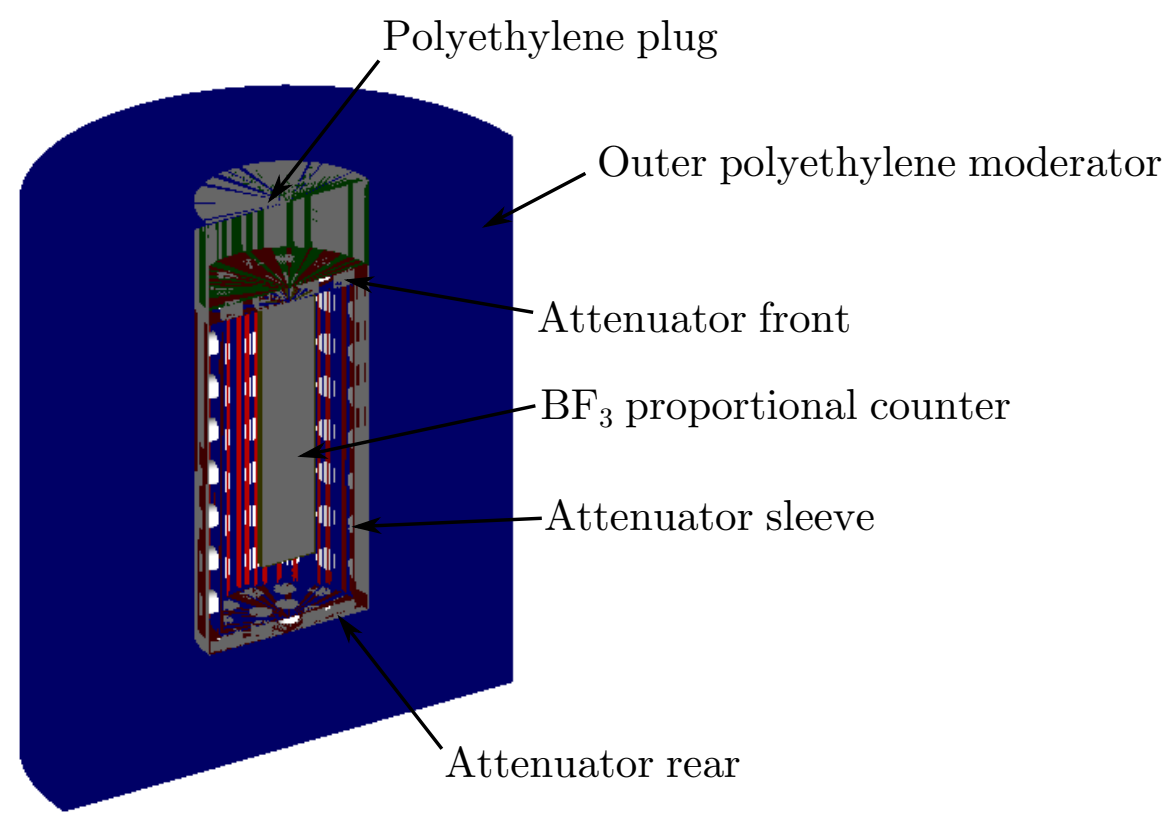

Figure A.2: A cross section view of the SNOOPY detector. The image is taken from the Geant4 model we developed.

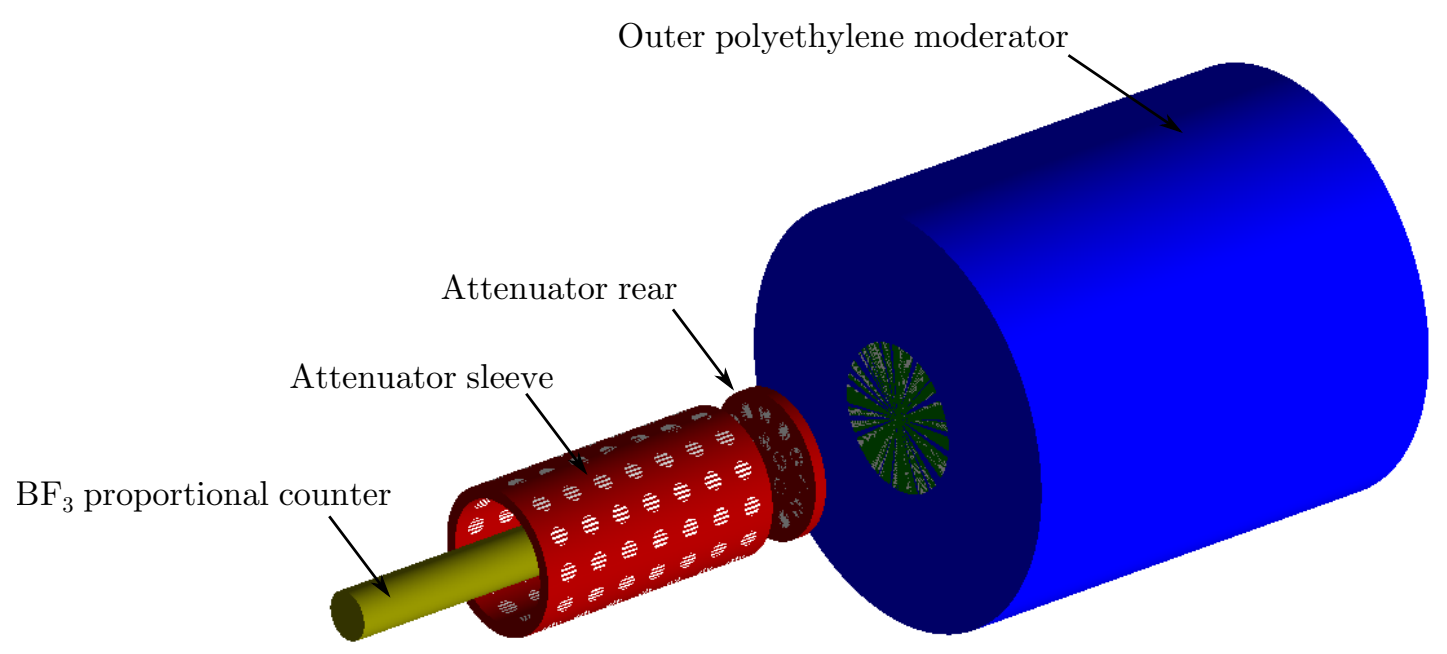

Figure A.3: An exploded view of the SNOOPY. The front attenuator is not shown in the picture. 
Appendix A. Geant4 simulations of the response function of the neutron detector

$0.95 \mathrm{~cm}\left(3 / 8^{\prime \prime}\right)$ diamter 12 holes on a $5.4 \mathrm{~cm}\left(21 / 8^{\prime \prime}\right)$ diamter circle

$0.95 \mathrm{~cm}\left(3 / 8^{\prime \prime}\right)$ diamter 6 holes on a $2.54 \mathrm{~cm}\left(1^{\prime \prime}\right)$ diamter circle

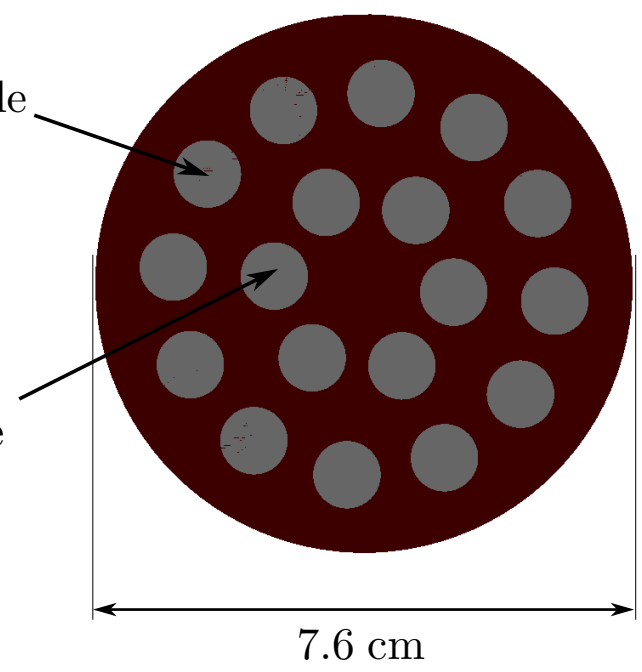

Figure A.4: A detailed view of the rear attenuator of the SNOOPY detector. The thickness of the cicular shaped attenuator is $0.635 \mathrm{~cm}$. The holes with diameter of $0.95 \mathrm{~cm}$ are through holes.

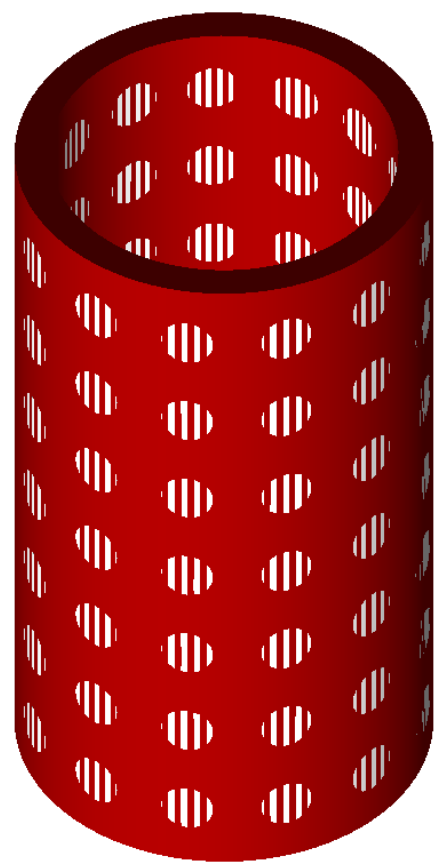

$0.95 \mathrm{~cm}\left(3 / 8^{\prime \prime}\right)$ diameter 91 holes

13 equally spaced rows.

The typical distance between

two holes is $1.9 \mathrm{~cm}\left(3 / 4^{\prime \prime}\right)$.

Figure A.5: A detailed view of the attenuator sleeve. Wall thickness of the sleeve is $0.635 \mathrm{~cm}$. The holes with diameter of $0.95 \mathrm{~cm}$ are through holes. 
Appendix A. Geant4 simulations of the response function of the neutron detector SNOOPY

$0.95 \mathrm{~cm}\left(3 / 8^{\prime \prime}\right)$ diamter 12 holes on a $5.4 \mathrm{~cm}\left(21 / 8^{\prime \prime}\right)$ diamter circle

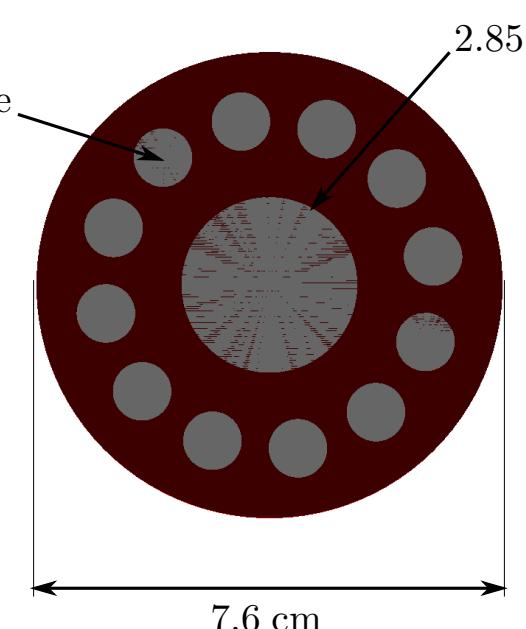

Figure A.6: A detailed view of the attenuator at the front of the detector. The thickness of the cicular shaped attenuator is $0.635 \mathrm{~cm}$. The holes with diameter of $0.95 \mathrm{~cm}$ and the large center hole with a diameter of $2.85 \mathrm{~cm}$ are through holes.

thermal scattering models. To simulate the SNOOPY detector response, mono energetic neutrons were incident laterally on the entire area of the detector. Neutrons with 60 different energies ranging from $10^{-9} \mathrm{MeV}$ to $400 \mathrm{MeV}$ were used for the simulation. About $1 \times 10^{7}$ histories were used for each neutron energy. Energy deposition in $\mathrm{BF}_{3}$ gas was recorded and only events with deposited energy larger than $0.48 \mathrm{MeV}$ was considered as true neutron events (counts). Figure A.7 shows the calculated response function using the Geant4 simulation developed in this study. It agrees very well with the calculations done by Tessler and Glickstein [50]. We also compared our calculations with FLUKA calculations done by the Birattari and colleagues where we have a very good agreement [81]. Figure 3.35 illustrates the comparison and it agrees with several measurements as well. 
Appendix A. Geant4 simulations of the response function of the neutron detector SNOOPY

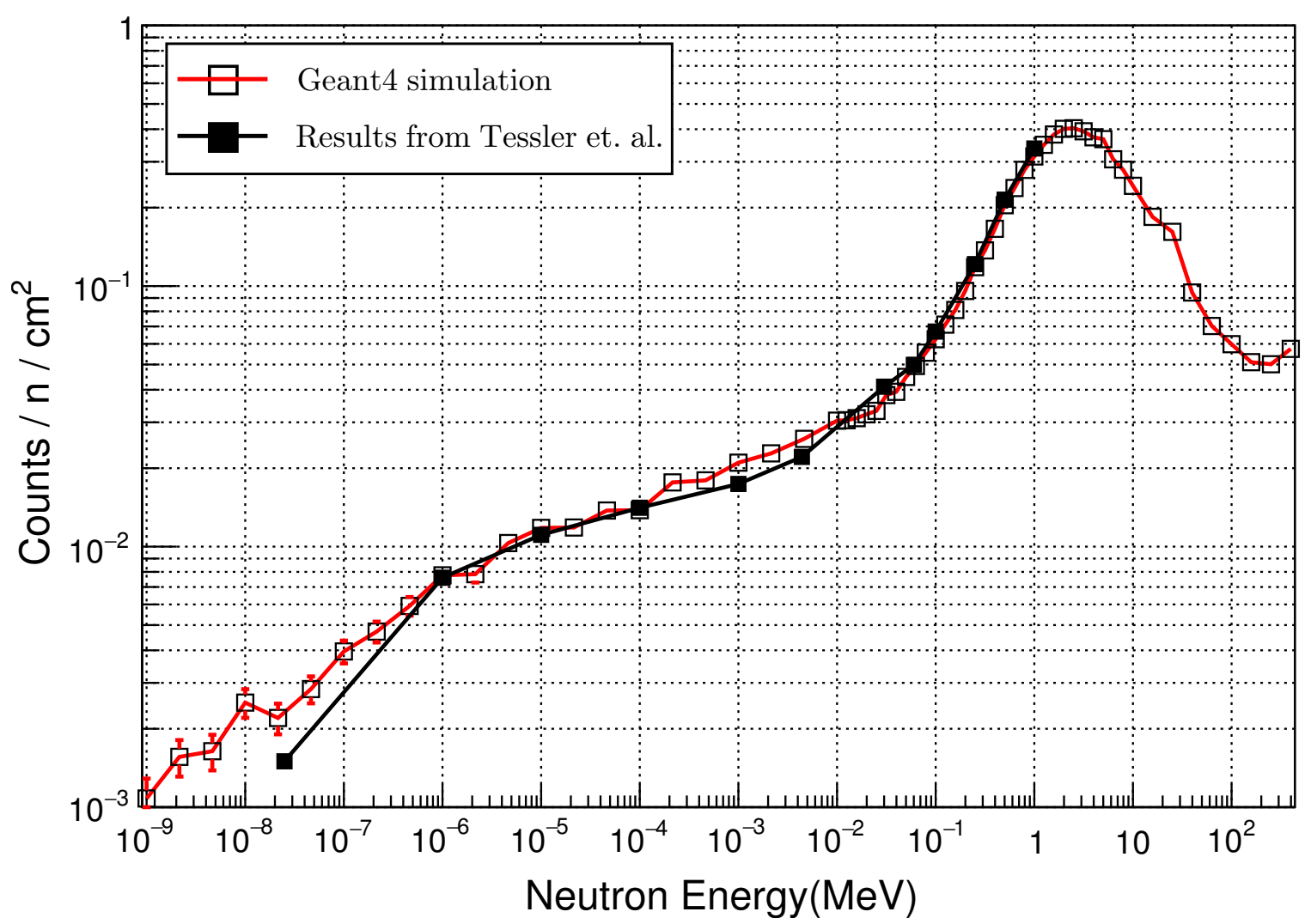

Figure A.7: The response function of the SNOOPY calculated using Geant4 simulations is compared with the similar calculations by Tessler et. al. The y-xis of the plot is the counts per unit fluence and the $\mathrm{x}$-axis is the neutron energy in $\mathrm{MeV}$. 


\section{Appendix B}

\section{Maximum-likelihood}

\section{expectation-maximization}

\section{(MLEM) unfolding}

The Bonner sphere spectrometers (BSS) are commonly used to determine an unknown neutron spectra in accelerator facilities. In this section I describe an attempt to unfold neutron spectrum of an Am/Be source based on measurements from 5 bonner spheres.

To unfold the measured data, we used the Maximum-likelyhood expectationmaximization (MLEM) algorithm in which 5 measurements (excluding bare detector) were deconvolved into a 60-bin neutron energy spectrum. MLEM is a standard statistical reconstruction algorithm that is used in positron emission tomography (PET) [86]. The MLEM algorithm maximizes the likelihood of obtaining the measured data $m$ from a spectrum $\phi$ as follows. 


$$
x_{j}^{k+1}=\frac{x_{j}^{k}}{\sum_{i=1}^{N} R_{i j}} \sum_{i=1}^{N} R_{i j} \frac{m_{i}}{\sum_{j=1}^{J} R_{i j} x_{j}^{k}}
$$

Here, $m_{i}$ is the measurement, $R_{i j}$ is the response function of the detector and $x_{j}^{k}$ is the spectrum estimate for $j^{\text {th }}$ energy bin in $k^{\text {th }}$ iteration. MLEM algorithm can be described in three steps:

(a) Start with an initial estimate for the spectrum $x^{0}$.

(b) If $x^{k}$ denotes the estimate of $x$ at the $\mathrm{k}^{\text {th }}$ iteration, calculate the new $x^{k+1}$ by equation B.1.

(c) If the resulting spectrum $x^{k+1}$ gives an acceptable result then stop, else return to step (b).

A stopping criterion was introduced to stop the algorithm when the sum of differences between the measured counts and the calculated counts based on the deconvoluted spectrum, $\sum_{i=1}^{N}\left|m_{i}-\sum_{j=1}^{J} R_{i j} x_{j}^{k}\right|$, reaches a minimum value.

Figures B.1 and Figure B.2 illustrate reconstructed Am-Be spectra starting from two different guess spectra. The measured count rates were multiplied by the calibration factor before applying them in the spectrum unfolding algorithm. Since we did not have measurements for an arbitrary location in the calibration hall, I used measurements at $1 \mathrm{~m}$ from the Am-Be source. In this case these $m_{i}$ values are equivalent to calculated count rates in Table 5.4. To reconstruct spectrum in Figure B.1, I used a constant value for all energy bins as the guess spectrum. It took 640097 iterations to complete this spectrum. On the other hand for the spectrum in Figure B.2, I used a guess spectrum with non-zero values only in energy bins between $0.1 \mathrm{MeV}$ and $10 \mathrm{MeV}$. It took 687973 iterations to complete. 


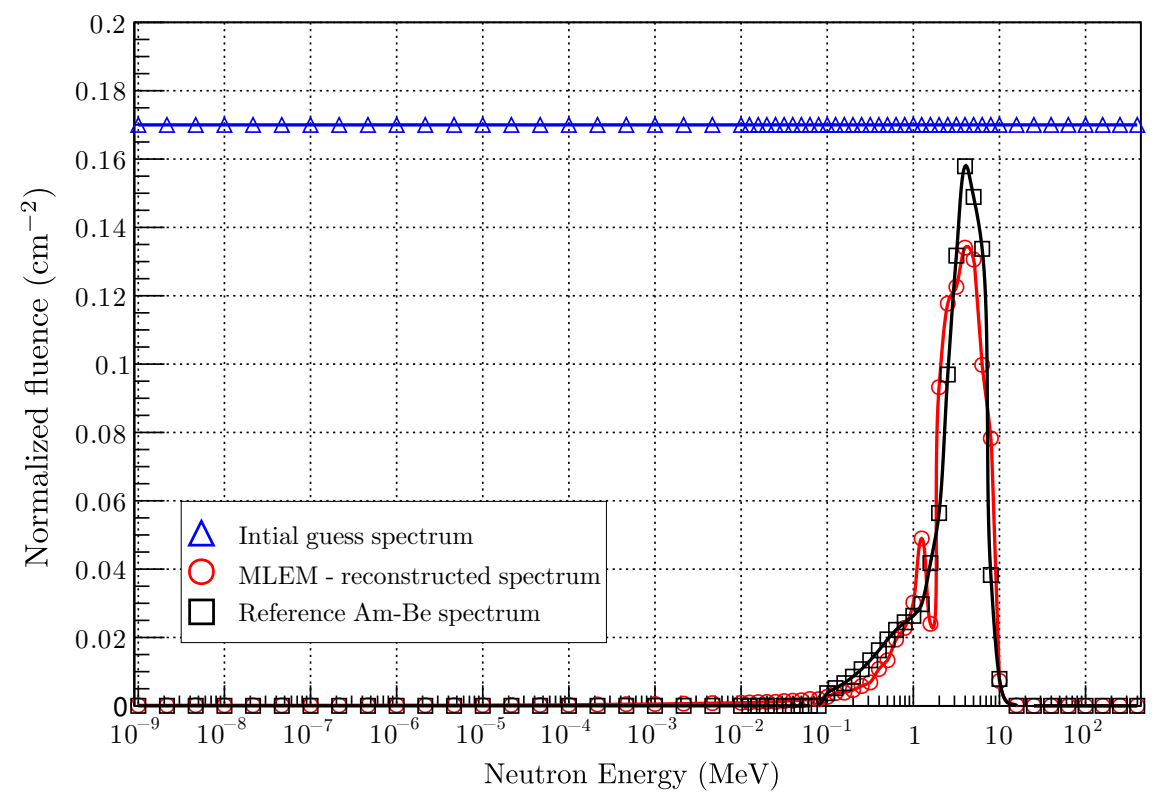

Figure B.1: Reconstructed Am-Be spectrum using MLEM algorithm. A constant value was chosen for all the energy bins as the initial guess spectrum. 640097 iterations were taken to reconstruct this spectrum. Reference Am-Be spectrum is also shown here. 


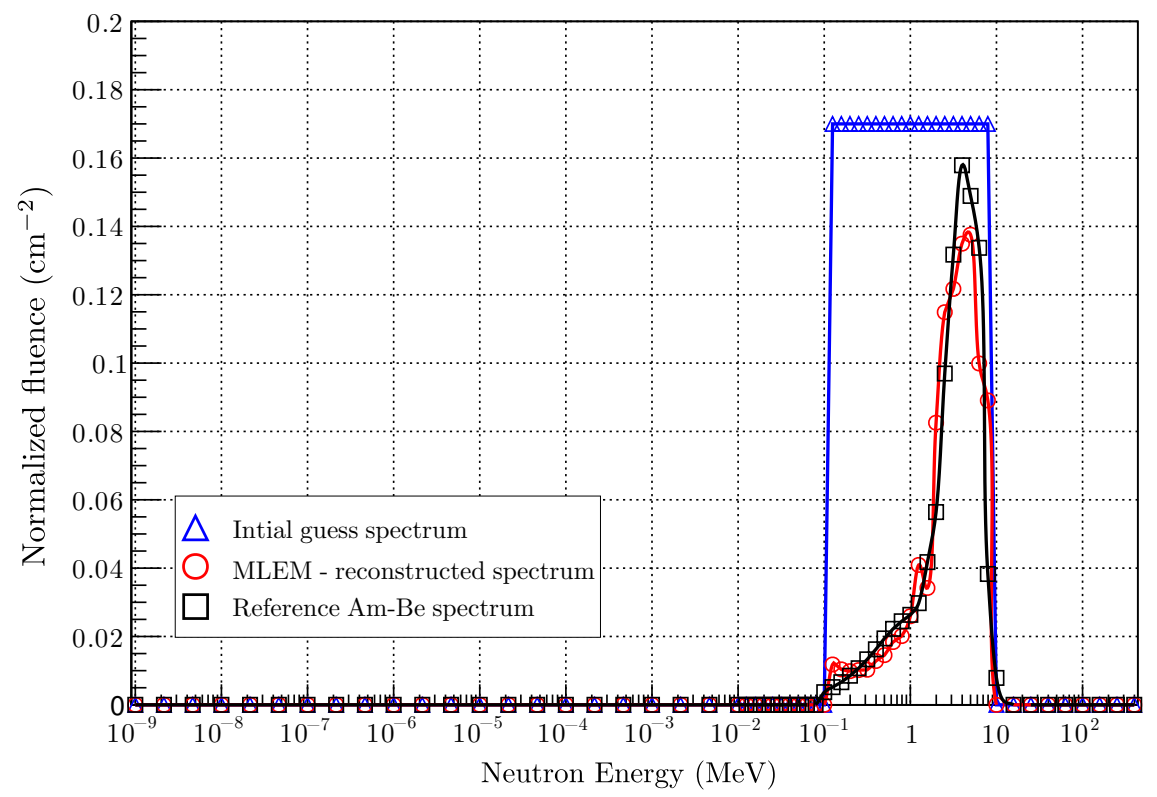

Figure B.2: Reconstructed Am-Be spectrum using MLEM algorithm. Guess spectrum bin values were set to zero except in the bins between $0.1 \mathrm{MeV}$ and $10 \mathrm{MeV}$. 687973 iterations were taken to reconstruct this spectrum. Reference Am-Be spectrum is also shown here. 